\title{
Abstracts of the 2018 AANS/CNS Joint Section on Disorders of the Spine and Peripheral Nerves Annual Meeting
}

\author{
Orlando, Florida • March 14-17, 2018 \\ (DOI: 10.3171/2018.3.FOC-ASPNabstracts)
}

\section{J.A.N.E. Award Presentation}

100 Lateral Lumbar Interbody Fusion in the Elderly: A 10 Year Experience

Nitin Agarwal MD; Andrew M Faramand MD; Nima Alan MD; Zachary J. Tempel MD; D. Kojo Hamilton MD; David O. Okonkwo MD, PhD; Adam S. Kanter MD

Introduction: Elderly patients, often presenting with multiple medical comorbidities, are touted to be at an increased risk of postoperative complications. As such, we describe our perioperative outcomes in this cohort of patients over the age of 70 following standalone LLIF.

Methods: A retrospective query of a prospectively maintained database was performed for patients over the age of 70 who underwent standalone LLIF. The preoperative and postoperative values for the Oswetry Disability Index (ODI) were analyzed to compare outcomes after intervention. Femoral neck t-scores of the study cohort were acquired from the bone density scans and correlated with the incidence of graft subsidence. Statistical analysis using t-test was performed with IBM SPSS Statistics (IBM, Armonk, NY).

Results: Among the study cohort of 54 patients, the median age at the time of surgery was 74 years (range, 70-87 years). Seventeen patients had at least 3 medical comorbidities at surgery. Twenty-two patients underwent a one level, and 32 patients had 2 or more level fusions. The median length of hospital stay was 2 days (range, 1-4 days). No statistically significant relationship was observed between the length of hospital stay and age at the time of surgery. There was one intraoperative death secondary to cardiac arrest, with a mortality rate of $1.9 \%$. One patient developed a transient femoral nerve injury. Five patients with radiographic graft subsidence subsequently required posterior instrumentation. A lower femoral neck t-score $<-1.0$ correlated with a higher chance of graft subsidence $(\mathrm{p}=0.006)$. The mean ODI 1 year postoperatively of 31.1 was significantly $(\mathrm{p}=0.003)$ less than the mean preoperative ODI of 46.2.

Conclusion: Standalone LLIF can be safely and effectively performed in the elderly population. Despite an association with increased comorbidities, age alone should not be a deterrent when considering an LLIF procedure in the elderly population.

\section{Mayfield Basic Science Award Presentation}

101 Engaging Cervical Spinal Cord Circuitry with Non-invasive Transcutaneous Electrical Stimulation and a Serotonin Agonist to Re-enable Volitional Control of Hand Function in Tetraplegic Patients

Nicholas Au Yong, MD, PhD; Yevgeniy Freyvert, MD; Tianyi Niu, MD; Erika Morikawa; Sharon Zudnowski; Melanie Sarino; Yury Gerasimenko; Reggie Edgerton, PhD; Daniel C. Lu, MD, PhD

Introduction: Spinal cord injury (SCI) affects significant societal and personal impact which scales with ascending level of injury. Recovery of upper extremity function is the top priority in the tetraplegic SCI population1. We investigate the combined effects of two neuromodulation strategies: transcutaneous electrical stimulation (TES) and buspirone pharmacological modulation, for promoting upper limb motor recovery in chronic cervical SCI tetraplegic subjects.

Methods: A double-blind study protocol was used to determine the effects of cervical electrical stimulation alone or in combination with the monoaminergic agonist buspirone on upper limb motor function in subjects with chronic motor complete (ASIA B) cervical injury $(n=6)$. Voluntary upper limb function was evaluated through measures of controlled hand contraction, handgrip force production, dexterity measures, and validated clinical assessment batteries. Subjects underwent pre-intervention assessment followed by three treatment phases with TES and buspirone or placebo. A delayed post-treatment testing period was used to assess for durable improvement in function.

Results: All subjects demonstrated improvement in hand strength and upper extremity functional metrics. Mean hand strength increased greater than $300 \%$ after transcutaneous electrical stimulation plus buspirone. A corresponding improvement was observed in upper extremity functional metrics. Functional improvements generally persisted after the study interventions were discontinued.

Conclusion: We demonstrate that, with these novel interventions, the cervical spinal circuitry can be neuromodulated to improve volitional control of hand function in tetraplegic subjects. The potential impact of these findings on individuals with upper limb paralysis could be dramatic functionally, psychologically, and economically.

\section{Mayfield Clinical Science Award Presentation}

102 Crossing the Cervicothoracic Junction in Posterior Cervical Decompression and Fusion: A Cohort Analysis

Kevin T. Huang, MD; Maya Harary, BA; Muhammad M AbdEl-Barr, MD, PhD; Yi Lu, MD, PhD; Michael W. Groff, MD, FACS; John H. Chi, MD, MPH

Introduction: Multilevel decompression and fusion is a wellknown procedure for spine surgeons. Current convention notes that the cervicothoracic junction (CTJ) has inherent instability and that surgeons should avoid ending constructs there. However, there exists little data to guide this practice. 1

Methods: We retrospectively reviewed a consecutive series of 130 adult patients who underwent multilevel posterior cervical decompression and fusion at our institution. All patients had surgery for degenerative cervical conditions and received a fusion that either stopped at C7 or extended beyond the CTJ. Data was collected on demographic, baseline, and intraoperative variables as well as any complications or subsequent needs for reoperation.

Results: A total of 67 patients had a fusion ending at the CTJ compared to 63 whose fusion crossed the CTJ. The groups were not significantly different in terms of age $(\mathrm{p}=0.4594)$, smoking status ( $\mathrm{p}=0.7753)$, American Society of Anesthesiologists score $(p=0.2856)$, or amount of available follow-up $(p=0.1437)$. Patients whose fusion crossed the CTJ had more levels fused (mean: $5.8 \pm$ 
2.0 vs. $3.5 \pm 0.8$ levels, $\mathrm{p}<0.0001$ ), longer surgical times (mean: $216.5 \pm 82.6$ vs. $148.5 \pm 44.7$ minutes, $\mathrm{p}<0.0001$ ), and higher $\mathrm{EBL}$ (mean: $480 \pm 775$ vs. $115 \pm 113 \mathrm{~mL}, \mathrm{p}=0.0002$ ). The groups did not differ significantly in overall reoperation rate (10.4\% vs. $9.5 \%$, $\mathrm{p}=1.0000$ ), but crossing the CTJ was associated with a higher rate of wound dehiscence $(7.9 \%$ vs. $0 \%, \mathrm{p}=0.0246)$. Those patients with constructs ending at the $\mathrm{CTJ}$ had higher rates of adjacent segment disease $(4.5 \%$ vs. $0 \%, \mathrm{p}=0.2449)$ and hardware complication $(4.5 \%$ vs. $1.6 \%, \mathrm{p}=0.6198)$, but these differences were not statistically significant.

Conclusion: The relative merits of crossing the CTJ in posterior cervical fusions are not a forgone conclusion. Deciding to cross the CTJ may involve balancing increased surgical times and wound complication rates on one hand with the possibility of increased adjacent segment disease and hardware complications on the other.

\section{Kline Peripheral Nerve Top Abstract Presentation}

103 Lipid-rich Vesicular Transport in Myelinating Schwann Cells In-vitro

\section{Joey Kevin Grochmal, MD, PhD}

Introduction: Peripheral nerve myelin synthesis requires a large volume of hydrocarbon molecules to support the exponential production of lipid bilayer inherent in its formation; this provision occurs through a process that is yet unclear.

Methods: Using spectral confocal microscopy, we studied exogenous BFP expressing Schwann cells as they progressively myelinated in GFP-DRG explant co-cultures (Figure 1: In-vitro myelination by exogenous BFP and endogenous GFP expressing Schwann cells, with myelin labelling by Nile Red. Exogenous Schwann cells produce myelin basic protein -inset). Noticing lipidrich Schwann cell domains by staining tissue living cultures with Nile Red, we then used time-lapse imaging to capture retrograde vesicular transport live in-vitro (Figure 2).

Results: Schwann cells are observed to transport lipid-rich vesicles with a spectral signature indicative of extensive cholesterol content, in the context of elongated alignment with DRG neurites (Figure 2- Lipid vesicles in bright yellow, Schwann cell cytoplasm in blue with evident nucleus). These vesicles move in a retrograde fashion (at a speed similar to that of retrograde axonal transport) and appear to coalesce in the perinuclear area, consistent with incorporation into the Golgi and rough endoplasmic reticulum organelles. They also experience sudden changes in velocity, suggesting that these are actively transported vesicles rather that passively diffusing lipid microdomains.

Conclusion: We provide initial evidence in support of active cytoplasmic transport by Schwann cells of lipid-rich vesicles, a phenomenon that may be a precursor to their use as building blocks in myelination.

\section{Charles Kuntz IV Scholars}

104 Early Kyphoplasty is Associated with Reduced Risk of Persistent Opioid Prescribing for Nearly 12,000 PrivatelyInsured Patients with Osteoporotic Vertebral Fractures

Jay Kiran Nathan, MD; Mitchell A Johnson, BS; Jennifer Waljee, MD; Nicholas J. Szerlip, MD, FACS; Paul Park, MD; Mark E. Oppenlander, MD

Introduction: Osteoporotic vertebral fracture is a painful condition typically requiring opioid analgesia. However, these particularly frail patients face increased risks with even short-term opioid use. There are mixed data on efficacy of kyphoplasty to reduce pain scores, and no large-scale data regarding opioid prescribing before versus after the procedure, the focus of the present study.

Methods: Using claims from a large private US insurer, we identified adults with osteoporotic vertebral fracture who underwent kyphoplasty from 2001 to 2014, had at least 1 year of continuous insurance enrollment around their index procedure, and were prescribed opioids during this time. Intensity of opioid prescribing was classified based on CDC guidelines and average daily oral morphine equivalents (OME), and duration of prescriptions relative to fracture and intervention was calculated.

Results: A total of 11,964 adults met inclusion criteria, 4418 $(36.9 \%)$ of whom were opioid naïve prior to fracture. Median age was 77 , and $71.9 \%$ were female. 10,365 patients $(86.6 \%)$ underwent only 1 kyphoplasty, which was single-level in $80.1 \%$ of cases. Intensity of daily OME prescribing before fracture remained the same or decreased for $55.9 \%$ of patients immediately after the procedure. $28.9 \%$ of patients were not prescribed any opioids beyond 30 days following kyphoplasty, while multivariate logistic regression identified younger age, female sex, pre-fracture opioid use, and delayed kyphoplasty as predictive of persistent use. Opioid naïve patients had a significantly shorter duration and lower intensity of opioid prescribing post-procedure.

Conclusion: Kyphoplasty can reduce the burden of opioid use among a frail population with osteoporotic vertebral fractures, but its efficacy depends on patient- and procedure-specific factors, most notably kyphoplasty timing and pre-fracture opioid exposure. This is in concordance with pain score data from recent randomized controlled trials, indicating greater improvement when kyphoplasty is performed early after fracture, rather than after a prolonged course of opioid therapy.

105 Natural History of the Aging Spine: A Cross Sectional Analysis of Radiographic Sagittal Alignment in Asymptomatic Patients

Mark Andrew Attiah, MD, MS, MBE; Yasmine Alkhalid, BS; Christine S Ahn, BS; Diane Villaroman; Bilwaj K Gaonkar, PhD; Tianyi Niu, MD; Joel S Beckett, MD; MHS; Luke Macyszyn, $\mathrm{MD}, \mathrm{MA}$

Introduction: Spinal alignment in the sagittal plane has become increasingly important. Surgical correction goals have been predominately from patients with spinal disease/deformity. We performed a retrospective, cross-sectional analysis in a healthy population to quantify the natural history of spinal alignment with age.

Methods: Sagittal, full-length radiographs of 211 asymptomatic patients were evaluated (age range 18 to 80 ). The following parameters and relationships were measured or calculated: Cervical lordosis (CL), thoracic kyphosis (TK), lumbar lordosis (LL), pelvic incidence (PI), truncal inclination (TI), sagittal vertical axis (SVA), cervical sagittal vertical axis (cSVA), and T1 slope, T1 slope/CL, TK/LL, and LL-PI. Patients were subdivided 
by age in decades. Regression analysis was performed to determine correlation between age and treatment.

Results: Cervical lordosis $(r=0.6)$, thoracic kyphosis $(r=$ $0.8)$, and truncal inclination $(r=0.36), \operatorname{SVA}(r=0.88), \operatorname{cSVA}(\mathrm{r}=$ $0.5)$, and $\mathrm{T} 1$ slope $(\mathrm{r}=0.77)$ all increase with age. T1/CL remains stable over all decades $(\mathrm{r}=0.01)$. The ratio between TK and LL also remains stable until the 7 th decade of life $(r=0.8)$. LL is greater than PI but slowly declines until the 5th decade, where it becomes less than PI $(r=0.95)$. SVA remains below $5 \mathrm{~cm}$ until the 7 th decade of life. Before and after age 50, the average LL -55.89, and -48.53 respectively. Similarly, before and after age 50 , the average TK was 27.16 , and 31.73 respectively. Finally, before and after age 50 , the average CL was -10.99 , and -15.53 respectively.

Conclusion: T1 slope/CL is stable over time and important to maintain/target when performing cervical spine surgery. The relationship between TK and LL should be considered when correcting sagittal imbalance. Proposed ideal spinal balance of LL $=\mathrm{PI}+10$ and SVA $<5 \mathrm{~cm}$ are appropriate targets, especially for patients aged 50 and younger.

106 Electrical neuromodulation of the cervical spinal cord facilitates forelimb skilled function recovery in spinal cord injured rats

Tianyi Niu, MD; Nicholas Au Yong, MD, PhD; Yevgeniy Freyvert, MD; Daniel C. Lu, MD, PhD; Monzurul Alam, PhD; Guillermo Garcia-Alias, PhD; Benita Jin, PhD; Jonathan Keyes, PhD; Hui Zhong; Roland R Roy; Yury Gerasimenko; Reggie Edgerton, $\mathrm{PhD}$

Introduction: Enabling motor control by epidural electrical stimulation of the spinal cord is a promising therapeutic technique for the recovery of motor function after a spinal cord injury (SCI). Although epidural electrical stimulation has resulted in improvement in hindlimb motor function, it is unknown whether it has any therapeutic benefit for improving forelimb fine motor function after a cervical SCI.

Methods: We tested whether trains of pulses delivered at spinal cord segments C6 and C8 would facilitate the recovery of forelimb fine motor control after a cervical SCI in rats. Rats were trained to reach and grasp sugar pellets. Immediately after a dorsal funiculus crush at $\mathrm{C} 4$, the rats showed significant deficits in forelimb fine motor control. The rats were tested to reach and grasp with and without cervical epidural stimulation for 10 weeks post-injury. To determine the best stimulation parameters to activate the cervical spinal networks involved in forelimb motor function, monopolar and bipolar currents were delivered at varying frequencies $(20,40$, and $60 \mathrm{~Hz})$ concomitant with the reaching and grasping task.

Results: We found that cervical epidural stimulation increased reaching and grasping success rates compared to the no stimulation condition. Bipolar stimulation $(\mathrm{C} 6 \mathrm{C} 8+$ and $\mathrm{C} 6+\mathrm{C} 8$ ) produced the largest spinal motor-evoked potentials (sMEPs) and resulted in higher reaching and grasping success rates compared with monopolar stimulation (C6 Ref + and C8 Ref +). Forelimb performance was similar when tested at stimulation frequencies of 20, 40, and $60 \mathrm{~Hz}$. We also found that the EMG activity in most forelimb muscles as well as the co-activation between flexor and extensor muscles increased post-injury. With epidural stimulation, however, this trend was reversed indicating that cervical epidural spinal cord stimulation has therapeutic potential for rehabilitation after a cervical SCI.

Conclusion: Epidural spinal cord stimulation can effectively neuromodulate the cervical spinal cord in a way that improves reaching and grasping in rats after an incomplete cervical injury, both in the acute and chronic stages post-injury.
107 Misdiagnosis is a Prevalent Cause of Inappropriate Lumbar Spine Surgery

Anna Wright; Vijay Yanamadala, MD, MBA; Jean-Christophe A. Leveque, MD; Rajiv Sethi, MD

Introduction: The utilization of lumbar spine surgery has increased 7 fold over the past three decades with great heterogeneity in outcomes. We hypothesized that misdiagnosis may be a contributing factor to this heterogeneity and ultimately to the stagnant outcomes.

Methods: We retrospectively reviewed the clinic visits of vascular surgeons, orthopaedic joint surgeons, and neurologists at Virginia Mason Medical Center between January 1, 2010 and July 1, 2016. We identified patients who presented to vascular surgeons, orthopaedic joint surgeons or neurologists with a chief complaint of lower extremity pain. We then identified patients who had previously undergone lumbar spine surgery without improvement of their symptoms as documented in medical records.

Results: 3,114 consecutive patients meeting inclusion criteria were reviewed, 464 presenting to vascular surgery, 1089 to orthopaedic surgery, and 1,561 to neurology. Of 464 patients presenting to vascular surgeons, 111 (19.2\%) had undergone prior spine surgery without improvement. 62 patients $(13.3 \%)$ underwent lumbar decompressive procedures and 27 patients (5.8\%) underwent lumbar fusions. 16 patients underwent multiple lumbar spine surgeries. All of these patients met criteria for diagnosis of vascular claudication by ankle brachial index. Of 1,089 patients who presented to an orthopaedic joint surgeon, 82 patients $(7.6 \%)$ underwent prior spine surgery for their presenting symptom without improvement, with 64 (5.9\%) undergoing lumbar decompressive procedures and $18(1.7 \%)$ undergoing lumbar fusions. All of these patients had radiographic evidence of hip and/or knee osteoarthritis. Of the 1,561 patients presenting to a neurologist, 13 patients $(0.8 \%)$ underwent prior spine surgery without improvement.

Conclusion: A large proportion of patients presenting to other specialists had lumbar spine surgery for presumed spondylosis without improvement in their initial symptoms. They were ultimately diagnosed with the true cause of their symptoms only after undergoing unnecessary surgery. While multiple diagnoses are common, particularly among older patients with multiple co-morbidities, better decision support tools may be necessary to help surgeons manage complex cases. Ultimately, it is imperative to develop ways to prevent unnecessary surgeries which should ultimately be never-events in health care.

108 Patient Satisfaction and Press Ganey Scores for Spine versus Non-Spine Neurosurgery Clinics

Yi-Ren Chen, MD, MPH; Eli Johnson, BS; Carlos Montalvo; Shelley Stratford; Anand Veeravagu, MD; Suzanne Audrey Tharin, MD, PhD; Atman Desai, MD, MA; John K. Ratliff, MD, FACS; Lawrence M. Shuer, MD; Jon Park

Introduction: The Press Ganey survey is a well-established metric for measuring hospital performance and patient satisfaction. We seek to evaluate satisfaction scores in patients seen in spine versus non-spine neurosurgical clinics.

Methods: Retrospective Press Ganey survey review was performed to identify patient demographics and patient visit characteristics from January 1st, 2012 to October 10th, 2017 at Stanford Medical Center. A total of 45 questions from the Press Ganey survey were investigated and grouped in categories addressing physician and nursing care, personal concerns, admission, room, meal, treatment and discharge conditions, visitor accommodations 
and overall clinic assessment. Raw ordinal scores were converted to continuous scores out of 100 for unpaired student t-test analysis. We identified 581 spine clinic patients and 1048 non-spine clinic patients.

Results: Spine clinic patients reported lower satisfaction scores in aggregate (88.2 vs. $90.1 ; \mathrm{p}=0.0014)$, physician $(89.5$ vs. 92.6; $\mathrm{p}=0.0002)$ and nurse care (91.3 vs. 93.4; $\mathrm{p}=0.0038)$, personal concerns (88.2 vs. $90.9 ; \mathrm{p}=0.0009)$, room ( 81.0 vs 83.1 ; $\mathrm{p}=0.0164)$, admission ( 90.8 vs $92.6 ; \mathrm{p}=0.0154)$ and visitor conditions ( 87.0 vs. $89.2 ; \mathrm{p}=0.0148)$, and overall clinic assessment $(92.9$ vs. $95.5 ; \mathrm{p}=0.005)$

Conclusion: This study is the first to evaluate the relationship between neurosurgical spine versus non-spine clinic with regards to patient satisfaction. The spine clinic cohort reported less satisfaction than the non-spine cohort in all significant questions on the Press Ganey survey. We hypothesize that spine clinic patients may have a higher incidence of chronic pain and depression, though our data did not have the granularity to address these issues. Our findings suggest that efforts should be made to further study and improve patient satisfaction in spine clinics.

109 Mesenchymal Stem Cell-Seeded High-Density Collagen Gel for Annular Repair: 6 Week Results from in Vivo Sheep Models

Ibrahim Hussain; Christoph Wipplinger; Stephen Sloan; Rodrigo Navarro-Ramirez, MD, MS; Eliana Kim; Micaella BS Zubkov Weill, Cornell, Medicine; Gernot Lang, MD; Lawrence J Bonassar, PhD; Roger Hartl, MD

Introduction: Our group has previously shown successful in vivo annulus fibrosus (AF) repair in rodents and sheep models using an acellular, riboflavin crosslinked high-density collagen (HDC) gel (1-3). We now report an in vivo study performed in sheep analyzing the effects of seeding allogenic mesenchymal stem cells (MSCs) into this HDC gel for annular repair.

Methods: 15 lumbar intervertebral discs (IVDs) from three sheep were exposed via a lateral pre-psoas approach and randomized into 4 groups: 1) intact $(\mathrm{N}=3) ; 2)$ injury $(3 \mathrm{~mm} \times 1 \mathrm{~cm}$ annulotomy+100mg nucleotomy) $(\mathrm{N}=4) ; 3)$ injury+acellular gel treatment $(\mathrm{N}=4)$; and 4) injury+MSC-seeded gel treatment (106 $\mathrm{MSCs} / \mathrm{mL})(\mathrm{N}=4)$. Sheep were sacrificed at 6 weeks. Disc height index (DHI) and Pfirrmann grading were performed using lateral X-ray and 3T MR images, respectively. Quantitative MRI analyses for nucleus pulposus (NP) area and T2 relaxation time (T2-RT), a surrogate for disc hydration, was also performed. Values were calculated as ratios standardized to healthy controls from the same sheep. Quantitative histologic assessment was also performed using the validated Han scoring system based on cellularity and morphology of the AF and NP (4).

Results: All treated IVDs retained gel plugs on gross assessment and there were no adverse immunologic reactions. For groups 2-4, the following values were obtained: DHI were 0.846 , 0.874 , and 0.96 , respectively $(\mathrm{P}=0.00274)$. Average Pfirrmann grades were $2.5,2.5$, and 1.75 , respectively $(\mathrm{P}=0.00035)$. Average $\mathrm{NP}$ area ratios were $0.868,0.926$, and $0.894(\mathrm{P}=0.636)$. Average T2-RT ratios were $0.784,0.835$, and $0.909(\mathrm{P}=0.0154)$. Average Han scores were $11.5,10$, and $9.5(\mathrm{P}=0.246)$.

Conclusion: Conclusion: MSC-seeded HDC gel treated IVDs demonstrated a statistically significant improvement in DHI, Pfirrmann grade, and T2-RT over other experimental groups. There was a trend for more histologic AF and NP organization with MSC-seeded HDC gel than acellular HDC gel and negative controls. Similar analyses to 16 weeks post-injury are ongoing.
110 Genome-Wide Transposon Mutagenesis Screening Identifies Novel Genetic Drivers and Oncogenic Networks in Spinal Gliomas

\section{Imran Noorani, MD, MRCS}

Introduction: Spinal astrocytomas are the commonest spinal tumor in children, carrying significant morbidity and mortality. Targeted treatments based on more thorough understanding of the biology of this disease are urgently needed. Human tumors harbor many epigenetic and genetic changes, creating difficulties in discerning true genetic drivers. We employ a novel forward genetic screening approach to identify spinal glioma genetic drivers.

Methods: We generated conditional transgenic mice that carry multiple copies of the piggyBac transposon and an EGFRvIII mutation. Targeted PCR demonstrated mobilization of the transposon within the genome of the spinal cord; the transposon has a CAGS promoter to drive gene expression and splice acceptors and polyA repeats to disrupt gene expression. 52 such mice were produced and their spinal cord tumors were collected for genomewide sequencing of their transposon insertion sites and for RNAsequencing.

Results: All 52 mice developed neurological signs, with mean time of death of 8 months. Histology revealed spinal cord astrocytomas between grade II and IV, with immunohistochemical positivity for GFAP, OLIG2 and SOX2. Genome-wide sequencing for common insertion sites (CIS) of the transposon revealed 302 significantly targeted CIS in multiple tumors $(\mathrm{p}<0.0001)$. Known human glioma genetic drivers CDKN2A, NF1 and PTEN were amongst the most frequent CIS. Network analysis revealed significant interactions between the 302 CIS genes $(\mathrm{p}<0.001)$, and novel genes cooperating with EGFR in spinal gliomagenesis included the Wnt signaling genes Csnk1g3, APC2 and SMARCA2. Global transcriptomic profiling of these tumors showed significant differential expression of neural development genes, yielding tumorigenic mechanistic insights.

Conclusion: We show EGFRvIII is sufficient to initiate spinal astrocytoma formation, but requires additional genetic drivers for tumor evolution. We have comprehensively identified known and novel genes important in this process, highlighting differences in genetics between brain and spinal astrocytomas, and suggest new therapeutic strategies based on these unique oncogenic networks.

111 Decreasing Radiation Exposure in Minimally Invasive Spinal Surgery with Ultra-Low Radiation Imaging and Image Enhancement: A Prospective Cohort Study of Consecutive Patients

Jakub Godzik, MD, MSc; Gautam Nayar; William D. Hunter, MD; Luis Manuel Tumialan, MD

Introduction: Visualization of the anatomy in minimal access spinal surgery is limited and dependent on radiographic imaging. Conventional fluoroscopy in minimally invasive surgery has led to substantial increases in radiation exposure. Ultra-low radiation imaging (ULRI) with image enhancement is another alternative. Our objective was to compare radiation exposure between standard-dose (SDF) and ULRI fluoroscopy with image enhancement in patients undergoing lateral transpsoas lumbar interbody fusion (XLIF), percutaneous screws placed as part of XLIF procedure (XLIF-PS), or minimally invasive transforaminal lumbar interbody fusion (MIS-TLIF).

Methods: Sixty-one consecutive patients under a single surgeon were prospectively enrolled. SDF was used in 30 cases and ULRI with image enhancement was used in 29 cases. All radiation exposure ( $\mathrm{mGy}$ ) was recorded from the $\mathrm{C}$-arm fluoroscope; radia- 
tion exposure was compared between imaging modalities both per case and per surgical level.

Results: Twenty-one patients underwent XLIF using SDF, and 11 patients using ULRI; 10 patients underwent MIS-TLIF using SDF, and 18 patients using ULRI. There were no significant differences in age, BMI, or number of levels $(p>0.65)$ between SDF and ULRI. There were significant decreases in radiation exposure per level for XLIF (119.8 vs. $36.4 \mathrm{mGy}, \mathrm{p}<0.0001)$, XLIF-PS (47.1 vs. $15.4 \mathrm{mGy}, \mathrm{p}<0.0001)$, and MIS-TLIF (121.6 vs. $24.4 \mathrm{mGy}, \mathrm{p}=0.003$ ) when utilizing ULRI compared to SDF, corresponding to $69.6 \%, 67.3 \%$, and $79.9 \%$ reduction in radiation exposure respectively. Total radiation dosages per case were also decreased in XLIF (148.8 vs. $46.5 \mathrm{mGy}, \mathrm{p}<0.0001)$, XLIFPS (220.3 vs. $75.4 \mathrm{mGy}, \mathrm{p}<0.0001)$, and MIS-TLIF (141.8 vs. $27.0 \mathrm{mGy}, \mathrm{p}=0.004)$. No instances of postoperative neurological decline or revision surgery were observed.

Conclusion: ULRI with image enhancement has the capacity to significantly decrease radiation exposures in minimally invasive procedures without compromising visualization of the anatomy or the safety of the procedure.

112 The Applicability of Cervical Sagittal Vertical Axis, Lordosis, and T1-slope on Pain and Disability Outcomes after Anterior Cervical Discectomy and Fusion

Darryl Lau, MD; Anthony DO Digorgio; Andrew Kai-Hong Chan, MD; Michael S. Virk, MD, PhD; Dean Chou, MD; Erica Fay Bisson, MD, MPH, FAANS; Praveen V. Mummaneni, MD

Introduction: Understanding what influences pain and disability following anterior cervical discectomy and fusion (ACDF) is critical. This study examines the timing of clinical improvement and identifies factors associated with worse outcomes.

Methods: Consecutive adult patients were enrolled in a prospective outcomes database from two academic centers from 2013 to 2016. Demographics, surgical details, x-rays, arm and neck pain (visual analogue scale (VAS)), and disability (neck disability index (NDI) and EQ5D) were reviewed. Multivariate analysis was used.

Results: A total of 186 patients were included. Mean age was 55.4 years and $45.7 \%$ had myelopathy. Preoperative cervical sagittal vertical axis (cSVA), cervical lordosis (CL), and T1-slope was $24.9 \mathrm{~mm}, 10.4$ degrees, and 28.3 degrees. ACDF was performed at 1-, 2-, and 3-levels in $47.8 \%, 41.9 \%$, and $10.2 \%$ of patients. Preoperative neck and arm VAS were 5.7 and 5.4. NDI and EQ5D scores were 22.1 and 0.5 . There was significant improvement in all outcomes at 3 -months $(p<0.001)$ and 12 -months $(p<0.001)$. At 3 months: neck VAS (3.0), arm VAS (2.2), NDI (12.7), EQ5D (0.7) and at 12 months: neck VAS (2.8), arm VAS (2.3), NDI (11.7), EQ5D (0.8). Improvements occurred within the first 3-months; there was no significant difference in outcomes between the 3-month and 12-month mark. There was no correlation between cSVA, CL, or T1-slope to all outcome endpoints. The most consistent independent preoperative factor associated with worse outcomes was high VAS and severe NDI $(\mathrm{P}<0.001)$. Similar findings were seen with worse NDI and EQ5D scores $(p<0.001)$. A significant linear trend of worse NDI and EQ5D at 3 and 12 months were associated with worse baseline scores.

Conclusion: ACDF is effective in improving pain and disability, and improvement occurs within 3-months. cSVA, CL, and T1-slope do not influence outcomes following ACDF surgery. Worse preoperative pain and disability was an independently associated with worse outcomes.
113 Development of Proximal Junctional Kyphosis After Minimally Invasive Lateral Anterior Column Realignment for Adult Spinal Deformity

Shashank V Gandhi, MD; Jacob Januszewski, DO; Konrad Bach, MD; Andrew C. Vivas, MD; Jason Michael Paluzzi; Adam S. Kanter, MD; David O. Okonkwo, MD, PhD; Zachary J. Tempel, MD; Nitin Agarwal, MD; Juan S. Uribe, MD, FAANS

Introduction: Development of proximal junctional kyphosis (PJK) after correction of adult spinal deformity (ASD) undermines sagittal alignment. Minimally invasive anterior column realignment (ACR) is a powerful tool to obtain robust correction of ASD; however, long-term PJK rates are still unknown. This study aims to characterize PJK after utilization of ACR in ASD correction.

Methods: A retrospective multi-institution cohort analysis per STROBE criteria was conducted of all patients who underwent ACR for ASD from 2010-2015. All patients obtained preoperative and follow-up upright full radiographs, assessing for spinal alignment and development of PJK. Patients without proper imaging or at least 1-year follow up were excluded.

Results: Total of 41 of 73 patients who underwent ACR met inclusion criteria, with a mean follow-up of 20.4 months. There was a significant improvement of all spinopelvic parameters. PJK developed in $36.6 \%$ patients, of which $19.5 \%$ progressed to PJF. The incidence of PJK increased with the addition of posterior column osteotomy (PCO): $42.9 \%$ versus $30 \%$. There was a higher PJK rate when the upper-instrumented vertebra was located at T10-L1 versus L2-L4 (52.4\% versus $20 \%, \mathrm{p}=0.031)$. No patients with only lateral transpsoas lumber interbody fusions (LLIF) developed PJK.

Conclusion: The incidence of PJK after minimally invasive ACR is slightly lower than reported after open surgery but greater than in LLIF only. The rate increases with concurrent PCO utilization. The PJK rate is higher in constructs that cross the TL junction. Elderly patients are at an increased risk of PJK, suggesting a need for age-appropriate correction goals.

114 Factors Associated with Venous Thromboembolic Events in Spine Surgery Patients in the Intensive Care Setting: A Single-Institution Experience with 1269 Consecutive Patients

Michael Cloney, MD; Jack Goergen, BS; Benjamin Hopkins, BS; Jonathan Tad Yamaguchi, BS; Nader S. Dahdaleh, MD

Introduction: Venous thromboembolic events (VTE) are a common cause of morbidity and mortality after spine surgery. Patients admitted to the ICU following spine surgery are a subgroup of patients who are at higher risk of complications, including VTE. We identified factors independently associated with VTE in this unique patient population.

Methods: We retrospectively analyzed 6869 patients who underwent spine surgery at our institution, of whom 1269 were admitted to the ICU. For ICU patients, we identified demographic, clinical, and procedural factors independently associated with VTE during three time periods: during the ICU admission, after leaving the ICU, at any point during the first 30 postoperative days.

Results: There was a difference in time-to-VTE based on the type of surgery being performed, with osteotomy patients having a prolonged median time-to-VTE. A history of DVT, PE, and prior IVC filter placement were associated with having a DVT or PE during more than one of the three time periods analyzed. DVT in the ICU was associated with undergoing longer surgeries (OR $1.05, \mathrm{p}=0.002$ ), and there was a trend toward significance for fractures (OR 3.09, $\mathrm{p}=0.051)$. DVT after leaving the ICU was associ- 
ated with fusion (OR 0.36, $\mathrm{p}=0.045$ ) and osteotomy (OR 3.14, $\mathrm{p}=0.045)$. PE during the ICU stay was associated with fractures (OR 7.02, $\mathrm{p}=0.040$ ) and scoliosis correction (OR 7.78, $\mathrm{p}=0.024)$. Prophylactic anticoagulation was negatively associated with $\mathrm{PE}$ during the ICU stay (OR $0.16, \mathrm{p}=0.031$ ). Men were less likely to develop a PE after leaving the ICU (OR 0.12, p=0.006).

Conclusion: Patients admitted to the ICU following spine surgery are typically in poorer health, and are undergoing high-risk surgeries. Time-to-VTE varies between types of surgeries. Some factors are independently associated with VTE events throughout the 30-day postoperative period, while others are associated with VTE specifically during the ICU stay or after leaving the ICU.

115 Patient Demographic Factors Which Affect Patient Reported Outcomes (PRO) Completion 1 Year After Spine Surgery: Analysis from the Michigan Spine Surgery Improvement Collaborative (MSSIC)

Hesham Mostafa Zakaria, MD; Michael Bazydlo, MS; Shujie Xiao, MS; Lonni Schultz, PhD; David Nerenz, PhD; Muwaffak Abdulhak, MD, FRCS; Jason M. Schwalb, MD, FAANS, FACS; Victor W. Chang, MD; For the MSSIC Investigators

Introduction: The Michigan Spine Surgery Improvement Collaborative (MSSIC) is a statewide multicenter quality improvement collaborative.1 Since missing data from large registries may adversely affect results, 2,3 we sought to identify patient demographic features that affect the rate of complete follow-up of patient-reported outcomes (PROs) at 90 days and 1 year.

Methods: 24404 patients from the 26 MSSIC hospitals who had lumbar (17813 patients) or cervical (6591 patients) spine surgery were studied. Multivariable logistic regression models of patient demographics were constructed to identify risk factors for incomplete PRO follow-up.

Results: Patients $=65$ yo were more likely than $<65$ yo to provide PRO information at $90 \mathrm{~d}$ and $1 \mathrm{yr}(\mathrm{p}<0.001)$. Female patients were more likely to respond at $90 \mathrm{~d}(\mathrm{p}<0.001)$ and $1 \mathrm{yr}(\mathrm{p}=0.040)$. Compared to those with a high school education, patients with less than high school education were less likely to respond at $90 \mathrm{~d}$ $(\mathrm{p}=0.003)$ and $1 \mathrm{yr}(\mathrm{p}<0.001)$, and patients with some college education or more were more likely to respond at the same time points $(\mathrm{p}<0.001)$. Caucasians and African Americans had no difference in response rate at $90 \mathrm{~d}$ and $1 \mathrm{yr}$, but both had increased response as compared to other ethnicities at $90 \mathrm{~d}$ and $1 \mathrm{yr}(\mathrm{p}=0.013, \mathrm{p}=0.043$, respectively). Contacting the patient via web provided a decrease response at $90 \mathrm{~d}$ and $1 \mathrm{yr}$ as compared to mail $(\mathrm{p}=0.003, \mathrm{p}<0.001)$ and calling $(\mathrm{p}<0.001, \mathrm{p}=0.03)$. Employment status, private vs public insurance, household income, and distance from surgical site did not affect response rate.

Conclusion: A multivariate analysis from a large, multicenter, prospective database identified modifiable and unmodifiable patient demographic factors that can affect the rate of PRO follow-up. Older, female, and more educated patients were more likely to have increased PRO completion rates at 90d and 1yr. This data can be helpful for imputing missing PRO data, and help overcome challenges of patient non-compliance in large prospective data sets.

\section{Tumors}

116 Development of a Frailty Index for Primary Spinal

A. Karim Ahmed; C. Rory Goodwin, MD, PhD; Rafael De la Garza-Ramos, BA; Rachel C. Kim, BS; Nancy A Abu-Bonsrah, MD; Camilo A. Molina, MD; Eric W. Sankey, MD; Risheng Xu, AB, AM, MD, PhD; Daniel M. Sciubba, MD

Introduction: The concept of 'Frailty,' i.e. decreased physi- ologic reserve and increased vulnerability to stressors beyond what is expected for normal aging is associated with increased risk of morbidity and mortality. The objective of this study was to develop a preoperative frailty index (STFI) for patients undergoing surgery for primary spinal column tumors that predicts morbidity, mortality, and length of stay.

Methods: The Nationwide Inpatient Sample database from 2002 to 2011 was used to identify patients who underwent surgery for a primary spinal tumor. The STFI, consisting of nine items, was applied to each patient. Patients were characterized as not frail (0), mildly frail, moderately frail (2), and severely frail (=3).

Results: A total of 1,589 patients met inclusion criteria. The overall major complication rate was $10.6 \%$. Compared to patients without frailty, patients with mild (OR 3.83; 95\% CI, 2.63- 5.58), moderate (OR 6.80; 95\% CI, 4.10- 11.3), and severe frailty (OR 13.05; 95\% CI, 6.34 - 26.87) had significantly increased odds of complication development (all $\mathrm{p}<0.001$ ). The mean length of stay was $6.4 \pm 0.2$ days, 9.8 \pm 0.6 days, $14.4 \pm 1.7$ days, and $18.3 \pm 2.6$ days for patients without frailty, with mild frailty, with moderate frailty, and with severe frailty, respectively ( $\mathrm{p}<0.05$ between all groups).

Conclusion: Compared to patients without frailty, patients with mild, moderate, and severe frailty had significantly increased odds of developing post-operative complications. A systematic evaluation of pre-operative frailty should play a key role in decisionmaking for patients undergoing surgery for primary spinal tumors.

117 Preliminary Return to Work Data from the MultiCenter Prospective, Randomized CSM-S Study: Approach Matters

Jian Guan, MD; Nisreen Ghogawala; Melissa Dunbar, BS; Praveen V. Mummaneni, MD; Adam S. Kanter, MD; Erica Fay Bisson, MD, MPH, FAANS; K Daniel Riew, MD; Robert F. Heary, MD; Edward C. Benzel, MD; Michael P. Steinmetz, MD; James S. Harrop, MD, FACS; Marjorie C. Wang

Introduction: Lost productivity related to cervical spondylotic myelopathy (CSM) has significant potential economic impact. Little information exists on return to work rates (RTW) following CSM surgery.

Methods: Using data from the multi-center prospective, randomized CSM-S study, we performed a three-way cohort RTW analysis of patients who were working preoperatively. Patients were randomized to ventral surgery (anterior cervical decompression and fusion $[\mathrm{VF}]$ ) or dorsal surgery (posterior fusion $[\mathrm{PF}]$ or laminoplasty [LP]). Age, gender, number of stenotic levels, preoperative SF-36 PCS/MCS, NDI, mJOA, and EQ5D scores, and postoperative hard-collar use were examined. RTW was evaluated at one, six, and twelve months.

Results: Seventy-six patients were analyzed, with 38 undergoing VF, $26 \mathrm{PF}$, and $12 \mathrm{LP}$. Mean age was 59.2 \pm 7.4 years and was similar among groups $(\mathrm{p}=0.16)$. Baseline $\mathrm{mJOA}$ and other patientreported outcome scores were also similar. Stenotic levels differed significantly between groups, with VF patients having $2.5 \pm 0.5$ levels, compared to $3.1 \pm 0.7$ for $P F$ and $3.1 \pm 0.9$ for $L P(p<0.001)$. Hard-collar utilization was not significantly different between VF $(36.8 \%)$ and PF $(50 \%, p=0.30)$, although no LP patients utilized a hard-collar. RTW at one year was significantly higher for LP $(91.7 \%)$ and VF $(89.5 \%)$ compared to PF $(50 \%, \mathrm{p}<0.001)$. LP had significantly faster RTW than VF $(2.1 \pm 1.0$ vs. $4.3 \pm 3.4$ months, $\mathrm{p}=0.04)$ and $\mathrm{PF}(2.1 \pm 1.0$ vs. $4.5 \pm 3.3$ months, $\mathrm{p}=0.03)$. Hard-collar use was similar in patients unable to RTW (44.4\%) and those who were $(32.8 \%, \mathrm{p}=0.37)$, though time to RTW was longer in hardcollar patients $(5.3 \pm 3.7$ vs. $3.3 \pm 2.7$ months, $\mathrm{p}=0.02)$. Multipleregression analysis identified surgical approach as an independent predictor of RTW. 
Conclusion: Most patients undergoing CSM surgery achieve RTW status. Among the CSM-S study cohort, PF patients were $40 \%$ less likely to RTW within one year than VF or LP patients. Surgical approach was an independent predictor for RTW status although a major factor was the utilization of a hard-collar.

118 Effect of Body Mass Index as a Continuous Variable on 30-Day Postoperative Complications in Posterior Lumbar Fusion: An Analysis of the American College of Surgeons National Surgical Quality Improvement Program MD

Juneyoung L Yi, MD, MSPH; Ali Jalali, MA; Bornali Kundu,

Introduction: Studies have shown a negative association between high BMI and postoperative outcomes for posterior lumbar fusion. However, BMI is typically analyzed as a categorical variable. Recent studies analyzing BMI as a continuous variable for hip and knee arthroplasties demonstrated non-linear effects. This study uses ACS NSQIP datasets to evaluate the effect of BMI as a continuous variable on 30-day postoperative complications for posterior lumbar fusions.

Methods: Spline analyses utilizing logistic regression models were performed to assess the validity of a BMI threshold of 40 $\mathrm{kg} / \mathrm{m} 2$. BMI was included as two exposures to evaluate for its overall effect as well as the marginal effect of BMI $>40 \mathrm{~kg} / \mathrm{m} 2$. In a second analysis, univariate restricted cubic splines were used to determine the presence of non-linear effects of BMI associated OR's and probabilities of postoperative complications.

Results: BMI was a significant predictor of DVT/thrombophlebitis $(\mathrm{OR}=1.052 ; 95 \%$ CI $1.002,1.105)$ and superficial SSI $(\mathrm{OR}=1.048 ; 95 \%$ CI 1.003, 1.096). The marginal effect of BMI $>40 \mathrm{~kg} / \mathrm{m} 2$ was a significant predictor of cardiac arrest $(\mathrm{OR}=1.288 ; 95 \%$ CI $1.018,1.629)$, MI $(\mathrm{OR}=1.224 ; 95 \%$ CI 1.037 , 1.446), pneumonia (OR=1.146; $95 \%$ CI $1.013,1.295)$, and wound dehiscence $(\mathrm{OR}=1.280 ; 95 \%$ CI $1.102,1.487)$. Probabilities of cardiac arrest, MI, ventilator use, unplanned intubation, superficial SSI, deep SSI, and wound dehiscence demonstrated positive linear trends after BMI $>30 \mathrm{~kg} / \mathrm{m} 2$. OR's for cardiac arrest, ventilator use, unplanned intubation, superficial SSI, and deep SSI also demonstrated positive linear trends after BMI $>30 \mathrm{~kg} / \mathrm{m} 2$. Probabilities and OR's for pneumonia and RBC transfusion demonstrated negative linear trends for $\mathrm{BMI}<30 \mathrm{~kg} / \mathrm{m} 2$ and positive linear trends for BMI $>30 \mathrm{~kg} / \mathrm{m} 2$.

Conclusion: When analyzed as a continuous variable, BMI demonstrates a non-linear effect and is a significant predictor of 30-day postoperative complications for posterior lumbar fusions.

119 Analysis of Outcomes and Cost of Inpatient and Ambulatory Anterior Cervical Disk Replacement Using a State-level Database

Allen L Ho, MD; David Arnold Purger, MD; Arjun Vivek Pendharkar, MD; Eric S Sussman, MD; Anand Veeravagu, MD; John K. Ratliff, MD, FACS; Atman Desai, MD, MA

Introduction: Anterior cervical procedures are a promising candidate for transition to the outpatient setting due to their wellstudied low complication/morbidity profile. Utilizing populationlevel database data, we compare clinical outcomes and cost associated between inpatient and ambulatory setting cervical artificial disk replacement (ADR).

Methods: Demographics, comorbidities, emergency department (ED) visits, readmissions, reoperation rates, and 90-day charges were retrospectively analyzed for patients undergoing elective ADR in CA, FL, and NY from 2009-2011 in State
Inpatient and Ambulatory Databases.

Results: A total of 1,789 index ADR procedures were identified in the inpatient database (SID) compared to 370 procedures in the ambulatory cohort (SASD). The groups were further stratified into groups of Charlson Comorbidity Index of zero or nonzero to adjust for perioperative comorbidities between groups. Ambulatory ADR patients presented to the emergency department 19 times $(5.14 \%)$ within 30 days of the index procedure. Inpatient ADR patients had an ED visit rate of $4.2 \%$. 4 unique patients underwent readmission within 30 days in the ambulatory ADR cohort (1\% total) compared to $2.2 \%$ in the inpatient ADR group. No ambulatory ADR patients underwent a reoperation within 30 days. Of the inpatient ADR group 6 unique patients underwent reoperation within 30 days $(0.34 \%, \mathrm{CCI}=0 \quad 0.28 \%, \mathrm{CCI}>0.0 .6 \%)$. There was no significant difference in ED visit rate, inpatient readmission rate nor reoperation rates within 30 days of the index procedure between outpatient or inpatient ADR. Outpatient ADR is non-inferior to inpatient ADR in all clinical outcomes. Direct cost was significantly lower in the outpatient ADR group $(\$ 11059$ vs $17033, p<0.001)$. 90-day cumulative charges were significantly lower in the outpatient ADR group (mean $\$ 46404.03$ vs $\$ 80055$, $\mathrm{p}<0.0001)$.

Conclusion: ADR can be performed in an ambulatory setting with comparable morbidity, readmission rates, and lower costs, to inpatient ADR.

120 Implementation of a Standardized Multimodal Analgesia Protocol Improves Pain-related Outcomes, Decreases Hospital Stay and Reduces Opioid Consumption After Posterior Lumbar Spinal Fusion Surgery

Corey Tyler Walker, MD; Tyler Scott Cole, MD; David Gullotti; Virginia Prendergast; Jakub Godzik, MD, MSc; Alex Whiting, MD; Jay D. Turner, MD, PhD

Introduction: Optimized postoperative pain control after spinal fusion surgery may lead to an improvement in clinical outcomes. An evidence-based, multi-disciplinary quality improvement initiative was implemented to standardize pain treatment following neurosurgical procedures at our institution with the goal of improving clinical outcomes and patient satisfaction.

Methods: A retrospective chart review was conducted to evaluate pain-related outcomes after posterior lumbar fusion procedures at a single institution. We compared patients treated six months preceding and the six months following the implementation of the standardized pain protocol.

Results: A total of 118 pre- and 128 post- implementation patients were identified. The cohorts were well matched with no differences in sex, age, surgical duration, number of segments fused, pre-operative pain level or baseline physical status (all $\mathrm{p}>0.05$ ). Average patient-reported pain scores significantly improved in the first 24 hours post-operatively (5.8 versus 4.6 , $\mathrm{p}<0.001$ ) and 24 to 72 hours post-operatively (4.9 versus 3.9, $\mathrm{p}<0.001)$ following use of the pain protocols. Likewise, maximum pain scores during these periods, as well as time to achieving appropriate pain control were also significantly improved. Further, a statistically significant decrease in opioid consumption during the first 72 hours was seen (129 versus 87 MME (morphine milligram equivalents), $\mathrm{p}=0.001$ ). Patients in the post-implementation cohort also had a significantly decreased length of hospital stay (4.8 versus 4.1 days, $\mathrm{p}=0.001$ ).

Conclusion: Implementation of an evidence-based, standardized multi-modal pain protocol results in tangible improvements following posterior lumbar spinal fusion, including reduction in pain scores, opioid consumption, and length of hospital stay. 
121 Preoperative Narcotic Consumption - Does It Affect Outcome?

Lee Onn Chieng, MD; Karthik Madhavan, MD; Michael Y. Wang, MD, FACS; Steven Vanni, DO, DC

Introduction: Opioid dependence is relatively common in patient with chronic back pain. In this study, we aim to establish the relationship between preoperative opiod usage and outcome of surgery.

Methods: We retrospectively analysed our prospectively maintained spine database of 575 patients. 265 patients were included after applying strict criteria. Demographics and outcome data were collected. Univariate and multivariate analysis were conducted.

Results: $49 \%$ of our patient population received narcotic prior to spine surgery. They have share similarity in demographics which eliminate confounders in this studies. They typically present with higher preoperative VAS score (mean: 7.8, $\mathrm{p}=0.001$ ) and higher postoperative VAS score ( 4.0 vs $2.9, \mathrm{p}=0.001)$. Those patients who did not consume narcotic has lower ODI (14.8 vs $30.6, \mathrm{p}<0.001)$ and EQ50 (0.7 vs 1.3, p=0.6). Interestingly, there were more patients in non-preop opiod group being discharged to skilled nursing facility than the opiod group ( $80 \%$ vs $20 \%$ ). Nontheless higher 30 -days readmission rate $(5.4 \%$ vs $3.7 \%$, $\mathrm{p}=0.23)$ and 30 -days reoperation rate $(5.4 \%$ vs $1.5 \%, \mathrm{p}=0.14)$ were evidenced in opiod group.

Conclusion: In this study, we demonstrated worse outcome for those patient consume narcotic preoperatively. A strong effort of avoiding prescribing narcotic is desperately needed. In our institution, we have recently introduced Enhanced Recovery After Surgery protocol which has proved to improve outcome and reduce narcotic requirement.

122 Use of Polyetheretherketone Interbody Devices for One-level Anterior Cervical Discectomy and Fusion Results in a Five-fold Higher Rate of Pseudarthrosis Compared to Structural Allograft

Katie L Krause, MD, PhD; James T Obayashi, BS; Kelly Bridges, MD; Ahmed M.T. Raslan, MBBS, MCh; Khoi Duc Than, MD

Introduction: Anterior cervical discectomy and fusion (ACDF) is a common surgical procedure used to treat radiculopathy and myelopathy. Common interbody graft options include: 1) structural allograft and 2) polyetheretherketone (PEEK) devices, which have gained popularity due to their radiolucent properties and elastic modulus similar to bone. The use of PEEK devices results in higher hospital billing than structural allograft, which may drive graft selection. In this study, we examined the occurrence of pseudarthrosis of PEEK devices vs. structural allograft in patients who underwent a one-level ACDF.

Methods: Consecutive patients $(n=127)$ who underwent a one-level ACDF over a period of 5 years, and had at least 1 year of radiographic follow-up at our tertiary care center, were retrospectively reviewed. Data on patient age, sex, body mass index (BMI), tobacco use, pseudarthrosis, and re-operation rate for pseudarthrosis were collected. Data was analyzed with a Pearson's chi square test.

Results: Of the 127 patients, 56 had PEEK implants, and 71 had structural allograft implants. There were no baseline differences between age, sex, or BMI in the two groups. There were 29/56 (51\%) patients with PEEK implants who demonstrated radiographic evidence of pseudarthrosis, compared to 7/71 (10\%) patients with structural allograft $(\mathrm{p}<0.001$, OR 9.82; CI: 3.83625.139). Seven patients with PEEK implants required a revision operation for pseudarthrosis, compared to 1 patient with structural allograft ( $\mathrm{p}=0.01$, OR 10.00; CI: 1.192-83.884). There was no statistical difference between the prevalence of tobacco use in patients with either a PEEK device or structural allograft $(\mathrm{p}=$ $0.586)$.

Conclusion: This study provides strong evidence that the use of PEEK interbody devices in a one-level ACDF results in an alarmingly high rate of radiographic pseudarthrosis and need for revision surgery. Surgeons should be aware of this data when deciding on interbody graft options, and reimbursement policies should take into account these discrepancies in outcomes.

123 Modern Targeted Adjuvant Therapies Improves Postoperative Outcomes in Metastatic Renal Cell Carcinoma to the Spine

Ganesh Shankar, MD, PhD; Laura Van Beaver; Bryan D. Choi, MD; Vijay Yanamadala, MD, MBA; Kevin, MD Oh; John H. Shin, MD

Introduction: Modern medical management of metastatic renal cell carcinoma (RCC) includes therapies targeting growth pathways (PDGFR, mTOR), angiogenesis, and immunotherapy. We hypothesized that patients with spinal metastases would continue to benefit from postoperative initiation of these therapeutics despite presenting with advanced stages of disease.

Methods: The study was approved by the IRB at MGH. Baseline characteristics were recorded for patients, including ASIA and performance scores, Fuhrman grade, adjuvant therapies, progression free (PFS) and overall survival (OS) following the respective procedures. Pre-procedural imaging was reviewed to assign Spinal Instability Neoplastic Score (SINS) and epidural spinal cord compression (ESCC) classification. Kaplan-Meier survival analysis was performed using R statistical software.

Results: We identified 32 patients with metastatic RCC to the spine treated at MGH between 2010-2017 by surgical resection with $72 \%$ also receiving postoperative radiosurgery. Instrumented stabilization was performed in $81 \%$ of patients for ESCC 2-3 and median preoperative SINS 12. Preoperative embolization was performed in $62 \%$ of patients with lower intraoperative blood loss $(500 \pm 310 \mathrm{cc}$ vs $1000 \pm 510 \mathrm{cc})$. Increased progression free (160 \pm 91 days vs $112 \pm 50$ days) and overall survival $(913 \pm 317$ days vs $466 \pm 255$ days) following surgery was noted in patients who received postoperative targeted therapies, including inhibitors of PDGFR (100\%), VEGF (50\%), mTOR (33\%) and PD-L1 $(11 \%)$ initiated at a median of 63 days (28-1254 days) postoperatively. All patients noted postoperative improvement in pain and neurologic function. Postoperative complications included one wound infection and one pulmonary embolus requiring anticoagulation.

Conclusion: Postoperative outcomes for metastatic RCC without targeted therapies in this cohort are similar to those reported in earlier series prior to the adoption of these adjuvants. However, we observe a significant increase in survival when modern targeted therapies are administered postoperatively. This has implications on optimizing postoperative multimodal management and also the preoperative evaluation of patients with systemic disease who may have previously been deemed poor surgical candidates. 
200 The Impact of Adding Posterior Instrumentation to Trans-psoas Lateral Fusion: A Systematic Review and Metaanalysis

Mohammed Ali Alvi, MD; Redab Alkhataybeh; Waseem Wahood, MS; Panagiotis Kerezoudis; Sandy Goncalves; M. Hassan Murad, MD, MPH; Mohamad Bydon, MD

Introduction: Trans-psoas Lateral interbody fusion, commonly referred to by one of its trade-names, "XLIF" (Extreme Lateral Interbody Fusion) is one of the lateral minimally invasive approaches for lumbar spine surgery. Most surgeons insert the interbody cage laterally and then insert pedicle or cortical screw and rod instrumentation posteriorly. However, stand-alone cages have also been employed to avoid posterior instrumentation. To the best of our knowledge, the literature on comparison of the two approaches is sparse.

Methods: We performed a systematic review and meta-analysis of available literature on trans-psoas lateral interbody fusion. We compared patients undergoing trans-psoas stand-alone fusion (TP) with those undergoing trans-psoas fusion with posterior instrumentation (TPP).

Results: A total of 29 studies with 1480 patients were included. Four-hundred and five patients underwent TPP, and 956 patients underwent TP. Mean age ranged from 59 to 68 years in the TP group, and 50 to 68 years in the TPP group. The incidence of reoperation was found to be higher for TP $(0.08,95 \%$ CI 0.04 to 0.11$)$ compared to TPP $(0.03,95 \%$ CI 0.01 to 0.06$)$ $(\mathrm{p}=0.057)$. Similarly, mean incidence of reoperation was found to be greater in the TP. The incidence of cage movement was found to be greater in TP $(0.18,95 \%$ CI 0.10 to 0.26$)$ compared to TPP $(0.03,95 \%$ CI 0.01 to 0.05$)(\mathrm{p}<0.001)$. ODI, VAS scores and postoperative transient deficits were found to be comparable between the 2 groups.

Conclusion: Our results seem to suggest that addition of posterior instrumentation to transpsoas fusion is associated with decreased re-operations and cage movements. The results of previous systematic reviews and meta-analyses should be re-evaluated in the light of these results which seem to suggest that higher reoperation and subsidence rates may be due to the employment of stand-alone technique

201 Predictive Factors in Achieving Indirect Decompression in Minimally-invasive Lateral Lumbar Interbody Fusion

Noojan Kazemi, MD; Arunprasad Gunasekaran; Reem Elwy, MBBCh; Manoj Kumar, MD

Introduction: It is unknown whether the degree of indirect decompression achieved is affected by lumbar level and/or location of the cage in the disc space in lateral lumbar interbody fusion (LLIF). The purpose of this study is to identify any association between interbody cage location in the disc space or lumbar level and the degree of indirect decompression achieved.

Methods: A review of 54 disc levels in consecutive patients who underwent LLIF with pre and immediate post-op MRI was performed. Primary outcome variables included: 1) Change in intervertebral disc height at the level of the posterior longitudinal ligament (PLL) 2)Change in mid-sagittal antero-posterior (AP) canal diameter and axial thecal sac area 3 ) the ratio of mean change in canal diameter relative to increase in intervertebral disc height, termed the indirect decompression ratio; and 4) the location of cage placement as a ratio of the posterior aspect of the cage relative to the total AP length of the disc space

Results: 54 disc levels (L1/2 -2; L2/3 - 11; L3/4 - 19; L4/5 22) were assessed from consecutive patients from October 2015 to October 2017. Cage location varied from 0.09 to 0.49 along the width of the disc space as a ratio. There was a statistically significant relationship between the cage location and the indirect decompression ratio $(\mathrm{p}<0.001$; ci $0.58-1.27)$. The indirect decompression ratio (range 0.04-3.4) was calculated for each level (L1/21.29; L2/3 1.14; L3/4 1.10; L4/5 1.01).

Conclusion: There appears to be a significant relationship between the location of the interbody cage and the degree of indirect decompression achieved. For each descending level both the indirect decompression ratio as well as the relative improvement of thecal sac area decreased implying greater disc height change needed to achieve the same improvement in indirect decompression. The indirect decompression ratio is proposed to quantify the improvement in canal diameter adjusted for the change in disc height achieved during LLIF.

202 Surgical Feasibility and Results of Oblique Lateral Interbody Fusion at L5-S1 Level

Alisson R Teles, MD; W. Bradley Jacobs, MD; Fred Nicholls; Peter Lewkonia; Roger Cho; Kenneth Thomas, MD, MHSc; Ganesh Swamy

Introduction: Oblique lateral interbody fusion at L5-S1 level (OLIF51) is a recently introduced less-invasive technique that presents several clear advantages over open surgical approaches for this level. The literature lacks information on appropriate patient selection criteria for OLIF51 and predictors of successful execution. Our objective was to identify predictors for feasibility of OLIF51.

Methods: Retrospective review of consecutive cases of OLIF51 performed between 2014 and 2017. Failure was defined as a major vascular injury or abortion of the procedure due to difficulty of vessel mobilization/exposure of the disc space. Potential predictors for failure were age, gender, surgical indication, previous abdominal surgery, position of the iliocaval junction (ICJ), position of the left common iliac vein (LCIV), absence of fat behind the LCIV, flattening of the LCIV, presence of a large osteophyte at L5-S1. Anatomical features were assessed in the preoperative MRI of the lumbar spine. Bivariate and multiple regression analyses were performed to identify predictors for unfeasibility of OLIF51 using SPSS.

Results: A total of 62 OLIF51 were attempted during the study period, $53(87.1 \%)$ were completed and $8(12.9 \%)$ were aborted intraoperatively. The rate of vascular injury was $6.5 \%(\mathrm{~N}$ $=4)$. In the bivariate analyses, the risk factors for unfeasibility of OLIF51 were position of the LCIV $(\mathrm{P}<0.0001)$, low ICJ (P $=0.001)$, absence of fat behind the LCIV $(\mathrm{P}=0.018)$, and male gender $(\mathrm{P}=0.024)$. Logistic regression demonstrated that medial position of the LCIV (A-OR $=26.29, \mathrm{P}=0.001)$ and male gen$\operatorname{der}(\mathrm{A}-\mathrm{OR}=9.68, \mathrm{P}=0.019)$ were independent predictors for unfeasibility of OLIF51. Median distance of LCIV from midline was $11.6 \mathrm{~mm}(\mathrm{P} 25=7 \mathrm{~mm}, \mathrm{P} 75=17 \mathrm{~mm})$ for successful cases and $-4 \mathrm{~mm}(\mathrm{P} 25=-6.5 \mathrm{~mm}, \mathrm{P} 75=1.5 \mathrm{~mm})$ for failed cases $(\mathrm{P}=0.002)$.

Conclusion: Careful preoperative anatomical assessment of the LCIV on the MRI may improve patient selection for this novel technique.

203 Normative Study of the Intervertebral Disk Measurement in Young Healthy Adults

Saman Shabani, BS, MD; Arash Razmjoo, PhD; Nicholas DeVogel; Yoganadan Narayan, PhD; Jamie Baisden, MD

Introduction: Adjacent segment disease commonly develops following cervical fusion. Finite element method (FEM) is a form 
of mathematical model that simulates various loading conditions to predict the biomechanical response of the spine. However, current FEM do not take into account different intravertebral disc height and specific region differences which makes it less specific. This normative study can help to better define the disc measurements in the cervical spine and help to create a more accurate FEM.

Methods: A retrospective analysis of 36 young adult (18-41 y/o) healthy volunteers who underwent cervical spine quantitative computed tomography (CT) is done. The IVD height was measured at anterior, middle, and posterior aspect of the disc at $\mathrm{C} 2 / 3$ to $\mathrm{C6} / 7$. A linear mixed model was constructed to account for the repeated measures on each patient, with fixed effects for disc, measurement, an interaction between the two, and a random effect for patient. All pairwise comparisons between discs and all pairwise comparisons between measurement types were constructed. Holm's adjustment was made to the p-values.

Results: All measures were significantly different from each other with adjusted p-values $<0.001$ (Posterior Middle: 1.4, Anterior Posterior: 0.38, Middle Anterior: 1.04). There was only a significant different between discs C6/7 C2/3 (0.41, adjusted $\mathrm{p}=0.0432)$ and discs C5/6 C2/3 (0.26, p <0.001). The average height of $\mathrm{C} 6 / 7$ is greater than others by an expected amount of 0.2698 with adjusted $p$ of 0.0024 .

Conclusion: This is a normative data which has never been reported in literature from a group of healthy and young volunteers with normal bone mineral density (BMD). This data can be used to model healthy spines as level and disc region specific for analysis of mathematical models such incorporation in the finite element studies.

204 Economic Performance of Oblique Lateral Lumbar Interbody Fusion (OLLIF) with a Focus on Hospital Throughput Efficiency

\section{Hamid Reza Abbasi, MD, PhD}

Introduction: Between 1998 and 2008 the number of spinal fusions in the U.S. increased 2.4 times and the cost per fusion increased 3.3 times, leading to a 7.9 fold increase in the cost burden of spinal fusions to the U.S. healthcare system. OLLIF is a new minimally invasive procedure for fusions of the Lumbar spine that can be employed safely and effectively from T12-L1 to L5-S1. OLLIF approaches the disk space through Kambin's triangle. OLLIF does not require direct visualization but instead relies on bilateral fluoroscopic imaging and electrophysiological monitoring. OLLIF has reduced surgery times and hospital stay compared to TLIF. The purpose of this study is to evaluate the preoperative cost of OLLIF compared to TLIF.

Methods: The study population is comprised of 69 OLLIF patients and 58 open TLIF controls. All patients underwent the full course of conservative therapy. Indications were Degenerative Disk Disease, Disk Herniation, Listhesis, Stenosis (except Osteogenic Stenosis). This is a retrospective cohort study. All surgeries were single surgeon procedures and all TLIF cases were completed before the surgeon started performing OLLIF. We recorded surgery time, length of stay and infection rates. Perioperative outcomes were monetized by using a multiplier approach. OR Time was monetized at $\$ 83.51 /$ minute and hospital stay at $\$ 2197 /$ day. Infection rate was monetized relative to a $2.4 \%$ infection rate for open procedures.

Results: OLLIF cuts surgery times and hospital stay in half relative to TLIF (59/132 $\mathrm{min}, 4.7 / 2.3$ days respectively). When these differences are monetized, OLLIF reduces the average cost per surgery relative to TLIF by $\$ 11,834$ per surgery, with higher cost reductions for multi-level procedures. In over 500 OLLIF procedures to date, there have been no deep infections requiring drainage and only a single superficial infection $(0.2 \%)$. We estimate that the reduction in infections saves an additional $\$ 316$ per surgery, for a total cost reduction of $\$ 12,150$.

Conclusion: OLLIF is a new MIS spinal fusion that reduces perioperative costs relative to open surgery and could potentially reduce U.S. health care expenditures by $\$ 2.5$ billion per year, through the preoperative cost savings alone. A comprehensive cost analysis is underway.

205 Minimally Invasive Far Lateral Lumbar Microdiscectomy with Intraoperative Imaging and Neuronavigation

David B Choi, MD; Hesham M. Soliman, MD; Jared Fridley; Michael Galgano, MD; Thomas Kosztowski, BS; Albert Edward Telfeian, MD, PhD; Ziya L. Gokaslan, MD; Adetokunbo A. Oyelese, MD, $\mathrm{PhD}$

Introduction: Far lateral disc herniations comprise up to $12 \%$ of all lumbar disc herniations. Advantages of a far lateral approach include more direct access to the disc and less disruption of osteo-ligamentous structures. Intraoperative imaging, coupled with neuronavigation, adds precision and safety to this procedure.

Methods: Patient 1 was a 35-year-old female presenting with 3 days of right dorsiflexion weakness, rated 4/5, along with hypoesthesia of the dorsum of the right foot. Preoperative MRI demonstrated a far lateral disc herniation at L5-S1. Patient 2 was a 51-year-old male presenting with a right-sided L3-4 far lateral disc herniation resulting in severe pain in a right $\mathrm{L} 3$ distribution, refractory to conservative measures. Both patients were positioned prone on a radiolucent operating table, and prior to skin incision, a digital reference array was secured with Schanz pins to the iliac crest. A single intraoperative CT was obtained to plan the incision above the facet joint. After dissection through the intertransverse ligament, neuronavigation aided in locating the super articular process of the caudal level. A minimally-invasive burr was used to remove the lateral aspect of the superior articular process, thereby exposing the disc space. Neuronavigation ensured precise removal of the lateral facet without destabilizing the joint. Postoperatively, Patient 1 experienced improvement in sensation along the dorsal right foot, as well as dorsiflexion strength postoperatively. Patient 2 experienced improvement in his right L3 radiculopathy, rated $5 / 10$.

Results: The average patient age was 43 years. Average operating time was 144 minutes, and estimated blood loss ranged from 5 to $25 \mathrm{cc}$. One CT scan was performed in each case, with an average total computed tomography dose index (CTDI vol) of 34.61 MGy.

Conclusion: Intraoperative imaging with neuronavigation is a powerful tool for far lateral lumbar discectomies. The surgeon can safely and precisely perform decompression without compromising the nerve root and mechanical stability.

206 Minimally Invasive Transforaminal Lumbar Interbody Fusion Using a Novel Autograft Bone Collector is a CostSaving Alternative Method for Spinal Fusion Surgery

Mick J. Perez-Cruet, MD, MS; Esam A Elkhatib, MD, PhD; Katie Mansour; Ratnesh Mehra, D.O.

Introduction: Lumbar spinal fusion surgery is preformed to alleviated debilitating back pain symptoms due to a variety of spinal conditions including spondylolisthesis with or without spinal stenosis. Sources of bone graft can include autograph, allograft or bone substitutes. The BoneBac Press (Thompson MIS, Salem, $\mathrm{NH}$ ) was developed to collect drilled local bone collected during the decompression and or approach. This study analyzed the 
cost savings and effectiveness of collecting local bone, need for additional allograft, and fusion rates while preforming minimally invasive transforaminal lumbar interbody fusion (MITLIF).

Methods: A retrospective analysis was preformed of 93 patients who received MITLIF (using Thompson MIS TLIF system). One level MITLIF was preformed using the system in $95 \%$ of patients. The amount of local autography collected using the BoneBac Press was recorded. The amount of additional allograph supplement was also recorded for each case and the ratio of autograph to allograph used. The cost of each surgery was estimated based on the allograph savings.

Results: The average autograft volume collected was $8.01 \mathrm{cc} /$ case, with a total of $753 \mathrm{cc}$ collected over the 93 patients analyzed. The percentage of autograft to allograft used ranged from $42 \%$ $333 \%$ with the vast majority of cases using $2 / 3$ autograph to $1 / 3$ allograph. Cost savings range from $\$ 1820.00$ to $\$ 6145.50$ per case with an average $\$ 4965.43 /$ case cost savings. The estimated total savings for this series of patients was $\$ 342,615.00$ (Fig. 9). A greater than $95 \%$ fusion rate was achieved based on dynamic radiographic assessment at 3,6 , and 12 month post-operative intervals. No patient returned to OR for revision surgery at the MITLIF level.

Conclusion: The use of BoneBac Press harvested morselized autografts with or without supplemental allografts to achieve MITLIF is an extremely cost effective method of achieving high rates of spinal fusion. Further multi-center prospectively randomized trials might provide additional cost benefits and improvements in patient generated outcomes.

207 Anterior Lumbar, Oblique Lateral, and Transforaminal Lumbar Interbody Fusions: A Comparison of Perioperative Complications

Caleb S Edwards, BA; Anthony Michael DiGiorgio, DO, MHA; Darryl Lau, MD; Praveen V. Mummaneni, MD; Dean Chou, MD

Introduction: Interbody fusions can be performed via anterior lumbar (ALIF), oblique lateral (OLIF), and transforaminal (TLIF) approaches. We sought to compare complication rates of these modalities.

Methods: Retrospective review of ALIF, OLIF, and TLIF cohorts was undertaken. Demographics, ASA scores, operative time, estimated blood loss, infection, intraoperative CSF Leak, vascular injury, post-operative ileus, motor deficits and 90-day readmissions were evaluated.

Results: 213 patients were included: 90 ALIF patients, 49 OLIF patients, 74 TLIF patients. OLIF patients were older than ALIF and TLIF (67y vs 61y and 61y; $\mathrm{p}<0.05)$. ALIF cohort $(52 \%$ male; $67 \%$ prior surgery) had larger percentage of males and prior spinal surgeries than OLIF $(37 \% ; 59 \%)$ and TLIF $(36 \% ; 54 \%)$. No significant differences were observed in ASA scores and procedure time. OLIF cases had significantly less blood loss compared to ALIF and TLIF $(229 \mathrm{ml}$ vs $814 \mathrm{ml}$ and $578 \mathrm{ml}$; $\mathrm{p}<0.05)$. Nearidentical infection rates were seen for ALIF, TLIF, and OLIF ( $4 \%$ vs $3 \%$ vs $2 \%$; $>0.05)$. TLIF had highest risk for durotomy $(3 \%)$, followed by ALIF (1\%) and OLIF $(0 \%)$. Vascular injury was highest for ALIF (8\%) compared to OLIF (2\%) and TLIF (0\%). Postoperative ileus was highest in ALIF patients (20\%), compared to OLIF $(6 \%)$ and TLIF (3\%). At most recent follow-up, ALIF $(19 \%)$ and TLIF (15\%) had more motor deficits than OLIF $(6 \%)$. OLIF (4\%) had marginally lower rates of 90-day surgery related readmissions than TLIF $(8 \%)$ or ALIF $(7 \%)$.

Conclusion: Within our series, OLIF had the lowest complication rate when compared to ALIF and TLIF. This may be because of the minimally invasive approach, avoidance of direct neural manipulation either posteriorly or through the lumbar plexus, and operating through a corridor without needing to mobilize the great vessels.

208 Propensity Matched Comparison of Outcomes and Cost After Macroscopic and Microscopic Lumbar Discectomy Using a National Longitudinal Database

Allen L Ho, MD; Arjun Vivek Pendharkar, MD; Paymon Rezaii; Eric S Sussman, MD; David Arnold Purger, MD; Anand Veeravagu, MD; John K. Ratliff, MD, FACS; Atman Desai, MD, MA

Introduction: There has been considerable debate about the utility of the operating microscope in lumbar discectomy and its effect on outcomes and cost.

Methods: A commercially available longitudinal database (MarketScan) was used to identify patients undergoing lumbar discectomy with or without microscope between 2007-2015. Propensity matching was performed to normalize differences between demographics and comorbidities in the two cohorts. Outcomes, complications and cost were subsequently analyzed with multivariate logistic regression.

Results: A total of 42,025 patients were identified for the 'macroscopic' group, while 11,172 patients were identified for the microscope group. There were no significant differences in postoperative complications between the groups other than a higher proportion of deep vein thrombosis (DVT) in the macroscopic cohort $(0.4 \%)$ versus the microscopic discectomy group $(0.4 \%$ vs $0.2 \%$; matched OR $0.48(0.26-0.82) ; p=0.0045)$. Length of stay was significantly longer in the macroscopic group compared to microscopic discectomy (2.13 days vs 1.83 days $\mathrm{p}<0.0001)$. Macroscopic discectomy patients had a higher rate of revision surgery when compared to microscopic discectomy (OR 0.92 $(0.84-1.00) ; \mathrm{p}=0.0366)$. Hospital charges were higher in the macroscopic group ( $\$ 19,490$ vs $\$ 14,921 ; \mathrm{p}<0.0001)$.

Conclusion: The present study suggests that the use of the operating microscope in lumbar discectomy is associated with decreased length of stay, lower DVT rate, reoperation rate and decreased overall hospital costs.

209 Assessment of Individual Radiation Dose to Operating Room Staff During Minimally Invasive Spinal Surgery: Prospective Series Using a Personal Device

Jakub Godzik, MD, MSc; Gautam Nayar; S. Harrison Farber, MD; William D. Hunter, MD; Luis Manuel Tumialan, MD

Introduction: Minimally invasive approaches decrease the cost and morbidity of spinal surgery, but require additional intraoperative imaging for anatomical visualization. Conventional fluoroscopy remains the most common imaging option, but exposes all operating room staff to increased radiation. The objective of the current study was to compare radiation exposure between operating room personnel during transpsoas lumbar interbody fusion (LLIF) or minimally invasive transforaminal lumbar interbody fusion (MIS-TLIF).

Methods: Operating room staff participating in 50 MIS surgery procedures under a single surgeon were prospectively enrolled in this study. All radiation dose exposures were recorded for each member of the surgical team (surgeon, assistant, scrub, circulator) using DoseAware, a personal radiation dose management device. Radiation doses were recorded in milliseverts $(\mathrm{mSv})$. Radiation exposure by the c-arm was collected from the fluoroscope, recorded in milligray ( $\mathrm{mGy}$ ). Comparisons between groups were made using ANOVA, with Tukey's post hoc test.

Results: Twenty-five patients underwent LLIF, of whom 11 
underwent posterior percutaneous screw placement (PSP), and 14 underwent MIS-TLIF. Throughout the three procedures, the surgeon experienced the highest average radiation exposure (LLIF: 159.5, PSP: 275.5, MIS-TLIF: $397.9 \mathrm{mSv}$ per level, $\mathrm{p}<0.01)$ followed by the assistant (LLIF: 130.6, PSP: 136.0, MIS-TLIF: 274.9 $\mathrm{mSv}$ per level, $\mathrm{p}=0.013$ ). The circulator (LLIF: 2.0, PSP: 6.2, MIS-TLIF: $10.2 .3 \mathrm{mSv}$ per level, $\mathrm{p}<0.01)$ and the scrub nurse had significantly lower exposure (LLIF: 40.3, PSP: 156.8, MIS-TLIF: $280.7 \mathrm{mSv}, \mathrm{p}<0.01)$ All OR personnel recorded significantly lower radiation with LLIF compared to MIS-TLIF $\mathrm{p}<0.029)$. Radiation exposure was not correlated with BMI ( $>00.233)$, however it was positively correlated with age $(\mathrm{p}<0.004)$.

Conclusion: All OR personnel are exposed to significant radiation doses during minimally invasive surgery, with surgeon and assistant having the greatest exposure. Additionally, staff members undergoing LLIF had significantly less radiation exposure than during MIS-TLIF.

210 Mini-open Lateral En Bloc Corpectomy: Cadaveric Feasibility and Early Clinical Experience

Jay D. Turner, MD, PhD; Hasan A Zaidi, MD; Jakub Godzik, MD, MSc; Felipe Albuquerque, MD; Juan S. Uribe, MD, FAANS

Introduction: Traditional open approaches to the thoracolumbar spine for en bloc resection of vertebral body tumors are associated with significant surgical morbidity. Less invasive surgical techniques have the potential to reduce complications.

Methods: The feasibility and safety of the technique were evaluated in 3 fresh cadaveric specimens. In each specimen, the procedure was performed at 3 separate levels (L1-L3), 9 levels total. A staged posterior midline and extracoelomic mini-open anterolateral approach for en bloc corpectomy was performed and the neurovascular structures were evaluated for tissue injury. These techniques were then applied to a clinical case for resection of a lumbar epithelioid hemangioendothelioma. The operative experience, complications, and early surgical and clinical outcomes are reviewed.

Results: In the cadaveric study, the combined approach allowed for en bloc corpectomy with no appreciable injury to critical neurovascular structures. The procedure was reproduced in the clinical setting, with early results demonstrating favorable clinical and radiographic outcomes.

Conclusion: Our early experience suggests that a staged posterior and mini-open anterolateral extracoelomic approach allows for safe and effective en bloc resection of vertebral body tumors. This approach may limit the surgical morbidity associated with traditional open techniques. The use of this new technique in large case series and long-term follow-up to determine outcomes are needed for better understanding of the strengths and limitations of the technique.

211 Success and Failure of Minimally Invasive Direct Pars Repair: Does Fracture Morphology Matter?

Islam Fayed, MD, MS; Anthony G Conte, MD; Jean-Marc Voyadzis, MD

Introduction: Spondylolysis is a defect in the pars interarticularis that typically presents with axial back pain. Recently, minimally invasive spine techniques have gained popularity and have been applied to Buck's technique of direct pars repair.

Methods: Two patients underwent minimally invasive direct pars repair by the percutaneous insertion of a cannulated lag screw across the pars defect with compression against the lamina. The defect was then decorticated and packed with bone grafting material through a tubular retractor. Clinical records including, preoperative imaging characteristics and intraoperative variables, were collected.

Results: Two patients presented with pars defects at L5 causing low-back pain refractory to conservative management. The first was a 22-year-old female with bilateral pars defects, and the second was a 21-year-old female with a left-sided pars defect. They underwent minimally invasive direct pars repair without complication and were discharged home within $24-48$ hours. The first patient fused successfully, while the second experienced screw backout requiring subsequent revision. Follow up time was 25 months and 21 months, respectively. Demographic data was similar, including age, gender, weight, activity level, and use of a brace. The first patient was mobilized at two weeks with physical therapy, while the second patient was mobilized at six weeks. Fracture morphology also differed: the successfully repaired fractures were bilateral, linear fractures with smooth cortical edges, while the failed repair involved a unilateral, curved defect with comminuted cortical edges.

Conclusion: Minimally invasive direct pars repair can be performed safely and effectively with shortened hospital stays and reduced morbidity. Fracture morphology and orientation may be an important predictor of the success of surgery.

212 Endoscopic Intralaminar Lumbar Microdiscectomy Technique

\section{Nathaniel P. Brooks, MD}

Introduction: The lumbar microdiscectomy is a commonly performed surgical procedure. Recent advancements in endoscopic tools have made it possible to perform an intralaminar microdiscectomy through a single incision. The purpose of this study is to demonstrate the technique of the endoscopic intralaminar discectomy using intraoperative video.

Methods: The patient is positioned in a prone position. Fluoroscopy is used to localize the posterior lumbar surgical incision overlying the intralaminar window at the desired lumbar level. A $10 \mathrm{~mm}$ skin incision is then made and a tissue dilator is advanced to the level of the ligamentum flavum under fluoroscopic guidance. A $7 \mathrm{~mm}$ beveled working cannula is then advanced over the tissue dilator. The endoscope is then inserted and visualization is enhanced using gentle saline irrigation. Bipolar cautery is used to coagulate the muscle fibers remaining over the ligmentum flavum. The ligament is then thinned using a tissue punch. The working cannula is then advanced through the ligamentum under direct endoscopic visualization. The traversing nerve root is then mobilized and medialized using dissectors and the beveled cannula. The epidural veins are cauterized, the annulus incised and free disc fragments removed to decompress the nerve. Decompression of the nerve can be confirmed with visual and tactile evaluation.

Results: The technique for the endoscopic intralaminar microdiscectomy is demonstrated using video.

Conclusion: The endoscopic intralaminar microdiscectomy is a technically feasible option for resection of lumbar disc herniations. Prospective cohort studies have demonstrated that this technique has equivalent outcomes to open microdiscectomy with moderately faster recovery. 1

213 Minimally Invasive Anterior and Lateral Transpsoas Approaches for Closed Reduction of Grade II Spondylolisthesis: Initial Clinical and Radiographic Experience FAANS

David S. Xu, MD; Konrad Bach, MD; Juan S. Uribe, MD,

Introduction: Minimally invasive anterior and lateral 
approaches to the lumbar spine are increasingly used to treat and reduce Grade I spondylolisthesis, but concerns still exist for their usage in the management of higher grade lesions. We report our experience using this strategy for Grade II spondylolisthesis in a single surgeon case series and provide early clinical and radiographic outcomes.

Methods: A retrospective review of a single surgeon's volume between 2012 and 2016 identified all patients with a lumbar spondylolisthesis of Meyerding Grade II that underwent a minimally invasive lateral lumbar interbody fusion (LLIF) or anterior lumbar interbody fusion (ALIF) targeting the slipped level, without posterior direct decompression. Demographic, clinical, and radiographic data were collected and analyzed. Statistical testing was performed using the paired t-test and Wilcoxon signed ranks test for continuous and ordinal variables respectively.

Results: Twenty-four patients were included in our study. The mean follow-up time for LLIF and ALIF patients were 17.6 (SD 12.5) months and 10 (SD 3.1) months respectively. Complete reduction of the spondylolisthesis was achieved in $13(81.3 \%)$ LLIF patients and 7 (87.5\%) ALIF patients. Across both procedures, there was an increase in both the segmental lordosis (LLIF 5.3 degrees, $\mathrm{p}$-value $=0.002$; ALIF 15.0 degrees, $\mathrm{p}$-value $=0.002$ ) and overall lumbar lordosis (LLIF 2.9 degrees, p-value 0.151; ALIF 5.1 degrees, p-value $=0.006$ ). A statistically significant decrease in the mean VAS and ODI measurements were seen in both treatment groups (LLIF, VAS 3.9 p-value $=0.002$, ODI 19.8, p-value $=0.001 ;$ ALIF, VAS 3.8, p-value, ODI 21, p-value $=0.03$ )

Conclusion: Early clinical and radiographic results from using minimally invasive LLIF and ALIF approaches to treat Grade II spondylolisthesis appear to be good with low operative blood loss and no persistet neurologic deficits. Complete reduction of the spondylolisthesis is frequently possible with statistically significant reduction in pain scores.

214 Percutaneous Transforaminal Interbody Fusion with Expandable Mesh Bag System Preliminary Results

Kevin T. Huang, MD; Muhammad M Abd-El-Barr, MD, PhD; Yi Lu, MD, PhD; John H. Chi, MD, MPH

Introduction: With the advent of minimally invasive surgery (MIS) techniques for lumbar spine surgery, there has been an increased interest in methods to accomplish interbody fusion with minimal muscle dissection, bony drilling and nerve retraction, yet provide biomechanical stability. In this study, we review our preliminary experience using an expandable mesh bag system that is used as an interbody spacer that can be placed percutaneously in patients undergoing transforaminal interbody fusion (TLIF) procedures.

Methods: A retrospective review of our first 14 cases of percutaneous TLIF were reviewed. Demographic data were reviewed. Intraoperative records were reviewed for complications and blood loss. Clinical records were reviewed to assess how patients were doing after surgery.

Results: The average age of the patients at surgery was 53 years with a standard deviation of 11 years. Six out of fourteen (43\%) of patients were female. Seven out of 14 patients $(50 \%)$ of patients underwent L4-5 TLIF, 5 (36\%) underwent L5-S1 TLIF, one underwent L3-4 TLIF and another one underwent L4-S1 TLIF. No intraoperative complications were noted. Average blood loss was approximately $25 \mathrm{~mL}$. The average clinical and imaging follow up time was 1 year. Nine of $14(65 \%)$ of patients had an excellent result after surgery. Only 2 patients (14\%) did not have a positive result after surgery. One patient developed a herniated disc at an adjacent level that was treated conservatively. One patient showed evidence of subsidence of cage on postoperative imaging.
Conclusion: Percutaneous placement of an expandable mesh bag system for interbody fusion appears to be a safe and effective method. The decreased blood loss and morbidity due to minimizing muscle dissection, bony drilling and nerve root manipulation are positive characteristics of this system.

215 Clinical Outcomes Following MIS vs. Endoscopic Laminectomy

Lynn B McGrath, MD; Gabrielle A White-Dzuro, MD; Christoph Paul Hofstetter, MD, PhD

Introduction: Minimally invasive spine (MIS) surgery for laminectomy has gradually gained acceptance as a safe and efficacious counterpart to traditional open techniques. Given that it spares midline structures and facet joints it has been proposed to cause less postsurgical segmental instability. We have further advanced this technique utilizing the interlaminar endoscopic technique. The endoscope allows for high resolution off-axis visualization of neural structures within the lateral recess thereby minimizing the need for facet joint resection. The efficacy and safety of MIS vs. endoscopic techniques have not been studied.

Methods: A retrospective analysis of 96 consecutive patients undergoing either MIS $(n=49)$ or endoscopic $(n=47)$ laminectomy for spinal stenosis was performed. Patient demographics, operative details, clinical outcome, and complications were reviewed.

Results: Our patient cohort consisted of 41 females and 55 males with an average age of 62 years. Half of the patients had single-level, a third 2-level and the remaining patients 3- or 4-level procedures. Surgical times were significantly longer for endoscopic surgeries compared to MIS (204 min vs. $162 \mathrm{~min}$ ). Surgeries were generally well tolerated. Two patients in the MIS group had durotomies. Hospital stay for MIS patients was on average 2.4 days compared to 0.7 days for endoscopic patients. At one-year follow-up endoscopic patients had a trend towards less back pain compared to MIS patients (VAS: 3.3 vs. 4.6). Endoscopic laminectomy patients had significantly less leg pain (VAS: 1.6 vs. $3.4 ; \mathrm{P}<$ $0.05)$ and less disability (ODI: 23.7 vs. $37.7 ; \mathrm{P}<0.05$ ).

Conclusion: Endoscopic laminectomy is a safe and effective procedure with favorable complication profile and patient outcome.

216 Cost Comparison of Standalone Lateral Lumbar Interbody Fusion

Andrew M Faramand, MD; Nitin Agarwal, MD; Nima Alan, MD; David Salvetti, MD; Naveed Ismail, B.A.; D. Kojo Hamilton, MD; David O. Okonkwo, MD, PhD; Adam S. Kanter, MD

Introduction: Lateral Lumbar Interbody Fusion (LLIF) is a minimally invasive procedure that is used to treat a variety of spine disorders. With the continuously rising healthcare costs, we sought to perform a direct cost comparison of LLIF between elderly $(=65)$ and younger $(<65)$ patients.

Methods: Financial data of 116 patients who underwent standalone LLIF between July 2013 and January 2017 were analyzed. The preoperative and postoperative values for the Oswetry Disability Index (ODI) were also analyzed to compare outcomes. Total costs were based on the length of hospital stay, anesthesia, operative, laboratory, imaging, and administrative costs. Correlation among age, number of levels fused, and length of hospital stay was performed.

Results: Seventy-six patients were under the age of 65 and 40 patients 65 years or older who underwent standalone LLIF were included in this analysis. Postoperatively, $85 \%$ of patients over the age of 65 years had improved ODI score, compared to 
$84 \%$ of the younger cohort $(\mathrm{p}=0.59$ ). The mean cost in elderly of $\$ 20,421$ was significantly higher than the mean cost of the young, $\$ 17,099(\mathrm{p}=0.022)$. Patients over the age of 65 years had a longer length hospital stay of 2.18 days, compared to 1.5 days in younger patients $(\mathrm{p}=0.027)$; this was associated with higher costs $(p=0.001)$. Matching patients based on the number of levels fused demonstrated that laboratory $(\mathrm{p}=0.001)$, imaging $(\mathrm{p}=0.001)$, administrative $(\mathrm{p}=0.02)$, and anesthesia $(\mathrm{p}=0.003)$ expenses where significantly higher for elderly patients. The number of levels fused was significantly higher in the elderly patient group $(p=0.0001)$.

Conclusion: While costs associated with a LLIF were higher in patients over the age of 65 , from this analysis, we conclude that these increased expenses incurred by the elderly group were mainly attributed to non-operative factors.

217 Assessment of Radiographic and Clinical Outcomes of an Articulating Expandable Interbody Cage in Minimally Invasive Transforaminal Lumbar Interbody Fusion For Spondylisthesis

Lara Walsh Massie, MD; Hesham Mostafa Zakaria, MD; Azam Basheer, MD; Lonni Schultz, PhD; Morenikeji Buraimoh, MD; Victor W. Chang, MD

Introduction: Inability to correct sagittal imbalances and risk of subsidence have been a limitation of traditional bullet shaped minimally-invasive transforaminal interbody fusion (MISTLIF) devices. This study evaluates the use of a crescent shaped articulating expandable lordotic cage, Altera (Globus Medical) in MIS-TLIF.

Methods: A retrospective review 44 consecutive patients treated at 49 surgical levels. Radiographic outcomes included segmental and global lordosis (L1 to S1), disc height, presence of interbody fusion, and any endplate violations. Clinical outcomes included Numeric Rating Scale for leg and back pain (NRS), and Oswestry Disability Index for low back pain (ODI).

Results: Thirty-nine patients underwent single level MIS-TLIF, and 5 underwent two level MIS-TLIF. Median OR time for the single level procedures was 139 minutes. On average, spondylolisthesis corrected $4.3 \mathrm{~mm}$ (pre $=6.69 \mathrm{~mm}$, post $=2.39 \mathrm{~mm}, \mathrm{p}<0.001$ ), segmental angle improved $4.94^{\circ}\left(\right.$ pre $=5.63^{\circ}$, post $\left.=10.58^{\circ}, \mathrm{p}<0.001\right)$, and segmental height increased $3.1 \mathrm{~mm}$ (pre $=5.09$, post $=8.19$, $\mathrm{p}<0.001)$. Sagittal parameters trended toward improvement. Thirtynine of the 40 levels (92\%) with one year CT data had solid fusion across the disc space, through the interbody at one year. Fourteen patients had suspected endplate violation during placement of the cage. Only three of these had any progression of subsidence with an overall subsidence rate of $6 \%$ (3 of 49), none of which required further intervention to date. At 90 days, there were significant reductions in back pain and leg pain on the NRS of -4.2 points $(\mathrm{p}<0.001)$, and -4.3 points $(\mathrm{p}<0.001)$ respectively. Mean ODI was reduced by 17.6 points, from 41.6 to $27.4(\mathrm{p}<0.001)$.

Conclusion: The use of this expandable, articulating, lordotic interbody cage for MIS-TLIF provides a significant restoration of spondylolisthesis, segmental height and lordosis, with trends toward improvement in sagittal balance parameters. Patients treated with this technique had acceptable levels of fusion and significant reductions in pain and disability.

218 Comparison of Lordosis Correction with Transforaminal Lumbar Interbody Fusion Via Open or Minimally Invasive Approach

Logan Helland, MD; Royce Woodroffe, MD

Introduction: Lumbar spondylosis is a significant public health problem and is associated with significant disability and pain for those affected. A current treatment modality includes transforaminal lumber interbody fusion (TLIF) via a traditional open or minimally invasive (MIS) approach. Outcomes between the two procedures were reviewed to examine if there is a benefit when comparing approaches.

Methods: A retrospective review was performed of patients over the last 10 years undergoing open or MIS TLIF by a single surgeon. Post-operative and pre-operative lumbar spine imaging was reviewed for lumbar lordosis and segmental lordosis angles. We also examined operative time, blood loss, hospital stay, and operative cost as well as, Visual-analog (VA) pain scale and Oswestry Disability Index (ODI).

Results: 89 patients (26 MIS, 63 open) were reviewed from a single surgeon experience. Open surgery patients had an increased likelihood to have more posterolateral fusion levels and interbody levels fused than MIS fusions. Estimated blood loss was significantly less during surgery for the MIS group, but there was no difference in transfusion rate as a result. No differences were observed for lumbar or local lordosis before and after surgery for each method. There were no differences in Pre-operative, postoperative, or overall improvement of VA pain scale or ODI. No differences were observed for rate of reoperation for infection, hardware failure, or adjacent level disease as well. Cost was significantly more for open procedures.

Conclusion: There is no significant benefit to MIS or open TLIF for the correction of lumbar lordosis. While open surgery did have more blood loss compared to MIS, open surgery on average involved more levels. Open surgery was more expensive than MIS, but can also be explained by open surgeries including more levels on average. There was no advantage to improving disability or pain when comparing each surgery type to each other.

219 Safety and Efficacy of Outpatient Minimally Invasive Decompression Surgeries on the Thoracic Spine Via LED or LFD

Stephen Songhurst; Michael Weiss, MD; Chip Wade, PhD; Reginald J. Davis, MD, FAANS, FACS

Introduction: Spinal stenosis of the thoracic spine is uncommon. The most frequently identified clinical syndrome being associated with thoracic spinal canal stenosis is a degenerative disorder of the spine [1]. Patient-reported outcomes and surgical variables for patients undergoing minimally invasive surgeries (MIS) on the thoracic spine in a free-standing ambulatory surgery center (ASC) are not well-documented. This study examines changes in pain and disability for patients undergoing outpatient thoracic MIS.

Methods: From September 2012 to January 2017, 114 patients (50.57 years [range 24-73 years]) underwent a thoracic MIS. Of that total, 85 patients underwent a lateral extraforaminal decompression (LED) procedure, while 29 patients underwent a laminotomy foraminotomy decompression (LFD). Estimated blood loss (EBL), intraoperative complications, length of surgery (LOS), return to work (RTW), visual analog scale (VAS) and Oswestry disability index (ODI) were collected preoperatively and up to 36 months postoperatively.

Results: Significant reductions were reported for the VAS (6.35 to 3.69) and ODI (44.49 to 27.25) from preoperative to postoperative. The average EBL and LOS were $39.30 \mathrm{~mL}$ and 47.70 minutes, respectively. The ?VAS for the LFD and LED cohorts was 2.76 versus 2.63 , respectively. The ?ODI in the LFD and LED cohorts was 18.21 versus 16.92 , respectively. The average EBL in the LED and LFD cohorts was $27.59 \mathrm{~mL}$ versus $73.62 \mathrm{~mL}$, respectively. The average LOS in the LED and LFD cohorts was $45 \mathrm{~min}$ versus $55.62 \mathrm{~min}$, respectively. A total of 89 patients reported 
RTW data, and $82(92.13 \%)$ returned to work by the postoperative interval. One intraoperative complication was reported.

Conclusion: Our study suggests that MIS-LFD and MIS-LED procedures on the thoracic spine can be performed safely and effectively in a free-standing ASC. Results indicate that patients undergoing MIS-LFD will experience greater improvements in pain and disability from preoperative to postoperative than patients who undergo MIS-LED.

220 Assessment of Clinical Outcomes and Efficacy of Minimally Invasive Transforaminal Lumbar Interbody Fusion (TLIF) in Obese Patients with Debilitating Back Pain from Spondylolisthesis.

Mick J. Perez-Cruet, MD, MS; Esam A Elkhatib, MD, PhD; Elizabeth Abel, BS

Introduction: Obesity is a major health problem in patients suffering from debilitating back pain that contributes to postoperative complications. Minimally invasive treatment (TLIF) can provide benefits by proving smaller incisions, reduced tissue destruction, less post operative pain, early ambulation, and less hospital stay.

Methods: Eighty-five patients (55(65\%) female, 30(35\%) male, average age 65 years old) with BMI greater than $30 \mathrm{~kg} /$ $\mathrm{m} 2$ with grade I or II spondylolisthesis operated by TLIF were retrospectively reviewed between November 2011 to April 2017. These patients were categorized into three obesity categories: BMI $30.134 .9 \mathrm{~kg} / \mathrm{m} 2$ (Class I), $35.039 .9 \mathrm{~kg} / \mathrm{m} 2$ (Class II), and $=40.0$ $\mathrm{kg} / \mathrm{m} 2$ (Class III). Oswestry disability index, Pain analogue scale, operative time, estimated blood loss (EBL), post-operative complications and hospital stay (LOS) and fusion rates were analyzed.

Results: Average follow-up time was 2.2 years. Obesity categories included the following: 40 class 1, 29 class 2, and 16 class 3 having chronic back pain symptoms averaging 5 years range 1 to 30 years. All patients underwent single level TLIF at the L3-4(n=6 ), L4-5(n= 58), or L5-S1 $(n=21)$ level. Mean operative time was 199 minutes +/- 45 minutes, EBL was $144 \mathrm{ml} \mathrm{+/-} 55 \mathrm{ml}$ and mean LOS was 4.4 days +/- 2.1 days. VAS decreased from 6.55 pre-op to 2.8 at 3 months postoperative and 3.4 at one year, ODI decreased from 44.7 pre-op to 27.7 at 3 months post-op and 30.5 at one year. Complications rate was $4.9 \%$, fusion rate was $>93 \%$ at 3 and 12 months follow-up based on dynamic plain radiographs. Re-operation rates was $1.1 \%$ for adjacent level pathology.

Conclusion: Obese patients with debilitating back pain from lumbar spondylolisthesis can be safely and effectively managed with minimally invasive TLIF to reduce complications, improve fusion rates, and provide excellent clinical outcomes.

221 Utilization Trends of Cervical Disk Replacement in the United States: A Population-Based Study

Panagiotis Kerezoudis; Mohammed Ali Alvi, M.D.; Anshit Goyal, MBBS; Daniel S Ubl, BA; Sandy Goncalves, MSc; Jenna Meyer; Elizabeth B Habermann, PhD; Mohamad Bydon, MD

Introduction: Cervical disc replacement(CDR) has emerged as a safe and viable surgical technique for the management of patients with cervical disc disease. The current consensus on the indications for CDR are patients with single-level disc disease with primarily anterior or disc-related pathology without significant facet joint arthropathy and preserved segmental motion, disc space height and sagittal alignment. This study aimed to characterize the utilization trends of CDR in the United States including off-label use in a national, administrative database.

Methods: The National Inpatient Sample (NIS) was queried for the years 2004 through 2013 for patients undergoing CDR. Off-label utilization comprised the primary outcome and was defined by(i)the presence of concurrent cervical fusion(ii)performance in the emergent setting(iii)surgical diagnosis outside of approved indications. We also recorded cases where the procedure was performed in the presence of a known contraindication. National estimates were calculated using the discharge weights for each sampled hospital.

Results: A total of 22,232 CDR cases (90\% elective) were performed in the U.S. between 2004 and 2013. Seventy one percent of elective cases were stand-alone CDRs, while $29.3 \%$ were done in conjunction with a cervical fusion procedure ( $94.5 \%$ anterior fusion, $2.3 \%$ posterior, $3.1 \%$ both). Within emergent cases, $66.4 \%$ were stand-alone and $33.6 \%$ were done in conjunction with a fusion procedure. Overall, we observed a significant increase in CDR utilization with or without concurrent fusion between 2004/2005 and 2009 followed by a steady decline. Several cases of CDR were performed in the presence of known contraindications like morbid obesity, osteoporosis and ossification of the posterior longitudinal ligament(OPLL).

Conclusion: This population-based study revealed that the prevalence of CDR has increased over time. A significant number of CDRs were performed with anterior or posterior cervical fusion, both in the elective and the emergent setting reflecting the use of the technique in an off-label manner.

222 Biomechanical Implications of Unilateral Facetectomy, Bilateral Facetectomy, and Partial Bilateral Facetectomy in Minimally Invasive Transforaminal Interbody Fusions

Laura A. Snyder, MD; Jennifer Lehrman, BSE, MS; Ramkumar Menon, MBBS, MS, DNB, MCh; Jakub Godzik, MD, MSc; Anna Newcomb; Brian P. Kelly, PhD

Introduction: Minimally invasive transforaminal interbody fusions can be performed with a unilateral facetectomy, bilateral facetectomy, or even a "partial bilateral facetectomy," during which on one side a full facetectomy is performed for interbody access, while on the other side the facet capsule is drilled to loosen the facet and decorticate bone for arthrodesis. In this study, we evaluated the biomechanical outcomes of unilateral facetectomy (UF), partial bilateral facetectomy (PBF), and complete bilateral facetectomy $(\mathrm{CBF})$.

Methods: 7 cadaveric specimens (L3-S1) were prepped for UF with full facet removal, hemilaminectomy, discectomy, and placement of pedicle screws $(6.5 \mathrm{X} 45 \mathrm{MM})$ at L4-L5. Without rods, distraction was performed via an instrumented distractor tool $(102 \mathrm{~N})$ and pure moment flexion $(7.5 \mathrm{Nm})$ test. A fixed interbody spacer was sized and placed into the specimen. Compression was then performed via an instrumented compressor tool(170N). Rods were locked into place via an instrumented compressor tool to $170 \mathrm{~N}$. Standard nondestructive flexibility tests $(7.5 \mathrm{Nm})$ were performed. Final lordosis angle was measured after rod lockdown with compression. Change in foraminal height was measured before and after posterior compression via compressor tool and final rod lockdown. The same procedure was performed for $\mathrm{PBF}$ and then $\mathrm{CBF}$ in all 7 specimens. Data was analyzed using RM-ANOVA $(\mathrm{P}<0.05)$.

Results: CBF demonstrated significantly greater $\operatorname{ROM}(8.00 \pm 3.50)$ in pure moment distraction than $\operatorname{UF}\left(7.24 \pm 3.33^{\circ}\right)$ $(\mathrm{p}=0.026)$. With final rod lockdown, CBF demonstrated significantly greater change in mean foraminal height $(1.90 \pm 0.62 \mathrm{~mm})$ than $\mathrm{UF}(1.00 \pm 0.45 \mathrm{~mm})(\mathrm{p}=0.037)$. Final lordosis angle achieved was greatest with $\mathrm{CBP}(3.74 \pm 0.70)$, slightly less with $\operatorname{PBF}(3.11 \pm 1.22)$, and lowest with $\mathrm{UF}(2.68 \pm 1.28)$, with statistical significance between all three approaches $(\mathrm{p}<0.04)$. 
Conclusion: CBF allows greater ability to distract for interbody placement, greater lordosis angles with final screw lockdown, and increased foraminal height when compared to UF and PBF. CBF and PBF add time, effort, and slightly increased risk to the minimally invasive transforaminal interbody fusion, however, they may improve biomechanical outcomes.

223 Minimally Invasive Lumbar Pedicle Screw Fixation using Cortical Bone Trajectory. A Prospective Cohort Study on Postoperative Pain, Functional Outcome, and Fusion Rates

Yi-Ren Chen MD, MPH; Rashad Jabarkheel, BS; Sayantan Deb, BA; Thu Lan Pham; Mahesh Patel, MD; Harminder Singh, MD

Introduction: Over the past decade, a new technique called cortical screw fixation has been developed, which allows for medialized screw placement through stronger cortical bone. Numerous studies have proven biomechanical equivalence or superiority for cortical screw versus pedicle screw fixation, but few have examined clinical outcomes.

Methods: We prospectively looked at the senior author's patients who underwent cortical versus pedicle screw fixation between 2013 and 2015 for lumbar degenerative disease. Eighteen patients underwent cortical screw fixation, and 15 patients underwent traditional pedicle screw fixation. We looked at postoperative pain, Oswestry Disability Index (ODI) and Roland Morris (RM) scoring pre and post-operatively, and fusion rates. An independent neuroradiologist assessed fusion by dynamic X-Rays.

Results: The cortical screw cohort showed a trend towards having less peak postoperative pain $(7.9$ versus $9, p=0.09)$. The average postoperative pain was similar between the two cohorts (4.7 vs $4.6, p=0.93)$. There was also no difference in pain six to 12 weeks after surgery ( 4.9 vs $4.9, p=0.8$ ). However, at six to eight months, the cortical screw cohort had worse pain compared to the pedicle screw cohort $(6.1$ vs $3.8, p=0.02)$. Cortical screw fixation resulted in a mean decrease of 27 from baseline ODI at 6-8 months $(\mathrm{P}=0.014)$, with a $70 \%$ fusion rate at 14.7 months.

Conclusion: The cortical screw patients showed a trend towards less peak pain in the short-term (one to three days postsurgery) and more pain in the long-term (six to eight months post-surgery) compared to pedicle screw patients. Both cohorts had a statistically significant reduction in pain levels compared to preoperative pain. Cortical screw fixation resulted in functional outcomes comparable to those seen with traditional pedicle screw fixation for lumbar degenerative disease, with a decrease of 27 from baseline ODI at 6-8 months.

224 Analysis of Complications with Staged Surgery for Less Invasive Treatment of Adult Spinal Deformity

Gregory M. Mundis, MD; Paul Park, MD; Khoi Duc Than, MD; Kai-Ming G. Fu, MD, PhD; Stacie Tran, BS, MPH; Juan S. Uribe, MD, FAANS; Adam S. Kanter, MD; David O. Okonkwo, MD, PhD; Pierce D. Nunley, MD; Robert Eastlack, MD; Richard G. Fessler, MD, PhD; Neel Anand, MD

Introduction: Spinal deformity surgery is often exceedingly invasive and lengthy. Therefore, staging surgery over separate operative days has been advocated to reduce complications. Staging is often used in minimally invasive treatment of adult spinal deformity (ASD). The impact of staging on complication rate between hybrid (HYB) and circumferential MIS (cMIS) procedures has not been well studied.

Methods: Patients who underwent staging, $=3$ levels treated, $=2 \mathrm{yr}$ follow-up were analyzed. 99 patients underwent staging including $53 \mathrm{cMIS}$ and $46 \mathrm{HYB}$. Propensity matching for levels fused resulted in 19 patients in each group. Intra- and peri-op (= 30days) complications were assessed.

Results: There were no significant difference in age, BMI, levels treated, OR time, LOS, VAS, ODI, CC, PT, PI-LL, SVA (Table 1). The overall complication rate was significant higher in HYB (89.5\% vs $52.6 \%, \mathrm{p}=0.012)$. Staged HYB patients were 7.6 times more likely to have a complication than staged cMIS patients. Three (15.8\%) HYB (implant, fracture, durotomy) but no cMIS intra-op complications occurred $(\mathrm{p}=0.071)$. There were more HYB $(52.6 \%)$ patients with peri-op complications than cMIS (21.2\%; $\mathrm{p}=0.044)$. Peri-op neurologic complications were more frequent in HYB $(36.8 \%)$ vs cMIS $(5.3 \%)(\mathrm{p}=0.017)$. Other complications did not differ significantly. 30 day re-operations were higher with cMIS $(10.5 \%)$ vs HYB $(0 \%)(\mathrm{p}=0.046)$. The 2 cMIS patients requiring a return to the OR had revisions due to a superficial infection and 1 sacral wound dehiscence.

Conclusion: In this propensity matched ASD cohort, MIS staged surgeries have fewer complications than HYB staged surgeries, but higher 30 day re-operations. Peri-op complications were significantly higher for HYB staged surgeries with neurologic adverse events being most common.

225 Fusion and Quality of Life Outcomes in Two consecutive Levels Non-segmental Minimally Invasive Transforaminal Lumbar Interbody Fusion (MiTLIF): A Feasibility Study

Sina Rajamand, DO; Matthew Bahoura; Jake Jasinski, D.O.; Ratnesh Mehra, D.O.; Doris Tong, MD; Teck Mun Soo

Introduction: Fusion rates for non-segmental MiTLIF using four instead of six screws has not been studied. We conducted a pilot study to determine the baseline fusion rate in patients who underwent two-level non-segmental MiTLIF.

Methods: Three consecutive lumbar vertebrae were instrumented using four instead of six pedicle screws without instrumenting the intervening vertebra in a non-segmental technique. We reviewed consecutive patients who underwent two-level minimally invasive lumbar fusion during 2015 in two hospitals under multiple surgeons. We excluded patients $<18$-year-old, with traumatic, oncologic, infectious etiology, had 5 pedicle screws inserted or previous spinal surgery at the same levels. The primary outcomes, fusion, SF-12, ODI, Back VAS were collected prospectively at $1 \mathrm{y}$ postoperatively by in-person interviews or by phone. Fusion was evaluated using the Suk fusion criteria. Secondary outcomes were readmission/reoperation within one month due to screw-related complications, estimated blood loss, OR time, and fluoroscopy time. This is a pilot study to evaluate baseline fusion rate for non-segmental vs. segmental two-level MiTLIF in this institution. Univariate analysis was used and $\mathrm{P}<0.05$ was considered significant. Sensitivity analysis was performed.

Results: We studied 116 patients. Seven patients were lost to follow up. $95(81.8 \%)$ patients had four-screws inserted. Fusion rate between the non-segmental vs. segmental groups was $87 \%$ vs. $86 \%$. Patient/perioperative characteristics, fusion and QoL outcomes were not significantly different between the two groups (table 1-3). Range of fusion was $82.1 \%-88.1 \%$ for non-segmental vs, $81.8 \%-86.3 \%$ for segmental instrumentation in sensitivity analysis.

Conclusion: We did not demonstrate any difference between segmental vs. non-segmental instrumentation for two-level MiTLIF regarding fusion or QoL. This is an expected outcome as this is a pilot study with both power and data distribution issues. We calculated our sample size to be 467 for our second-phase longitudinal cohort logistic regression study. 
226 The Effects of Spine Class on Length of Stay After Minimally Invasive Lumbar Fusion: Prospective Longitudinal Cohort Study

Dejan Slavnic, DO; Matthew Bahoura; Doris Tong, MD; Sina Rajamand, DO; Teck Mun Soo

Introduction: Length of stay (LOS) is a main factor in cost of inpatient care. We sought to demonstrate that preoperative spine class attendance decreases LOS after spinal fusion.

Methods: From 10/2016-10/2017, we prospectively followed consecutive patients who underwent elective minimally invasive thoracolumbar fusion in two hospitals under multiple surgeons. We excluded Medicare patients who stayed to satisfy insurance requirement for inpatient rehab and patients who developed surgical complications. Neurosurgical mid-level providers gave oral presentation with models and printed materials. The primary outcome was LOS. Secondary outcomes were patient satisfaction and expectation. The sample size was determined to detect $15 \%$ of LOS reduction. We used multiple linear regression with LOS as the dependent variable. The independent variables were spine class attendance, age, sex, number of comorbidities, surgeons' years of practice, surgical specialty, number of operative levels, and previous spinal fusion. The unstandardized coefficient (B) and its $95 \%$ confidence intervals were reported. The unstandardized coefficient showed change in the dependent variable for one-unit change in an independent variable when all other independent variables are kept constant. Bonferroni's correction was used and $p=0.0063$ was considered significant.

Results: Four hundred fifty one patients were studied. Fortyfive $(10.0 \%)$ attended the spine class. Patient demographics, perioperative characteristics and outcomes are presented in table 1,2, and 3. Multiple linear regression showed that age and surgical specialty (NS vs. Ortho spine) were significant predictors of LOS. B showed that increase in age was clinically insignificant $(B=0.0018$ day, 0.001) in increasing LOS. Switching from Orthopedic spine surgeons to Neurosurgeons caused a significant decrease in LOS $(\mathrm{B}=0.53, \mathrm{p}=0.001)$ (table 4).

Conclusion: Attending preoperative spine class does not decrease the LOS in patients undergoing lumbar spine fusion surgery. The only predictive factor of LOS is the surgical specialty of the surgeons.

227 Role of Minimally Invasive Lateral Interbody Fusion Surgical Technique in Thoracolumbar Fusion Revision Surgery

Karina Bach, BA; Konrad Bach, MD; Jacob Januszewski, DO; Juan S. Uribe, MD, FAANS

Introduction: Failed back syndrome, pseudarthrosis, loss of deformity correction, hardware failure, Adjacent Segment Failure (ASF), recurrent stenosis, and symptomatic recurrence are potential complications that can occur after index thoracolumbar fusion surgery and potentially require revision surgery. Minimally invasive (MIS) surgical techniques such as far lateral lumbar interbody fusion (MIS LIF) have seen widespread increase in treatment of various spinal pathologies. At present little data exists in the literature regarding the use of the MIS LIF in revision surgery.

Methods: We performed a retrospective review of a single center prospectively collected database of all patients with index thoracolumbar fusion requiring revision surgery between 2011 and 2016. Only patients treated with a procedure that included MIS LIF were studied. Indication for revision was recorded. Change in lumbar lordosis and fusion rates were the primary radiographic outcome measured. Clinical outcomes were measured using Oswestry Disability Index (ODI) and Visual Analog Scale (VAS). Complication rates were recorded. Statistical analysis was performed comparing preoperative and postoperative outcomes measures using paired students t-test. Minimum follow up was 3 months in order to capture early complications.

Results: Of 46 patients with prior thoracolumbar fusion requiring revision surgery 32 (16 female) met our inclusion criteria. The most common indication for revision surgery was adjacent segment failure $(64 \%)$. VAS improved by $2.48(\mathrm{p}<0.0005)$. ODI improved by $17.75 \%(\mathrm{p}<0.0005)$. Lumbar lordosis improved by $8.16^{\circ}(\mathrm{p}<0.0005)$. Overall complication rate was $21.8 \%$. All were transient in nature and related to the MIS far lateral approach.

Conclusion: MIS LIF represents a safe and effective alternative for thoracolumbar fusion revision procedures, with improvement in both clinical and radiographic outcomes.

228 Assessment of Clinical Outcomes and Efficacy of Minimally Invasive Transforaminal Lumbar Interbody Fusion (TLIF) in Degenerative Lumbar Scoliosis with Debilitating Back Pain

Mick J. Perez-Cruet, MD, MS; Esam A Elkhatib, MD, PhD; Elizabeth Abel, BS

Introduction: Assessment of clinical outcomes and efficacy of minimally invasive transforaminal lumbar inter-body fusion as a surgical management of selective pathological lumbar scoliotic segments has been studied in a few literature in deblitating back pain.

Methods: Forty one patients with degenerative scoliosis, (30(73.1\%) female, 11(26.9\%) male, average age 69 years old. Nine cases had BMI greater than $30 \mathrm{~kg} / \mathrm{m} 2$. Thirty one cases had lumbar scoliosis and 10 had thoraco-lumbar type. Twenty seven cases had spondylolisthesis GI of which 21 were L4-5, three were L3-4 and Three were L5-S1. All cases operated by TLIF were retrospectively reviewed between November 2011 to April 2017. categorized into 9 cases levodextro scoliosis, 9 cases degenerative, 22 cases dextro scoliosis, and 1 case juvenile scolioses. Oswestry disability index, Pain analogue scale, operative time, estimated blood loss (EBL), Cobb angle, operative complications and hospital stay (LOS) and fusion rates were analyzed.

Results: Follow-up mean time was $16.7 \pm 9.4$ months. All patients underwent single level TLIF. Mean operative time was $202.9 \pm 26.7$ minutes, mean EBL was $97.4 \mathrm{ml} \pm 55.8 \mathrm{ml}$ and mean LOS was 3.6 days \pm 3.1 days. Cobb angle preoperatively mean was $24.6 \pm 15.9$ which was not changed. VAS for pain significantly decreased from $8.83 \pm 1.6$ preop to $1.5 \pm 1$ at 3 months postoperative and $1.8 \pm 1.7$ at one year $(\mathrm{P}<0.05)$. The ODI mean score was $58.25 \pm 12.28$ preop decreased to $11.6 \pm 11.3$ at 3 months post-op and $14.5 \pm 13.7$ at one year. Complications rate was $7.3 \%(n=3)$, fusion rate was $>95 \%$ at 3 and 12 months follow-up based on dynamic plain radiographs. Re-operation rates was $4.8 \%(n=2)$.

Conclusion: Selective segment MI-TLIF surgery in scoliosis cases with debilitating low back pain is safe and effective procedure with excellent clinical outcomes and low complication rate.

229 Posterior Open-wedge Anterior Longitudinal Ligament Release: Cadaveric Technique and Early Clinical Experience

Michael Bohl, MD; Randy Hlubek, MD; David S. Xu, MD; Claudio Cavallo, MD; Mark C. Preul, MD; Steve W. Chang, MD; Jay D. Turner, MD, PhD; Juan S. Uribe, MD, FAANS; Udaya K. Kakarla, MD

Introduction: Anterior column realignment (ACR) is a powerful surgical technique for achieving spinopelvic balance in adult 
patients with sagittal plane deformities. We present an alternative strategy for focal deformity correction from a posterior-only approach. The technique comprises an opening wedge at the level of the disc space after posterior-based discectomy and anterior longitudinal ligament (ALL) release. The purpose of this study was to evaluate the feasibility, safety, and efficacy of a novel surgical technique, called the posterior open wedge ALL release (POWAR).

Methods: A cadaveric torso underwent POWARs at the L1-L4 disc spaces. Baseline measurements of the end-plate angle (EPA), anterior disc height $(\mathrm{ADH})$, and posterior disc height (PDH) were obtained. These measurements were then repeated after 3 stages of correction: posterior column compression alone, posterior column compression following Schwab grade 2 osteotomies, and posterior column compression following POWAR. Four patients underwent the described POWAR procedure for decompensating sagittal kyphotic deformities. Postoperative and 1-year outcomes are presented.

Results: In the cadaveric analysis, a sequential increase in $\mathrm{EPA}$ and $\mathrm{ADH}$, and a decrease in PDH, is demonstrated with each stage of correction. In-situ and ex-vivo investigation of the aorta and vena cava following anterior exposure revealed no injury to the great vessels from the disc distraction and ALL release. In the clinical series, each patient demonstrated large lordotic corrections associated with ADH expansion and a stable restoration of spinal alignment, with no vessel injury or plexopathy resulting from the POWAR procedure.

Conclusion: The POWAR is a new surgical technique that appears to be both safe and effective in the correction of adult sagittal deformities. This procedure provides an alternative to the 3 -column osteotomy for surgeons performing adult spinal reconstructions through an open, posterior-only approach.

230 The Minimally Invasive Transpsoas Lateral Lumbar Interbody Fusion: Determining Optimal Patient Positioning to Reduce Risk of Femoral Nerve Injury

Mena G Kerolus, MD; Anthony Frisenda; Anthony Serici; Ricardo B. V. Fontes, MD, PhD; Richard G. Fessler, MD, PhD

Introduction: The transpsoas lateral lumbar interbody fusion(LLIF)is a minimally invasive surgery technique used to achieve arthrodesis in the treatment of multiple lumbar spine pathologies.The risk of neurologic injury to the lumbar plexus and femoral nerve are high given its close relationship to the psoas muscle, especially at the L4/5 intervertebral disc(ID).The specific aim of this study was to identify the relationship of the femoral nerve to the L4/5 intervertebral disc space and optimal leg positioning that would provide the largest working zone for tubular dilation.

Methods: Five fresh, whole-body cadavers were dissected and the femoral nerve identified.A $6 \mathrm{~cm} 0.014$ in. guidewire was tied along the femoral nerve.A computed tomography (CT) scan was performed in the lateral position with the hip positioned at 0,45 , and 90 degrees with and without a 25 -degree prop in the contralateral costopelvic angle.Using Adobe Illustrator 2016, an imaging fusion technique was performed for analysis of the femoral nerve at different leg positions.

Results: A total of 10 femoral nerves were analyzed in 3 different leg positions with and without a prop for a total of 20 surgical positions(60 CT images).Each ID was divided into $8,0.5 \mathrm{~cm}$ zones with Zone 1 closest to the spinal canal.The femoral nerve was most frequently found in Zone $1(0-0.5 \mathrm{~cm})$ in 20 different leg positions. When the leg was positioned at 90 degrees, $11 / 20$ surgical positions offered the least amount of ID space.In $5 / 20$, the 45 and 90 degree leg positions were in the same position.In all cases, the neutral leg provided the largest exposure of the ID.There were no significant findings when using the prop on femoral nerve position.

Conclusion: Placing the lower extremity in a neutral position may provide the largest free disc space at $\mathrm{L} 4 / 5$ for tubular dilation. Further in vivo studies will need to be performed to confirm these findings.

231 The Effect of Preoperative Opioid Use on Patients Undergoing Stand-alone Lateral Interbody Fusion for Lumbar Degenerative Disc Disease

Alp Ozpinar, MD; Nima Alan, MD; David O. Okonkwo, MD, PhD; Adam S. Kanter, MD; D. Kojo Hamilton, MD

Introduction: There is a growing concern that the use of prescription opioids may predispose patients to hazardous opioid dependence. We hypothesized that the use of preoperative narcotics may lead to poorer patient outcomes following stand-alone lateral interbody fusion (LIF) for lumbar degenerative disc disease.

Methods: A consecutive series of patients from a single institution undergoing standalone LIF were retrospectively analyzed. Patients were categorized according to the presence or absence of prescribed preoperative opioid use $>6$ months. Outcomes included the Oswestry Disability Index (ODI), Visual Analogue Scale (VAS) and Short Form 36 Physical and Mental Summary Scores (SF36-PCS, SF36-MCS). Change values were calculated between postoperative and preoperative outcomes indices. Between-group comparisons were performed using analysis of variance.

Results: Overall in 48 patients, ( 23 male, 25 female) mean age was $48.2 \pm 14$.6-years; mean follow up 14.1 months mean number of levels 2 (1-4) and 22 (45\%) were prescribed preoperative opioids, with a mean oral morphine equivalent of $14.3 \pm 11.4 \mathrm{mg} /$ day. Mean preoperative ODI was $46.1 \pm 12.9$ (non-opioid 43.8 \pm 11.8 , opioid $48.9 \pm 14.0, \mathrm{p}=0.198)$. Mean postoperative ODI was $28.7 \pm 16.4$ (non-opioid $22.2 \pm 14.7$, opioid $36.5 \pm 15.6, \mathrm{p}=0.003$ ). Mean preoperative VAS $6.6 \pm 2.0$ (non-opioid $6.5 \pm 1.9$, opioid $6.7 \pm 1.0$, $\mathrm{p}=0.802$ ), and mean postoperative VAS was $4.1 \pm 2.3$ (non-opioid $3.2 \pm 2.1$, opioid $4.1 \pm 2.1, p=0.005$ ). There was a statistically significant change in postoperative from preoperative VAS, with the non-opioid group showing a greater subjective decrease in pain $(-3.3 \pm 2.4,-1.6 \pm 2.6, p=0.032)$. There was a trend in the change in postoperative from preoperative ODI, with the non-opioid group showing a greater magnitude of improvement $(-21.6 \pm 17.6$ vs. $-12.4 \pm 17.4, \mathrm{p}=0.089)$. Changes scores for SF36-PCS and SF36MCS were not statistically significant by opioid use group.

Conclusion: Preliminary data from a single center consecutive study onpatients undergoing LIF for lumbar degenerative disc disease suggest that patients prescribed preoperative opioids may suffer increased postoperative lumbar pain disability and subjective pain compared to patients not on preoperative opioids. Future studies are needed for confirmation.

232 A New Minimally Invasive Device to Deliver Stem Cells to the Spinal Cord of Patients with ALS.

J. Patrick Johnson, MD, MS; Pablo Avalos; Doniel Drazin, MD, MA; Harish Babu, MD, PhD; Chirag Gadkary Patil, MD, MS; Margaret Allred; Robert Lewis; Robert Baloh; Clive Svendsen

Introduction: Amyotrophic lateral sclerosis (ALS) results in loss of motor neurons in the spinal cord and there is no known etiology, treatment, or cure. Preclinical studies have shown that glial cell line-derived neurotrophic growth factor (or GDNF) can protect motor neurons from damage in animal models that may include ALS. There is an ongoing Phase 1 clinical trial https:// 
clinicaltrials.gov/show/NCT02943850 designed to deliver GDNF producing stem cells into the spinal cord of patients with ALS that could potentially slow the death of motor neurons causing paralysis.

Methods: Patients with ALS are treated with an investigational new device that utilizes a minimally invasive surgical retractor that is anchored to the operating room table. The device has precision stereotactic adjustment capabilities designed for delivery of stem cells into the lumbar region of the spinal cord in these FDA approved experimental procedures. Radiologic localization of the 4 th and 5th lumbar segments of the spinal cord are targeted from MRI measurements in the ventral horn region of the spinal cord.

Results: Six patients with ALS have been treated so far in this Phase 1 non-randomized study, and no significant adverse events related to the device have been seen. There has been short-term transient pain in the lower extremity. Post-operative imaging has not demonstrated any changes in the spinal cord parenchyma

Conclusion: Precision delivery of these stem cells into the spinal cord with a new minimally invasive device appears to be feasible based upon early results of this study. Completion of this Phase 1 study is ongoing and will be completed in the next calendar year and then final results will be reported. At this early stage, technical and safety goals associated with this FDA approved minimally invasive investigational device appear to be attainable.

233 The Role of Minimally Invasive Techniques in Scoliosis Correction Surgery

Jack Goergen, BS; Michael Cloney, MD; Angela Marie Bohnen, MD; Zachary Adam Smith, MD; Tyler R. Koski, MD; Nader S. Dahdaleh, MD

Introduction: Minimally invasive techniques have been developed and included among the treatment modalities used for scoliosis. However, the literature comparing minimally invasive surgery (MIS) to open surgery for scoliosis correction is limited.

Methods: We retrospectively examined records from 207 consecutive scoliosis correction surgeries at our institution between 2009 and 2015. We collected data on patient demographics, procedure characteristics, hospital course, and 30-day rates of readmission, reoperation. MIS scoliosis corrections were compared to open scoliosis corrections

Results: MIS patients had a lower median number of levels fused (4.0 v. 9.0, ? 5.0 [2.0, 7.0], p<0.0001, Figure 1). There was significantly more variance in the number of levels fused among open surgery patients $(\mathrm{p}<0.0001)$. MIS patients had shorter surgeries (287.0 minutes v. 433.0 minutes, HR 2.319 [1.604, 8.342], $\mathrm{p}=0.0023$, Figure 2 ), shorter lengths of stay (4.5 days v. 8.0 days, HR 3.032 [3.725, 22.61], $\mathrm{p}<0.0001$, Figure 3), were less likely to be admitted to the ICU (OR 0.08779576 [0.02348702, 0.3281854], $\mathrm{p}<0.0001$ ), and had shorter ICU stays (19.0 hours v. 48.5 hours, HR $5.174[5.200,866.7], p=0.0015)$. On multivariable regression, the number of levels fused predicted selection for MIS procedure (OR 0.6079009 [0.4340611, 0.8513629], $\mathrm{p}=0.004)$, and there was a trend toward significance for age (OR 1.076323 [0.9977851, 1.161042], $\mathrm{p}=0.057$ ), and having private insurance (OR 3.735077 [0.9058839, $15.40021], \mathrm{p}=0.068$ ), undergoing a posterior approach (OR 3.43426 $[0.8365153,14.09913], \mathrm{p}=0.087)$, and not requiring surgical decompression (OR 0.1319887 [0.0147237, 1.183196], p=0.070). Age predicted ICU admission and VTE. Undergoing MIS surgery was not an independent predictor of any of the outcomes analyzed.

Conclusion: Patients undergoing MIS scoliosis correction had shorter surgeries, shorter lengths of stay, and shorter and fewer ICU stays, but there was a significant selection effect. Accounting for other clinical variables, undergoing MIS surgery did not independently predict any of the outcomes analyzed.
234 Which Cervical Artificial Disc Should We Choose?: An In Vivo Study.

Hoon Choi, MD, MS; Jamie Baisden, MD; Narayan Yoganandan, $\mathrm{PhD}$

Introduction: There has been increasing attention on cervical arthroplasty as an alternative to arthrodesis as a way to preserve motion, reduce adjacent level degeneration, avoid reoperation, and improve patient-reported outcome. There are three types of cervical artificial discs on the market: constrained, semi-constrained, and unconstrained. There is no federally funded in vivo study that directly compares different cervical artificial discs.

Methods: We performed cervical arthroplasty at C3/4 level in 14 healthy female adult Alpine goats. Five goats received Bryan (Medtronic) (constrained disc); five goats received ProDisc-C (DePuy Synthes Johnson\&Johnson) (semi-constrained); and four goats received Mobi-C (LDR Biomet Zimmer) (unconstrained). These goats were monitored in a unit for six months with regular cervical spine radiographs.

Results: All 14 goats tolerated the surgery well. All had satisfactory placement of hardware. No migration of implant occurred in the Bryan and ProDisc-C groups. $100 \%$ migration rate (4/4) was observed with Mobi-C within the first week, requiring implant removal and arthrodesis with an interbody and anterior plating. Heterotopic ossification was observed at six months in $40 \%$ of goats with Bryan (2/5). Partial heterotopic ossification (with motion preservation) was seen in $20 \%$ of goats with ProDisc-C $(1 / 5)$. Cervical motion was ultimately preserved at six months in no goats with Mobi-C (due to fusion), $60 \%$ of goats with Bryan, and $100 \%$ of goats with ProDisc-C.

Conclusion: Unconstrained cervical artificial discs are prone to anterior migration. Constrained discs are more likely to develop heterotopic ossification than semi-constrained discs. Semiconstrained discs are most likely to preserve motion.

235 Quantity and Quality of Motion of a Mobile-core Cervical Disc Prosthesis in Two-level Arthroplasty

Robert M. Havey; Saeed Khayatzadeh, PhD; Leonard I. Voronov, MD, PhD; Gerard Carandang, MS; Frank M. Phillips, MD; Avinash G Patwardhan, PhD

Introduction: The FDA recently approved a mobile-core cervical total disc arthroplasty (TDA) for 1- and 2-level implantations. The design includes a UHMWPE mobile core that articulates with the lower metal $(\mathrm{Co}-\mathrm{Cr})$ endplate in a planar bearing, allowing anterior-posterior and lateral translation. The core articulates with the superior metal endplate in a spherical bearing. This disc may be classified as a 5 degrees-of-freedom disc that is unrestrained since it does not resist angular or translational motions until reaching the built-in stops. The purpose of this study was to investigate the motion response of this mobile-core TDA when implanted in human cadaveric spines in 1- and 2-level reconstructions.

Methods: We tested eight cadaveric specimens (C3-T1) (age:42 \pm 6 ) under physiologic loads in flexion-extension, lateral bending, and axial rotation; first intact, then after implantation of TDA at C5-C6 (1-level), and C6-C7 (2-level).

Results: Flexion-extension range of motion (ROM) increased after TDA from $13.7 \pm 4.3$ to $16.7 \pm 4.2$ degrees $(\mathrm{P}<0.01)$. In 6 of 16 implanted segments postoperative flexion-extension ROM was greater than 20 degrees (Figure 1A). In 8 of 16 segments, the kinematic signature reflected acceptable quality of motion (Figure 1B), while the remaining 8/16 segments demonstrated instability, or large increases in motion for a small applied moment (Figure 1C). 
Contrary to intact specimen behavior, post-TDA motions at C5-C6 and $\mathrm{C} 6-\mathrm{C} 7$ were sequential (not synchronized) in 5/8 specimens; motion occurred first at C5-C6 followed by C6-C7.

Conclusion: In 6/16 implanted segments the postoperative flexion-extension motion was significantly larger than the adjacent C4-C5 segment, far exceeding physiologic normative ROM in vivo. These segments had above normal preoperative ROM and the unrestrained nature of this prosthesis likely allowed excessive motion to occur post-implantation. The mobile-core design does not lend itself to controlled motion, leading to poor quality of motion in some segments, a behavior noted previously for the lumbar mobile-core prosthesis.

236 Cost-effectiveness Analysis of Minimally Invasive (MI) Versus Open Transforaminal Lumbar Interbody Fusion (TLIF) for Grade I Degenerative Spondylolisthesis

Juneyoung L Yi, MD, MSPH; Brandon K Bellows, PharmD, MS; Erica Fay Bisson, MD, MPH, FAANS; Jian Guan, MD; Praveen V. Mummaneni, MD; Panagiotis Kerezoudis; Steven D. Glassman; Kevin T. Foley, MD, FACS, FAANS; Jonathan Slotkin, MD; Eric A. Potts, MD; Mark Edwin Sha

Introduction: Minimally invasive lumbar fusion surgery has become popular due to lower blood loss, shorter hospital stay, and shorter time to ambulation. Despite short-term benefits, there is no difference in long-term patient-reported outcomes, fusion rates, or reoperation rates. It is unknown if the improvement in shortterm outcomes outweighs the increased surgical costs and lack of difference in long-term outcomes. Furthermore, prior economic analyses have shown MI TLIF is cost-effective, but these did not include a decision analytic model with interim utilities or results beyond two years. This study evaluates the cost-effectiveness of first-time MI versus open TLIF for grade I degenerative lumbar spondylolisthesis from a US payer perspective over a lifetime.

Methods: A Markov model was used to estimate the costs (2017 US\$) and quality-adjusted life-years (QALYs) for open and MI TLIF. Probabilities and utilities were derived from published US literature. Costs (2017 US\$) were derived from published US prospective studies, the Centers for Medicare \& Medicaid Services Physician Fee Schedule, and the Healthcare Cost and Utilization Project. A willingness-to-pay threshold of $\$ 100,000$ per QALY was used to determine cost-effectiveness. The model assumed patients could receive only one revision surgery during their lifetime, when needed, for pseudoarthrosis, adjacent segment disease, or recurrent stenosis. Postoperative complications could occur after each surgery. These included RBC transfusion, CSF leak, hardware complication, hematoma, wound complication, and new neurologic deficit.

Results: The base case analysis showed MI TLIF was more costly than open TLIF over a lifetime $(\$ 106,808$ vs. $\$ 78,252)$. However, it was also more effective than open TLIF over a lifetime (10.04 vs. 9.48 QALYs). The incremental cost effectiveness ratio (ICER) of MI TLIF was $\$ 51,085$ per QALY gained.

Conclusion: For the surgical treatment of grade I degenerative spondylolisthesis over a lifetime from a US payer perspective, MI TLIF is cost-effective when compared to open TLIF.

237 Incidence of Femoral Nerve Palsy Following Standalone L4-5 Minimally Invasive Lateral Lumbar Interbody Fusion

Nima Alan, MD; Nitin Agarwal, MD; David J Salvetti, MD; Balzer Jeffrey, PhD; D. Kojo Hamilton, MD; David O. Okonkwo, $\mathrm{MD}, \mathrm{PhD}$; Adam S. Kanter, MD
Introduction: It has been opined that LLIF at L4-5 carries the greatest risk of eliciting femoral nerve injury. We aimed to evaluate the incidence of femoral nerve palsy following stand-alone L4-5 LLIF.

Methods: A retrospective review of a prospectively maintained database was conducted of patients who underwent standalone LLIF at L4-5 at our institution between 2009 and 2015. All cases were monitored intraoperatively using free-running and triggered-EMG. Patients with posterior segmental fusion, staged deformity correction surgery, or multi-level stand-alone lumbar LLIF were excluded. Psoas weakness (hip flexion; secondary to pain and/or psoas stretch/injury) and femoral nerve function (hip flexion/knee extension), were evaluated pre- and post-operatively and throughout follow- up until reported normal.

Results: Sixty-nine patients (27 male, 42 female) with an average age of 53 years were included in the study. Transient hip flexion weakness was observed in 15 patients $(21.7 \%)$. All patients had full recovery to $5 / 5$ strength; 11 recovered at 6 weeks, 3 at 12 weeks and one patient at 24 weeks. Three patients $(4.3 \%)$ had simultaneous hip flexion (HF) and knee extension (KE) weakness. Of these three patients, two patients recovered from $3 / 5$ strength in both muscle groups to $5 / 5$ at 3 months. One patient developed $4 / 5$ weakness in both $\mathrm{HF}$ and $\mathrm{KE}$, which improved to $5 / 5$ at four months. No significant EMG discharges or low thresholds were noted. The location of psoas muscle was within the previously well-described operative zone without anterior transposition.

Conclusion: Femoral nerve palsy was transient with full recovery within 4 months maximum and not associated with significant EMG events. These results suggest axonotmetic peripheral nerve injury to the femoral nerve. Despite the reported propensity for a higher incidence of femoral nerve injury at L4-5, stand-alone minimally invasive LLIF can be safely and effectively performed at this level in a properly selected patient population.

238 Minimally Invasive Versus Open Fusion for Grade I Degenerative Lumbar Spondylolisthesis: Analysis of the Quality Outcomes Database

Praveen V. Mummaneni, MD; Erica Fay Bisson, MD, MPH, FAANS; Panagiotis Kerezoudis; Andrew Kai-Hong Chan, MD; Steven D. Glassman; Kevin T. Foley, MD, FACS, FAANS; Jonathan Slotkin, MD; Eric A. Potts, MD; Mark Edwin Shaffrey, MD, FAANS, FACS; Christopher I. Shaffr

Introduction: Lumbar spondylolisthesis is a degenerative condition that can be surgically treated with either open or minimally invasive decompression and instrumented fusion.

Methods: We queried the national, multi-center, Quality Outcomes Database (QOD) registry from July 2014 through December 2015 for patients undergoing posterior lumbar fusion for grade I degenerative spondylolisthesis.

Results: A total of 345 patients (Open-254, MIS-91) from 11 participating sites were identified in the QOD. Follow-up rate at 12 months was $84 \%$ (Open-83.5\%, MIS-85\%). Overall, baseline patient demographics, comorbidities and clinical characteristics were similarly distributed between the two cohorts. Two hundred fifty seven patients underwent 1-level fusion (Open-181, MIS-76) and eighty eight patients underwent 2-level fusion (Open-73, MIS15). Patients in both groups reported significant improvement in all primary outcomes (all $\mathrm{p}<.001$ ). MIS was associated with significantly lower mean intraoperative blood loss and slightly longer operative times in both 1- and 2-level fusion subgroups. Although the length of stay was shorter for MIS 1-level cases, this was not significantly different. No difference was detected with regards to the 12-month patient reported outcomes between the one level mis versus the one level open surgical groups. However, change in functional outcome scores for patients undergoing 2-level fusion 
was notably larger in the MIS cohort for ODI (-27 vs. $-16, \mathrm{p}=0.1)$, $\operatorname{EQ5D}(0.27$ vs. $0.15, \mathrm{p}=0.08)$, and NRS-BP ( 3.5 vs $-2.7, \mathrm{p}=0.41)$; statistical significance was shown only though for NRS-LP scores $(-4.9$ vs. $-2.8, \mathrm{p}=0.02)$. On risk-adjusted analysis for one level fusion, open versus minimally invasive approach was not significant for 12-month PROs, length of stay and 90-day return to work.

Conclusion: Significant improvement was found in all functional outcomes in patients undergoing open or MIS fusion for lumbar spondylolisthesis. No difference was detected between the two techniques for 1-level fusion in terms of patient reported outcomes, length of stay and 90-day return to work. However, patients undergoing 2-level MIS fusion reported significantly better improvement in NRS-LP at 12 months.

239 The Impact of Revision Surgery on Patients Undergoing MIS Correction of Adult Spinal Deformity: A Multi-Center Study

Michael Gerling; Gregory M. Mundis, MD; Juan S. Uribe, MD, FAANS; Paul Park, MD; Praveen V. Mummaneni, MD; Kai-Ming G. Fu, MD, PhD; Stacie Tran, BS, MPH; Khoi Duc Than, MD; Pierce D. Nunley, MD; David O. Okonkwo, MD, PhD; Richard G. Fessler, MD, PhD; Neel Anand

Introduction: Though minimally invasive surgical (MIS) approaches for adult spinal deformity (ASD) correction have favorable reoperation rates compared to traditional open techniques, the impact of revision surgery on outcomes has not been defined. This study reviews MIS surgical outcomes in patients requiring revision surgery (RO) versus those with no reoperation (NRO) after more than 2 years follow-up.

Methods: Patients underwent MIS deformity correction were used to analyze patient characteristics, radiographic parameters and outcomes in RO compared to NRO. MCID of 12.8 was used to compare significance of changes in ODI. Minimum 2 year followup was required.

Results: 184 patients met criteria with a $25 \%$ reoperation rate (46 RO; $138 \mathrm{NRO}$ ). There was no significant difference in age for RO vs. NRO (61.3 vs. 59 ; $p>0.477)$, BMI (28.5 vs. $26.6 ; \mathrm{p}>0.109$ ) and levels instrumented (7.1 vs. 6.5; p>0.257). Follow-up was longer for RO (Ave 44.7 vs. 36.4 months). RO had worse baseline preoperative deformity, PI-LL (20.3 vs. 14.3; $\mathrm{p}=0.017)$ and SVA (53.7 vs. 38.9; $\mathrm{p}=0.124)$. Baseline outcome scores were similar for VAS Leg (5.4 vs. $5.7 ; \mathrm{p}=0.435)$, VAS back ( 6.8 vs. $7.0 ; \mathrm{p}=0.2)$, and ODI ( 50 vs. $51.5 ; \mathrm{p}=0.644)$. Postop Final follow-up scores were similar, VAS Leg ( 2.5 vs. $2.5 ; \mathrm{p}=0.983)$, VAS back ( 3.9 vs. $3.8) ; \mathrm{p}=0.405$ ), and ODI ( 37 vs. $31.6 ; \mathrm{p}=0.136$ ). There was less change in ODI ( 13.8 vs. $19.9 ; \mathrm{p}=0.014)$ in favor of NRO patients, without a difference in MCID (61.2\% vs.50.0\%; $\mathrm{p}=0.184)$.

Conclusion: Patients with and without revision surgery have similar outcomes after MIS correction of ASD. VAS pain scores for leg and back pain, and ODI scores are similar between groups at baseline and after two years follow-up. There is an increased ODI benefit found in patients without reoperation, but it does not reach clinical significance. This study suggests that revision surgery may have less similar impact on clinical outcomes in this patient populationas patients who did not need revision surgery.

240 Limitations of the Patient Experience Reports in Spine Surgery: Analysis of Seven Thousand Four Hundred and Eighty Five $(\mathbf{7 , 4 8 5})$ Surveys

Nitin Agarwal, MD; Andrew M Faramand, MD; Johanna Bellon, Ph.D.; Jeffrey Borrebach, M.S.; D. Kojo Hamilton, MD; David O. Okonkwo, MD, PhD; Adam S. Kanter, MD

Introduction: The Clinician and Group Consumer Assessment of Healthcare Providers and Systems (CG-CAHPS) is a standardized patient experience survey that is used to evaluate the quality of care delivered by physicians. We sought to determine which factors influenced CG-CAHPS scores for spine surgery.

Methods: A retrospective study of prospectively obtained data was performed to evaluate CG-CAHPS scores. Between May 2013 and May 2017, all patients 18 years of age or older with an office-based encounter with a neurosurgeon (7 spine surgeons and 18 from other subspecialties) received a CG-CAHPS survey. The overall physician rating is a scale of zero to ten. The likelihood to recommend and physician communication domains are scored based on a three point Likert scale ('Yes, definitely', 'Yes, somewhat', 'No'). Answers of 'Yes, definitely' are considered "Top Box" scores. Statistical tests were performed with Chi-square test using SPSS 24 (IBM, Armonk, NY).

Results: Seven thousand four hundred and eighty-five $(7,485)$ patient surveys (2,319 spine and 5,166 other subspecialties) were collected from patients presenting to the outpatient offices of an attending neurosurgeon. Analysis of the overall physician rating showed that $81.1 \%$ of spine surgeons received a top box score, where as $86.2 \%$ of the other neurosurgeons received a Top Box response $(\mathrm{p}=<0.001)$. A similar difference was observed with the domains of likelihood to recommend and physician communication. Overall physician rating was also significantly influenced by the general and mental health of the patients surveyed $(p=<0.001)$. For spine surgeons seeing patients at 2 or more different facilities, the scores with respect to location were significantly different in all domains for each provider ( $\mathrm{p}<0.001)$.

Conclusion: Factors aside from just the quality of care provided may affect physician ratings obtained via patient experience surveys. This information should be considered as payers, government, and health systems design performance programs based on patient experience scores.

\section{Musculoskeletal Disorders in Surgeons: A Literature} Review

Yuping Derek Li, MS; Gurvinder Kaur, MD; Benjamin Smith; Aruna Ganju, MD

Introduction: The medical community is becoming increasingly aware of surgeons' physical stress and fatigue in the operating room environment. Spine surgery requires surgeons to adopt non-ergonomic positions for variable amounts of time in attempt to decompress neural elements, stabilize the spinal column, and correct spinal deformity. While patient outcomes are a focus of healthcare in this country, the impact of surgery on the surgeon has received far less attention. This study aims to gain insight into the epidemiology of musculoskeletal complaints among surgeons as well as highlight various interventions that are currently being developed.

Methods: We conducted a literature search for articles published between 1990-2017 with a primary focus on epidemiology of work-related musculoskeletal complaints among surgeons. The key words utilized in the search were the following: (1) surgeon, (2) musculoskeletal pain, (3) occupational disease, (4) epidemiology, and (5) ergonomics. Subsequently, the reference results were examined for additional studies.

Results: In survey-based studies, 50-86\% of surgeons reported some physical symptoms or discomfort. The most common symptoms reported were neck pain/strain, low back pain, and radiculopathy. One study reported $43 \%$ of surgeons had to take breaks from operating due to their symptoms; another study reported $26.7 \%$ of surgeons took time off of work due to these work-related injuries. Additionally, there is no clear consensus on the cumulative impact of surgeons' years of experience or annual caseload with work- 
related musculoskeletal disorder. Current strategies being utilized include microbreaks during surgery as well as physical support devices.

Conclusion: The impact of surgery on the surgeon has only recently begun to be investigated. Given the significant effect on surgeons, particularly spine surgeons, better kinematic studies of the surgeon and ergonomics of the operating room are needed. In addition, preliminary interventions including microbreaks and body supports have shown promise.

242 Changes in the Efficiency of Whole-Brain Network in Spinal Cord Injury: A Resting-state fMRI Study Using Graph Theory

Mayank Kaushal, MD, MBA; Akinwunmi Oni-Orisan, MD; Gang (Jeff) Chen; Wenjun Li, PhD; John Leschke; B. Douglas Ward, MS; Benjamin Kalinosky, PhD; Matthew Budde, PhD; Brian Schmit, PhD; Shi-Jiang Li, PhD; Vaishnavi Muqeet, MD; Shekar N. Kurpad, MD, PhD

Introduction: The application of a graph theoretical framework divides the resting-state functional MRI (rs-fMRI) dataset into "nodes" and "edges" that allows for the calculation of quantifiable network metrics. These metrics can be compared between clinical populations of interest and controls to evaluate connectivity alterations in brain networks. The present analysis highlights changes to the efficiency of whole-brain network in patients with spinal cord injury (SCI).

Methods: Subsequent to the IRB approval, 15 subjects with chronic, complete cervical SCI and 15 neurologically intact controls were scanned. The raw imaging datasets were preprocessed and then parcellated into 264 regions of interest (ROIs) based on the functional atlas from Power et al. Correlation analysis was performed between the average time series of every pair of ROIs to construct connection matrices. Local efficiency (LE) and global efficiency (GE) were then calculated at incremental cost thresholds (\% of total possible connections) and compared between the study groups.

Results: The whole-brain resting-state network showed significant differences for GE and LE, measuring the ability to transmit information at global and local level, respectively, between the two groups at multiple cost thresholds (Figure 1). GE was increased while LE was reduced in SCI compared to controls.

Conclusion: The differences in GE and LE, evaluating network attributes of integration and segregation, respectively, highlights the applicability of graph theory in conducting largescale network analysis in clinically relevant neurosurgical patient populations. The findings illustrate the inherently plastic nature of the brain, which leads in persistent connectivity alterations, causing global and local changes to information transmission following distant injury to the spinal cord. This hints to the potential of quantitative metrics in the development of noninvasive imaging biomarkers for improving prognostication after SCI and measurement of functional recovery.

243 Aggressive Surgical Treatment and Critical Care Are Associated with Improved Neurologic Recovery in Complete SCI

Anthony Michael DiGiorgio, DO, MHA; John Frederick Burke, MD, PhD; Ethan A. Winkler, MD, PhD; John K. Yue, BA; Laura Benjamin Ngwenya, MD, PhD; Jason Talbott, MD, $\mathrm{PhD}$; Adam Ferguson, PhD; Michael Beattie, PhD; Jacqueline Bresnahan, PhD; Jenny Haefeli; William Whet

Introduction: Spine Injury Association (ASIA) A injury, or a complete spinal cord injury (SCI), is a devastating diagnosis. This can severely shorten the life expectancy of a patient and is costly to society. Patients who present as an ASIA A have very low rates of improvement. The literature reports improvement of one ASIA grade range from $11-40 \%$ of patients by time of discharge. The object of our study was to determine if aggressive surgical treatment and critical care are associated with improved neurologic recovery in complete SCI.

Methods: The Transforming Research and Clinical Knowledge in SCIs study is a retrospective observational study conducted by the University of California at San Francisco Brain and Spinal Injury Center. The database was queried for all spinal cord injuries from 2004 2015. Time to surgical intervention was collected along with presenting and discharge ASIA grades.

Results: 62 patients were enrolled in the study and 28 of these were ASIA A at presentation. $57.1 \%$ of complete SCI patients improved at least one grade by discharge and $50 \%$ improved at least two grades. 9 patients were operated on within 12 hours and 19 after 12 hours. $89 \%$ of the patients operated on within 12 hours improved at least one AIS grade compared to $42 \%$ of the patients operated on after 12 hours $(\mathrm{p}=.039)$.

Conclusion: Aggressive surgical and critical care management of complete spinal cord injury can lead to higher improvement rates than those previously reported in the literature. This has the potential for long term improvement in quality of life as well as substantial cost savings.

244 Histological Correlates of in Vivo Diffusion Tensor Imaging in Rat Spinal Cord Injury Cranial to the Lesion

Olesya Motovylyak, BS; Brian Schmit, PhD; Shekar N. Kurpad, MD, PhD; Matthew Budde, PhD

Introduction: The objective of this study was to understand the physiological basis of diffusion changes in the spinal cord cranial to injury in rats. Diffusion Tensor Imaging (DTI) has been widely used in the field of spinal cord injury, but its utility is limited by hardware artifacts and injury factors such as inflammation, hemorrhage, and edema at the site of injury. Previous studies have documented ex vivo diffusion changes away from injury, but the pathological causes of those changes require further exploration [1][2].

Methods: Female Sprague-Dawley rats $(n=40)$ underwent a controlled T8 contusion injury at one of four severities. The rats underwent in vivo DTI scans at 2, 30, and 90 days post injury using a 9.4T Bruker horizontal bore scanner and were assessed on the Basso, Beattie, and Bresnahan (BBB) scale to determine functional recovery. Diffusion weighted images were acquired with a 4-shot echo-planar spin-echo sequence $(\mathrm{TE}=27 \mathrm{~ms}$; $\mathrm{TR}>1800$ $\mathrm{ms})$ and custom routines were used to reconstruct and register DTI maps. Formalin fixed samples from C5 segment were stained with primary antibodies for non-phosphorylated neurofilaments (SMI32), marker of injured axons. Number of stained objects was used to quantify SMI32 staining on digital images. Two-way ANOVA and linear regression were used to determine significant differences between time and injury severities for imaging and histological measures.

Results: White matter region of interest analysis revealed that SMI32 staining has a significant effect of time after injury $(\mathrm{p}<0.0005)$, injury severity $(\mathrm{p}<0.0005)$ and interaction $(\mathrm{p}=0.006)$. The staining was most widespread throughout ventrolateral white matter at 2 days and diminished over time. SMI32 was correlated with $\mathrm{BBB}$, particularly at the early time point; axial diffusivity (AD), on the other hand, was correlated with BBB scores at 90 days (Figure 1).

Conclusion: AD was a better predictor of injury severity 
chronically, while axonal damage correlated better with locomotor recovery acutely.

245 Simultaneous Lateral Interbody Fusion and Pedicle Screws (SLIPS) with CT-guided Navigation: Technical Note

Jonathan N Sellin, MD; Rory R. Mayer, MD; Marcus Hoffman, MD; Alexander E. Ropper, MD

Introduction: We present the operative technique for "anterior to psoas" lumbar interbody fusion (ATP-LIF) with truly simultaneous posterior lumbar percutaneous pedicle screw fixation using intraoperative CT-guided navigation.

Methods: This technique capitalizes both on the more oblique incision and approach used in the ATP-LIF procedure, as well as the anatomic clarity gleaned from intraoperative CT-guided navigation, to allow for simultaneous placement of pedicle screws in the lateral position during the ATP-LIF procedure without the need for fluoroscopy.

Results: A total number of 4 cases were included. Mean patient age was 71.8 (range, 70-73). There were 0 male and 4 female patients. The primary diagnosis was spondylolisthesis in 3 patients and degenerative disc disease in 1 patient. All cases were single level fusions. All cases were performed at the L4-5 level. Mean operative time was 138 minutes (SD 16.7, range 129-163). Average estimated total blood loss was $73.8 \mathrm{cc}$ (SD 56.5, range 20-150). Average hospital stay was 6.5 days (range 3-12). Mean radiation exposure was $63.2 \mathrm{mGy}$ (range 45.36-110.22). At total of 14 pedicle screws were placed in one patient, only a unilateral pedicle-rod construct was pursued. Two lateral pedicle breaches $(2 / 14,14 \%)$ were identified; all other pedicle screws were within the pedicle

Conclusion: This approach allows the two procedures to proceed in truly simultaneous fashion and further reduce operative times and radiation exposure with comparable results and little perioperative morbidity seen in our nascent experience.

246 Optical Topographic Imaging for Spinal IntraOperative Three-Dimensional Navigation in Minimallyinvasive Approaches: Initial Pre-Clinical and Clinical Experience

Daipayan Guha, MD; Raphael Jakubovic, MSc; Albert Yee, MD; Michael G. Fehlings, MD, PhD, FRCS(C), FACS; Victor Xiao Dong Yang, MD, PhD, MSc, BSc

Introduction: Computer-assisted three-dimensional navigation may guide spinal instrumentation. A novel optical topographic imaging (OTI) system, developed in our laboratory, offers comparable accuracy and significantly faster registration relative to current navigation systems, in open posterior thoracolumbar exposures. Here, we explore the utility of OTI in minimallyinvasive (MIS) spinal approaches.

Methods: Mini-open midline posterior exposures were performed in four human cadavers. Square exposures of size 25, 30, 35 , and $40 \mathrm{~mm}$ were registered to a preoperative CT scan. Screw tracts were fashioned using a tracked awl and probe, and instrumentation placed. Navigation data were compared to screw positions on postoperative CT imaging, and absolute translational and angular deviations computed. In-vivo validation was performed in eight patients, with mini-open thoracolumbar exposures and percutaneous placement of navigated instrumentation.

Results: For 37 cadaveric screws, absolute translational errors were $(1.79 \pm 1.43 \mathrm{~mm})$ and $(1.81 \pm 1.51 \mathrm{~mm})$ in the axial and sagittal planes, respectively; absolute angular deviations were $(3.81 \pm 2.91$ ?) and $(3.45 \pm 2.82$ ?), respectively (mean \pm SD). Errors were similar across levels and screw types. The number of surface points registered by the navigation system, but not exposure size, correlated positively with the likelihood of successful registration $(\mathrm{OR}=1.02,95 \%$-CI 1.009-1.024, $\mathrm{p}<0.0001) .55$ in-vivo thoracolumbar pedicle screws were analyzed. Overall (mean \pm SD) axial and sagittal translational errors were $(1.79 \pm 1.41 \mathrm{~mm})$ and $(2.68 \pm 2.26 \mathrm{~mm})$, while axial and sagittal angular errors were $(3.63 \pm 2.92$ ?) and $(4.65 \pm 3.36$ ?), respectively. There were no critical radiographic breaches, nor any neurovascular complications from any placed screws.

Conclusion: OTI is a novel navigation technique previously validated for open posterior exposures, which has comparable accuracy for mini-open MIS exposures. The likelihood of successful registration is affected more by the geometry of the exposure than its size.

247 Smart Human Neural Stem Cells to Modify Scar and Optimize Regeneration in Chronic Traumatic Cervical Spinal Cord Injury

Christopher Saagar Ahuja, MD; Mohamad Khazaei; Priscilla Chan; Yao Yao; Zijian Lou; jinil bhavsar; Jian Wang, MD; Michael G. Fehlings, MD, PhD, FRCS(C), FACS

Introduction: Human induced pluripotent stem cell-derived neural stem cell (hiPS-NSC) have the capacity to replace neural circuits, remyelinate denuded axons and provide trophic support making them an exciting regenerative approach after traumatic spinal cord injury (SCI)(1). Unfortunately, most individuals are in the chronic phase of their injury where dense perilesional chondroitin sulfate proteoglycan (CSPG) scarring significantly impairs neurite outgrowth and regenerative cell migration $(2,3)$. Scar-modifying enzymes can enhance NSC-mediated recovery(4); however, nonspecific administration via an intrathecal catheter increases the risk of off-target CNS effects. We aimed to generate a novel, genetically engineered line of hiPS-NSCs, termed Spinal Microenvironment Modifying and Regenerative Therapeutic (SMaRT) cells, capable of locally expressing a scardegrading enzyme to enhance functional recovery without the risk of nonspecific administration.

Methods: A proprietary scar-degrading enzyme was genetically integrated into hiPS-NSCs a SMaRT monoclonal line was generated by single-cell flow sorting. The cells were extensively characterized in vitro. T-cell deficient RNU rats $(\mathrm{N}=60)$ with translationally-relevant chronic C6-7 clip-contusion injuries were randomized to receive: (1) vehicle treatment, (2) hiPS-NSCs, (3) SMaRT cells, or (4) sham surgery (laminectomy alone).

Results: Human SMaRT cells retain key characteristics. The enzyme expressed by SMaRT cells rapidly degrades CSPGs on biochemical assays and allows neurons to extend into CSPG-rich regions in vitro. Furthermore, unlike wild-type hiPS-NSC media, conditioned SMaRT cell media can degrade post-injury rodent CSPGs in ex vivo injured cord cryosections. While blinded neurobehavioural assessments are ongoing with a long-term 32-week endpoint, interim histologic analysis shows that grafted human cells are extending remarkably long $(=20,000 \mu \mathrm{m})$ axons along host white matter tracts from the cervicomedullary junction to the mid-thoracic cord.

Conclusion: This work provides exciting proof-of-concept data that genetically engineered SMaRT cells can degrade CSPGs in vitro and that human NSC transplants can grow long axons in chronic cervical SCI to potentially form a bridge for sensorimotor signal transmission. 
248 Compliance Rates for Cervical Collar Use Following Cervical Spinal Fusion: A Prospective Patient Survey and Feasibility study

Chad Claus, D.O.; Sean D Krieger, BSc; Doris Tong, MD; Sina Rajamand, DO; Teck Mun Soo; Clifford Michael Houseman, DO

Introduction: Postoperative immobilization with external cervical orthosis is a common practice following cervical fusions. Its effectiveness is controversial and compliance data is lacking. We sought to evaluate the effect of cervical collar use on fusion rate and we first evaluated collar use compliance as a feasibility study.

Methods: We prospectively interviewed consecutive patients who underwent anterior/posterior cervical fusion in two hospitals with multiple surgeons at their 2-9 weeks' postoperative visits from $2 / 1-8 / 1 / 2017$. Patients were instructed to wear their cervical collar for at least 23 hours. We excluded personal injuries or patients $<18$-year-old. Patients were interviewed using a cloudbased, secured and encrypted standardized questionnaire (Fig.1) in person or through a phone interview. Patients were considered lost to follow up after 2 phone calls and 1 certified letter. Our primary outcome is self-reported compliance with collar use. Our secondary outcome is patient comfort rating on a 5-point rating scale. Univariate and sensitivity analyses were used. An equivocal compliance rate will indicate feasibility for a second phase randomized controlled trial to evaluate collar use in cervical fusion.

Results: Two hundred thirty-eight $(86.5 \%)$ out of 275 consecutive cervical fusion patients were interviewed. There is no difference between compliant vs. noncompliant patients [Table 1]. One hundred sixty-one $(67.7 \%)$ patients self-reported collar use compliance with $50.4 \%$ wearing for $>23 \mathrm{~h} /$ day (Table 2 and Fig. $2)$. Both compliant and noncompliant patients found collar use uncomfortable (Median 4, $\mathrm{R}=1-5$ ) (Table 3). Sensitivity analysis showed a compliance rate of $45-72 \%$.

Conclusion: About fifty percent of our patients were noncompliant and the median comfort rate was uncomfortable. Our compliance rate demonstrated that collar use might be of questionable effectiveness in achieving fusion. Further investigation is warranted.

\section{Endplate Deformation Due to Open Versus Strutted Intervertebral Devices}

Antonio Valdevit, PhD; Anna Kedzierska; Michelle B Gallagher, MS; Jennifer M Schneider, MS; Peter Ullrich, MD

Introduction: There is a paucity of work on implant/endplate interaction due to implant design to increase fusion rates. The aim of this study was to investigate if bulging of the vertebral endplate is inhibited by implants with a central strut thus limiting graft compression.

Methods: Fourteen porcine (L4, L5) vertebra were assigned to implant groups representing open or strutted devices (Figure1A). A transducer was placed on the endplate surface while $500 \mathrm{~N}$ was applied to the implant at $1 \mathrm{~Hz}$ for 500 cycles (Figure 1B). Load and endplate deformation was acquired at each time point, averaged, and subjected to regression with parameters of; K [or Rate (s-1)], Half-Life (s) and Span [or Compressive Change, (mm)] which were compared using unpaired t-tests.

Results: An elevated Rate (K value) of endplate deformation $(\mathrm{P}<0.01)$ was seen for open implants compared to strutted implants (Figure 2A). Expressing this as Half-Life $(\mathrm{HL}=\ln 2 / \mathrm{K})$ represents the number of cycles or time required to achieve a $50 \%$ change towards settled endplate deformation (Figure 2B). A decreased Half-Life $(\mathrm{P}<0.01)$ was seen for open implants compared to strut- ted implants. The Span parameter represents differences between initial endplate and final endplate deformation (Figure 2C) and displayed an elevated $(\mathrm{P}<0.01)$ increase for open devices compared to strutted implants. Dynamic endplate bulging is represented by the $\mathrm{K}$ value (Rate) and Half-Life data. The results indicate open implants require less time to achieve endplate bulging. The endplate is therefore free to deform, permitting endplate expansion for graft compression. During Compressive Change (Span, Figure $2 \mathrm{C}$ ), open implants provided $15 \mu \mathrm{m}$ of additional expansion. This distance is significant as osteoblasts differentiate at 28 um from capillaries in Static Osteogenesis (SO), while they must move during loading in Dynamic Osteogeneisis (DO).

Conclusion: Open implants permit unimpeded endplate mechanics and may facilitate improved graft compression under cyclic loading.

250 Minimally Invasive Surgery Bertolotti's Syndrome Retrospective Chart Review

Arthur L. Jenkins; Ryan Adams, BS

Introduction: Bertolotti's syndrome is the clinical presentation of chronic lower back pain and/or L5 radiculopathy associated with unilateral or bilateral enlargement of the L5 transverse process and its subsequent articulation, fusion, or partial fusion with the sacral ala or iliac wing. The traditional treatment for patients with Bertolotti's syndrome is an open resection of the transverse process, or an L5-S1 fusion. This research is designed to compare outcomes treated with open reductions of the bony anomaly, fusions of the L5/S1 segment, or with the minimally invasive technique of reductions of the bony anomaly. This chart review analyzed the recovery for patients that have been treated minimally invasive to determine if that approach has advantages over either of the open approaches.

Methods: Patient charts were reviewed from individuals within the PI's practice. Patients were identified by the study team from procedure lists and those that were treated with surgery for Bertolotti's syndrome reviewed. Charts were reviewed in EPIC, the Spine drive, and paper charts for the procedure data as well as the patient's follow up and recovery.

Results: We noted more anatomical variants present under our classification of Bertolottis Syndrome than the established Castellvi criteria. Patients also reported a 35\% decrease in pain post-surgery pertaining to the back and leg pain experienced, although there was some variability on a patient to patient basis.

Conclusion: Identifying the anatomical variants present on a patient to patient basis can allow for more efficient surgical intervention. It was also noted that a minimally invasive surgical approach mitigates several complications that can arise from performing an open procedure and result in better patient outcomes.

251 Stereotactic Navigation for the Pre-psoas Oblique Lateral Lumbar Interbody fusion

Anthony Michael DiGiorgio, DO, MHA; Caleb S Edwards, BA; Michael S. Virk, MD, PhD; Praveen V. Mummaneni, MD; Dean Chou, MD

Introduction: The pre-psoas, retroperitoneal approach is a minimally invasive technique used to perform anterior lumbar interbody fusion. The approach may have a lower morbidity than the trans-psoas approach because it avoids going through the psoas muscle itself by remaining in a plane anterior to it. However, because of the inherent oblique angle to the spine, the approach can be disorienting. Intraoperative navigation resolves this issue.

Methods: A retrospective chart review of patients undergo- 
ing navigated pre-psoas lumbar interbody fusion was performed. Baseline and postop pain scores, OR time, EBL, length of stay and complications were tracked.

Results: 86 levels were fused in 49 patients. The average follow up was 10 months. There was one complication related to the approach a psoas hematoma secondary to oozing but no neurologic, bowel, ureter or major vascular injuries. There were no readmissions within 90 days related to the anterior approach. The average OR time for the surgery with navigation was 183 minutes, which included 3 and 4 level fusions. 20 patients had reached one year of follow up. These patients had significant reductions in VAS back pain (7.17 to $4.10[\mathrm{p}=.001])$ and leg pain (6.15 to 1.20 $[\mathrm{p}<.001])$.

Conclusion: The pre-psoas approach avoids the morbidity of dissecting through the psoas and lumbar plexus. While operative time may be longer than fluoroscopy based lateral procedures, navigation assists with orientation and identifying vital structures as well as taking intraoperative measurements such as interbody graft dimensions.

252 Impact of Mindfulness-based Stress Reduction on Postoperative Patient Reported Pain, Disability, Quality of Life, and Prescription Opioid Use in Adult Degenerative Lumbar Surgery Patients: A Pilot Study

Juneyoung L Yi, MD, MSPH; Christina A Porucznik, PhD, MSPH; Lisa H Gren, PhD; Jian Guan, MD; Andrew T. Dailey, MD; Mark Alexander Mahan, MD; Robert S. Hood, MD; Darrel S. Brodke, MD; Brandon Lawrence; William R Spiker, MD; Nicholas T Spina, MD; Erica Fay Bisson, MD,

Introduction: Prescription opioid medications are a mainstay for pain control after lumbar surgery. However, they negatively impact postoperative outcomes. Furthermore, opioid-related overdose death rates in the United States increased by $200 \%$ between 2000 and 2014. Thus, alternatives are imperative. Mindfulnessbased stress reduction (MBSR) has led to improvements in general activity, mood, walking ability, normal work, and enjoyment of life in opioid-using chronic pain patients. Prospective studies using preoperative MBSR to impact postoperative outcomes in lumbar spine surgery are lacking.

Methods: The intervention group underwent an online MBSR course before surgery. The comparison group was matched twoto-one according to surgical procedure and preoperative opioid use retrospectively. Baseline data collected included demographics, medical history, prescription opioid use, and patient-reported outcomes (PRO's) for pain, disability, and quality of life. Prescription opioid use and frequency of mindfulness use surveys were obtained during the inpatient admission. PRO's and prescription opioid use at 30 days postoperatively were collected. Statistical analyses involved multivariable linear regressions with multiple imputation.

Results: Twenty-five participants were included in the intervention group. Sex, osteoarthritis diagnosis, and PCA use were statistically significantly different between groups. Preoperatively, the intervention group had significantly lower mean VAS-LP, higher mean ODI, and higher mean VAS. Postoperatively, the intervention group had lower mean VAS-BP and higher mean ODI. "mbsr" was a significant predictor of change in VAS-LP $(\mathrm{p}<0.001)$ for all regression models. "mbsr" was also a significant predictor of change in VAS-BP, change in EQ-5D-QALY, and change in MED per day at 30 days for multiple models. "MAAS" was a significant predictor of change in PROMIS-PI for multiple models.

Conclusion: Preoperative MBSR was a significant predictor for change in VASBP, VAS-LP, and EQ-5D-QALY. Frequency of mindfulness use was a significant predictor of change in PROMIS-PI. Further research is needed to asses the effectiveness of preoperative MBSR.

253 Patient-reported Allergies are Associated with Preoperative Psychological Distress and Less Satisfying Patient Experience in a Lumbar Spine Surgery Population

Jay M Levin, BA; Spencer Boyle; Robert Winkelman, BS; Joseph E Tanenbaum, BA; Kalil G. Abdullah, MD; Michael P. Steinmetz, MD; Thomas E. Mroz, MD

Introduction: Patient-reported allergies (PRAs) have been investigated as a predictor of poor patient-reported outcomes following orthopedic surgery. No study has investigated the association between PRAs and the patient experience of spine surgery. We sought to determine whether PRAs are associated with patient experience measured by HCAHPS scores, and clarify the association between PRAs and preoperative anxiety and depression.

Methods: HCAHPS survey data, patient demographics and surgical characteristics were obtained. Allergies were categorized as medical (medications, medical supplies) and non-medical (food, animals). Univariable and multivariable logistic regression models were used to determine whether the number of medical and nonmedical PRAs are associated with HCAHPS scores. Additionally, multivariable logistic regression was used to analyze the association between PRAs and psychological distress, while adjusting for potential confounders.

Results: Of 421 patient included, $66.4 \%$ of patients reported an allergy, with $60.4 \%$ reporting a medical allergy and $17.3 \%$ reporting a non-medical allergy. After adjusting for patient-level covariates, number of medical PRAs was an independent predictor of low satisfaction with communication about a medication's side effects (OR 0.84, $\mathrm{p}=0.049$ ). Number of non-medical PRAs was an independent predictor of low satisfaction with communication about a medication's side effects (OR $0.70, \mathrm{p}=0.046)$, and pain control (OR 0.67, $\mathrm{p}=0.009)$. Additionally, back pain $(\mathrm{p}<0.001)$, decreasing age $(\mathrm{p}=0.001)$ and having a PRA $(\mathrm{p}=0.044)$ were all found to be significantly associated with EQ-5D anxiety/depression. Female gender $(\mathrm{p}=0.048)$ and having a non-medical PRA $(p=0.025)$ were significantly associated with co-existing clinically diagnosed depression.

Conclusion: PRAs were associated with lower HCAHPS scores under several dimensions of the patient experience. Additionally, patients with PRAs were strongly associated with anxiety and depression as measured by both EQ-5D anxiety/ depression and a clinical diagnosis of depression. These findings highlight the potential utility of PRAs to help spine surgeons identify patients at risk for a hospital experience that does not fully meet their needs.

254 BMP vs. Alternatives: Cost Savings and Clinical Outcomes Data in 137 ALIF Patients with 2 yr. Follow-up

Paul J Slosar, MD; Paul J. Holman, MD; James Reynolds; Sarah Martineck; Sydney Slosar; Zain Boghani; William Steele, MD

Introduction: Linking basic science data to clinical results can be difficult. Published studies demonstrate osteogenic properties and low inflammation with micron-textured titanium surfaces, even without the addition of exogenous biologic additives. Interbody implants with these surface characteristics may be able to actively stimulate a portion of the fusion integration, potentially reducing surgeons' reliance on the more expensive and inflammatory biologic additives. 
Methods: 137 patients undergoing anterior lumbar interbody fusions were enrolled consecutively and followed for 24 months. All patients received implants with a unique micron-scale textured surface. Group 1: 75 patients received rhBMP-2. Group 2: 62 patients received a bone graft extender (DBM+AGF or ceramic). Clinical outcomes (VAS/ ODI) were collected for 2 years after surgery. Cost savings analysis was performed using manufacturer's list pricing.

Results: Both groups achieved similar and clinically significant improvements at all time points compared to pre-op baseline. Leg pain was higher at all time points in the BMP cohort (Group 1 ), compared to the non-BMP cohort (Group 2) reaching statistical significance at 2 years. Cost savings between high and low cost biologics was \$2565 (1 level), \$2373 (2 level), and \$2103 (3 level).

Conclusion: Biological activity of bone graft extenders may be less relevant in the presence of an osteogenic fusion implant. There was no measurable clinical benefit realized by using BMP or DBM+GF with this specific implant. The patients in the nonBMP group trended towards better clinical outcomes at all time points with statistically significant lower residual leg pain at 2 years, compared to the BMP group. This study demonstrates that excellent clinical outcomes and significant cost savings can be achieved in these cases without the need for the most expensive or inflammatory biologics.

255 The Effect of Female Body Habitus on Patient Reported Outcomes Following Spinal Intervention

Lara Walsh Massie, MD; Hesham Mostafa Zakaria, MD; Kelly Tundo, BS, CCRP; Lonni Schultz, PhD; Victor W. Chang, MD

Introduction: Positive sagittal balance has consistently been found to be associated with disability. Disproportional breast size in relation to body habitus is known to shift the center of gravity anteriorly and has been demonstrated to increase the thoracic kyphosis and cervical lordosis, which is often correlated with increased pain scores and decreased quality of life. We sought to test the interaction between breast volume and its impact on postoperative outcome in patients undergoing spinal surgery.

Methods: Our surgical database identified 175 female patients undergoing spinal surgery within the past two years who also had mammographic images available for review. Breast volume was calculated from mammograms. Patient reported outcomes (PRO) included pre- and 1-year post-operative Oswestry/Neck Disability Index(ODI/NDI), and Visual Analog (VAS) Neck/Back and Arm/ Leg pain score data.

Results: The mean age was 61.6 years, $141(81 \%)$ had lumbar surgery, $34(19 \%)$ underwent cervical surgery, $115(66 \%)$ had a fusion procedure and $74(43 \%)$ were single level. The average BMI was $31.6 \pm 6.7$ and the average breast size was $1350.1 \pm$ 686.1cc. The average baseline PROs were 51.3 for ODI/NDI, 7.4 for back/neck pain and 7 for leg/arm pain. There was no significant association between absolute breast volume and pre-operative disability or pain scores. Change in PRO scoring at 1 year was significantly correlated with breast volume (ODI/NDI $\mathrm{r}=-0.181$, $\mathrm{p}=0.024 ;$ Back/Neck $\mathrm{r}=-0.209, \mathrm{p}=0.01$ and Leg/Arm $\mathrm{r}=-0.23$, $\mathrm{p}=0.004)$. When stratifying breast volume into thirds, the tertile with the lowest breast volume had significantly higher rates of minimal clinically important difference (MCID) for ODI/NDI than the highest tertile $(68 \%$ vs $45 \%, \mathrm{p}=0.019)$ and extremity pain $(85 \%$ vs $63 \%, \mathrm{p}=0.033$ ).

Conclusion: While breast volume is not predictive of preoperative pain and disability scores, it has a significant correlation with the improvement in disability, axial, and extremity pain following spinal surgery and should be considered in pre-surgical counseling.
256 Should Physical Therapy be Incorporated in Patient Care Post 1-2 Level Lumbar Fusions for Degenerative Lumbar Instability? - A Comparative Outcome Analysis

Devender Singh, PhD; Eeric Truumees, MD; Marcella A. Madera, MD; Trent McGinty, DPT; Jeremy Brady, DPT

Introduction: Fewer studies in the literature have examined the role of post-operative physical therapy (PT) after lumbar fusion. In the post-operative setting, much of the rehabilitation literature focuses on post-discectomy patients. The study aims to evaluate and compare the changes in pain and functional scores between patients who completed PT versus no-PT after their anterior or posterior/posterolateral 1-2 level lumbar fusions for degenerative lumbar instability.

Methods: We assembled a clinical database of patients with PT and no-PT visits post anterior or posterior/posterolateral 1 or 2 levels lumbar fusion surgery for degenerative disease from June 1st, 2014-April 30th 2016. Basic demographic and outcome scores such as Oswestry disability index (ODI) and Visual analog scale (VAS) were collected. Independent two-sample with unequal variances were used to assess for differences between the two groups $(\mathrm{a}=0.05)$.

Results: 72 and 51 patients in PT and no-PT met the inclusion criteria, respectively. There were higher numbers of females $(63.8 \%)$ in PT group. No-PT group had similar numbers of males and females. Mean ages for PT and no-PT groups were $61.4( \pm 14.7)$ and $59.5( \pm 14.1)$ years, respectively. Both groups were similar with respective to body mass index. Majority of the cases in both groups involved posterior/posterolateral approach. $55.5 \%$ of patients in PT group and 50.9\% in no-PT had 1 level lumbar fusion. Mean change (pre-operative vs. 1 year post-operative) in ODI between the two groups differed significantly (PT vs. no-PT: $18.8 \pm 13.9$ and $9.3 \pm 17.5 ; \mathrm{p}=0.019$ ). Similarly, mean change in VAS was significantly different between the two groups (PT vs. no-PT: $3.2 \pm 2.18$ and $1.05 \pm 2.08 ; \mathrm{p}=0.026$ ). None of our PT patients reported any change in pre vs. 1 year post-operative follow up employment status (35\% retired, $38 \%$ employed fulltime, $11 \%$ unemployed and $16 \%$ homemaker). $98 \%$ of No-PT patients reported no change in employment status during the same timeframe (46\% retired, $25 \%$ employed fulltime, $13 \%$ unemployed and $15 \%$ homemaker).

Conclusion: PT group reported significantly positive changes in functional and pain scores than their counterparts. We conclude that post-lumbar fusion rehabilitation may benefit majority of the adult patient population.

257 Nonsurgical Management of Occipital Condyle Fractures

\section{Adam A Khan, MD; Molly E. Hubbard, MD}

Introduction: Occipital condyle fractures are rare injuries in the cervical spine. Initial studies recommended treatment with a rigid cervical collar for types I and II fractures, and surgical fixation for "unstable" or type III fractures. The purpose of this study is to review our experience with non operative management of these fractures at a level 1 trauma center.

Methods: A retrospective review of 1280 trauma patients to a level one trauma center was performed over a 10 year period. There were 108 patients identified with occipital condyle fractures, 37 of them bilateral. Demographic information, additional levels of cervical spine fractures, fracture characteristics according to both major classification systems, management (halo vs collar) and follow up at 1 month, 3 months and 1 year (if available) were 
recorded. Follow up cervical spine CT scans and $\mathrm{X}$ rays were reviewed for all patients for which imaging is was available.

Results: 108 patients were identified. 18 were treated surgically. 90 were treated conservatively without surgical intervention. 27 were managed with a halo; 63 were manage with surgery; none required eventual surgical management.

Conclusion: Multiple grading systems have been proposed for classification of these fractures, however our experience is that regardless of the fracture type, non operative management for those without atlantoaxial dissociation is a successful treatment protocol.

258 Surgical Management of Unstable Traumatic Upper Thoracic (T1-T6) Fractures: Prospective Case Series.

\section{Domenico A Gattozzi, MD; Paul M. Arnold, MD}

Introduction: Traumatic upper thoracic spine fractures (T1T6) have unique anatomic features. These injuries are frequently associated with complete spinal cord injury and multiple other traumatic injuries, and surgical management is often challenging. We present our experience in managing these fractures.

Methods: We analyzed a prospectively collected series of unstable, traumatic, upper thoracic (T1-T6) spine fractures treated between 1993 and 2016. Preoperative evaluation included neurological exam and radiographic analysis. Indications for surgery were spinal instability and neurological deficit. Clinical and radiographic outcomes included neurological status, fusion rates, hospital length of stay, complication rates, and successful fixation of the fracture.

Results: Forty-three patients (29 male, 14 female) were included. Mean age was 37.7 years (range 14-83 years). There were 18 burst fractures, 13 fracture dislocations, and 12 compression fractures. There were 32 motor vehicle collisions, 9 falls, one sports-related injury, and one injury from a falling object. From 1993-1999, eight patients were treated with hook and rod constructs. From 1995-2016, thirty-five patients were treated with pedicle screw fixation. There were 86 total levels fixed. There were no intraoperative vascular or neurologic complications. Fusion rate was $95 \%$. At presentation there were 26 ASIA-A, 3 ASIA-B, 3 ASIA-C, 4 ASIA-D, and 7 ASIA-E patients. At final follow-up there were 14 ASIA-A, 7 ASIA-B, 1 ASIA-C, 1 ASIA-D, and 10 ASIA-E patients. All patients were followed for at least one year. No patient was neurologically worse, and hardware was removed post-fusion in five patients due to pain, wound infection, or hardware failure. Mean hospital length of stay was 21.1 days (range 4-71 days).

Conclusion: Surgical treatment of upper thoracic spine fractures with pedicle screws is safe and effective. Reduction and fixation of these fractures results in reduced risk of further neurological complications and allows for earlier mobilization, which correlates with shorter hospital lengths of stay and improved patient outcomes.

259 Comparative Analysis of Radiologic Outcomes of C1-2 Fusion Spine Surgery Between Intraoperative CT ImageBbased Navigation Guided Operation and Fluoroscopy Guided Operation

Dong Wuk Son; Geun Sung Song; Sang Weon Lee; Jun Seok Lee, M.D.

Introduction: Fixation of $\mathrm{C} 1-2$ is challenging surgical procedure because of the complex anatomy and the need for a high degree of accuracy to avoid complication. Intraoperative 3D image $(\mathrm{O}$-arm) based navigation system reduced complications, such as vertebral artery injury. In this study, we compared the surgical outcomes during the cervical fixation with and without intraoperative navigation.

Methods: We retrospectively reviewed radiologic images of 26 patients who underwent $\mathrm{C} 1-2$ fusion spine surgery from January 2009 to November 2016 at our institution. There were 11 degenerative cervical disease and 14 traumatic cases. A total 107 screws were used and the surgical procedures were as follows; C1 lateral mass screw $(\mathrm{C}-\mathrm{arm}: \mathrm{n}=30, \mathrm{O}-\mathrm{arm}: \mathrm{n}=22), \mathrm{C} 2$ pedicle screw(C-arm: n=26, O-arm: $n=21), C 2$ lamina screw $(C$-arm:n=2, O-arm:n=1), 5 short pars screw screws were placed under a c-arm guide. Accuracy of screw position was determined as following; Grade 0 : screw inside the bone, perforation of the cortex by(Grade I: $<2 \mathrm{~mm}$, Grade II:from 2 to $4 \mathrm{~mm}$, Grade III : $>4 \mathrm{~mm})$. We also classified screw malposition as grade 0,1 : minor malposition, and grades 2,3: major malposition.

Results: The results of accuracy of screws positioning was summarized on table 1 . In 1 cases, we confirmed the malposition of $\mathrm{C} 2$ pedicle screw and corrected the screw positon. There was no complications regarding vertebral artery injury and no new onset neurologic deficit developed in any of the patients.In two group, Accuracy of the screw position was acceptable, but there was no statistical significance. The operating time, EBL, fusion rate, also does not show statistical significance. (Table 2)

Conclusion: In two group, Accuracy of the screw position was acceptable.With intraoperative $\mathrm{O}$-arm system, we could confirm the misposition of screw, and could correct the position of screw in the operation

\section{Occipital Condyle Fractures: Is a Collar Necessary?} A Pilot Study MD

James Leonard West, MD; Lukas Vilella; Stacey Q. Wolfe,

Introduction: Occipital condyle fractures (OCFs) have previously been described based upon anatomic characteristics of the fracture; however, recent literature 1 has proposed management based on biomechanical stability. The treatment of biomechanically stable fractures varies from observation to the use of cervical collar. Prior to determining best management, understanding of concomitant cervical spine fractures in the presence of OCFs is prudent. The primary aim of this pilot study was to determine the rate of occurrence of biomechanically significant cervical spine fractures with OCFs.

Methods: A retrospective chart review of all leveled trauma patients presenting to Wake Forest Baptist Health from 2013 to 2017 with the diagnosis of occipital condyle fracture.

Results: There were 46 total patients who presented with OCF, with an average GCS 12 upon presentation and average Injury Severity Score of 23. Of these patients, the average age was 42.1 years and 4 patients were found to have bilateral OCFs. Approximately $30 \%$ of these patients had associated intracranial injuries, and $59 \%$ had an associated cervical spine injury. The overall rate of associated biomechanically significant fracture associated with an OCF was $43.5 \%$. Treatment of OCF included collar immobilization (83\%) and observation (17\%). Average follow up was 3.37 months. There were no significant neurologic injuries or cranial nerve palsies associated with these fractures in either group.

Conclusion: This study better characterizes the cervical spine fractures which occur concomitantly with OCFs. The results indicate that the majority of patients with occipital condyle fractures do not have biomechanically significant fractures elsewhere in the cervical spine. This subset of patients will be the cohort for a follow-up randomized controlled study to assess if cervical collar immobilization is necessary in treatment of OCFs. 
261 Does the Coexistence of Multiple Segmental Rib Fractures in Polytrauma Patients Presenting with "Major" Vertebral Fracture Affect Care and Acute Outcomes?

Joseph Robert Kapurch, MD; Mohammed Ali Alvi, MD; David V. Ivanov; Panagiotis Kerezoudis; Sandy Goncalves; Brett Arthur Freedman; Mohamad Bydon, MD

Introduction: Patients with concomitant segmental rib fractures/flail segments and unstable vertebral fractures requiring surgical fixation pose an additional operative challenge due to the prone positioning required for access to the posterior spine. Herein, we sought to determine if operating on "major" vertebral fractures leads to premature abortion of surgery and/or other acute cardiopulmonary complications.

Methods: We retrospectively queried our institutional Trauma Registry, which is a prospectively maintained database since 1990 for all cases presenting with concomitant segmental rib fractures and Denis "major" vertebral fracture where the vertebral fractures were surgically managed. Variables of interest were extracted and were analyzed. Additionally, we performed a case control analysis to compare the outcomes of these patients with those without rib fractures.

Results: We found 57 cases with concomitant rib fractures and vertebral fractures where the latter were surgically fixed. $80.70 \%$ patients $(n=46)$ required ICU admissions with a median ICU length of stay (LOS) of 3 days $(\mathrm{IQR}=0-9), 38 \%(\mathrm{n}=66)$ required ventilator assistance with median duration of 5 days $(\mathrm{IQR}=2-9.5)$. Hospital LOS was found to be 12 days (IQR= 8-19). $34 \%$ patients $(n=19)$ were discharged to home, $28.57 \%(n=16)$ each to nursing house and rehab facility. $30.36 \%$ of the patients $(n=17)$ were discharged with some form of brace. Additionally, 4 vertebral fixation cases $(7.02 \%)$ had to be aborted due to intra-operative cardiopulmonary complications, of which 1 expired due to ICP complications. On propensity-score-matched case-control analysis, clinical outcomes of patients with concomitant rib fractures and vertebral fractures were found to be similar to those without rib fractures.

Conclusion: Our findings suggest that it is appropriate to consider rib fixation prior to repeat spine surgery, although delaying surgery and allowing underlying pulmonary contusions and other acute injuries to further stabilize may be an alternative effective option.

262 Contrast-enhanced Ultrasound to Visualize and Quantify Local Blood Flow and Perfusion After Traumatic Spinal Cord Injury

Zin Khaing; Matthew Bruce; Lynn B McGrath, MD; Lindsay Cates; Christoph Paul Hofstetter, MD, PhD

Introduction: Traumatic spinal cord injury (tSCI) causes an almost complete loss of blood flow at the site of injury (primary injury), as well as significant hypoperfusion in the penumbra of the injury. Hypoperfusion constitutes a major contributor to progressive cell death of spinal cord tissue that was initially viable (secondary injury). Neuroprotective treatment strategies seek to limit secondary injury. Clinical monitoring of the temporal and spatial patterns of blood flow within the contused spinal cord is currently not feasible.

Methods: We developed ultrafast contrast enhanced ultrasound (CEUS) Doppler utilizing a research ultrasound platform (Verasonics Vantage, USA) combined with a $18 \mathrm{MHz}$ linear array transducer (Vermon, France). High frequency ultrasound enabled visualization of spinal cord vessels with a diameter ranging from
20 to $400 \mu \mathrm{m}$. Ultrafast plane wave acquisitions ( $>30,000$ frames/ second) enable, for the first time, the separation of higher velocity flow signals of larger vasculature from low velocity blood flow within the microcirculation. Rat spinal cords were imaged prior and after a compression injury of the thoracic spinal cord

Results: In the intact spinal cord, ultrafast CEUS Doppler confirmed higher perfusion of the gray matter compared to white matter. Immediately after a compression injury of the thoracic rodent spinal cord, spinal cord vessels are disrupted in an area of $1.931 .14 \mathrm{~mm}$. Ultrafast CEUS Doppler revealed a topographical map of local tissue hypoperfusion with remarkable spatial resolution. Critical loss of perfusion, defined as less than $40 \%$ perfusion compared to the surrounding spared tissue, was seen within an area of $2.210 .6 \mathrm{~mm} 2$.

Conclusion: In our current report, we introduce ultrafast CEUS doppler for monitoring of spinal vascular structure and function in real-time. Development and implementation of this type of imaging in the clinic could have significant impact on management and care of patients with spinal cord injury.

263 Natural History of Neurological Improvement Following Complete (AIS A) Thoracic Spinal Cord Injury Across Available Registries to Guide Acute Clinical Trial Design and Interpretation

James D. Guest, MD, PhD; Steven Kirshblum, MD; Armin Curt; Trey Mobley; Robert G. Grossman, MD; Alex Aimetti, PhD

Introduction: Investigational therapies for acute spinal cord injury (SCI) are typically initiated in neurologically complete (ASIA Impairment Scale \{AIS $\}$ A), thoracic injured patients as the risk for neurological deterioration is low. Therefore, a robust characterization of the natural history of neurological improvement following clinical trial-eligible thoracic AIS A injuries is needed for efficient study design and interpretation of results. Here we present for the first time a compilation of neurological recovery data from three established SCI registries: North American Clinical Trials Network (NACTN), European Multicenter Study about Spinal Cord Injury (EMSCI), and Spinal Cord Injury Model Systems (SCIMS).

Methods: Only patients injured between 2006-2016 and meeting common acute thoracic AIS A clinical trial inclusion/exclusion criteria such as age 16-70, T2-T12 neurological level of injury (NLI), AIS A, non-penetrating injury, acute neurological exam within 7 days of injury, and follow-up neurological exam at least $\sim 6$ months post-injury, were included in this analysis. International Standards for Neurological Classification of Spinal Cord injury outcomes including AIS conversion rate, NLI, and sensory and motor scores/levels were compiled.

Results: A total of 169 patients were included from the three registries: 11 from NACTN, 64 from EMSCI, and 94 from SCIMS. AIS conversion rates at approximately 6 months postinjury varied from $18.2 \%-23.4 \%$. Improved conversion rates were observed in all registries for low thoracic (T10-T12) injuries when compared to high/mid thoracic (T2-T9) injuries. NLI was consistently stable and lower extremity motor score (LEMS) improvement was uncommon.

Conclusion: This study presents the aggregation of multinational natural history recovery data in thoracic AIS patients from three SCI registries and demonstrates that neurological improvement following these injuries is minimal. This work aims to guide the development of future clinical trial protocols as well as aid in the interpretation of the safety and potential clinical benefit of new therapies. 


\section{Posterior-only Stabilization for Traumatic Thoracolumbar Burst Fractures}

Omid R. Hariri, D.O., MSc.; Ariel Takayanagi, BA; Hammad Ghanchi, DO; Quang Ma; Dan Miulli, DO, FACOS

Introduction: No consensus exists for the management of unstable thoracolumbar (TL) burst fractures. Surgical options include anterior, lateral, or posterior stabilization (or some combination of those options), depending on the fracture. The potential benefits of anterior reconstruction (eg, direct compression, better kyphotic deformity correction, and 3-column stability) come with increased operative time and associated morbidity. A posterioronly approach can offer stable correction without increased operative risks, but may increase the risk of limited kyphotic correction. Anterior stabilization may offer better decompression and neurological improvement compared to posterior treated patients. The goal of this study was to determine whether posterior-only stabilization is a viable treatment option for patients with traumatic TL fractures as opposed to anterior and combined approaches.

Methods: We performed a retrospective analysis of adult patients with TL burst fractures who underwent posterior-only surgical intervention from 2005 to 2015. Operations were performed at 2 levels above and below the fractured segment using pedicle screw-rod fixation constructs with autograft and allograft. All patients received TL bracing for at least 3 months. Patients lost to follow-up were excluded.

Results: Sixty-four consecutive patients with posterior-only stabilization were identified, with 18 lost to follow-up. Of the remaining 46 patients, $93 \%$ were male, and $7 \%$ were female, with a mean age of 36.8 years. Mean time until removal of the brace was 3.54 months. No patients required additional surgical intervention for spinal stabilization. Three patients experienced postoperative complications, all of which were related to infection.

Conclusion: Our data indicate posterior-only stabilization for traumatic TL burst fractures is a durable and effective option in select patients. The approach offers surgical intervention with a decreased peri-operative risk as well as morbidity and mortality, with a minimal increase in the risk of kyphotic deformity. Further prospective studies are necessary to validate these findings clinically.

265 Diffusion Tensor Imaging Evaluation of Cervical Spinal Cord Injury

Nikolay L. Martirosyan, MD; Jennfer Becker; Elizabeth Krupinski; William Erly; Wayne Kubal; R. John Hurlbert, MD, $\mathrm{PhD}, \mathrm{FACS}, \mathrm{FRCS}(\mathrm{C})$

Introduction: Contusive spinal cord injury is a life-altering condition affecting primarily young people, resulting in varying degrees of paralysis. Novel therapies are being developed that show promise in mitigating the disability attendant with this condition. Accordingly, it is important to develop reliable, reproducible methods to quantitatively assess spinal cord integrity not only at the time of injury but in follow-up afterward. The purpose of this study was to evaluate DTI as a tool to establish axonal integrity in the clinical setting of acute cervical spinal cord injury.

Methods: Diffusion tensor imaging was performed in normal volunteers $(n=10)$, patients with acute cervical spine fracture $(n=35)$, and patients with acute spinal cord injury $(n=5)$ within 24 hours of injury. Imaging was performed on a 3T Siemens Skyra Scanner, (Siemens, Erlangen, Germany). The imaging protocol utilized monopolar DTI employing a 3D radial vibe technique with fat saturation (bandwidth 2004, echo spacing 0.58, ET 61, TR 3500 , slice thickness $1.5 \mathrm{~mm}$, no gap, 32 sagittal slices, phase direction A-P, b value 600, b1: 4 averages, b2: 7 averages). Postprocessing was performed using Neuro 3D, Syngo.Via VB30 (Siemens Heathineers, Malvern PA). Diffusion tensor imaging results were correlated with neurological assessment and ASIA score.

Results: High quality DTI data was obtained in all subjects. Data from 10 volunteers correlated precisely with the 35 fracture patients without neurologic deficit. Tensor values (fraction anisotropy) were significantly reduced for patients with spinal cord injury. Fractional anisotropy varied in a statistically significant manner as a function of level of injury and ASIA grade.

Conclusion: Diffusion tensor imaging reliably detects acute spinal cord injury within 24 hours of trauma. The technique is precise, reproducible and robust. Changes in fraction anisotropy correlate with the degree of neurological deficit.

266 Perioperative Thromboembolism and Longer Construct Length are Associated with Wound Reoperation in Patients with Vertebral Metastases

Zachary Pennington; Hannah Carl; Rafael De la Garza-Ramos, BA; Nancy A Abu-Bonsrah, MD; A. Karim Ahmed; Eric W. Sankey, MD; C. Rory Goodwin, MD, PhD; Ali Bydon, MD; Timothy F. Witham, BS, MD; Jean-Paul Wolinsky, MD; Ziya L. Gokaslan, MD; Justin Sacks, MD; Daniel M

Introduction: Operative management is often performed on patients with vertebral metastases to relieve pain, stabilize the spine, and improve neurologic function. Previous literature has shown that $10-52 \%$ of these operations will result in complications, the most common of which is wound infections, which occurs in $4-20 \%$ of cases. These infections lengthen hospital stays, increase mortality, and raise the cost of care by upwards of $60 \%$, with wound revisions account for $38 \%$ of the additional costs. The objective of this study is to determine which perioperative risk factors correlate with the need for wound revision in patients treated for vertebral metastases

Methods: A single-institution retrospective review of all patients operated for vertebral metastases between 2003 and 2013 was performed. Factors with a p-value $<0.200$ on univariable analysis were included in the multivariable model.

Results: Our series included 159 patients $(53.5 \%$ male, mean 59.6y.o.). Factors included in the multivariable analysis were preoperative hypertension, hyperlipidemia, number of treated levels, smoking status, Karnofsky Performance Status $>70 \%$, the occurrence of perioperative venous thromboembolism (VTE), and use of a posterior approach. The number of treated levels and occurrence of thromboembolic events (95\% CI: 1.19-48.5; p-value: 0.032 ) were both independently associated with wound reoperation in the multivariable model. Each additional treated level was associated with a $21 \%$ increase in risk of wound reoperation $(95 \%$ CI: $1.03-1.43, \mathrm{p}=0.018)$.

Conclusion: All patients operated for vertebral metastases are at increased of wound infection and subsequent reoperation owing to receipt of adjuvant radiotherapy and other medical comorbidities. However, our results suggest that patients experiencing perioperative VTE and those with larger incisions are at the highest risk. As a result, interventions designed to reduce VTE and improve wound care may reduce the frequency of revision surgeries and reduce associated costs. 
267 Functional Outcome after Thoracic Spinal Cord Injury: Analysis of Risk Factors in the NACTN Longitudinal Prospective Cohort Study

Blessing N.R Jaja; Jefferson R. Wilson, MD, PhD; Mario Ganau, MD, PhD, FACS; Michael G. Fehlings, MD, PhD, FRCS(C), FACS

Introduction: Thoracic-level spinal cord injuries (tSCI) have unique biomechanical and physiological characteristics and their relative incidence is high (second only to cervical-level injuries). This study investigated functional outcomes at one-year and the associated risk factors in a cohort of thoracic SCI from the NACTN longitudinal prospective study.

Methods: Functional outcomes were assessed according to the Spinal Cord Independence Measure (SCIM) total score and the sub-scores for ambulation, bladder, and bowel management. Descriptive statistics and regression analysis were applied to examine the effect of clinical and treatment variables on the outcomes.

Results: Among the 68 patients who were assessed at one-year follow-up, 13 (19\%) could ambulate without support, 33 (49\%) had normal bowel function, and $25(37 \%)$ had normal bladder function. The rates did not differ with respect to patients age, gender, mechanism of injury (penetrating versus non-penetrating), premorbid health status, time to surgical decompression, injury levels, and incidence of complication. Patients with incomplete injury (baseline AIS grade B/D) were more likely to independently ambulate, have normal bladder and bowel function at one-year, compared to patients with complete injury (grade A); $p$ value < 0.001 . The Area under the receiver operator characteristics curve associated with baseline AIS grade was 0.75 for ambulation and for bowel function, and 0.88 for bladder function.

Conclusion: Patient baseline AIS grade, as a standalone predictor, satisfactorily differentiated between those who did or did not ambulate, have normal bowel or bladder function at one-year. It is the primary prognostic factor for long term functional outcomes following traumatic thoracic SCI.

268 Intra-operative Computed Tomography (O-ARM) Guided Anterior Odontoid Screw Fixation in Odontoid Fractures

Dattaraj Sawarkar; Pankaj Kumar Singh; Deepak Agrawal, MBBS, MS, MCh; Deepak Kumar Gupta PhD; G.D. Satyarthee; P. Sarat Chandra; Shashank Sharad Kale, MBBS, MS, MCh, MD; Ashok Kumar Mahapatra, MD

Introduction: Anterior OS fixation allows the physiological treatment with preservation of C1-C2 motion. Accuracy of screw placement is one of the important factors necessary for adequate fusion. Various series have reported screw malposition from 0 to $27.2 \%$. The purpose of this study is to evaluate the intra-operative computed tomography (O-arm) assistance for accurate insertion of an anterior odontoid screw (OS) and the ultimate outcome.

Methods: Surgery consisted of anterior OS fixation under intra operative CT (O-arm) assistance (April,2010- April,2015). Demographical, clinical, radiological, perioperative details were retrieved from hospital database. American Spinal Injury Association (ASIA) impairment scale and bony fusion rate were assessed to evaluate outcome.

Results: 50 patients (Mean age 34.6 years, SD 14.10, range: 7- 70 years; 44 males and 6 females) with Type II and Type III odontoid fracture underwent $\mathrm{O}$-arm assisted anterior OS insertion. Mean operating time was $132.2 \mathrm{~min} \pm \mathrm{SD} 33.56$ with average blood loss of $93 \mathrm{ml} . \pm$ SD61.46. With our technique, accurate screw placement was achieved in $100 \%$ patients. At the mean follow up of 26.4 month, overall fusion rate was $97.8 \%$ with nonunion in $2.2 \%$ patients. Morbidity occurred in two patients; one patient developed fixation failure while other had nonunion requiring posterior $\mathrm{C} 1-\mathrm{C} 2$ arthrodesis. Surgical mortality occurred in one patient due to $\mathrm{SAH}$.

Conclusion: We conclude that use of $\mathrm{O}$-arm with acquisition intraoperative three-dimensional CT for anterior odontoid screw fixation adds precision as well as increase the success of surgical techniques by increasing accuracy of anterior odontoid screw placement. It further enable us to extend the indications of odontoid screw fixation to selected complex Type II and rostral Type III odontoid fractures and there for allowing us to do the more physiological neck motion preserving surgery in these subsets of patients.

269 Venothrombolic Events Following Spinal Fractures: A Single Center Experience

Michael Cloney, MD; Jonathan Tad Yamaguchi, BS; Benjamin Hopkins, BS; Zachary Adam Smith, MD; Tyler R. Koski, MD; Nader S. Dahdaleh, MD

Introduction: Venous thromboembolic events (VTE), including both deep venous thrombosis (DVT) and pulmonary embolism $(\mathrm{PE})$, are a major cause of readmission, morbidity, and mortality after spine surgery. Patients with spinal fractures are particularly at an increased risk for VTE after spine surgery. Therefore, understanding VTE risk factors in this patient population is critical. Here, we examined factors associated with venous thromboembolic events among patients with spinal fractures.

Methods: We retrospectively examined records from 195 consecutive patients with spinal fractures who underwent spinal stabilization surgeries- amongst a cohort of 6869 patients who underwent spinal surgery. We collected data on patient demographics, surgery, hospital course, and 30-day rates of VTE, readmission, reoperation. Multivariable logistic regression was used to identify independent predictors of each outcome.

Results: Among 195 patients undergoing surgery for spinal fractures, 18 (9.2\%) experienced a VTE, compared to $2.3 \%$ among all other spine patients (OR 4.466 [2.679, 7.444], $\mathrm{p}<0.0001)$. Ninety-five spine fracture patients $(48.7 \%)$ received chemoprophylactic anticoagulation, compared to $35.7 \%$ of all other spine patients (OR 2.657 [1.996, 3.537], $\mathrm{p}<0.0001)$. EBL was associated with VTE within 30 days of surgery (OR 1.001 [1.000, 1.002], $\mathrm{p}=0.0415$ ) and with DVT within 30 days of surgery (OR 1.001 $[1.000,1.002], \mathrm{p}=0.049)$, and comorbid cardiac disease burden showed a trend toward significance in predicting both VTE (OR $1.890[0.894,3.994], \mathrm{p}=0.0956)$ and DVT (OR 4.228 [0.970, 18.435], $\mathrm{p}=0.0549)$. Number of levels in surgery predicted PE within 30 days of surgery (OR 1.573 [1.111, 2.227], $\mathrm{p}=0.0107$ ).

Conclusion: Compared to all other patients undergoing spine surgery, patients with spinal fractures are more likely to receive chemoprophylactic anticoagulation, but nevertheless have a higher rate of VTE events. EBL and comorbid disease burden predict VTE events in patients with spine fractures

270 Patterns of Neurological Deficit and Recovery of Postoperative C5 Nerve Palsy

\section{John Kenneth Houten, MD; Colin Niezgoda, PAC}

Introduction: C5 nerve paresis is a well-recognized complication of cervical spine surgery. Numerous studies have investigated incidence and possible causes, but few detail the specific pattern of neurological deficit and recovery. 
Methods: Records of patients undergoing cervical decompressive surgery including the $\mathrm{C} 4 / 5$ level were retrospectively reviewed over a 14-year period, identifying cases complicated by C5 nerve paresis. In addition to demographic/clinical data, narrative descriptions of the appearance and course of neurological changes were noted. Minimum follow up was 12 months.

Results: Of 462 patients, 15 developed C5 palsy (2.4\%), 6/303 following anterior surgery $(1.9 \%)$ and $9 / 159$ following cervical laminectomy and fusion $(5.7 \%)$. There were nine men and six women mean age 66.6 (54-74). All presented with deltoid weakness, but $80 \%$ also demonstrated bicep weakness, $40 \%$ tricep, and one in hand intrinsics. Mean onset of motor deficit was 4.9 days (2-14). Six (40\%) complained of shoulder pain but only three $(20 \%)$ had detectable sensory loss. In eleven (73\%), maximal weakness was noted abruptly; but four patients $(27 \%)$ had weakness gradually progress to maximal deficit over 48 hours. Weakness began to improve at a mean 12.3 weeks (4-50); and $87 \%$ had recovery at least to $4 / 5$ in the deltoid. There was no instance of intraoperative loss of somatosensory or motor evoked potentials, nor was any sustained or abnormal intraoperative C5 EMG activity noted in any patient. Patient data is summarized on Table 1.

Conclusion: The pattern of C5 palsy is variable, but patients was characterized in all by delayed onset, mean 4.9 days post surgery, and frequent weakness of muscles beside the deltoid, particularly the bicep. Deltoid muscle recovery to at least $4 / 5$ typically occurs.. Pain and cutaneous sensory loss occurred in a minority of patients. Neuromonitoring was not predictive of deficit in any patient.

271 Worldwide Steroid Prescription for Acute Spinal Cord Injury

Asdrubal Falavigna, PhD; Francine W Quadros; Alisson R Teles, MD; Chung Chek Wong; Giuseppe Barbagallo, MD; Darrel S. Brodke, MD; Abdulaziz Al-Mutair; K Daniel Riew, MD

Introduction: There has always been great interest in discovering effective treatments for spinal cord injury (SCI), since it is a debilitating pathology with considerable socioeconomic consequences. Over the years, publications involving the use of methylprednisolone (MP) after acute SCI have described conflicting results. While some papers demonstrated efficacy others showed no benefits. The worldwide overview on the use of MP, its reasons, and beliefs of different regions will provide a guide for educational activities.

Methods: English version of a previously published questionnaire were used to evaluate opinions about MP administration in acute SCI in LA, EU, AP, NA, and ME. This internet-based survey was conducted by members of AOSpine. The questionnaire asked about demographic features, background with management of spine trauma patients, routine administration of MP in acute SCI and reasons for MP administration.

Results: A total of 2,659 responses were obtained for the electronic questionnaire from LA, EU, AP, NA, and ME. The number of spine surgeons that treat SCI was 2,206 (83\%). The steroid was used by 1,198 (52.9\%) surgeons. The uses of MP were based predominantly on the National Acute Spinal Cord Injury Study (NASCIS) III study $(\mathrm{n}=595,50 \%)$. The answers were most frequently given by spine surgeons from AP, ME, and LA. These regions presented a statistically significant difference from North America $(p<0.001)$. The number of SCI patients treated per year inversely influenced the use of MP. The higher the number of patients treated, the lower the administration rates of MP were observed.

Conclusion: The study identified potential targets for educational campaigns, aiming to reduce inappropriate practices of MP administration.
272 Rehabilitation of Hand Function After Spinal Cord Injury Using a Novel Handgrip Device: A Pilot Study.

Tianyi Niu, MD; Daniel C. Lu, MD, PhD; Yevgeniy Freyvert, $\mathrm{MD}$; Nicholas Au Yong, MD, PhD

Introduction: Activity-based therapy (ABT) for patients with spinal cord injury (SCI), which consists of repetitive use of muscles above and below the spinal lesion, improves locomotion and arm strength. Less data has been published regarding its effects on hand function. We sought to evaluate the effects of a weekly hand-focused therapy program using a novel handgrip device on grip strength and hand function in a SCI cohort.

Methods: Patients with SCI were enrolled in a weekly program that involved activities with the MediSens (Los Angeles, CA) handgrip. These included maximum voluntary contraction (MVC) and a tracking task that required each subject to adjust his/ her grip strength according to a pattern displayed on a computer screen. For the latter, performance was measured as mean absolute accuracy (MAA). The Spinal Cord Independence Measure (SCIM) was used to measure each subject's independence prior to and after therapy.

Results: Seventeen patients completed the program with average participation duration of 21.3 weeks. The cohort included patients with American Spinal Injury Association (ASIA) Impairment Scale (AIS) A $(n ?=? 12)$, AIS B $(n ?=? 1)$, AIS C $(\mathrm{n} ?=? 2)$, and AIS D $(\mathrm{n} ?=? 2)$ injuries. The average MVC for the cohort increased from $4.1 \mathrm{~N}$ to $21.2 \mathrm{~N}$ over 20 weeks, but did not reach statistical significance. The average MAA for the cohort increased from 9.01 to $21.7 \%$ at the end of the study (p?=?.02). The cohort's average SCIM at the end of the study was unchanged compared to baseline.

Conclusion: A weekly handgrip-based ABT program is feasible and efficacious at increasing hand task performance in subjects with SCI.

273 Early Chemical DVT Prophylaxis After SCI is Safe and Effective: Pilot Prospective Data

Anthony Michael DiGiorgio, DO, MHA; Rachel Tsolinas; Mohanad Alazzeh, BS; Jenny Haefeli; Jason Talbott, MD, PhD; Adam Ferguson, PhD; Jacqueline Bresnahan, PhD; Michael Beattie, PhD; Geoffrey T. Manley, MD, PhD; William Whetstone, MD; Praveen V. Mummaneni, MD; Sanj

Introduction: Spinal cord injuries occur in approximately 17,000 people in the United States each year. The average length of hospital stay is 11 days and deep venous thrombosis (DVT) rates are reported as high as $65 \%$ in these patients. There is no consensus on the appropriate timing of chemical DVT prophylaxis for this critically injured patient cohort. The object of our study was to determine if low-molecular weight heparin was safe and effective if given within 24 hours of spinal cord injury.

Methods: TRACK SCI is a prospective observational study by the UCSF Brain and Spinal Injury Center. Protocol at this center includes administration of low-molecular weight heparin within 24 hours of spinal cord injury. Data was retrospectively reviewed to determine DVT rate, pulmonary embolism (PE) rate and hemorrhagic complications.

Results: 49 patients were enrolled in the study. There were 3 DVTs $(6.1 \%), 2$ PEs (4.1\%), and no hemorrhagic complications. Regression modeling did not find an association between DVT/ $\mathrm{PE}$ and age, American Spinal Injury Association (ASIA) grade, gender, race or having undergone a neurosurgical procedure.

Conclusion: A standardized protocol in which LMWH is 
given to SCI patients within 24 hours of injury is effective in keeping VTE at the lower end of the reported range and is safe with a zero rate of adverse bleeding events.

274 In vivo Quantitative T2 and Free Water Elimination Diffusion MRI Remote from the Lesion Site in Rat Spinal Cord Injury

Olesya Motovylyak, BS; Brian Schmit, PhD; Shekar N. Kurpad, MD, PhD; Matthew Budde, PhD

Introduction: The goal of this project was to quantify water in different tissue compartments of the cervical spinal cord following a thoracic injury using quantitative T2 imaging (qT2) and compare its sensitivity to injury with diffusion tensor imaging with free water elimination (DTIFWE). qT2 is sensitive to free water and thus has potential to measure edema. Edema is a secondary injury factor and could be used as a biomarker of injury away from epicenter.

Methods: Female Sprague-Dawley rats $(n=40)$ underwent a controlled $\mathrm{T} 8$ contusion injury at one of four severities. The rats underwent in vivo qT2 and diffusion scans at 2, 30, and 90 days post injury using a 9.4T Bruker horizontal bore scanner. A CarrPurcell-Meiboom-Gill (CPMG) spin-echo sequence was utilized for qT2 with TR/TE/echo spacing $=6000 / 6.5 / 6.5 \mathrm{~ms}$, number of echoes $=40, \mathrm{NEX}=2$, and slice thickness of $1.75 \mathrm{~mm}$. MERA Toolbox (MERA toolbox Version 2.02, Mark D Does, Vanderbilt University, TN) was used to compute $\mathrm{T} 2$ distributions from the signal decay curves. Diffusion weighted images were acquired with a 4-shot echo-planar spin-echo sequence (TE $=27 \mathrm{~ms}$; TR $>1800$ $\mathrm{ms}$ ) and custom routines were used to reconstruct and register DTIFWE maps [1]. A two-way mixed effects ANOVA was performed to identify effects of time and injury severity in both ROI and pixel-wise analyses.

Results: Axial diffusivity (ADFWE) and mean diffusion (MDFWE) in the white matter of cervical spinal cord after a thoracic injury decreased with time post injury (Figure 1). Mixedeffects ANOVA revealed a significant effect of time on ADFWE $(p=0.008)$ and MDFWE $(p=0.026)$ in the white matter ROIs, but no significance was observed for injury severity and interaction. qT2 parameters, including myelin water fraction, tissue water fraction, and free water fraction, were not significant for time, injury severity, or interaction.

Conclusion: DTIFWE is a better predictor of injury severity than qT2 chronically.

275 Decreased Segregation of Whole-Brain Resting-state Network in Spinal Cord Injury: A Graph Theoretical Analysis

Mayank Kaushal, MD, MBA; Akinwunmi Oni-Orisan, MD; Gang (Jeff) Chen; Wenjun Li, PhD; John Leschke; B. Douglas Ward, MS; Benjamin Kalinosky, PhD; Matthew Budde, PhD; Brian Schmit, PhD; Vaishnavi Muqeet, MD; Shekar N. Kurpad, $\mathrm{MD}, \mathrm{PhD}$

Introduction: There has been increasing focus on studying large-scale brain networks using graph theory to characterize resting-state functional connectivity. By describing the brain networks in terms of nodes or regions of interest (ROI) and edges or connections between the nodes, various network properties can be evaluated with the help of quantifiable metrics. The present study demonstrates changes to the property of segregation, which evaluates local connectivity of brain networks, in spinal cord injury (SCI).

Methods: After obtaining the necessary IRB approval, resting-state functional magnetic resonance imaging (rs-fMRI) was performed on 15 subjects with chronic, cervical SCI (ASIA A) and 15 controls that were intact neurologically. The rs-fMRI data was preprocessed to correct for artifacts and then divided into 264 ROIs based on functional atlas from Power et al. The average time series extracted from each ROI was correlated with every other ROI to obtain connectivity matrices. Correlation coefficient (CC), which measures the connectedness of node's neighbor with each other, and modularity (M), which calculates the extent of division of a network into non-overlapping groups were selected to network segregation. $\mathrm{CC}$ and $\mathrm{M}$ were computed at multiple incremental cost thresholds ( $\%$ of total possible connections) and compared between SCI and controls.

Results: The whole-brain network showed differences between the study groups for both metrics at multiple cost thresholds with CC and M found to be significantly decreased in SCI compared to controls

Conclusion: The presence of significant differences in the metrics studying system segregation demonstrates the utility of graph theory in evaluating information processing carried out within specialized regions. The findings illustrate that disruption to information transmission between the brain and the spinal cord leads to decreased functional specialization within the whole-brain network. This has the potential to facilitate noninvasive biomarker development for improving the monitoring of functional improvement conferred by various therapeutic approaches.

276 Efficacy and Safety of Riluzole in Acute Spinal Cord Injury (SCI). Rationale and Design of AOSpine Phase III Multi-center Double Blinded Randomized Controlled Trial. (RISCIS).

Michael G. Fehlings, MD, PhD, FRCS(C), FACS; Branko Kopjar, MD; Robert G. Grossman, MD

Introduction: There is convincing evidence from the preclinical realm that the pharmacologic agent riluzole attenuates certain aspects of the secondary injury cascade leading to diminished neurological tissue destruction in animal SCI models. The safety and pharmacokinetic profile of riluzole have been studied in a multicenter pilot study in 36 patients. Efficacy of riluzole in acute human SCI has not been established.

Methods: This ongoing multi-center, international doubleblinded phase III RCT will enroll 351 patients with acute C4 C8 SCI and ASIA Impairment Grade A, B or C randomized $1: 1$ to riluzole and placebo. Primary outcome is the change in ASIA Motor Score (AMS) between baseline and 180 days. Other outcomes include ASIA Upper and Lower Extremity MS; ASIA Sensory Score; ASIA grade; SCIM); SF-36v2; EQ-5D and GRASSP. Two-stage sequential adaptive trial statistical design has $90 \%$ power to detect 9 points difference in the ASIA Motor Score at one-sided alpha $=.025$.

Results: A matched cohort analysis performed in the Phase I study showed that riluzole treated cervical SCI patients experienced an additional 15.5 points in AMS recovery at 90 days post injury. Although the phase I study was underpowered to investigate efficacy the current phase III study is poised to definitive address this question. To date, 98 subjects have been enrolled. Average age of the enrolled subjects is 48.0 (SD 16.4); $84 \%$ males. ASIA at arrival and Pre-Injury status, ASIA Grade A (48\%), B (27\%), C (25\%). GRASSP 64.6 (SD 61.4), SF35v2 PCS 53.0 (SD 9.0) SF36v2 MCS 54.3 (SD 11.5).

Conclusion: This is a Phase III study of riluzole in acute SCI. 
277 AOSpine Subaxial Cervical Spine Injury Classification System: The relationship Between Morphology, Admission and Long-term ASIA Motor Score

Harry Melrose Mushlin, MD; Matthew J Kole; Timothy Chryssikos, MD, PhD; Gregory Cannarsa, MD; Bizhan Aarabi, MD

Introduction: The AOSpine Subaxial Cervical Spine Injury Classification System was introduced to improve communication, clinical management and research. Here, the AOSpine Subaxial Cervical Spine Injury Classification System was applied to a diverse cohort of spinal cord injury patients with correlated neurological motor scores and injury severity at admission and follow up.

Methods: A retrospective study was performed from 20132015 for patients with subaxial cervical spine injuries at $\mathrm{R}$ Adams Cowley Shock Trauma Center. Patients were classified by AOSpine Subaxial Cervical Spine Injury Classification System. Six major groups were identified for analysis: A0M3, A1/A2, A3/ A4, B2, B3, and C. Admission and follow up ASIA motor scores (AMS) and incomplete versus complete injury severity were recorded.

Results: Eighty-two patients were identified for analysis. Mean time to follow up was 11 months (6-33 months). The dominant morphology types were A0M3 in 36, A1/A2 in 4, A3/A4 in $9, \mathrm{~B} 2$ in $8, \mathrm{~B} 3$ in 11 , and $\mathrm{C}$ in 14 patients. There was significant improvement in ASIA motor score at admission versus follow up for three classes: A0M3, B3, C. Classes A0M3 had a significantly higher AMS (70) compared to B3 and $\mathrm{C}$ (41,38 respectively) at admission ( $\mathrm{p}=0.01 ; \mathrm{p}=0.02)$. Class $\mathrm{A} 0 \mathrm{M} 3$ and $\mathrm{B} 2$ had significantly higher AMS (90,95 respectively) at follow up compared to $\mathrm{C}$ and $\mathrm{A} 3 / \mathrm{A} 4$ (56,48 respectively) ( $\mathrm{p}=0.02 ; \mathrm{p}=0.01 ; \mathrm{p}=0.02$; $\mathrm{p}=0.03$ ). There were significantly more complete patients at admission in class $\mathrm{C}$ and $\mathrm{A} 3 \& 4$ compared to classes $\mathrm{A} 0 \mathrm{M} 3$ and $\mathrm{B} 3$ $(\mathrm{p}=0.005 ; \mathrm{p}=0.05 ; \mathrm{p}=<0.0001 ; \mathrm{p}=0.01)$. Class $\mathrm{C}$ had significantly more incomplete patients at follow up versus admission $(\mathrm{p}=0.04)$.

Conclusion: This is a comprehensive cohort which studied admission and follow-up AMS/injury severity of subtypes of the AOSpine Subaxial Cervical Spine Injury Classification System. We successfully classified injuries and showed the use of predicting initial AMS and follow up injury severity and motor scores across classes.

278 A Preclinical Study of Advanced Diffusion MRI as a Prognostic Marker of Acute Spinal Cord Trauma

Nathan Skinner, PhD; Antje Kroner-Milsch, MD, PhD; Brian Schmit, PhD; Shekar N. Kurpad, MD, PhD; Matthew Budde, PhD

Introduction: Early medical care after spinal cord injury (SCI) is paramount to recovery. Current clinical methods of SCI assessment have significant gaps in predicting functional outcome, and while magnetic resonance imaging (MRI) has aided diagnosis, it has only moderate prognostic power. Diffusion tensor imaging (DTI) has shown promise as a predictive biomarker, but its clinical adoption has been limited. The goal of this work was to assess a novel diffusion MRI technique, double diffusion encoding (DDE), in its ability to predict outcome in a rat model of SCI.

Methods: Traumatic spinal cord contusion injury was induced in $\operatorname{rats}(\mathrm{n}=52)$, which underwent MRI at 2 and 30 days postinjury on a 9.4T MRI. The DDE technique, which was developed specifically to detect acute axonal injury, was performed at the lesion epicenter and compared to DTI. Weekly functional scoring (Basso, Beattie, and Breshnahan;BBB) and terminal histology was performed. Linear regression was used to assess the relationship between MRI metrics either BBB scores or quantitative histological measures.

Results: DDE at 2 days post injury was strongly associated with functional outcomes at 30 days post injury, while DTI at the same timepoint was not significantly related to outcome. DDE improved sensitivity to axonal injury by suppressing the signals from edema, extracellular fluid, and cerebrospinal fluid and other pathologies that can confound DTI. A whole-cord quantitative measure of injury was obtained in a short acquisition time ( $<3$ mins), with the spatial pattern of injury to the cord clearly observed in the individual animals.

Conclusion: We have demonstrated a novel diffusion MRIbased technique to reliably assess the severity of spinal cord traumatic injury in the acute setting that was prognostic of functional outcome. Future studies in acute SCI patients are needed to realize the clinical applications emerging from these promising preclinical findings.

279 Safety of Autologous Human Schwann Cell Transplantation in Chronic Spinal Cord Injury (SCI)

Stephen Shelby Burks, BA, MD; Kim D Anderson, PhD; James D. Guest, MD, PhD; Dalton Dietrich, PhD; Barth A. Green, MD, FACS; Allan D. Levi, MD, PhD

Introduction: Schwann cells (SCs) are excellent candidates for transplantation into humans with SCI. SCs, glial cells essential for repair in damaged nerves, have shown the ability to survive and provide repair when employed in experimental paradigms of SCI in rodents. Large numbers of autologous human SCs can be obtained for implantation after a peripheral nerve biopsy. Autologous cells offer important safety advantages that include minimal risk of disease transfer, low risk of tumorigenicity, and the absence of immunosuppressive medication requirements.

Methods: A Phase I clinical trial was conducted to evaluate the safety of autologous human SC transplantation into the injury epicenter of 10 participants with chronic SCI. The trial was an open-label, unblinded, non-randomized, non-placebo controlled study with a cavity-filling dose design and a prescribed exercise conditioning and locomotor rehabilitation program. Participants injury levels were C5-T12 with neurologically complete or incomplete, trauma-induced spinal lesions. Autologous SCs were cultured in vitro from a sural nerve harvested from each participant and injected into the epicenter of the spinal lesion. Outcome measures for safety were adverse events, stability of neurologic level, absence of detectable mass lesion, and lack of clinically significant worsening of neuropathic pain.

Results: Six months post-transplantation, there were no surgical, medical, or neurological complications to indicate that the dose or procedure for the cell transplantation were unsafe. There were no adverse events or serious adverse events related to the cell therapy as well as no evidence of additional spinal cord damage, mass lesion, or syrinx formation. There was no worsening of pre-existing neuropathic pain. Some participants gained clinically detectable motor or sensory improvements.

Conclusion: Preliminary evidence indicate safety for intraspinal transplantation in chronic SCI.

280 Cost-utility Analysis of Cervical Deformity Surgeries Using One-year Outcomes.

Peter G Passias, MD; Gregory W, Poorman; Rabia Qureshi, MD; Hamid Hassanzadeh, MD; Samantha R. Horn; Amit Jain BS; Michael P Kelly, MD; Richard A. Hostin, MD; Frank Segreto; Cole Bortz, BA; Muhammad Burhan Ud Din Janjua; Christopher P. Ames, MD; Justin S. Smith 
Introduction: Cost-utility analysis, a special case of costeffectiveness analysis, estimates the ratio between the cost of an intervention to the benefit it produces in number of qualityadjusted life years. Cervical deformity correction has not been evaluated in terms of cost-utility and in the context of value-based health care.

Methods: Retrospective review of a prospective cervical deformity database. Cervical deformity patients with 1-year follow-up after surgical correction were included. Cervical deformity was defined as: kyphosis $\left(\mathrm{C} 2-7\right.$ Cobb-angle $\left.>10^{\circ}\right)$, cervicalscoliosis (coronal-Cobb-angle $>10^{\circ}$ ), positive-cervical-sagittalmalalignment (C2-C7 sagittal-vertical-axis $>4 \mathrm{~cm}$ or T1-C6 $>10$ o), or horizontal-gaze-impairment (chin-brow-vertical-angle $>250$ ). Quality-adjusted-life-years were calculated by both EuroQol-5D (EQ5D) quality-of-life and NDI mapped to SF6D index. Costs were assigned using Medicare 1-year average reimbursement for: 9+ level posterior fusions (PF), 4-8 level PF, 4-8 level PF with anterior fusion (AF), 2-3 level PF with AF, 4-8 level AF, and 4-8 level posterior refusion. Reoperations and deaths were added to cost and subtracted from utility respectively. QALY per dollar spent was calculated using standardized methodology at 1-year and subsequent time-points relying on maintenance of 1-year utility.

Results: 84 postoperative patients were isolated (age: 61.2 years, $60 \%$ female, BMI: 30.1 , average-levels-fused: 7.2 , osteotomy used: $50 \%)$. Costs associated with index procedures were: 9+ level PF $(\$ 76,617)$, 4-8 level PF (\$40,596), 4-8 level PF with AF $(\$ 67,098), 4-8$ level AF $(\$ 31,392)$ and 4-8 level posterior refusion $(\$ 35,371)$. Average 1-year reimbursement of surgery was $\$ 55,097$ at 1 -year with 8 revisions and 3 deaths accounted for. Cost per QALY gained to 1-year follow-up was $\$ 646,958$ by eq5d and $\$ 477,316$ by NDI SF6D. If 1 -year benefit is sustained, upper threshold of cost-effectiveness is reached 3-4.5 years after intervention.

Conclusion: Medicare 1-year average reimbursement compared to 1 -year quality adjusted life year described $\$ 646,958$ by eq5d and $\$ 477,316$ by NDI SF6D. Cervical deformity surgeries reach cost-effectiveness thresholds when benefit is sustained 3-4.5 years.

281 Knowledge and Attitude of Spine Surgeons Regarding Radiation Exposure

Asdrubal Falavigna, PhD; Miguel Bertelli Ramos; Alexandre Sadao Iutaka; Cristiano M Menezes, MD; Nestor Taboada

Introduction: Questionnaires evaluating spine surgeons' knowledge and attitude regarding radiation exposure are scarce in literature. Spine surgeons are exposed to high amounts of radiation from fluoroscopy procedures throughout their lifetime (1, 2 ). Good practices may minimize this exposure, requiring prior knowledge of radiation basics and their application in a spine surgery scenario.

Methods: A questionnaire for spine surgeons was elaborated comprising questions on general information and surgeons' perceptions and attitudes regarding radiation exposure during spine surgery. Questions were sent via Survey Monkey tool to spine surgeons who were members of AOSpine Latin America, during the period between December 15th 2016 and January 15th 2017. The answers from countries that had more than 40 surgeons were compared.

Results: The questionnaire was answered by 371 surgeons. There were more orthopaedic surgeons $(n=212)$ than neurosurgeons $(n=159)$. Most surgeons $(54.2 \%)$ were very experienced. Thyroid protector was not used by 133 surgeons (35.8\%). Lead glasses were used by $75(20.2 \%)$ and lead gloves by $26(7 \%)$. The dosimeter badge was never or rarely used by $281(75.7 \%)$ and 321 $(86.5 \%)$ don't know their own dose limits. Only 124 surgeons $(33.4 \%)$ answered that staying on the image intensifier side during lateral lumbar fluoroscopy reduces the surgeon's exposure. Orthopaedic surgeons answered the best position of the surgeon during lateral lumbar fluoroscopy correctly more often than neurosurgeons $(\mathrm{p}=0.01)$. Usage of thyroid shield, staying behind the $\mathrm{X}$-ray source and usage of navigation were greater among neurosurgeons than among orthopaedic surgeons, $\mathrm{p}=0.005, \mathrm{p}=0.006$ and $\mathrm{p}<0.001$, respectively. Staying behind the $\mathrm{X}$-ray source and usage of navigation were also greater among more experienced surgeons than among less experienced ones, respectively, $\mathrm{p}=0.01$ and $\mathrm{p}=0.005$.Surgeons from Brazil and Colombia reported greater use of the thyroid protector than surgeons from Mexico and Argentina $(\mathrm{p}<0.001)$. Stepping back during fluoroscopy was also significantly greater in the former than in the latter $(\mathrm{p}=0.01)$. However, pulsed-mode fluoroscopy is significantly more used in Mexico and Argentina when compared to Brazil and Colombia $(\mathrm{p}<0.0001)$.

Conclusion: Spine surgeons do not know sufficient or take a proactive attitude towards radiation exposure.

282 Unified Workforce Analysis of Spine Faculty in Neurological and Orthopedic Surgery Residency Programs

\section{Alexander F. Post, MD; Tanvir Choudhri, MD}

Introduction: We present a unified workforce analysis of academic, spine-focused faculty at U.S. neurological and orthopedic surgery residency programs.

Methods: 278 ACGME-training programs (110 neurosurgical, 168 orthopedic) were queried to identify 945 full-time spinal surgery faculty (designated by fellowship training or case volume $>75 \%$ spine). Academic rank, gender, board certification and extent of training were assessed.

Results: Specialty breakdown showed more orthopedic surgeons [NS:OS $=435(46 \%): 510(54 \%), \mathrm{p}<0.016]$ with a similar dispersion of academic rank: Instructors [NS:OS $=4(0.4 \%$ of all surgeons) : $14(1.5 \%$ of all surgeons $), p=0.039$ ], Assistant Professors [NS:OS = 150(15.9\%) : 169(17.9\%), $\mathrm{p}=0.31, \mathrm{~ns}$, Associate Professors [NS:OS $=91(9.6 \%): 79(8.4 \%), \mathrm{p}=0.40$, ns], Professors [NS:OS $=101(10.7 \%): 112(11.9 \%), \mathrm{p}=0.49$, ns], no academic rank listed [NS:OS $=89(9.4 \%): 139(14.4 \%)$ $\mathrm{p}=0.0011]$. Gender breakdown showed male predominance in neurosurgery [M:F $=403(92.6 \%): 32(7.4 \%)$, binomial test $\mathrm{p}<$ $0,0001]$, orthopedic surgery $[\mathrm{M}: \mathrm{F}=493(96.7 \%): 17(3.3 \%), \mathrm{p}<$ $0.0001]$ and overall $[\mathrm{M}: \mathrm{F}=896(94.8 \%): 49(5.2 \%), \mathrm{p}<0.0001]$ as well as within each academic rank: Instructors $[\mathrm{M}: \mathrm{F}=15(1.6 \%)$ : $3(0.3 \%), \mathrm{p}<0.0075]$, Assistant Professors [M:F = $294(31.1 \%)$ : $25(2.6 \%), \mathrm{p}<0.0001]$, Associate Professors [M:F $=160(16.9 \%)$ $: 10(1.1 \%), \mathrm{p}<0.0001]$, Professors $[\mathrm{M}: \mathrm{F}=209(22.1 \%): 4(0.4 \%)$ $\mathrm{p}<0.0001]$, no rank listed [M:F $=220(23.3 \%): 5(0.5 \%), \mathrm{p}<$ $0.0001]$. Verified completion of primary, secondary and tertiary residencies and primary, secondary and tertiary fellowships did not show substantial difference between groups. Board certification was similar between the groups with a higher rate of board eligible status in the neurosurgical group.

Conclusion: We present a unified picture of academic spine teaching faculty. Results may be used for benchmark purposes, residency program self-assessment or to aid those entering residency as to where to pursue spine training. 
283 Beyond Biology: Impact of Marital Status and SocioEconomic-index on Outcomes of Spinal Cord Tumors, Analysis from A National Cancer Registry.

Mohammed Ali Alvi, MD; Waseem Wahood, MS; Panagiotis Kerezoudis; F.M Moinuddin, MBBS, Ph.D; Sandy Goncalves; Yagiz Ugur Yolcu; Mohamed Elminawy, MBBCh; Anshit Goyal, MBBS; Redab Alkhataybeh; Mohamad Bydon, MD

Introduction: The impact of marital-status and living arrangement on health through modified health behaviours and social networks has been well-established in the literature with many experts claiming a "protective" effect that married life confers to individuals. Herein, we sought to study the impact of marital and socio-economic-status(SES) on outcomes of spinal-cord tumors.

Methods: Surveillance, Epidemiology and End Results (SEER) program was utilized for abstracting patients with spinal cord tumors between 2004 and 2014. Patients were grouped into 4 groups based on their marital status: single/never married, married/living together, divorced/separated and widowed. Socioeconomic status was calculated using a validated methodology. Kaplan-meier curves and multivariable logistic and cox regression were used to investigate the relationship between marital and socio-economic status and mortality rate of patients with available follow-up information.

Results: A total of 1188 patients with spinal tumors were identified, of which $683(57.5 \%)$ were female and 505(42.5\%) were male. Patients were categorized into four groups: $241(20.3 \%)$ single patients, $732(61.6 \%)$ married patients/patients with domestic partner, 109(9.2\%) divorced/separated patients, and $106(8.9 \%)$ widowed patients. Compared to married patients, divorced/separated and widowed patients had a higher mortality rate (HR:1.76, 95\% CI: 1.1 to $2.9, \mathrm{p}=0.02$ and HR:2.01, $95 \% \mathrm{CI}: 1.3$ to $3.1, \mathrm{p}=$ 0.001 respectively). Male patients, compared to female patients, were associated with lower survival (HR: $1.42,95 \%$ CI: 1.03 to $1.9, \mathrm{p}=0.03$ ). Lastly, patients belonging to higher SES were found to have a lower mortality rate (HR: 0.77 , CI $0.55-1.08, \mathrm{p}=0.143)$.

Conclusion: Consistent with other studies, unmarried and widowed patients, patients belonging to lower SES, and male patients were found to have a higher risk of mortality. Thus, these factors should be accounted for while tailoring the treatment plan for such patients.

284 Social Factors Associated with Surgical Utilization by Patients Evaluated for Degenerative Spine Pathologies

Zachary Pennington; Daniel Lubelski, MD; Joseph E Tanenbaum, BA; A. Karim Ahmed; Marissa Rosato; Daniel M. Sciubba, MD

Introduction: Elective spine care generates 50-132 million outpatient visits annually. However, $55-82 \%$ of specialty referrals may be inappropriate, raising the overall cost of spine care. We seek to determine which pre-visit factors are most strongly associated with surgical utilization by patients seeking surgical evaluation for degenerative spine pathologies.

Methods: All new patients with complete new patient paperwork seen by the senior author between May 2016 and April 2017 for degenerative spine pathologies were reviewed. We collected data on review of systems, concurrent medical issues, demographics, and previous spine care. Multivariable logistic regressions were performed to determine which factors were associated with surgical candidacy and surgical utilization.

Results: Our cohort included 90 non-surgical candidates (55.6\% female; mean age 58.4y.o.), 27 surgical candidates who forwent surgery (55.6\% female; 57.9y.o.), and 27 patients who underwent surgery (48.1\% female; 60.9y.o.). In multivariable analysis, factors associated with surgical utilization were BMI (95\% CI: [1.0-1.2]; $\mathrm{p}=0.02)$, male sex ([1.1-4.3]; $\mathrm{p}=0.03)$, numbness ([1.0-3.3]; $\mathrm{p}=0.05)$, number of allergies ([1.0-2.0]; $\mathrm{p}$ $=0.03)$, being employed $([1.2-7.2] ; \mathrm{p}=0.02)$ or retired $([1.5-9.7]$; $\mathrm{p}=0.005) \mathrm{v}$. unemployed, number of complaints on the review of systems $([0.8-1.0] ; \mathrm{p}=0.01)$, non-spinal pain $([1.4-4.7] ; \mathrm{p}=$ $0.003)$, and report of pain as chronic ([1.1-4.0]; $\mathrm{p}=0.02)$.

Conclusion: Our results suggest that many of the factors associated with surgical utilization are non-spinal. Given that the success of a care plan is dependent upon the willingness of a patient to adhere to it, our results suggest that patients being considered for spine specialist referral may benefit from their physicians considering non-clinical as well as clinical characteristics.

285 Alcohol-use on Complication and Readmission Rates After Elective Spinal Fusion (e"2 Levels) for Adult Spine Deformity

Aladine A. Elsamadicy, BE; Owoicho Adogwa, M.D., M.P.H; Victoria D Vuong; Amanda Sergesketter; Joseph S. Cheng, MD, MS; Carlos Antonio Bagley, MD; Isaac O. Karikari, MD

Introduction: Alcohol use has been shown to affect surgical outcomes. However, it is unknown what effect alcohol use has on postoperative complications or readmission rates in spinal fusion surgery. The aim of this study is to determine the impact of preoperative alcohol use on 30-day readmission rates or the complications profile after adult elective spinal fusion for deformity correction (=2 levels).

Methods: The medical records of 1010 adult patients undergoing elective spinal fusion (=2 levels) for spinal deformities at a major academic institution from 2005 to 2015 were reviewed. We identified $317(31.4 \%)$ patients who used alcohol pre-operatively and $693(68.6 \%)$ patients who had no alcohol use preoperatively. Patient demographics, comorbidities, intra- and 30-day post-operative complication and readmission rates were collected for each patient. The primary outcome investigated in this study was the rate of 30-day readmissions and postoperative complication rates.

Results: Baseline characteristics were similar between both cohorts, Table 1 . Intraoperative variables and the immediate postoperative complications profile were mostly similar between both cohorts, with the exception of estimated blood loss, number of transfusions, length of stay, and admissions to the intensive care unit, Tables 2 and 3. Overall, there was no significant difference between the 30 -day readmission rates or complications profile between the two cohorts, Table 4 .

Conclusion: Our study suggests there is no significant difference in 30-day readmission or complication rates among adult patients with or without preoperative alcohol use undergoing elective correction of spinal deformities.

286 Cost and Effectiveness of Allogenic Bone Substrate for Spinal Arthrodesis

Stephen Lewis Reintjes, MD; Elliot Pressman, BS; Alex Niefert, BS; Quan Tran, BS, MS; Fernando L. Vale, MD

Introduction: Bone graft substitutes are widely used in spine surgery although autologous bone graft remains the gold standard in spinal arthrodesis. Recently there has been a rise in allograft as a bone substitute or autograft extender for long constructs. However, the effectiveness of various bone graft substitutes on fusion rates and cost of these products has never been studied. Several new cellular allograft materials were introduced evaluated the allograft 
products used at our institution and their affects on fusion rates.

Methods: We retrospectively reviewed 934 patients who underwent a fusion surgery at our tertiary care referral center between 2011-2013. After excluding 366 patients for not meeting eligibility criteria, we included 288 patients with cervical fusions, 10 patients thoracic fusions and 270 patients with lumbar fusions. We divided patients between levels of surgery and bone graft material and performed an analysis to see which factors affected fusion rates. We also performed a cost analysis of each allograft material to determine a cost per level equivalent for each substrate.

Results: Successful fusion rates for cervical, thoracic, and lumbar patients were $93.7 \%, 100 \%$, and $89.6 \%$ respectively. At our institution we use several products for fusion. Mainly Osteocel $(44 \%)$, Trinity $(20 \%)$, DBX $(12 \%)$, Grafton $(12 \%)$, BMP (8\%), Cancellous Chips (5\%), Connexus (4\%). There were high fusion rates among all allograft products: Osteocel $92 \%$ $(\mathrm{p}=0.996)$, Trinity $91 \%(\mathrm{p}=0.995), \mathrm{DBX} 89 \%(\mathrm{p}=0.995)$, Grafton $88 \%$ ( $\mathrm{p}=0.995)$, BMP 100\% ( $\mathrm{p}=0.995)$, Cancellous Chips $100 \%$ $(\mathrm{p}=0.996)$, Connexus $89 \%(\mathrm{p}=0.995)$, mixed $96 \%(0.995)$.

Conclusion: There are many factors involved in successful arthrodesis, including choice of allograft material. Proper patient selection and meticulous technique are required for successful arthrodesis. We find that fusion rates remain high over time and that new cellular allografts might not justify the cost.

287 Ranking Spine Surgeons And Practices by PatientReported Outcomes

Ahilan Sivaganesan, MD; Anthony L. Asher, MD, FACS; Mohamad Bydon, MD; Inamullah Khan, MD; Panagiotis Kerezoudis; Hui Nian, PhD; Frank Harrell; Clinton J. Devin, MD

Introduction: There is currently no means of determining which spine surgeons or centers provide the best patient-reported outcomes for a particular procedure. The primary aim of this study is to present a methodology for PRO-based, risk-adjusted rankings of spine surgeons and sites that perform elective lumbar surgery, using the Quality and Outcomes Database (QOD). The second aim is to determine whether the choice of surgeon, or QOD site, explains more variation in PROs.

Methods: All patients in the QOD who underwent elective lumbar surgery at the top 21 contributing sites were studied. Random effects regression models (Phase 1) were fit for the following outcomes, with QOD site as a fixed effect but surgeon ID as a random effect: ODI, EQ-5D, back pain and leg pain, and satisfaction. Hierarchical Bayesian models (Phase 2) were also fit for each outcome, with QOD site as a random effect and surgeon ID as a nested random effect.

Results: Our study cohort consists of 8834 patients who underwent surgery by 124 QOD surgeons, for the following diagnoses: disc herniation, recurrent disc herniation, spondylolisthesis, stenosis, adjacent segment disease, or symptomatic mechanical instability. Table 4 is based on Phase 2 non-overlapping Bayesian credible intervals demonstrate that the variance attributed to QOD site was significantly greater than the nested variance attributed to surgeon ID for ODI (0.519 vs. 0.236), EQ5D (0.444 vs. 0.171), VAS back pain $(0.367$ vs 0.158$)$, and satisfaction $(0.373$ vs, $0.140)$. Figure 1 is based on Phase 1 considerable variation is seen across QOD sites for each outcome, but variation among surgeon at any given site is also seen.

Conclusion: This study presents a novel methodology for the risk-adjusted, procedure-specific, PRO-based ranking of spine surgeons and practices. The choice of surgeon matters when considering PROs after elective lumbar surgery, however the choice of QOD site matters more.
288 Timeline of Quality of Life Improvements Postdecompression for Cervical Spondylitic Myelopathy at a Single Tertiary Center

Joey Kevin Grochmal, MD, PhD; Ahmed J Awad, MD; Matthew Budde, PhD; Shekar N. Kurpad, MD, PhD; Marjorie C. Wang, MD, MPH

Introduction: Cervical spondylitic myelopathy (CSM) is considered the most common cause of myelopathy in adults over 55 years, associated with disability and impaired quality of life $(1,2)$. It has become increasingly clear that patients may improve in quality of life measures post decompression(3), although further study is warranted to characterize the specifics of these improvements.

Methods: We did a retrospective analysis from a prospectively collected database of patients who underwent decompression for CSM at the Medical College of Wisconsin. 76 patients (mean age 55.1 years old) completed pre-operative mJOA and SF-36 self-assessment questionnaires, with follow up at 3,6, 12 and 24 months post operatively. mJOA was analyzed using one-way analysis of variance (ANOVA) and SF-36 was analyzed using the twoway ANOVA across follow-up time points and SF-36 domains.

Results: 58 patients completed 24-month follow up. mJOA significantly improved from baseline (13.14 +/- SD 2.29) and peaked at 3 months $(14.58+/-$ SD $1.73, \mathrm{p}<0.007)$, and thereafter reached a plateau $(14.23,14.23,14.39$ at 6,12 , and 24 months respectively) (fig 1). SF-36 quality of life assessment demonstrated significant improvement in multiple domains after three and six months post-op (physical function, role physical, bodily pain, vitality, social function, role emotional, and physical component scores, fig 2.), though failure to improve in others (general health, mental health, and mental component scores, fig.3).

Conclusion: Our data suggest that most improvement in both mJOA and patient reported quality of life scores occur by three months post-operatively, while certain QOL domains remain relatively unresponsive to surgical intervention. These data support current evidence that patients do experience benefit from CSM decompression, while encouraging further study into barriers associated with lack of improvement in general and mental health QOL scores.

289 Minimum Clinically Important Difference in SF-36 Scores for Use in Degenerative Cervical Myelopathy

Jetan H. Badhiwala, MD; Christopher D. Witiw, MD; Farshad Nassiri, MD; Jefferson R. Wilson, MD, PhD; Michael G. Fehlings, $\mathrm{MD}, \mathrm{PhD}, \mathrm{FRCS}(\mathrm{C}), \mathrm{FACS}$

Introduction: There has been a shift toward focus on patient reported outcomes (PROs) in spine surgery. However, the numerical scores of PROs lack immediate clinical meaning. The minimum clinically important difference (MCID) adds a dimension of interpretability to PRO scales, by defining the smallest change a patient would consider meaningful. To that end, through a posthoc analysis of 606 patients enrolled in the AOSpine CSM-NA(1) or CSM-I(2) prospective, multi-center cohort studies, we sought to determine the MCID in SF-36 Physical Component Summary (PCS) and Mental Component Summary (MSC) scores in patients undergoing surgery for degenerative cervical myelopathy (DCM).

Methods: The MCID of the SF-36 PCS and MCS were determined by distribution- and anchor-based methods comparing pre-operative to 12-month scores. Distribution-based approaches included calculation of the half standard deviation and standard error of measurement (SEM). Change in Neck Disability Index (NDI) served as the anchor: "worse" (?NDI>7.5); "unchanged" (7.5=?NDI>-7.5); "slightly improved" $(-7.5=$ ?NDI $>-15)$; and 
"markedly improved" (?NDI=-15). Receiver operating characteristic (ROC) analysis was performed to determine the change score for the MCID with even sensitivity and specificity to distinguish patients who were "slightly improved" versus "unchanged" on the NDI.

Results: The MCID for the SF-36 PCS and MCS were 4.6 and 6.8 by half standard deviation and 2.9 and 4.3 by SEM, respectively. By ROC analysis, the MCID was 3.9 for the SF-36 PCS score (Fig. 1) and 3.2 for the SF-36 MCS score (Fig 2). Using a cutoff of 4 points, the SF-36 PCS had a sensitivity of $72.2 \%$ and specificity of $68.1 \%$, and MCS $61.9 \%$ and $64.6 \%$, respectively, in separating patients who were "markedly improved" or "slightly improved" from those who were "unchanged" or "worse".

Conclusion: We found the MCID of the SF-36 PCS and MCS to be 4 points. This will facilitate use of the SF-36 in future studies of DCM.

290 The Epidemiology of Spinal Schwannoma in the United States Between 2006-2014.

Ghaith Habboub; Min Lang; Shahed Tish; Quinn T. Ostrom; Samantha Colby; Jaes C Jones, BS; Josie Volovetz; Alex M Witek, MD; Louis Ross; Pablo F. Recinos, MD; Richard P. Schlenk, MD; Carol Kruchko; Varun Kshettry, MD; Jill Barnholtz-Sloan

Introduction: Spinal meningioma is the most common intradural extramedullary spinal tumor followed by spinal schwannoma.1,3 The previous literature on the epidemiology of spinal schwannoma were generally hospital based rather than population based 2,4,5 The Benign Brain Tumor Cancer Registries Amendment Act mandated registration of all benign central nervous system tumors in the United States starting from 2004, thus allowing for a US population-based resource for investigation. This article describes the epidemiology of spinal schwannoma and compares its distribution to spinal meningioma distribution.

Methods: The Central Brain Tumor Registry of the United States(CBTRUS) data were used for analysis.6 CBTRUS combines data from the Center for Disease Control's National Program of Cancer Registries and the NCI's Surveillance Epidemiology and End Results Progam which represents $\sim 99 \%$ of the US population. The following criteria were used: diagnosis years 2006 to 2014, non-malignant spinal schwannoma and meningioma as defined by ICD-0-3 histology codes(9560/0 for spinal schwannoma, and 9530/0, 9531/0, 9532/0, 9533/0, 9534/0, 9537/0 for spinal meningioma) and ICD-0-3 site codes used included C70.1(spinal meninges), C72.0(spinal cord), C72.1(cauda equina).

Results: There were 6179 spinal schwannoma cases and 8254 spinal meningioma cases between the years 2006-2014. The peak adjusted incidence rates were in the 65-74 years range in spinal schwannoma and 75-84 in spinal meningioma. The rate ratio of male to female was 1.16 in spinal schwannoma and 0.28 for spinal meningioma. The rate distribution of spinal schwannoma across age groups and gender is shown in the attached figures.

Conclusion: The overall incidence rate of spinal schwannoma is 0.21 per 100,000 . Spinal schwannoma is slightly more common in males $($ ratio $=1.16)$ while spinal meningioma has significantly higher rate in females(ratio= 3.6). We noted an increase in the incidence rate of spinal schwannoma across time which could be due to better documentation, increase in imaging/operations, or a real increase in the tumor rate.

291 Morphometrics Predicts Overall Survival in Patients with Lung, Breast, Prostate, or Myeloma Spine Metastases, Independent of Cancer Histology or Spine Metastatic Burden

Hesham Mostafa Zakaria, MD; Lara Walsh Massie, MD;
Azam Basheer, MD; Lonni Schultz, PhD; Ian Yu Lee, MD; Brent Griffith, MD; Farzan Siddiqui; Victor W. Chang, MD

Introduction: Predicting survival of patients with spinal metastases would help stratify treatments between aggressive to palliation.1, 2 Morphometrics has been used to predict postoperative morbidity and mortality. 3 This study evaluates whether morphometrics is predictive of survival in patients with lung, breast, prostate, or multiple myeloma spinal metastasis.

Methods: We identified patients with lung, breast, prostate, or myeloma spine metastases from a stereotactic body radiation therapy (SBRT) registry. Morphometric measurements were taken of the psoas from the most recent CT scan, and sizes were split into tertiles (thirds). Overall survival and hazard ratios were calculated with multivariate cox proportional hazards regression analyses.

Results: In 417 patients with spinal metastases, $40 \%$ had lung cancer, $27 \%$ breast, $21 \%$ prostate, and $11 \%$ myeloma. The average age was 66.3 , with $50 \%$ male and $52 \%$ Caucasian. The median overall survival was $173 \mathrm{~d}(95 \% \mathrm{CI}=140204 \mathrm{~d})$ and was not associated with age, sex, race, number of levels treated, or total SBRT target volume. Myeloma patients had a longer survival $(\mathrm{p}<0.001)$, but no other tumor specific survival was detected. Multivariate analysis showed patients in the lowest psoas tertile had shorter survival $(115 \mathrm{~d}, 95 \% \mathrm{CI}=91153 \mathrm{~d})$ as compared to the middle $(154 \mathrm{~d}$, $95 \% \mathrm{CI}=109218, \mathrm{p}=0.013)$ and largest tertile $(299 \mathrm{~d}, 95 \% \mathrm{CI}=228$ $435, \mathrm{p}<0.001)$. The middle tertile had shorter survival than the largest tertile, $p=0.015$. Patients with psoas sizes above the median had longer survival $(253 \mathrm{~d}, 95 \% \mathrm{CI}=204319 \mathrm{~d})$ than those below the median $(124 d, 95 \% \mathrm{CI}=99$ 157d), $\mathrm{p}<0.001$. Kaplan-Meier survival curves visually represent differences in survival.

Conclusion: In patients with spine metastases, morphometric analysis of psoas muscle size can be used to identify patients who are at risk for shorter survival, regardless of tumor histology or spine tumor burden (total SBRT volume). This information can be used to help with surgical decision making in patients with the same burden of disease, as patients with a small psoas sizes are at higher risk of death.

292 Predictive Factors Leading to Readmission Within 30 Days in Patients Undergoing Surgery for Spinal Metastases: A Multivariate Logistic Regression Analysis

Jay I. Kumar, BS; Vijay Yanamadala, MD, MBA; Ganesh Shankar, MD, PhD; Bryan D. Choi, MD; John H. Shin, MD

Introduction: Readmission within 30 days after complex spine surgery is considered a "never event" by Medicare but remains a reality in $30-35 \%$ of cases after surgical treatment of spinal metastases. We present a single-center experience of readmissions in 185 consecutive patients who underwent surgery for spinal metastases and assess predictive factors for readmission.

Methods: Charts of 185 patients who underwent surgery for spinal metastases over five years from October 2011 through February 2017 were reviewed for unplanned readmission within 30 days and possible contributing factors. Multivariate logistic regression was performed for patient demographic and surgical parameters that predict readmission.

Results: The rate of unplanned readmission within 30 days was $28 \%$. The most common reason for readmission was pain, accounting for $25 \%$ of all readmissions. Medical factors accounted for $54 \%$ of readmissions: failure to thrive, $14 \%$; fever, $12 \%$; altered mental status, $12 \%$; pulmonary embolism, $4 \%$; and miscellaneous, $12 \%$. Surgical factors accounted for $22 \%$ of readmissions: wound infection, 14\%; new neurologic deficit, 6\%; and miscellaneous, $2 \%$. The number of levels with metastatic disease, whether a patient had $>3$ levels with metastatic disease, and whether a 
patient received chemo within 30 days before surgery were all predictive of readmission at a statistically significant threshold of $\mathrm{p}<0.05$. Female sex, receiving chemo at any point before surgery, and receiving radiation to the spine at any point before surgery tended to predict readmission but were not statistically significant $(\mathrm{p}<0.11)$.

Conclusion: Surgery for spinal metastases is associated with a significant readmission rate. Pain and medical factors accounted for most of readmissions. Advanced nature of disease and recent history of chemotherapy are predictive of readmission and should be taken into account when deciding on discharge disposition for patients after surgery for spinal metastases. Enhanced coordination of post-operative care between surgical and medical teams may help reduce readmission rates.

293 Evidence from the Epidemiology, Process and Outcomes of Spine Oncology (EPOSO) Cohort: Surgical Versus Radiation Therapy for the Treatment of Cervical Metastases.

Michael Bond, MHSc, MD; Anne Versteeg; Arjun Sahgal; Peter Varga; Daniel M. Sciubba, MD; Michael Weber, MD, PhD, FRCSC, 1; Michelle J. Clarke, MD; Laurence D. Rhines, MD; Stefano Boriani, MD; Chetan Bettegowda, MD, PhD; Michael G. Fehlings, MD, PhD, FRCS(C), FACS

Introduction: Cervical metastases have unique clinical considerations because of complex neighbouring anatomy, and the unique biomechanical regions within the cervical spine. The literature regarding cervical metastases is limited to retrospective studies without comparison of different treatment regimens. The purpose of this study was therefore to compare surgery (+/- radiation) with radiation alone for the management of cervical metastases in a prospective manner.

Methods: Patients treated with surgery and/or radiotherapy for cervical metastases between August 2013 and February 2017 were identified from the Epidemiology, Process and Outcomes of Spine Oncology (EPOSO) observational cohort. Demographic, diagnostic, treatment and health related quality of life (HRQOL) (NRS Pain, EQ-5D, SF-36v2, and SOSGOQ) measures were prospectively collected at baseline, 6 weeks, 3 months, and 6 months post-intervention.

Results: Fifty-five patients treated for cervical metastases were identified: 38 underwent surgery (+/- radiation) and 17 received radiation alone. Surgically treated patients had higher SINS scores compared to the radiation-alone group (13.0 [SD 2.8 ] vs. 8.0 [SD 2.8], p<0.001) and were more likely to have mechanical neck pain $(89.5 \%$ vs. $37.5 \%, \mathrm{p}<0.001)$. Surgically treated patients presented with significantly higher NRS pain scores and lower HRQOL scores compared to the radiation alone group $(\mathrm{p}<0.05)$. From baseline to 6 months post-treatment, surgically treated patients demonstrated significant improvements in NRS pain, EQ-5D and SOSGOQ2.0 compared to non-significant improvements in the radiotherapy only group.

Conclusion: Surgically treated patients presented with significantly worse baseline pain and HRQOL scores compared to patients who underwent radiotherapy only. Preservation of pain and HRQOL was observed for patients treated with radiotherapy only compared to significant improvements for surgically treated patients.

294 Predictive Factors for Survival in a Surgical Series of Metastatic Epidural Spinal Cord Compression: A Prospective North American Multi-Centre Study in 142 patients

Anick Nater, MD; Lindsay Tetreault, Bsc; Branko Kopjar,
MD; Paul M. Arnold, MD; Mark Benedict Dekutoski; Charles Fisher, MD; Joel Finkelstein, MD; John France, MD; Ziya L. Gokaslan, MD; Laurence D. Rhines, MD; Peter Rose; Arjun Sahgal; James M. Schuster, MD, PhD; A

Introduction: Using prospective data rigorously collected, we aimed to: (1) identify key preoperative predictors of survival in adults treated surgically for a single metastatic epidural spinal cord compression (MESCC) lesion; (2) examine how these predictors relate to seven current prognostic scoring systems; and (3) perform the first full external validation of these scoring systems in accordance to the TRIPOD statement.

Methods: 142 surgical MESCC patients were enrolled in a prospective, multicenter, North American, cohort study and followed postoperatively for 12 months or until death. Cox proportional hazards regression was used following the assessment of the proportional hazards assumption. Non-collinear predictors of survival with $<10 \%$ missing data, $=10$ events per stratum and $\mathrm{p}<0.05$ in univariable analysis were tested through a backward stepwise selection process. Bootstrapping was used for optimism correction. Calibration was examined graphically and discrimination with Harrell c-statistics. Survival stratified by risk groups was evaluated using the Kaplan-Meier method and log-rank test.

Results: The median survival was 7.5 months. Eight factors were significant in univariable analysis: Tomita tumor grade, sex, visceral metastasis, body mass index, preoperative radiotherapy to MESCC, and SF-36v2 Physical Component (PC), EQ-5D and Oswestry disability index scores. Tomita tumor Grade II/III (HR: 2.767, 95\% CI: $1.520-5.035, \mathrm{p}=0.0009$ ), visceral metastasis (HR: 2.044, 95\% CI: $1.259-3.319, \mathrm{p}=0.0038)$, and SF-36v2 PC score (HR: $0.945,95 \%$ CI: $0.920-0.970, p<0.0001)$ were independently associated with survival; corrected discrimination was $0.68(95 \%$ CI: 0.66-0.70). Of the seven prognostic scoring systems, OSRI had the best discrimination $(0.68 ; 95 \%$ CI: 0.650 .71$)$.

Conclusion: Slow growing tumor, absence of visceral metastasis, and lower degree of patient-reported physical disability are independent preoperative predictors of longer survival in surgical MESCC patients. These results are in keeping with current prognostic scoring systems. This first full external validation of seven current prognostic scoring systems revealed that discrimination was at best fair.

295 Development and Validation of Clinical Prediction Models of Survival and Clinical Outcomes for Patients with Metastatic Epidural Spinal Disease: A Systematic Review

Anick Nater, MD; Jetan H. Badhiwala, MD; James Hong; So Kato; Melanie Anderson; David Choi, FRCS, ChB, PhD; Michael G. Fehlings, MD, PhD, FRCS(C), FACS

Introduction: In multivariable prognostic research, the development and external validation are the first phases typically involved towards the establishment of clinical prediction models (CPMs) in practice. This systematic review aims to identify and assess CPMs created to predict clinical outcomes in patients with metastatic epidural spinal disease (MESD) and subsequent validation studies.

Methods: Three electronic databases were searched (January 1, 1990 to June 20,2017), without language restriction, to identify studies that developed or evaluated CPMs predicting any clinical outcomes in adult patients with MESD (PROSPERO: CRD42017072908). Selected studies were then assessed based on their accordance with the Transparent Reporting of a multivariable prediction model for Individual Prognosis Or Diagnosis (TRIPOD) statement.

Results: Among 7,275 unique full-text articles, 106 were 
included. Among the 42 articles describing the development of a CPM, 25 did not include any assessment related to internal validity/model performance while 13 reported the number of outcome events and 5 how missing data were handled and only one allowed to obtain outcome predicted probabilities. We identified 79 studies evaluating CPMs. Among the 25 articles with the term "validation", "validated" or "validity" in the title or abstract, missing data for predictors and outcome, number of outcome events, and both calibration and discrimination were specifically mentioned in 8,15 and 3 studies, respectively.

Conclusion: Since 1990, over 40 CPMs predicting clinical outcomes in patients with MESD were developed and 76 studies performing some sort of evaluation of these CPM were published. Based on the items included in the TRIPOD statement, the majority of these studies did not report on key methodological and data analysis elements. The lack of rigor in the development and validation of CPMs may explain why most CPMs are not generally used in clinical practice.

296 Difference in Independent Patient Factors Associated with 30- and 90-Day Unplanned Readmission Rates After Elective Lumbar Spinal Fusion Versus Laminectomy: A National Trend Analysis of 152,426 Patients

Aladine A. Elsamadicy, BE; Hanna Kemeny; Xinru Ren, MS; Amanda Sergesketter; Eric W. Sankey, MD; C. Rory Goodwin, $\mathrm{MD}, \mathrm{PhD}$; Isaac O. Karikari, MD; Jichun Xie, PhD; Shivanand P. $\mathrm{Lad}, \mathrm{MD}, \mathrm{PhD}$

Introduction: The aim of this study was to investigate whether the modifiable patient risk factors associated with 30- and 90-day unplanned readmissions were similar or different between patients undergoing elective lumbar spinal fusion or decompression.

Methods: The National Readmission Database (NRD) was queried to identify all patients who underwent elective lumbar spinal fusion or decompression for the 2013 and 2014 calendar years. Patients were grouped by no readmission (Non-R), unplanned readmission within 30-days (30-R) and unplanned readmission within 31-90 days (90-R) of hospital discharge. Patient demographics, comorbidities, hospital characteristics, and 30- and 90 -day complication rates were collected. The primary outcome of interest was the rate of unplanned 30- and 90-day readmission rate and associated patient risk factors. A multivariate analysis was used to determine patient factors that were independently associated with unplanned 30- and 90-day readmission after elective lumbar spinal fusion and decompression.

Results: 152,426 adult patients were included in the study, $60,120(39.4 \%)$ patients had a lumbar spinal fusion (SF) and $92,336(60.6 \%)$ had a laminectomy \pm discectomy (LD). In the spinal fusion cohort, $4,371(7.3 \%)$ patients had an unplanned readmission, of which $3,067(5.1 \%)$ occurred within 30-days (30-R) and 1,304 (2.2\%) within 31-90 days (90-R) after hospital discharge. In the laminectomy \pm discectomy cohort, $6,849(7.4 \%)$ patients experienced an unplanned readmission, with 4,579 (5.0\%) within $30-\mathrm{R}$ and $2,270(2.5 \%)$ within $90-\mathrm{R}$ following hospitalization. In a multivariate regression analysis, insurance status (Medicare, Medicaid, and Self-Pay), COPD, depression, hypertension, diabetes, deficiency anemia, and obesity were independently associated with readmissions between both cohorts. While, age was only a predictor for 30-day readmission for spinal fusion patients.

Conclusion: study identified several patient demographics and modifiable comorbidities that were similar and independently associated with both 30- and 90-day unplanned readmissions for both fusion and decompression patients.

\section{Predictors of Patient Satisfaction in Spine Surgery: A Systematic Review}

Benjamin Yao, BA; Richard C Lee, BA; Jay M Levin, BA; Joseph E Tanenbaum, BA; Thomas E. Mroz, MD; Michael P. Steinmetz, MD

Introduction: The inclusion of patient satisfaction metrics in modern medical practice has increasingly influenced how physicians and hospital systems are evaluated. Despite several studies highlighting the importance of patient satisfaction with regards to optimizing clinical outcomes, the current spine surgery literature does not consistently report patient factors associated with patient satisfaction. Thus, the purpose of this study was to: 1) evaluate how patient satisfaction is measured in the spine surgery literature and 2) determine patient and surgical factors that are associated with patient satisfaction in this population.

Methods: A systematic literature search was performed to retrieve all studies that reported on patient satisfaction in the spine surgery setting. PubMed, Cochrane, and Web of Science Library electronic databases were initially queried, resulting in 965 studies. Two reviewers independently reviewed the studies for eligibility based on our inclusion criteria and included 32 studies in our final qualitative analysis. Study characteristics, patient satisfaction metrics, and all data measuring the association between patient factors and patient satisfaction were collected.

Results: Three major findings prevailed: (1) patient satisfaction measures varied widely, ranging from standardized Press Ganey Satisfaction surveys to simple binary satisfied/unsatisfied questionnaires; (2) patient satisfaction was commonly associated with psychological distress, high expectations for improvement, and post-operative pain; and (3) patient satisfaction was not consistently associated with medical comorbidities.

Conclusion: Our study identifies several important associations between patient factors and satisfaction that are consistently reported in the spine surgery literature. However, given the heterogeneous nature of patient satisfaction reporting and lack of consistency among several other associations between patient factors and satisfaction, the data also emphasizes the need for a more consistent definition of "patient satisfaction" and how it is measured in spine surgery.

298 Does Patient Selection Account for the Perceived Cost Savings in Outpatient Spine Surgery?

Benjamin Mundell, PhD; Marcus J. Gates, MD; Mohammed Ali Alvi, MD; Panagiotis Kerezoudis; Brett Arthur Freedman; Ahmad Nassr, MD; Samuel Hohmann, Ph.D; Anshit Goyal, MBBS; Mohamad Bydon, MD

Introduction: The perceived cost savings with comparable or improved outcomes to inpatient admission for the same procedures is desirable in an era where health expenditures are scrutinized. In this study, the authors' hypothesized that total savings in outpatient spine surgery is largely driven by patient selection and biases towards healthier patients.

Methods: A meta-analysis assessed patient selection factors and outcomes associated with outpatient spine procedures. Additional Bayesian analysis, informed by the meta-analysis results, was performed on a national administrative database to further compare inpatient and outpatient direct costs.

Results: Outpatient procedures were associated with younger patient age (MD $=-2.33,95 \% \mathrm{CI}:-4.40--0.25)$ and no diabetes diagnosis $(\mathrm{OR}=0.77,95 \% \mathrm{CI}: 0.51-0.97)$. Outpatient procedures were associated with a lower likelihood of reoperation $(\mathrm{OR}=$ 0.44, 95\% CI: 0.18-0.87), 30-day readmission (OR $=0.39,95 \%$ 
CI: 0.10-0.80), complication (OR $=0.29,95 \%$ CI: $0.15-0.49)$, and lower overall costs $(\mathrm{MD}=-\$ 13,834,95 \% \mathrm{CI}:-\$ 22,883--\$ 4629)$. Additional analysis of the national administrative data revealed more modest cost savings than those found in the meta-analysis for outpatient spine surgeries relative to inpatient spine surgeries. Estimated cost savings for both younger - $\$ 555$ for those age $30-35$ (95\% CI: - \$733 -\$374) - and older patients - $\$ 7290$ for those age $65-70$ (95\% CI: $-\$ 7380$ \$7190) were less than the overall cost savings found in the meta-analysis

Conclusion: Compared to inpatient surgery, outpatient spine surgery was associated with better short-term outcomes and an initial reduction in direct costs. A selection bias for outpatient procedures towards younger healthier patients may confound these results. The additional analysis of the national database suggests that cost savings in the outpatient setting may be less than previously reported and a result of outpatient procedures being offered more frequently to younger and healthier individuals.

299 Advanced Practice Provider Clinics Improve Patient Access in Neurosurgery Spine Clinics

Angela M Jolivette, APNP; Stephanie Stroud; Megan Bellman, ANSPA; Marjorie C. Wang, MD, MPH; Shekar N. Kurpad, MD, $\mathrm{PhD}$

Introduction: Prompt access to care is essential for positive patient experience. Access is defined as ability of a patient to get the correct care expeditiously, in the correct location, and with the correct provider. We evaluated barriers to patient access within a multidisciplinary spine center at a large tertiary health system in Milwaukee, WI. The objective of this study was to examine whether Advance Practice Provider (APP) based clinics could replace a pre-existing cumbersome review process while improving patient access without harming patient satisfaction.

Methods: We defined access as lag time between the date an appointment was made and the date the patient was evaluated by a member of the neurosurgery team. We reviewed monthly referrals from January to May 2016. Patient lag time was retrospectively analyzed. APP Access Clinics were conceived and implemented in late 2016. Subsequently, lag data were again analyzed after operationalizing APP access clinics over a 5 month period (January to May 2017).

Results: The initial mean lag time was 16.19 days. Implementation of measures for improvement, which included restructuring of a complex referral review process, staff education, creation of an intake questionnaire and significant expansion of the Spine APP role, improved lag time to 9.95 days. In addition to improved lag time, we found that APP based clinics did not negatively impact patient satisfaction scores. Further, APP access clinics improved the percentage of new patients seen by the surgeons who were surgical candidates.

Conclusion: APP based clinics significantly improved patient access time without negatively impacting patient satisfaction and optimized surgeon time by directing surgical versus non-surgical referrals appropriately. Further, optimized access to expeditious care might secure competitive advantage within the neurosurgery spine market.

300 Adverse Events and Their Risk Factors After Cervical Spine Surgery: Analysis from the Michigan Spine Surgery Improvement Collaborative (MSSIC)

Hesham Mostafa Zakaria, MD; Michael Bazydlo, MS; Shujie Xiao, MS; Lonni Schultz, PhD; David Nerenz, PhD; Muwaffak Abdulhak, MD, FRCS; Jason M. Schwalb, MD, FAANS, FACS; Victor W. Chang, MD; For the MSSIC Investigators
Introduction: The Michigan Spine Surgery Improvement Collaborative (MSSIC) is a statewide multicenter quality improvement collaborative.1 Using MSSIC data, we sought to identify 90-day adverse events after cervical spine surgery and their associated risk factors (RFs).

Methods: A total of 6542 cervical spine surgery cases were analyzed. Multivariate logistic regression models were constructed to identify RFs for adverse events; variables tested include age, gender, DM, disc herniation, foraminal stenosis, central stenosis, ASA $>2$, myelopathy, private insurance, house-hold income, outpatient vs inpatient procedures, anterior vs posterior approach, revision procedures, number of surgical levels, length of procedure, blood loss, preoperative ambulation, ambulation day of surgery, length of hospital stay, and disposition.

Results: 90d after cervical spine surgery, adverse events identified include radicular findings $(10.79 \%)$, readmission $(7.76 \%)$, urinary retention $(5.01 \%)$, UTI $(2.23 \%)$, dysphagia requiring NPO or feeding tube $(1.9 \%)$, surgical site hematoma (1.05\%), SSI $(0.83 \%)$, DVT $(0.68 \%)$, PE $(0.55 \%)$, neurogenic bowel/bladder $(0.48 \%)$, myelopathy $(0.44 \%)$, MI $(0.40 \%)$, claudication $(0.23 \%)$, ileus $(0.20 \%)$, and wound dehiscence $(0.18 \%)$. Male gender $(\mathrm{p}=0.016)$ and same-day ambulation $(\mathrm{p}=0.006)$ were protective of radicular findings. RF for dysphagia requiring NPO or feeding tube include anterior approach $(\mathrm{p}<0.001)$ and age $(\mathrm{p}<0.001)$, with protective factors including preoperative ambulation $(\mathrm{p}=0.037)$ and ambulation day of surgery $(p<0.001)$. RFs for readmission include ASA $>2(\mathrm{p}<0.001)$, while preoperative ambulation $(\mathrm{p}=0.008)$, private insurance $(\mathrm{p}=0.002)$, and being discharged home $(p<0.001)$ were protective. RFs for urinary retention include increasing age $(p<0.001)$, male gender $(p<0.001)$, increasing income $(p<0.001)$, and posterior approach surgery $(p<0.001)$, while preoperative ambulation $(\mathrm{p}=0.005)$ and ambulation day of surgery $(\mathrm{p}=0.002)$ were protective.

Conclusion: A multivariate analysis from a large, multicenter, prospective database identified the common adverse events after cervical spine surgery, along with their associated risk factors. This information can lead to more informed surgeons and patients. More detailed analysis is needed to identify anterior and posterior specific adverse events and their risk factors.

301 Predictors of Patient Satisfaction Survey Participation Following Spine Surgery: An Analysis of 17,853 Patients

Benjamin Hopkins, BS; Michael Cloney, MD; Tyler R. Koski, MD; Zachary A Smith, MD; Nader S. Dahdaleh, MD

Introduction: Patient satisfaction is an important metric for providers and hospitals alike. It is increasingly being tied to reimbursement, and through online ratings sites, may affect where patients seek care.1,2 Despite their importance, little is known about what factors predict participation in patient satisfaction surveys.

Methods: 17,853 consecutive spinal surgeries between at a single academic institution were retrospectively analyzed. Demographic, clinical, surgical and outcomes data were collected for each patient. Patients who completed Press Ganey or HCAHPS patient satisfaction surveys were identified. Analysis was performed to identify factors associated with completing a survey

Results: Of the 17,853 surgeries identified, $1936(10.8 \%)$ patients filled out Press Ganey surveys, while 2116 (11.9\%) patients filled out HCAHPS surveys. Patients were more likely to complete Press Ganey and HCAHPS surveys if they underwent a fusion $(\mathrm{p}<0.001$ for both), underwent thoracolumbar surgery ( $\mathrm{p}<0.001$ for both), had a VTE ( $\mathrm{p}=0.001$ and 0.003 respectively), had Acute Renal Failure ( $\mathrm{p}=0.026$, HCAHPS) or had a CSF leak 
$(\mathrm{p}=0.057$, HCAHPS). Patients were less likely to complete Press Ganey and HCAHPS surveys if they were male $(p<0.001$ for both), had an urgent/emergent procedure ( $\mathrm{p}<0.001$ for both), or had a longer length of stay ( $\mathrm{p}<0.001$ for both).

Conclusion: Women and patients undergoing certain types of procedures are more likely to complete Press Ganey and HCAHPS surveys. Patients who experience negative outcomes are more likely to complete surveys, while longer length of stay makes survey completion less likely. It is unlikely that patient satisfaction surveys provide a representative sample of the surveyed population.

302 A Longitudinal Survey of Adult Spine Case Entries During Neurosurgery Residency Training MD

Nitin Agarwal, MD; Michael D White, BS; D. Kojo Hamilton,

Introduction: The current study utilizes the American College of Graduate Medical Education case log system to analyze national trends in neurosurgical resident exposure to adult spinal procedures.

Methods: Prospectively populated ACGME resident case logs from 2013 to 2017 were retrospectively reviewed. The reported number of spinal procedures was compared to the ACGME minimum requirements for each surgical category pertaining to adult spine surgery. A linear regression analysis was conducted to identify changes in operative caseload by residents graduating during the study period, as well as a one sample t-test using IBM SPSS software (Armonk, NY) to compare the mean number of procedures in each surgical category to the ACGME required minimums.

Results: A mean of 427.42 total spinal procedures were performed throughout residency training for each of the 877 residents graduating between 2013 and 2017. The mean number of procedures completed by graduating residents increased by 19.96 (r2 = 0.95 ) cases per year, with every surgical category increasing during this time. The two surgical categories with the largest changes were anterior and posterior cervical approaches for decompression/stabilization, which increased by $8.78 \%$ per year $(\mathrm{r} 2=0.95)$ and $9.04 \%$ per year $(\mathrm{r} 2=0.95)$ respectively. It was also found that the percentage of total spinal procedures performed during chief year fell from $83.70 \%$ in 2013 to $36.56 \%$ in 2017 , despite an overall increase in spinal procedures. Residents' mean caseloads during residency were found to be vastly greater than the ACGME required minimums: residents performed at least twice as many procedures as the required minimums in every surgical category.

Conclusion: Graduating neurosurgical residents reported increasing case volumes for adult spinal cases during this fiveyear interval. The average resident was noted to perform more than twice the number of procedures required by the ACGME, revealing that neurosurgical residents are achieving more exposure to spine surgery than expected.

303 Morbidity and Financial Burden Associated with Reoperation After Lumbar Discectomy: Results from a Study Evaluating Annular Closure

Michael W. Groff, MD, FACS; Claudius Thome, MD; Wellington Hsu, MD; Joel Batts, MA; Frederic Martens, MD

Introduction: The purpose of this study was to examine the outcomes and US costs of reoperation following primary discectomy, with or without an annular closure device (ACD) in patients at highest risk of reherniation.

Methods: This is a post-hoc analysis of a multicenter randomized controlled trial (RCT) comparing an ACD to conven- tional lumbar discectomy (Control) in patients with annular defect widths $>6 \mathrm{~mm}$. All 550 patients from the RCT were included (69 reoperated; 481 non-reoperated). Patients were divided into 4 cohorts based on intervention (Control or ACD), and reoperated or non-reoperated (regardless of intervention) within 24 months. Follow-up visits were 6 weeks through 24 months after index procedure for all cohorts. Reoperations included any surgery at the index level. Outcome measures were VAS leg/back, Oswestry Disability Index (ODI), mental (MCS) and physical composite scores (PCS) of the Short Form-36, working status, missed work, physiotherapy time, and number of inpatient days due to serious adverse event. Literature-derived US payer costs were applied to direct and indirect variables.

Results: At 24 months, reoperated patients had significantly worse scores for ODI, VAS leg/back, and MCS/PCS scores compared to non-reoperated $(\mathrm{p}<0.0001)$. The rate of reoperated versus non-reoperated patients who did not improve clinically was 2.9 times higher based on ODI (46\% vs. 16\%). Per patient costs were $\$ 69,484$ in the reoperated group compared with $\$ 9,697$ for non-reoperated through 24 months. In the ACD and Control groups, 24/267 (9\%) and 45/283 (16\%) patients underwent indexlevel reoperation $(\mathrm{p}=0.01)$. Mean estimated cost per Control $(\$ 22,982)$ was $13.5 \%$ greater than $\operatorname{ACD}(\$ 18,088)$ after accounting for device cost.

Conclusion: Reoperation after index lumbar discectomy results in significant patient morbidity and costs in this high-risk population. ACD reduced the rate of reoperation by $44 \%$ and per patient costs by $13.5 \%$. Annular closure is worthy of consideration for addressing this challenging population.

\section{Device}

304 Direct Cost Analysis of a Novel Annular Closure

Jared Ament, MD, MPH; Bart Thaci, MD; Rakesh Patel, MD; Matthew J. McGirt, MD; Gerrit Joan Bouma, MD; Jason Cuellar, MD, PhD; Todd Hopkins Lanman, MD; Kee Duk Kim, MD; J. Patrick Johnson, MD, MS

Introduction: Lumbar discectomy is a largely successful surgical procedure; however, reoperation rates in patients with large annular defects are as high as $27 \%$. The costs associated with revision discectomy surgery places a significant burden on the healthcare system. We compared the direct costs of conventional discectomy (Control) with those of discectomy with a novel annular closure device (ACD).

Methods: All-cause index level reoperations were reviewed from a multicenter randomized controlled trial (RCT) that allocated 554 high risk reherniation patients to either Control or ACD groups. Medicare and private insurer direct costs were derived from a commercially available payer database, including those associated with facility, surgeon, imaging, follow-up visits, physical therapy, and injections. Costs were then applied to each instance of reoperation, with all care settings using inpatient costs, except for repeat discectomy which used a 60/40 inpatient-tooutpatient ratio. Total costs of reoperation and per patient costs of reoperation were compared between groups for both forms of insurers.

Results: Use of the ACD reduced the rate of reoperations to $9 \%(n=276)$ compared to $22 \%(n=278)$ in the control group $(\mathrm{p}=.001)$. Total Medicare costs incurred for all-cause reoperation were nearly double for Controls versus ACD $(\$ 824,000$ versus $\$ 436,000)$ as well as private insurer $(\$ 1.3 \mathrm{M}$ versus $\$ 703,000)$. Perpatient costs incurred were $\$ 1385$ and $\$ 2110$ greater for Controls for Medicare and private insurer, respectively.

Conclusion: At two years follow up, the total directs costs of reoperation was nearly double for Controls versus ACD for both 
Medicare and private insurance types. This difference resulted in increased per-patient costs as high as $\$ 2110$ for all Controls versus ACD patients. The reduced reoperation rate observed with the novel ACD results in concomitant cost reductions within two years of index operation.

305 Obesity is Associated with Inferior Patient Reported Outcomes Following Surgery for Degenerative Lumbar Spondylolisthesis: An Analysis of the Quality Outcomes Database

Andrew Kai-Hong Chan, MD; Erica Fay Bisson, MD, MPH, FAANS; Mohamad Bydon, MD; Steven D. Glassman; Kevin T. Foley, MD, FACS, FAANS; Eric A. Potts, MD; Christopher I. Shaffrey, MD, FACS; Mark Edwin Shaffrey, MD, FAANS, FACS; Domagoj Coric, MD; John J. Knightly, MD;

Introduction: In light of the recent differing findings following two randomized clinical trials on surgery for degenerative lumbar spondylolisthesis (DLS), there is a need for the identification of patients who may benefit least versus most from surgery. This study investigates the impact of obesity on patient reported outcomes (PROs) following surgery for DLS.

Methods: Eleven of the highest-enrolling sites were queried and we found 477 patients undergoing elective spine surgery for degenerative grade 1 lumbar spondylolisthesis. For univariate comparisons, patients were stratified by $\mathrm{BMI}=30 \mathrm{~kg} /$ $\mathrm{m} 2$ (obese) and $<30 \mathrm{~kg} / \mathrm{m} 2$ (non-obese). Baseline, 3-month, and 12-month follow-up parameters were collected. PROs included the North American Spine Society (NASS) satisfaction questionnaire, numeric rating scale (NRS) back pain, NRS leg pain, Oswestry Disability Index (ODI), and EuroQoL-5D (EQ-5D).Questionnaire.

Results: We identified 224 obese $(47.0 \%)$ and 253 nonobese patients $(53.0 \%)$. Obese patients more often presented with sensory predominant symptoms $(46.4 \%$ vs $38.9 \%, \mathrm{p}=0.02)$ and were less independently ambulatory $(82.5 \%$ vs. $93.7 \%, \mathrm{p}<0.01)$. Obese patients more often underwent fusion surgery $(87.9 \%$ vs $78.3 \%, \mathrm{p}<0.01)$, had higher estimated blood loss $(302.9 \pm 327.5$ vs $213.3 \pm 227.0 \mathrm{ml}, \mathrm{p}<0.01)$, longer operative times $(212.7 \pm 95.2 \mathrm{vs}$ $177.2 \pm 80.4 \mathrm{~min}, \mathrm{p}<0.01)$, and longer hospitalizations $(3.3 \pm 1.6$ vs $2.9 \pm 2.0$ days, $\mathrm{p}<0.01)$. At baseline, obese patients had worse NRS back pain, ODI, and EQ-5D scores $(p<0.05)$. Both cohorts improved significantly from baseline for back and leg pain, ODI, and EQ-5D at 12 months $(\mathrm{p}<0.01)$. At 12 months, fewer obese patients responded that surgery met their expectations $(64.4 \%$ vs. $70.1 \%, \mathrm{p}<0.01)$. In adjusted multivariate analyses, BMI was independently associated with worse NRS leg pain, ODI, and EQ-5D at 12 months $(\mathrm{p}<0.05)$.

Conclusion: Obesity is independently associated with inferior pain, disability, and quality of life 12 months post-operatively. Surgeons may want to discuss these findings with obese patients to help set appropriate expectations.

306 Reductions in Length of Stay and Blood Loss With Standardized Care by Neuro-anesthesiologists

Ahilan Sivaganesan, MD; Inamullah Khan, MD; Letha Matthews, MD; Byron Fitzgerald Stephens; Clinton J. Devin, MD

Introduction: The benefits of standardizing surgical practices have been well-delineated for many fields. The dividends of applying this mindset towards care by anesthesiologists, however, are less understood. Here we present outcomes before and after instituting standardized care by neuro-anesthesiologists for all elective lumbar surgeries at our institution.

Methods: A prospective registry was used to collect patient- and surgery-specific variables, as well as the following outcomes, before and after implementation of standardized care by neuro-anesthesiologists: length of stay (LOS), discharge to home, readmission, complications, and estimated blood loss (EBL). The protocol involved vigilant blood pressure control, judicious use of anesthetics to enable quick post-operative emergence, normalization of body positioning/padding, and the routine use of intraoperative NSAIDs such as Ketorolac. A fixed set of anesthetists was also established for all surgeries. The Mann-Whitney U test and Chi-squared test were used to make comparisons between the two cohorts. Multivariable proportional odds and logistic regression models were fit to evaluate the risk-adjusted impact of standardization on outcomes.

Results: Our study cohort consists of 2744 pre-standardization and 256 post-standardization patients. The latter group had some systematic differences, such as being slightly younger, less insured, less medically complex (ASA grade), and undergoing operations of reduced duration on fewer spinal levels (Table 1). Significant testing showed significant reductions in LOS and EBL with the post-standardization group $(\mathrm{p}<0.001)$, but no difference with respect to the other outcomes (Table 2). The same results were seen with risk-adjusted analysis using multivariable regression (Tables 3,4). Interactions between the use of fusion procedures and standardization were incorporated.

Conclusion: LOS and EBL for elective lumbar surgery improved after implementation of a standardized peri- and intraoperative protocol by neuro-anesthesiologists at our institution. Efforts such as this, focusing not only on surgical practices but also anesthesiology care, can improve the value of spine care.

307 High Risk Neurosurgical Spine Patients are Associated with Increased In-hospital Costs

Sasha Vaziri, MD; Joseph Abbatematteo; Paul Kubilis, MS; Daniel J. Hoh, MD

Introduction: The American College of Surgeons (ACS) National Surgical Quality Improvement Program (NSQIP) online Surgical Risk Calculator uses inherent patient characteristics to provide predictive risk scores for adverse postoperative events. The purpose of our study is the determine if neurosurgical spine patients with a high predicted risk scores are associated with increased in-hospital costs.

Methods: A single-center retrospective review of 192 neurosurgical spine patients treated in the period from September 2011 through December 2014 was performed. Individual patient characteristics were entered into the NSQIP calculator. Predicted risk scores were compared with actual in-hospital costs obtained from a billing database. Statistical models were used to determine if patients with higher risk scores were associated with increased in-hospital costs.

Results: We used the pearson correlation coefficient $(\mathrm{R})$ to assess correlation between 11 types of complication risk scores and 5 types of encounter costs from 192 spine UF health encounters involving spine procedures. Risk scores in categories such as serious complication, any complication, pneumonia, cardiac complication, surgical site infection, urinary tract infection, venous thromboembolism, renal failure, return to operating room, death, and discharge to nursing home or rehab were examined. Patients with higher risk scores were found to have a statistically significant association with increased in-hospital costs $(p<0.05)$.

Conclusion: Previous work has demonstrated the ACS NSQIP Surgical Risk Calculator can accurately predict mortality but is poorly predictive of other potential adverse events and clinical outcomes. However, this study demonstrates that high-risk patients identified by the ACS NSQIP Surgical Risk Calculator have a sta- 
tistically significant association with an increase in-hospital cost. The NSQIP calculator may not accurately predict surgical risk (as demonstrated previously), given the current healthcare climate future iterations of the ACS Universal Risk Calculator may be valuable tool in predicting in-hospital costs.

308 Lumbar Spine Fusion: The Influence of Evidence on Decision Making

Daniel Yavin, MD; Steven Casha, MD, PhD; Samuel Wiebe, MD, MSc; Thomas E. Feasby, MD; R. John Hurlbert, MD, PhD, FACS, FRCS(C); Andrew Nataraj, MD; Garnette R. Sutherland, MD, FRCSC; Perry Pawandeep Singh Dhaliwal, MD; Stephan Jean du Plessis; Nathalie Jette

Introduction: Evaluation of the determinants of widely varying regional rates of lumbar fusion is needed. While patient, surgeon, and setting-specific factors have been investigated; the association of between decision making and the quality of the underlying evidence remains unclear. This study therefore aimed to evaluate the influence of the quality of evidence on experts' decision to perform lumbar fusion for degenerative indications.

Methods: The quality of evidence underlying 1296 distinct degenerative indications for lumbar fusion was graded according to criteria developed by the North American Spine Society. A North American expert panel of 13 physicians was convened and members were provided with scenario-specific evidence grades and a systematic literature review evaluating the safety and efficacy of lumbar fusion. Using a modified Delphi process, panel members rated the appropriateness of lumbar fusion for each indication. The strength of association between expert panel disagreement and scenarios' clinical characteristics, radiologic findings, and levels of evidence was assessed.

Results: In the 1296 scenarios evaluated, $13 \%$ were supported by level I evidence, $33 \%$ by level II evidence, and $54 \%$ by level III evidence. Indications involving spondylolisthesis were associated with the highest percentage of level I evidence (Fig. 1). Increasing quality of evidence was not associated with decreasing disagreement when panelists considered lumbar fusion for spinal stenosis $(\mathrm{P}=0.11)$, spondylolisthesis $(\mathrm{P}=0.79)$, disc herniation $(\mathrm{P}=1.0)$, or spondylosis ( $\mathrm{P}=0.63)$ (Fig. 2). In contrast, disagreement among panelists was dependent upon the extent of patients' disability $(\mathrm{P}<0.001)$, form of low back pain $(\mathrm{P}<0.001)$, severity of neurologic signs and symptoms $(\mathrm{P}<0.001)$, and radiologic findings $(\mathrm{P}<0.001)$ (Fig. 3).

Conclusion: The quality of underlying evidence for lumbar fusion did not account for differing surgical decision making. The reliance on level III evidence for the majority of surgical indications may limit the use of the available literature in patient care decisions. (Funded by the Alberta Spine Foundation.)

309 Effectiveness of a Decision-making Protocol for the Surgical Treatment of Lumbar Stenosis with Grade 1 Degenerative Spondylolisthesis

\section{Victor E. Staartjes, BS; Marc L. Schröder, MD, PhD}

Introduction: The addition of fusion to decompression for stenosis with Grade I degenerative spondylolisthesis is a controversial topic, and the question remains if fusion provides any real benefit to the patient that warrants the increased healthcare utilization and perioperative morbidity. In addition, there is no consensus on indications for the use of fusion over decompression alone. We aimed to assess the effectiveness of a decision-making protocol that assigns patients with stenosis and Grade 1 degenerative spondylolisthesis to either fusion surgery or decompression alone.
Methods: Patients received fusion or decompression according to a decision-making protocol based on their pattern of complaints, the location of the compression, as well as facet angles and effusion as proven predictors of postoperative instability. Using propensity scores, patients were matched demographic parameters and for baseline numeric rating scale (NRS) back pain, NRS leg pain, and Oswestry Disability Index (ODI). All data were collected in a prospective database. Patients were followed up at 6 weeks, 12 months and 24 months.

Results: 102 patients were included in two equally-sized groups. No intergroup differences in NRS and ODI were detected at the 6 week, 12 months, and 24 months follow up visits (all p $>0.05$ ). Duration of surgery, length of stay, estimated blood loss, and radiation doses were higher in the fusion group (all $p<0.001$ ). The cumulative reoperation rate was similar with $6 \%$ and $8 \%$ for fusion and decompression, respectively $(\mathrm{p}>0.05)$, as was the complication rate $(8 \%$ vs. $6 \%, p>0.05)$. Postoperative, iatrogenic progression of spondylolisthesis requiring fusion surgery was seen in only $2 \%$ in the decompression group.

Conclusion: Reoperations, complications and outcome measures were equal between fusion and decompression at all follow up lengths, and fusion procedures were associated with a significantly higher perioperative burden for the patient. The rate of reoperation for iatrogenic progression of spondylolisthesis after decompression alone was comparatively low at $2 \%$. These data suggest that a decision-making protocol based on the clinical history, location of the nerve root compression, and proven radiological predictors of postoperative instability assigns patients to fusion or decompression in a safe and effective manner.

310 Patient Rated Outcomes the Difference Between Satisfaction with Care and Perception of Surgical Outcome.

\section{Ben Z. Roitberg, MD}

Introduction: Patient ratings of care and outcome are increasing in importance as greater emphasis is placed on quality of care. Questionnaires of satisfaction with care were criticized for not representing the surgical outcome and for being a poor tool to evaluate the the performance of the surgical team. In this study, we analyze and compare patient satisfaction with care, with their perception of surgical outcome.

Methods: 1400 patients were prospectively enrolled in a operative spine outcome registry at the University of Chicago; 599 patients for whom at least two years of follow up was expected by $3 / 8 / 2016$ were included in this analysis.

Results: Response rate was $86 \%$ at 3 months, $85 \%$ at 6 months and $82 \%$ at 12 months postoperatively. There were 303 females, 296 males; average age was 58.78 years. 388 had lumbar disease and 211 had cervical spine disease. Satisfaction with care and positive perception of outcome demonstrated a significant correlation $(\mathrm{r}=0.67 ; \mathrm{p}<0.0001)$. However, there were patients with divergent opinions: $5.49 \%, 7.20 \%$ and $9.80 \%$ were satisfied with outcome but not care, whereas $3.14 \%, 3.31 \%$ and $2.45 \%$ percent were satisfied with care but not outcome, at 3,6 and 12 months respectively. Perceived poor outcome at three months was associated with loss to follow up at 12 months. Of 16 patients who at three months felt that they did not improve or became worse after the operation, 14 dropped out and only 2 returned the follow up forms at 12 months. Among those who perceived a good outcome but bad care, 20 out of 28 dropped out.

Conclusion: Most patients were satisfied with their care and had a positive perception of outcome. Patients may have divergent opinions regarding their outcome and the quality of their care. Poor outcome or dissatisfaction with care lead to loss to follow up. 
311 Clinical Outcomes Research in Lumbar Spine Surgery: Are 2 year follow-ups Necessary?

Oliver GS Ayling, MD, MSc; Charles Fisher, MD; Tamir Ailon; Neil Manson, MD, FRCS(C); Raja Y. Rampersaud, MD, FRCS(C); Nicolas Dea, MD, MSc, FRCSC

Introduction: There has been generic dictum in spine and musculoskeletal clinical research that 2 year follow-up is necessary for patient reported outcomes (PRO) ; however, the rationale for 2 year follow-up is not evidence based. Therefore the purpose of this study is to determine the PRO follow-up time necessary to ensure the effectiveness of a lumbar surgical intervention is appropriately evaluated.

Methods: Using PROs from the Canadian Spine Outcomes and Research Network (CSORN) prospective database the time-course to plateaued recovery after lumbar spine surgery was assessed for lumbar disc herniation, degenerative spondylolithesis, and spinal stenosis. One-way ANOVAs with post-hoc testing were used to analyse PROs at baseline, three, twelve, and twenty-four months post-operatively on the following standardized PROs: EQ5D, ODI, VAS leg and back, and SF-12 mental and physical scales.

Results: There were significant differences determined by one-way ANOVAs for all spine pathologies and specific PROs $(\mathrm{p}<0.0001)$. Time to plateaued recovery after surgery for lumbar disc herniation, lumbar spondylolithesis, or lumbar stenosis followed the same time-courses for the following PROs: VAS back and leg 3 months, ODI 12 months, SF-12 physical 12 months, and SF-12 mental 3 months.

Conclusion: Individual PROs after surgery for lumbar spine pathologies follow specific time-courses to plateaued recovery indicating a two-year follow-up is not required for all outcomes to be accurately assessed. Ultimately the clinical research question should dictate follow-up time and the outcome measure utilized, however there is now evidence to guide the specific duration of follow-up for each PRO.

313 Reduced Impact of Smoking Status On 30-Day Complication and Readmission Rates After Elective Spinal Fusion (e"3 Levels) for Adult Spine Deformity: A Single Institutional Study of 839 Patients

Aladine A. Elsamadicy, BE; Owoicho Adogwa, M.D., M.P.H; Amanda Sergesketter; Victoria D Vuong; Emily Lydon, B.S.; Shay Behrens, B.S.; Joseph S. Cheng, MD, MS; Carlos Antonio Bagley, MD; Isaac O. Karikari, MD

Introduction: Smoking status has been shown to affect postoperative outcomes after surgery. The aim of this study is to determine if patients' smoking status impacts 30-day complication and re-admission rates after elective complex spinal fusion (=3 levels).

Methods: The medical records of 839 adult spinal deformity patients undergoing elective complex spinal fusion ( $=3$ levels $)$ at a major academic institution from 2005 to 2015 were reviewed. We identified $124(14.8 \%)$ smokers and 715(85.2\%) non-smokers (Smokers: $n=124$; Non-Smokers: $n=715$ ). Patient demographics, comorbidities, intra-operative and post-operative complication and 30-day readmission rates were collected for each patient. The primary outcome investigated in this study was the rate of 30-day postoperative complication and readmission rates.

Results: Patient demographics and comorbidities were similar between both groups, including age, gender, and BMI. Median [IQR] number of fusion levels and operative time were similar between the cohorts (Smoker: 5[4-7] vs. Non-Smoker: 5[4-8],p=0.58) and (Smoker: 309.6 \pm 157.9 mins vs. Non-Smoker: 287.5 \pm 131.7 mins, $\mathrm{p}=0.16$ ), respectively. Both cohorts had similar postoperative complication rates and lengths of hospital stay. There was no significant difference in 30-day readmission between the cohorts (Smoker: $12.9 \%$ vs. Non-Smoker: $10.8 \%, \mathrm{p}=0.48)$. There were no observed differences in 30-day complication rates, including pain $(\mathrm{p}=0.46)$, $\mathrm{UTI}(\mathrm{p}=0.54)$, hardware failure $(\mathrm{p}=0.36)$, wound dehiscence $(\mathrm{p}=0.29)$ and wound drainage $(\mathrm{p}=0.86)$. Smokers had higher rates of 30-day cellulitis (Smoker: $1.6 \%$ vs. Non-Smoker: $0.3 \%, \mathrm{p}=0.05$ ) and DVT (Smoker: $0.8 \%$ vs. Non-Smoker: $0.0 \%, \mathrm{p}=0.02$ ).

Conclusion: Our study suggests that smoking does not significantly affect 30-day readmission rates after complex spinal surgery requiring $=3$ levels of fusion. Further studies are necessary to corroborate our findings.

314 Predictors of Patient Satisfaction Following Spine Surgery: An analysis of $\mathbf{2 9 0 5}$ Patients from a Single Academic Institution

Benjamin Hopkins, BS; Jonathan Tad Yamaguchi, BS; Michael Cloney, MD; Zachary A Smith, MD; Tyler R. Koski, MD; Nader S. Dahdaleh, MD

Introduction: Patient satisfaction scores continue to gain more importance for both clinical practice and reimbursement. Currently, Press Ganey and the Hospital Consumer Assessment of Healthcare Providers and Systems (HCAHPS) are standardized surveys used to assess patient satisfaction, however little data exists to predict factors that lead to better satisfaction.

Methods: 2905 patients undergoing spine surgery at a single institution who subsequently filled out either a Press Ganey or HCAHPS survey were retrospectively analyzed. Subsequent demographic, surgical and outcome data were collected for each patient.

Results: Demographically, the majority of the patients were Caucasian $(76 \%)$ and were female $(53 \%)$. On multivariate analysis, patients were less likely to give a perfect score on both Press Ganey and HCAHPS surveys with increasing Charlson Comorbidity Index (OR 0.966, p=0.069; OR 0.932, $\mathrm{p}=0.012$ ). Individually, patients were less likely to give a perfect score as elapsed time since surgery and discharge increased (OR 0.996, $\mathrm{p}=0.083$, OR 0.642, $\mathrm{p}=0.054$ Press Ganey and HCAHPS respectively), length of stay increased (OR 0.940, $\mathrm{p}=0.002$ HCAHPS) and if their surgeon underwent a posterior approach $(\mathrm{OR} 0.810, \mathrm{p}=0.088)$. Revision trended towards significance (OR $0.642, p=0.054$ HCAHPS). Increasing length of stay and increasing elapsed time since surgery predicted receiving an overall score of 2 or less $(\mathrm{p}<0.001$ and 0.048 respectively HCAHPS), while a posterior approach was likewise protective of receiving low scores ( $\mathrm{p}=0.046$ Press Ganey). Surgeon, race, gender, region of the spine, urgent/emergent procedures and type of surgery were not predictive of changes in satisfaction scores.

Conclusion: Length of stay along with elapsed time between hospitalization and survey completion date may be influential predictors of patient satisfaction scores, with increasing times leading to worse scores. Similarly, higher comorbidity scores, having a revision surgery and having an urgent/emergent surgery may further predict lower patient satisfaction scores after spine surgery.

315 Independent Associations with 30- and 90-Day Unplanned Readmissions After Elective Lumbar Spine Surgery: A National Trend Analysis of 144,123 Patients

Aladine A. Elsamadicy, BE; Xinru Ren, MS; Hanna Kemeny; Lefko Charalambous, BS; Shervin Rahimpour, MD; Theresa Williamson, MD; C. Rory Goodwin, MD, PhD; Muhammad M Abd-El-Barr, MD, PhD; Oren N. Gottfried, MD; Jichun Xie, PhD; Shivanand P. Lad, MD, PhD

Introduction: There is a paucity of data identifying modifi- 
able patient risk factors that are independently associated with 30- and 90-day readmissions. The aim of this study was to investigate whether the modifiable patient risk factors associated with 30-day unplanned readmissions were similar to 90-day unplanned readmissions following elective lumbar-spine surgery.

Methods: The National Readmission Database (NRD) was queried to identify all patients who underwent elective lumbarspine surgery for the 2013 and 2014 calendar years. Patients were grouped by no readmission (Non-R), unplanned readmission within 30-days (30-R) and unplanned readmission within 31-90 days (90-R) of hospital discharge. Patient demographics, comorbidities, hospital characteristics, and 30- and 90-day complication rates were collected. The primary outcome of interest was the rate of unplanned 30- and 90-day readmission rate and associated patient risk factors. A multivariate analysis was used to determine patient factors that were independently associated with unplanned 30- and 90-day readmission after elective lumbar-spine surgery.

Results: We identified 144,123 patients who underwent elective lumbar-spine surgery with $10,592(7.3 \%)$ patients experiencing an unplanned hospital readmission (30-R: $n=7,228$ [5.0\%], 90-R: $n=3,364$ [2.3\%], Non-R: $n=133,531)$. Baseline patient demographics, comorbidities and hospital characteristics were similar between both cohorts. Spinal fusion was the most common spinal procedure with the highest readmission rates (30-R: 42.4\%, 90-R: $38.8 \%$ ). The most common inpatient complication observed was dural tear (30-R: $7.7 \%$, 90-R: $4.6 \%$, Non-R: $4.3 \%$ ). The most prevalent 30- and 90-day complication was infection (30-R: $18.5 \%, 90-\mathrm{R}: 7.4 \%$ ). In a multivariate regression analysis, age, insurance status (Medicare, Medicaid, and Self-Pay), COPD, depression, hypertension, diabetes, deficiency anemia, and obesity were independently associated with 30-day unplanned hospital readmission; while, age and obesity were not independently associated with 90-day unplanned hospital readmission.

Conclusion: Our study identified several patient demographics and modifiable comorbidities that were similar and independently associated with both 30 - and 90-day unplanned readmissions.

316 Superiority Claims Derived From Investigational Device Trials in the Spine: Grounds for Confidence or Concern? DO

Michael W. Groff, MD, FACS; Joel Batts, MA; Ali Araghi,

Introduction: Many non-inferiority trials of investigational devices proceed to post-hoc tests for statistical superiority once non-inferiority has been substantiated. Nuances associated with this strategy make interpretation of the superiority claim difficult. Superiority trial designs define success criteria a priori and effect size can be readily interpreted, especially when minimum clinically important differences (MCID) are used. We analyzed peerreviewed literature of investigational spine device trials that were published since 2000 to identify trial designs, superiority claims, and use of MCIDs.

Methods: A PubMed search was conducted using the search terms 'spine AND (investigational device exemption OR IDE)' between 2000 and 2017. The search was narrowed to only include articles in English and those providing details on the study design with follow-up of at least 2 years. Single arm studies were not included. Resulting articles were reviewed for study design, whether superiority was concluded and use of MCID.

Results: The initial search returned 192 publications related to IDE studies. There were 22 unique studies (40 publications) that met the inclusion/exclusion criteria. Of those 22 IDE studies, 21 (95\%) were non-inferiority designs and $1(5 \%)$ was a superiority design. Fourteen (64\%) of the non-inferiority studies made superi- ority conclusions. In all 14 of those studies, the superiority claim was solely based on statistical difference between the experimental and control groups, without any criteria for MCID or discussion of the clinical significance of the effect size.

Conclusion: Spine studies rarely employ superiority designs. We urge researchers to consider superiority trial designs using a priori definitions of superiority that are clinically relevant, especially when evaluating novel technologies against standard of care. In this way, health care providers and payers will make evidence-based decisions with greater confidence in the reported clinical outcomes.

317 Increased length of Stay Beyond Expected are Associated with Lower Hospital Consumer Assessment of Healthcare Providers and Systems Scores.

Gabriel Alexander Smith, MD; Jay M Levin, BA; Robert Winkelman, BS; Joseph E Tanenbaum, BA; Thomas E. Mroz, MD; Michael P. Steinmetz, MD

Introduction: Hospital Consumer Assessment of Healthcare Providers and Systems (HCAHPS) surveys are used to assess the quality of the patient experience following an inpatient stay. Recent studies have shown that HCAHPS scores may be influenced by preoperative or postoperative factors related to the patient and their care.

Methods: A total of 391 patients who underwent lumbar spine surgery who completed the HCAHPS survey between 2013 and 2015 at a single tertiary care center were included in a retrospective analysis. Patients were divided into two groups: those who had a length of stay in the hospital shorter or equal to their expected length of stay determined by University Health Consortium benchmarks and a longer than expected length of stay. The primary outcomes of this study were comparing top-box scores of "9-10" or "always or usually" versus low-box scores of "1-8" and "somewhat or never" on doctor and nursing questions respectively. Statistical analysis included Pearson chi-square for categorical variables.

Results: A total of 257 patients had a length of stay less than expected versus 134 patients who had a length of stay beyond expected. Only hypertension $57.8 \%$ versus $45.2 \%$ ( $\mathrm{p}=0.026$ ) was higher in patients with longer than expected LOS. Upon analysis, satisfaction with nursing explanations, listening, and respect were not statistically significant between groups. For doctors, patients with a longer than expected length of stay gave poorer ratings for doctor listening and respect during their stay. There was no difference in doctor explanations.

Conclusion: Our results demonstrate a strong association between longer than expected length of stay and patient satisfaction with physicians and how they're treated. Further studies are needed to identify patients at high risk for prolonged LOS and improved the quality of their care during admission.

318 Enhanced Recovery After Surgery (ERAS) Protocol in Spinal and Peripheral Nerve Surgery Improves Postoperative Mobility and Ambulation

Zarina S Ali, MD; Lena Leszinsky; Brendan McShane, BA; Tracy Ma Flanders, MD; Ali Kemal Ozturk, MD; Neil R. Malhotra, MD; Diana Gardiner, DNP, CRNP; Mara Cappelloni, NP; Kristin Rupich, NP; Emily NP Missimer; James M. Schuster, MD, PhD; Paul J. Marcotte, MD; M. Sea

Introduction: Enhanced Recovery after Surgery (ERAS) protocols are well established in many surgical subspecialties, but are limited in the neurosurgical population. The purpose of this study 
is to investigate the effects on postoperative mobilization and foley catheter usage after implementation of an ERAS protocol.

Methods: An ERAS protocol was developed and implemented in the Pennsylvania Hospital Department of Neurosurgery. The protocol included the need to mobilize patients within six hours postoperatively in the absence of bed rest precautions. Postoperative foley catheter use was limited to patients with bed rest precautions or spinal fusion $>3$ levels. The first 201 consecutive patients who underwent elective spinal or peripheral nerve surgery were enrolled in our study and compared to a similar historical cohort $(n=74)$ at the same institution, prior to initiation of the ERAS pathway. All emergency surgery cases were excluded. Patient mobility, ambulation, and foley catheter use were prospectively collected and analyzed using a Fisher's Exact test

Results: Mean patient age $(\mathrm{p}=0.13)$, gender $(\mathrm{p}=0.59)$, and distribution of surgical procedures $(\mathrm{p}=0.43)$ were similar between both groups. On the day of surgery, $53.4 \%$ of ERAS patients were mobilized after surgery compared to $17.1 \%$ of control patients $(p<0.001)$. At one day post-surgery, $84.1 \%$ of ERAS patients were mobilized compared to $45.7 \%$ control patients $(\mathrm{p}<0.001)$. Similarly, $46.9 \%$ of ERAS patients ambulated on the day of surgery compared to $17.1 \%$ of control patients $(\mathrm{p}=0.001)$ and $64.2 \%$ of ERAS patients ambulated one day post-surgery compared to $28.6 \%$ of control patients $(\mathrm{p}<0.001)$. In the postoperative setting, $20.4 \%$ of ERAS patients had a Foley catheter in place compared to $47.3 \%$ of control patients $(\mathrm{p}<0.001)$.

Conclusion: Substantial improvement in early postoperative mobility and ambulation can be gained with the introduction of an ERAS protocol. Future analysis will explore the impact of early mobilization within a ERAS protocol on hospital length of stay.

319 The Institute for Healthcare Improvement: NeuroPoint Alliance Cooperative Quality Improvement Project: Using National Registries to Design Continuous Quality Improvement Protocols

Vincent Rossi, MD; Scott L. Zuckerman, MD; Clinton J. Devin, MD; Kevin T. Foley, MD, FACS, FAANS; John J. Knightly, MD; Erica Fay Bisson, MD, MPH, FAANS; Steven D. Glassman; Anthony L. Asher, MD, FACS

Introduction: We present the NeuroPoint Alliance and Institute for Healthcare Improvement (NPA-IHI) program as a novel attempt to develop quality improvement (QI) tools designed from registry data that improve the quality of care delivered. To date, no group has combined insights from a national registry with validated QI methods. Reducing readmission and length of stay (LOS) after elective lumbar fusion was chosen as the pilot module (Fig1).

Methods: The NPA-IHI program prospectively enrolled patients undergoing 1-3 level lumbar fusion across 8 institutions. 1) Research Phase: Literature review and stakeholder interviews were completed, followed by Quality Outcomes Database (QOD) analysis to identify key drivers of readmission and LOS. 2) Intervention Phase: Specific interventions were designed to address the previously identified drivers. 3) Measurement Phase: The application of these interventions and overall patient experience were measured.

Results: A total of 232 patients were included with an average LOS of $3.4 \pm 1.8$ days for one-level fusion $(\mathrm{N}=143)$ and $4.4 \pm 2.4$ days for two-level fusion (N=89)(Fig2-3). 1) Research Phase: Pain control and medical comorbidities emerged as key drivers of both outcomes. Eighty percent of readmissions occurred within the first 4 weeks and $46 \%$ within 14 days. 2) Intervention Phase: The "Rounding Tool" checklist was developed, comprised of 9 care parameters and utilized daily at bedside (Fig3). 3) Measurement Phase: Successful application of the "Rounding Tool" was seen. The top three reasons preventing discharge were mobility status
(64\%), indwelling devices (51\%), and pain control (38\%)(Table1). Preliminary data shows the majority of patients reported a positive experience (Fig4).

Conclusion: We present a novel method for combining insights gained from a national registry with continuous quality improvement protocols in a multi-institutional setting. Future efforts will be focused on preoperative, hospital, and postdischarge interventions (Figs5-7) and using the registry platform itself as a method of gathering process data at the point of service.

320 The Effect of Preoperative Opioid Use on Single Level Interbody Fusion for Degenerative Lumbar Spondylolisthesis.

Alp Ozpinar, MD; Nima Alan, MD; David O. Okonkwo, MD, PhD; Adam S. Kanter, MD; D. Kojo Hamilton, MD

Introduction: The use of prescription opioids may predispose patients to chronic opioid use .We hypothesized that the use of preoperative narcotics may lead to poorer patient outcomes in patients with degenerative lumbar spondylolisthesis undergoing single level interbody fusion.

Methods: A consecutive series of patients undergoing L4-5 transforaminal lumbar interbody fusion (TLIF) for degenerative lumbar spondylolisthesis were retrospectively analyzed. Patients were categorized according to the presence or absence of prescribed preoperative opioid use $>6$ months using standard oral morphine dose-equivalents. Outcomes included the Oswestry Disability Index (ODI), Visual Analogue Scale (VAS) and Short Form 36 Physical and Mental Summary Scores (SF36-PCS, SF36-MCS). Between-group comparisons were performed using analysis of variance.

Results: 63 patients ( $60 \%$ female) were identified with mean age of 56.4 \pm 12.3 -years. Mean follow-up 13.6 months. 31 (48.9\%) patients were prescribed preoperative opioids, with a mean oral morphine equivalent of $17.6 \pm 15.3 \mathrm{mg} / \mathrm{day}$. Mean hospital length of stay (LOS) was 2.7 \pm 2.1 -days for the non-opioid group and 3.8 \pm 2.8 days for the opioid group. Mean preoperative ODI was $49.9 \pm 14.3$ for non-opioid versus $44.5 \pm 13.1$ for opioid. Postoperatively, ODI was $25.9 \pm 22.8$ and $28.2 \pm 18.9$ for the two groups respectively. Mean preoperative VAS for the two groups was nearly identical (7.18 \pm 1.8 , $7.19 \pm 2.0$ ), while mean postoperative VAS was $2.7 \pm 2.1$ and $3.8 \pm 2.8$ respectively. A trend was observed for the change in postoperative from preoperative ODI and VAS, with the non-opioid group showing a greater magnitude of improvement (-24.0 vs. -16.2-points), (-4.45 vs. -3.38$)$, respectively. The mean preoperative SF36-PCS (32.3 \pm 8.3$)$ preoperative SF36-MCS $(40.7 \pm 13.3)$, postoperative SF36-PCS (42.5 \pm 10.6$)$ and postoperative SF36-MCS (47.1 \pm 11.8$)$ did not differ by opioid use group.

Conclusion: In a preliminary cohort of consecutive patients undergoing single level interbody fusion for degenerative lumbar spondylolisthesis, trends exist between preoperative opioid use, HLOS, and postoperative improvement in ODI and VAS. The convergence of preoperative opioid usage and pain control warrants future prospective studies of larger sample sizes.

321 Preoperative Hemoglobin Level is Independently Associated with Increased Healthcare Utilization After Elective Spinal Fusion (e"3 Levels) in Elderly Male Spine Deformity Patients

Aladine A. Elsamadicy, BE; Owoicho Adogwa, M.D., M.P.H; Michael Ongele, B.S.; Amanda Sergesketter; Aaron Tarnasky, B.S.; Joseph S. Cheng, MD, MS; Carlos Antonio Bagley, MD; Isaac O. Karikari, MD

Introduction: Recently, hospitals and health systems are 
investing considerable resources into the pre-operative identification of at-risk patients. The aim of this study is to determine if a low pre-operative hemoglobin for adult males is risk factor increased healthcare resource utilization.

Methods: The medical records of 204 elderly ( $=60$ years old) deformity patients undergoing elective spinal fusion at a major academic institution from 2005 to 2015 were reviewed. Low Hemoglobin was designated as a hemoglobin level $<13.5 \mathrm{~g} /$ dl. We identified $83(40.7 \%)$ male-patients with a pre-operative low-hemoglobin and 121 (59.3\%) with normal levels (Low: $n=83$; Normal: $\mathrm{n}=121$ ). Patient demographics, comorbidities, and 30-day post-operative complication and readmission rates were collected for each patient. The primary outcome investigated in this study was complication rates and length of hospital stay.

Results: 204 adult male patients (Low Hgb: $n=83$; Normal Hgb: $n=121$ ) were included in this study. There were significant differences in the hemoglobin (Low Hgb: $12.3 \pm 0.9 \mathrm{~g} / \mathrm{dL}$ vs. Normal Hgb: $14.9 \pm 1.0 \mathrm{~g} / \mathrm{dL}, \mathrm{p}<0.0001$ ) and hematocrit (Low Hgb: $0.372 \pm 0.029$ vs. Normal Hgb: $0.439 \pm 0.028, \mathrm{p}<0.0001)$. There were no significant differences in intra-operative variables and complication rates. There was a significant difference in the incidence of postoperative delirium with the low hemoglobin group have a three-fold increase in comparison to the normal hemoglobin group, (Low Hgb: $21.7 \%$ vs. Normal Hgb: $5.8 \%, \mathrm{p}=0.0007$ ). There was a significant difference in the length of stay in the hospital between both groups, with the low hemoglobin group having an increased hospital stay when compared to the normal hemoglobin cohort (Low Hgb: 8.1 \pm 5.9 days vs. Normal Hgb: $4.8 \pm 2.5$ days, $\mathrm{p}<0.0001)$.

Conclusion: Our study demonstrates demonstrate that male adult patients with a low hemoglobin (hemoglobin level $<13.5 \mathrm{~g} /$ dl.) have an increased length of stay in the hospital and higher rates of delirium post-operatively.

322 Correlation Between Anterior Lumbar Interbody Fusion Cage Height and Foraminal Height and Area: a CT Analysis

Kai-Yuan Chen, MD; Dean Chou, MD; Rong-Ping, MD Zhou; Can Zhang

Introduction: Anterior lumbar interbody fusion (ALIF) affords indirect foraminal decompression and induction of lumbar lordosis. We use computed tomography (CT) to measure disc height, foraminal height $(\mathrm{FH})$ and foraminal area (FA) to correlate cage height with pre- and post-operative DH, FA and FH.

Methods: foraminal area (FA) to correlate cage height with pre- and post-operative DH, FA and FH. Methods Retrospective review of consecutive patients who undergoing ALIF from 2007 to 2016. Only patients who had pre- and post-operative CT scans were included. DH, FA, and FH were measured bilaterally by CT before and after surgery. The cage height at each level was obtained from implant records.

Results: 24 patients and 42 levels were analyzed. Improvement of DH, FH and FA after ALIF was found. The overall improvement in FH, FA and DH was $15 \%, 25 \% 43 \%$, respectively. Subgroup analysis of cage heights and FH, FA and DH, respectively, were the following: $11 \mathrm{~mm}$ improved $16 \%, 5 \%$ and $19 \% ; 12 \mathrm{~mm}$ improved $11 \%, 35 \%$, and $139 \%$. 13mm improved $15 \%, 27 \%$, and $34 \% .14 \mathrm{~mm}$ improved $32 \%, 51 \%$, and $74 \%$. $15 \mathrm{~mm}$ improved $13 \%$, $24 \%$, and $62 \%$. $17 \mathrm{~mm}$ improved $24 \%, 73 \%$, and $113 \%$.

Conclusion: ALIF shows improvement in FH, FA and DH after surgery; however, cage height is not linearly correlated with the percentage of improvement in FH, FA and DH
323 Inpatient Versus Outpatient Venous Thromboembolic Events After Spine Surgery: An Analysis of 6869 Consecutive Patients Revealing Distinct Risk Factors

Michael Cloney, MD; Connor Driscoll, BS; Jonathan Tad Yamaguchi, BS; Benjamin Hopkins, BS; Tyler R. Koski, MD; Zachary Adam Smith, MD; Nader S. Dahdaleh, MD

Introduction: Venous thromboembolic events (VTEs) are a common cause of readmission and morbidity for patients undergoing spine surgery. The existing literature has not examined risk factors for VTEs in the outpatient setting distinct from VTEs in the inpatient setting.

Methods: We retrospectively collected data on all patients who underwent spine surgery at our institution between 2009 and 2015. Multivariable analysis was performed to identify demographic, clinical, and procedural factors independently associated with VTE events during three time periods: during the initial inpatient admission, after discharge following the index surgery and at any point during the first 30 postoperative days.

Results: Having an outpatient DVT was independently associated with readmission within 30 days (OR 62.619, $\mathrm{p}=0.002$ ). Time to onset of VTE events was significantly longer for patients with an osteotomy than for patients with a fracture $(7.43$ v. 4.28 days, $\mathrm{p}=0.0180$ ). Multiple factors were associated with DVT or $\mathrm{PE}$ in both the inpatient and outpatient settings. Factors associated with PE as an inpatient only include surgery $>4$ hours (OR 30.820, $\mathrm{p}<0.001$ ), fracture (OR 6.913, $\mathrm{p}=0.004$ ), preoperative IVC filter placement (OR 3.135, $\mathrm{p}=0.029)$, and prophylactic anticoagulation (OR $0.285, p=0.017)$. Factors associated with $\mathrm{PE}$ as an outpatient only include corpectomy (OR 541.271, p=0.009), EBL >500 (OR $2467.798, \mathrm{p}=0.002)$. Factors associated with DVT as an inpatient only include preoperative IVC filter placement (OR 6.380, $\mathrm{p}=0.000)$, longer length of stay $(\mathrm{OR} 1.083, \mathrm{p}=0.000)$, and a prior history of DVT (OR 3.639, $\mathrm{p}<0.001)$. Factors associated with DVT as an outpatient only include having a history of PE (OR $45.142, \mathrm{p}=0.000)$, having a corpectomy (OR 26.670, $\mathrm{p}=0.002)$, and having an osteotomy (OR 18.877, $\mathrm{p}=0.041$ )

Conclusion: DVT as an outpatient is associated with 30-day readmission. The factors associated with inpatient VTE events are distinct from the factors associated with outpatient VTE events. Time-to-VTE event varies by pathology and procedure.

324 Computer-assisted Tracking Minimizes Radiation Exposure While Maintaining Localization and Image Guidance for Instrumentation - A Randomized Trial

John Berry-Candelario, MD; Farah Hamouda; Juan S. Uribe, MD, FAANS; Chester Yarbrough, MD, MPHS

Introduction: Surgical instruments must be repositioned multiple times throughout an intervention and often adjusted repeatedly until the physician is satisfied. The sheer number of x-ray images needed to position the instrument to the desired location can be staggering. Using a novel computer assisted technology, TrackX, a computer shows the movement of the instrument as an instrument is adjusted, mimicking live fluoroscopy. This randomized controlled study was performed to determine if TrackX can allow instruments to be localized during spine procedures with less x-rays, greater accuracy, and in less time than unassisted fluoroscopy.

Methods: A cadaveric study was undertaken. Multiple physicians were asked to identify the ideal location for a surgical procedure and then place an instrument to this location both with and without computer assistance, randomly assigned to reduce the 
impact of learning. Number of xrays, radiation exposure and time to perform each procedure was recorded.

Results: Five physicians participated in the study, performing several of nine different surgical procedures. Twenty-three trials were completed both with and without the assistance of TrackX, ranging from percutaneous pedicle screws to XLIF. The total time to localize for all 23 cases was $45.4 \%$ more when performed without assistance. The total number of xrays taken was $74.8 \%$ less. The total radiation exposure was $91.8 \%$ less. Significant radiation reduction was experienced for every procedure. Physicians were able to place the instrument with TrackX on the first attempt in $82.6 \%$ of trials and by the second attempt in $100 \%$ of trials. Without, the physicians were never able on the first attempt and took 4.65 (2-8) images on average.

Conclusion: Computer assisted instrument tracking is able to dramatically reduce the number of unnecessary images taken when performing a fluoroscopic procedure. Overall, this resulted in $91.8 \%$ radiation reduction and a significant time savings.

325 Postoperative Complications Following Anterior Lumbar Interbody Fusion in 1021 Patients

Joseph A Osorio, MD, PhD; Winward Choy; Sivan Marcus; Lillian Lai; Dominic Amara, BA; Alexander Tenorio; Rishi Kumar Wadhwa, MD; Serena Hu; Bobby Tay, MD; Shane Burch; Sigurd Berven, MD; Vedat Deviren, MD; Sanjay S. Dhall, MD; Aaron John Clark, MD, PhD; Dean Chou

Introduction: Anterior lumbar interbody fusion (ALIF) carries a unique complication profile and associated risk factors. We report our institutional experience to elaborate on perioperative outcomes.

Methods: A retrospective chart review of patients receiving an ALIF from April 2007 to November 2016 at large tertiary academic center was performed. Indications, perioperative data, complications, and outcome data were aggregated and analyzed. T-test and ANOVA was used to compare differences in means.

Results: A total of 1021 patients undergoing ALIF was included. Mean age was 57.7, with $45 \%$ males. Common indications include degenerative disc disease $(29.1 \%)$, scoliosis $(16.2 \% \%)$, spondylolisthesis $(13.4 \%)$, pseudoarthrosis $(12.1 \%)$, and spinal stenosis $(6.6 \%)$. Single and two level ALIF were performed in $36.8 \%$ and $41.2 \%$ of cases, respectively. Mean estimated blood loss (EBL) was $386 \mathrm{ml}$, correlating with number of levels fused. EBL for 1, 2, 3, 4 level fusion was 220, 389, 602, and $1317 \mathrm{ml}$, respectively $(\mathrm{p}=0.007)$. EBL $>500 \mathrm{ml}$ was present in 81 cases, of which 38 required transfusion. Mean procedure duration was 252 minutes, also correlating with number of levels fused. Duration was 229, 252, 299, and 301 minutes for 1, 2, 3, and 4 level ALIF, respectively $(\mathrm{p}=0.019)$. Following ALIF, second staged procedure was performed in $77.0 \%$ of patients, predominantly posterior fusion. The most common complications comprised venous injury $(12.6 \%)$, ileus $(6.5 \%)$, urological complications $(3.2 \%)$, ventral hernia $(3.1 \%)$, PE/DVT $(1.7 \%)$, anterior seroma $(0.6 \%)$, and peripheral nerve damage $(0.3 \%)$. There was 1 arterial injury and 1 death following massive PE.

Conclusion: In the largest case series to date, ALIF is a safe and effective approach for lumbar fixation. Number of levels fused correlated with procedure time and EBL. Complication profile included venous injury, ileus, urological complications, ventral hernia, and thromboembolism. Arterial injury only occurred in 1 case, and rates of significant venous injury requiring transfusion is low.
326 Early Ambulation Leads to Decreased Length of Hospital Stays in Patients Undergoing Lumbar Laminectomy

Jang Yoon, MD, MS; James Tabe Ebot, BS, MS; Gaetano De Biase, MD; Selby G. Chen, MD; Stephen M. Pirris, MD; Eric W. Nottmeier, MD; Clarence B. Watridge, MD; Hugh Gordon Deen, MD; Ronald Reimer, MD; Mark A. Pichelmann, MD; Robert E. Wharen, MD

Introduction: Physicians are under increasing pressure to shorten inpatient hospital stays without compromising patient care. Lumbar laminectomy is a commonly performed procedure, resulting in a large number of inpatient days at Mayo Clinic Florida. After implementation of a clinical protocol that encouraged early post-operative ambulation, we prospectively compared patients who were mobilized early vs. historical cohort.

Methods: Early ambulation protocol was initiated in October 2012 , in which all patients undergoing lumbar laminectomy to be mobilize within 1 hour of admission to neurosurgical floor. We excluded those who underwent instrumented fusion in addition to decompression; those with surgical complications such as durotomy, those with post-operative medical complications that prolonged hospital stays independent of surgical outcomes.

Results: We compared early ambulation group that included those who underwent lumbar laminectomy from 2013 to 2015 $(n=635)$ to those who had lumbar laminectomy from 2009 to $2011(n=402)$ at Mayo Clinic Florida. The mean length of stay decreased from 2.6 to 2.12 days ( $p=0.0014$ ). There were no differences between the groups in terms of age, gender, number of laminectomy levels, prior surgery, or techniques used (MIS vs. open), although the average operating time was 36 minutes shorter in the early ambulation group (182 vs. $176 \mathrm{~min}$ : $\mathrm{p}=0.1506)$.

Conclusion: Early ambulation following laminectomy is safe and leads to improved postoperative recovery and earlier hospital discharge.

327 Characterization of Neurologic Injury Using the Area Under the Curve of Somatosensory Evoked Potentials

Ahmed Jorge; James Zhou, MD; Parthasarathy D Thirumala; Shreya Umredkar; Rohan Krishnan

Introduction: Intra-operative neurophysiological monitoring (IONM) with somatosensory evoked potentials (SSEP) has been shown to reduce the risk of motor deficits due to SCI during corrective surgery. Nonetheless, peaks are oftentimes difficult to distinguish, as they contain noise and oftentimes signals broaden specially after injury or anesthesia. Here, we present a novel model that assesses SSEP signals in a single number by summing the area under the SSEP curve.

Methods: 26 Adult Sprague-Dawley rats were randomly divided into four experimental groups, which received contusive spinal cord injures (SCI) of various degrees (ranging from 100 to 200 kilodynes) after a laminectomy was performed. The control group underwent laminectomy only. Neurophysiological testing was performed on day 1 , day 3 , and day 7 after surgery. The SSEP values obtained were processed and the absolute area under the curve was calculated.

Results: The mean values for the AUC for the experimental groups are significantly different from the control group across three days post operative $(\mathrm{p}<0.0001$, for all days). Moreover, analysis of variance reveals that the only significant parameter that accounts for the variability in AUC is impact strength, $\mathrm{p}<00001$ (number of days and subject parameters were not significant).

Conclusion: This investigation proposes a new method to observe and analyze an SSEP signal after an iatrogenic spinal cord 
injury has occurred. The AUC was successful at distinguishing significant differences between subjects in the control group versus in the impact group by simply accounting for one number per signal, i.e. the AUC. In this fashion, simplifying IONM with less parameters can help clinicians effectively and optimally gauge a patient's SCI status.

328 External Validation of the SORG Nomogram and Classic Scoring Algorithm

A. Karim Ahmed; C. Rory Goodwin, MD, PhD; Amir Heravi; Rachel C. Kim, BS; Nancy A Abu-Bonsrah, MD; Eric W. Sankey, MD; Rafael De la Garza-Ramos, BA; Joseph H. Schwab; Daniel M. Sciubba, MD

Introduction: Skeletal Oncology Research Group (SORG) scoring systems were developed to address drawbacks of the previous prognostic calculators. The current study aimed at assessing the accuracy of the SORG Classic Scoring and SORG Nomogram.

Methods: Patients who underwent surgical resection for metastatic spine disease at a single institution were included. A pre-operative score for all 176 patients was calculated utilizing the SORG Classic Scoring algorithm and SORG Nomogram. Receiver operating characteristic analysis (ROC) modeling was utilized to produce area under the curve (AUC) for the accuracy of each test. The predictive survival accuracy of each scoring system was compared for all patients, and by subgroups of specific primary tumor etiology.

Results: The median survival for the Good Prognosis, Intermediate Prognosis, and Poor Prognosis group was 880 days (95\% CI 516-1227), 352 days (95\% CI 238-484), and 163 days (95\% CI 111-207) from the time of surgery, respectively. A logrank test revealed statistical significance $(\mathrm{p}<0.015)$ between each defined prognostic group based on the preoperative SORG Classic Scoring algorithm. ROC curves demonstrated sufficient accuracy (AUC $>0.7$ ) at 30 days, 90 days, and 365 days following surgery.

Conclusion: This study successfully validated the SORG Classic Scoring Algorithm and SORG Nomogram, in an independent patient cohort from a single institution and identified factors strongly associated with survival for patients undergoing surgery for metastatic spine disease.

329 Biomechanical Evaluation of Posterior Cervical Cages as a Supplement to the Anterior Integrated Cage and Comparison with Plated ACDF

Leonard I. Voronov, MD, PhD; Robert M. Havey; Saeed Khayatzadeh, PhD; Gerard Carandang, MS; Avinash G Patwardhan, $\mathrm{PhD}$

Introduction: Anterior integrated cage (AIC) constructs provide an alternative technique to ACDF for treatment of adjacentlevel disease. By placing the AIC adjacent to a previous platedfusion, the need for an extensive soft-tissue exposure and hardware removal is obviated. Posterior Cervical Cages offer the potential for increased stabilization with minimal tissue disruption and may compliment AIC constructs. Purpose: to compare the effectiveness of zero-profile anterior integrated cage with and without posterior instrumentation and to compare this construct with plated-ACDF without posterior instrumentation.

Methods: Fourteen specimens $(42.4 \pm 6.8$ years $)$ were divided in two groups ( $\mathrm{n}=7 /$ group) and tested in flexion-extension (FE), lateral bending (LB), and axial rotation (AR) to $\pm 1.5 \mathrm{Nm}$. Experimental protocol: Group 1: Intact, C6-C7 AIC, Supplemental C6-C7 bilateral PC, C3-C5 AIC, Supplemental C3-C5 bilateral
PC. Group 2: Intact, C6-C7 plated-ACDF, C3-C5 plated-ACDF.

Results: Group I: C6-C7 AIC reduced ROM from 9.3 $\pm 2.0 \mathrm{deg}$ to $5.9 \pm 2.9 \mathrm{deg}$ in $\mathrm{FE}$, from $7.3 \pm 1.6 \mathrm{deg}$ to $1.8 \pm 1.3 \mathrm{deg}$ in $\mathrm{LB}$, from $5.8 \pm 2.0 \mathrm{deg}$ to $2.7 \pm 2.0 \mathrm{deg}$ in AR. Supplemental PC reduced ROM: $1.0 \pm 0.5 \mathrm{deg}$ in flexion-extension, $0.3 \pm 0.1 \mathrm{deg}$ in $\mathrm{LB}, 0.5 \pm 0.2 \mathrm{deg}$ in AR. The results were similar under two-level fusion (C3-C4/ C4-C5 AIC), with significant reductions in ROM, and then further ROM reduction with addition of PC. Group I vs. II: The $\mathrm{AIC}+\mathrm{PC}$ construct was more rigid than the plated-ACDF. At C6-C7, AIC+PC reduced flexion-extension ROM to $1.0 \pm 0.5 \mathrm{deg}$, compared to $2.5 \pm 0.8 \mathrm{deg}$ after plated-ACDF. In $\mathrm{LB}, \mathrm{AIC}+\mathrm{PC}$ reduced $\mathrm{C} 6-\mathrm{C} 7 \mathrm{ROM}$ to $0.3 \pm 0.1 \mathrm{deg}$, compared to $1.6 \pm 0.7 \mathrm{deg}$. In $\mathrm{AR}, \mathrm{AIC}+\mathrm{PC}$ reduced $\mathrm{C} 6-\mathrm{C} 7 \mathrm{ROM}$ to $0.5 \pm 0.2 \mathrm{deg}$, compared to $1.7 \pm 0.4 \mathrm{deg}$. At the upper cervical levels, AIC+PC construct performed similarly or better than plated-ACDF in limiting motion.

Conclusion: Supplementation of single- and multilevel AIC using bilateral PC provided a significant stability increase. The biomechanical effectiveness of PC supplementing AIC implantation is comparable to plated-ACDF for single- and two-level fusion constructs. There may be a clinical benefit to using this implant in revision cases.

330 Impact of Expandable Vs. Static Interbody Cage Devices on Cervical Sagittal Alignment After Corpectomy

Brandon W Smith, MD, MSCR; Jacob R. Joseph, MD; Michael Kirsch; Yamaan S. Saadeh, MD; Paul Park, MD

Introduction: Cervical corpectomy is a well-established option for the treatment of symptomatic cervical spondylosis. Recently, the expandable interbody cage has been popularized in anterior reconstruction. The impact of expandable cage technology on sagittal alignment is unknown.

Methods: Patients undergoing cervical corpectomy utilizing an expandable interbody cage at a single institution were identified. Eligible controls were identified as patients undergoing utilizing a static cage with pre-operative and post-operative lateral cervical radiographs. Patient data including indication, ASA, BMI, smoking status, osteoporosis, levels, additional procedures, and imaging were extracted. Preoperative and postoperative x-rays of the cervical spine were evaluated for segmental and overall cervical lordosis, as well as the C2-C7 sagittal vertebral axis (cSVA).

Results: A series of 41 patients undergoing cervical corpectomy with expandable cages and 17 controls undergoing cervical corpectomy with static cages were identified. Overall 30(51.7\%) were male and $28(48.3 \%)$ were female. The average age was $57.3 y r s(19 y r s-85 y r s)$ at the time of the operation. Of the 41 eligible patients with expandable cages, $30(73 \%)$ had preoperative and postoperative lateral cervical spine radiographs. The mean overall cervical lordosis pre-operatively was 0.92 degrees in the expandable group and 2.34 degrees in the static group. On immediate post-operative imaging there was a significant increase in lordosis of 8.6 degrees $(\mathrm{P}<0.0001)$ in the expandable group and a non-significant increase 2.3 degrees $(\mathrm{P}=0.17)$. Similarly, the mean segmental lordosis significantly increased after surgery by 5.3 degrees $(\mathrm{P}<0.0001)$ in expandable cages, while there was a non-significant increase of 1.6 degrees $(\mathrm{P}=0.23)$ in the static cage group. There was a small, but statistically significant decrease in cSVA of $0.67 \mathrm{~cm}(\mathrm{P}=0.004)$ in the expandable group, but a slight non-significant increase of $0.16 \mathrm{~cm}(\mathrm{P}=0.69)$ in the static cage group.

Conclusion: Segmental and overall cervical lordosis are significantly increased with use of an expandable cage, however, these parameters did not significantly increase when a static cage was utilized. 
331 Incidence and Risk Factors for Superior Facet Joint Violation in Lumbar Fusion

Alisson R Teles, MD; Michael Paci, MD; Gabriel Gutman; Fahad H Abduljabbar, MBBS; Jean Ouellet, MD, FRCSC1; Michael Weber, MD, PhD, FRCSC, 1; Jeff D. Golan, MD

Introduction: Cranial facet joint violation has been an underappreciated consequence of pedicle screw placement in the lumbar spine. The objetive of this study was to evaluate the anatomical and surgical risk factors for screw-related facet joint violation at the superior level in lumbar fusion.

Methods: Retrospective review of a consecutive series of posterior lumbar instrumented fusions performed by a single surgeon. Inclusion criteria was primary lumbar fusion of 1 or 2 levels for degenerative disorders. The following variables were analyzed as possible risk factors: surgical technique (percutaneous versus open screw placement), depth of surgical field, degree of anterior slippage of the superior level, pedicle and facet angle, and facet degeneration of the superior level. The postoperative CT was evaluated by two independent reviewers. Axial, sagittal, and coronal views were reviewed and pedicle screws were graded as intra-articular if they clearly interposed between the superior and inferior facet joints of the superior level. Multivariate logistic regression analyses were conducted to assess the factors associated with this complication.

Results: 131 patients were included. Inter-observer reliability for facet joint violation assessment was high (Kappa $=0.789)$. The incidence of superior facet joint violation was $12.59 \%$ per top level screw $(\mathrm{N}=33 / 262)$. The rate of facet violation was $28.0 \%$ in percutaneous technique group $(\mathrm{n}=14 / 50)$ and $12.3 \%$ in open group $(\mathrm{n}$ = 10/81), OR: 2.26; CI95\%: 1.09 4.21; P = 0.024. In multivariate logistic regression analysis, independent predictors of facet violation were percutaneous screw placement (Adjusted OR: 3.31; CI95\%: 1.42 7.73; $\mathrm{P}=0.006$ ), right side pedicle screw (Adjusted OR: 3.14; CI95\%: 1.29 7.63; $\mathrm{P}=0.011)$, and facet angle greater than 45 (Adjusted OR:10.95; CI95\%: 4.64 25.84; $\mathrm{P}<0.0001$ ).

Conclusion: The incidence of facet joint violation is higher in percutaneous minimally invasive than in open technique for posterior lumbar spine surgery. Also, coronal orientation of the facet joint is a significant risk factor independently of the surgical technique.

332 The Five-repetition Sit-to-stand Test (5R-STS): A Novel Tool for the Objective Assessment of Degenerative Spinal Disease

\section{Victor E. Staartjes, BS; Femke Beusekamp; Marc 1 Schroder}

Introduction: Currently, clinical determination of the burden of disease of lumbar degenerative diseases occurs by use of validated questionnaires that describe the patients' subjective pattern of complaints. However, the additional objective testing of functional status may become of prime importance. The fiverepetition sit-to-stand (5R-STS) test is a quick, simple, objective test validated for use in pulmonary disease. We aim to assess the effectiveness of the 5R-STS as an objective measure of functional impairment in spinal degenerative disease.

Methods: In a prospective study, patients with degenerative diseases of the lumbosacral spine and healthy volunteers performed the 5R-STS. On a standardized chair, patients are asked to fold their hands across their chest, to stand up from a sitting position and sit down again once for practice. Then, patients are asked to repeat the maneuver 5 times as fast as possible. The time in seconds is the result of the test. Outcomes were correlated with visual analogue scales (VAS) for back and leg pain, EQ-5D,
Oswestry Disability Index (ODI), and Roland-Morris Disability Questionnaire (RMDBQ).

Results: 75 patients and 15 healthy volunteers were recruited. Most patients (83\%) presented with lumbar disc herniation. Mean age was $54 \pm 13$ years, and mean BMI was $25.3 \pm 3$.4. One patient was unable to complete the test. Patients had significantly higher 5R-STS scores than healthy controls $(11.8 \pm 4.1$ versus $7.2 \pm 2.4$ seconds, $p=0.001)$. 5R-STS scores were strongly correlated with validated questionnaires (all $\mathrm{p}<0.001$ ). This strong correlation persisted when controlling for age and BMI in a regression model (all $\mathrm{p}<0.05$ ).

Conclusion: The 5R-STS is a fast, easy, safe and effective means for the objective assessment of functional impairment in patients with degenerative diseases of the lumbar spine. Further prospective research in larger cohorts, as well as validation of testretest reliability and interrater agreement are indicated.

333 Systematic Review of Neurological Complications Following Surgical Treatment of Lumbar Spondylolisthesis in Adults

Noojan Kazemi, MD; Jerry Lee Walters; Arunprasad Gunasekaran; Paul Lee, MD; Taylor Anne Wilson

Introduction: While there are several studies examining total complication rates following surgical treatment for lumbar spondylolisthesis, the data specifically addressing neurologic complications remains limited. The aim of this study was to perform a systemic review of all current studies that assessed neurological complications in treatment of spondylolisthesis and to identify associated predictive factors.

Methods: A comprehensive review of the all publications mentioning surgical treatment, neurological complications, and lumbar spondylolisthesis between 2007 and 2017 inclusive from electronic databases was performed. The following variables were determined: neurological complication rate, age, grade and percentage of spondylolisthesis reduction, surgical approach, transient versus permanent neurological deficit, mean blood loss, and mean operative time.

Results: A total of 23 studies meeting the inclusion criteria were identified. Of these, 14 studies met a priori inclusion criteria. Of a total of 78,598 patients, $767(0.98 \%)$ experienced neurological deficits post operatively including acute, delayed, transient, or permanent new sensory or motor deficits. Mean age was 62 years with mean operative time of 185 minutes and mean blood loss of $480 \mathrm{~mL}$ per level. The most prevalent surgical technique involved a posterior lumbar interbody fusion (PLIF) with or without reduction, compared with decompression or postero-lateral arthrodesis. The majority of deficits $(75.4 \%)$ were transient and involved new sensory deficits. The majority of these resolved in the early postoperative period (range 3-25 weeks). The average reduction of spondylolisthesis achieved was $14 \%$. When weighted for sample size, there was significant correlation between complication rate and mean age, blood loss and operative time, but not degree of slip reduction.

Conclusion: There is a paucity of data specifically examining neurological deficits following surgical treatment for lumbar spondylolisthesis. In this systematic review, we demonstrate statistically significant correlation between neurological deficits and operative risk factors including age, blood loss and operative time but importantly not spondylolisthesis reduction. 
334 Anterior Versus Posterior Approaches for Thoracic Disc Herniation: Association with Postoperative Complications

Mohamed Elminawy, MBBCh; Panagiotis Kerezoudis; Kenan Rajjoub, BA; Mohammed Ali Alvi, MD; Sandy Goncalves; Abdullah Alamoudi, MBBS; Mohammed Adeeb Sebai; Anshit Goyal, MBBS; Elizabeth B Habermann, PhD; Mohamad Bydon, MD

Introduction: Thoracic disc herniation (TDH) represent 1.5 to $4 \%$ of all intervertebral disc herniations. Surgical treatment can be divided into anterior, lateral and posterior approaches and is an area of contention in the literature. Available evidence consists mostly of single-arm, single-institutional studies with limited sample sizes. We sought to to retrospectively investigate 30-day surgical outcomes following excision of TDH utilizing a national surgical registry.

Methods: The American College of Surgeons - National Surgical Quality Improvement Program (ACS-NSQIP) was queried for cases that underwent anterior (thoracotomy or thoracoscopy), lateral (extracavitary or costotransversectomy) or posterior (transpedicular or laminectomy) surgery for a primary diagnosis of TDH between 2012 and 2015.

Results: A total of 388 patients ( $48 \%$ females) were included in the analysis. An anterior approach was used in 65 patients, lateral approach in 34, transpedicular approach in 90 and laminectomy in 199. Overall, baseline demographics and clinical characteristics were similarly distributed between the four procedure groups. Patients undergoing an anterior approach spent, on average, 2-3 more days in the hospital compared to all other groups $(\mathrm{p}<.001)$. Furthermore, the anterior approach group was more likely to have developed a major complication (27\%) compared to the lateral $(8 \%)$, transpedicular $(18 \%)$ or laminectomy groups $(14 \%)(\mathrm{p}=0.13)$. Unplanned 30-day readmission and return to the operating room occurred in $5-8 \%$ of all patients $(\mathrm{p}=0.69$ and 0.63 , respectively). Lastly, the majority of the patients were discharged to home or a home facility (anterior-74\%; lateral-81\%; transpedicular-68\% and laminectomy-74\%, $\mathrm{p}=0.58$ ).

Conclusion: Anterior approaches had longer length of stay and higher, although not statistically significant, complication rates. No difference was found with regard to discharge disposition. In light of these findings, surgeons should weigh the risks and benefits of each surgical technique during tailoring of decision making.

335 Comparing Outcomes of Fusion Versus Discectomy Alone for One-time Recurrent Lumbar Disc Herniation: A Systematic Review and Meta-analysis

Panagiotis Kerezoudis; Sandy Goncalves; Joseph Cesare; Dennis Kurian, B.Sc; Mohammed Ali Alvi, MD; Yagiz Ugur Yolcu; Mohamed Elminawy, MBBCh; Anshit Goyal, MBBS; Mohamad Bydon, MD

Introduction: Recurrent lumbar disc herniation (RLDH) occurs in approximately $25 \%$ of patients undergoing primary discectomy for lumbar disc herniation. Current surgical treatment options include repeat discectomy or discectomy supplemented with fusion. Significant contention exists within the surgical spine community with regard to the most effective treatment modality. Herein, we sought to compare postoperative surgical and patient reported outcomes following fusion versus discectomy alone for RLDH

Methods: The electronic literature search was performed in Ovid Medline/Pubmed, EMBASE and Cochrane, Scopus and China National Knowledge Infrastructure for human studies directly comparing repeat discectomy with fusion for ipsilateral or contralateral RLDH. Random effects meta-analysis was conducted to pool the estimates of effect, using mean differences (MD) and odds ratio(OR) for continuous and categorical outcomes, respectively.

Results: A total of 1405 patients (746 fusions and 659 discectomies alone) from 15 studies (13 observational and 2 randomized controlled trials were analyzed. Mean time to reherniation was $54.4 \pm 30.4$ months, while average follow-up time was $40 \pm 11.7$ months (range: 12-92.6). Fusions were associated with significantly longer operative time (MD: 67 minutes, 95\% CI: 50 to 85), higher estimated blood loss(MD: $222 \mathrm{ml}$; 95\% CI: 164 to 280) as well as longer hospitalizations(MD: 2.7 days, $95 \%$ CI: 1.6 to 3.8) compared to discectomies. Changes in PRO scores from baseline to last follow-up were similar between the two groups, including VAS-back pain (MD, -0.3 ; 95\% CI, -1.4 to 0.7 ), VAS-leg pain (MD, $-0.3 ; 95 \% \mathrm{CI},-1.4$ to 0.7 ), ODI (MD, 0.6 ; $95 \% \mathrm{CI},-0.2$ to 1.4), JOA (MD: $1.0 ; 95 \%$ CI: 0.02 to 2.0 ) and McNab satisfaction (OR: $1.5 ; 95 \%$ CI, 0.9 to 2.3 ).

Conclusion: Available evidence shows that fusion is associated with longer hospital stays, operative times and higher intraoperative blood loss. Future longitudinal, randomized controlled trials should be completed to validate any associations found in this study.

336 The Association of Post-Operative Opioids and Pain Management Scores on the HCAHPS Survey in Patients Following Lumbar Spine Surgery

Robert Winkelman, BS; Jay Levin; Gabriel Alexander Smith, MD; Joseph E Tanenbaum, BA; Thomas E. Mroz, MD; Michael P. Steinmetz, MD

Introduction: The recent use of patient experience scores as quality metric in healthcare has generated considerable concern amongst healthcare providers. One major concerns is the Hospital Consumer Assessment of Healthcare Providers and Systems (HCAHPS) survey may be incentivizing providers to prescribe opioids more liberally to patient in an effort to improve these scores. Since there are presently no reports in the medical literature linking prescription opioids to HCAHPS scores, the goal of our study was to investigate the relationship of post-operative opioids and HCAHPS pain management scores in lumbar spine surgery patients.

Methods: The present study is a retrospective cohort analysis of 210 patients who underwent lumbar spine surgery at the Cleveland Clinic from 2013-2015 and completed an HCAHPS survey. The primary outcome of interest was a patient's Pain Management score, which is one of nine components of patient experience on the HCAHPS survey. A multi-variable regression model was developed to investigate the association between patient's Pain Management scores and the dose and duration of their post-operative pain medication regimen as well as several other co-variates.

Results: When all co-variates in the regression model are held constant, an increase of 30 morphine equivalents of pain medication per day is associated with a $38.13 \%$ (95\% CI:16.38-64.54\%) increase in the mean number of Pain Management points lost. Similarly, an increase in 1 week duration of pain medication is associated with $20.51 \%$ (95\% CI:6.88-36.00\%) increase in the mean number of Pain Management points lost. Figure 1 provides a graphical demonstration of example predictions from the final regression model.

Conclusion: The findings of this analysis suggest that postoperative opioid duration and dosage are negatively correlated with pain management scores on the HCAHPS survey in lumbar 
spine surgery patients. THis analysis suggests that increasing post-operative opioid prescriptions may not necessarily lead to improved pain management scores.

337 Elective Lumbar Spinal Fusion in Controlled HIV Patients Have Better Outcomes?

Karthik Madhavan, MD; chester John donally III, MD; Piyush Kalakoti, MD; Andrew Noel Livingstone, MS; Lee Onn Chieng, MD; Michael Y. Wang, MD, FACS; Steven Vanni, DO, DC

Introduction: Advancements in human immunodeficiency virus (HIV) detection and treatment have prolonged the life expectancy of those infected. No prior study has evaluated elective lumbar spinal surgery in HIV patients or patients who have not developed AIDS.

Methods: A retrospective analysis of the Nationwide Inpatient Sample (NIS; 2002-2011) was conducted to determine if differences exist between individuals with $(n=400)$ and without $(n=611,602)$ a diagnosis of asymptomatic HIV (no diagnosis of AIDS) with respect to perioperative complications surrounding elective lumbar spinal fusion surgery.

Results: The HIV-positive group had more males $(61.2 \%$ vs. $42.0 \%, \mathrm{p}<.001)$, younger $(47.01+9.13$ years vs. $54.91+$ 13.98years, $\mathrm{p}<.001)$, had higher rates of pre-existing COPD $(23.7 \%$ vs. $14.6 \%, \mathrm{p}<.001)$, but lower rates of obesity, hypertension, and diabetes $(\mathrm{p}<.001)$. While the asymptomatic HIV group had higher rates of an adverse post-operative event $(14.2 \%(\mathrm{~N}=57)$ vs. $10.4 \%(\mathrm{~N}=63,646) ; \mathrm{p}=0.015)$, only odds of respiratory complications (OR: 5.457) and death (OR: 40.267) attained sufficient statistical power $(>.80)$ to reliably interpret.It is unclear as to the reason for this high latter number due to the nature of database.

Conclusion: On the contrary to the previously published manuscripts in joint replacement and spine, there were considerably less complications noted with the HIV patients in the current study. Our study was intentionally limited to elective lumbar spine surgery in patients with "controlled HIV" unlike the previous publications. We believe our data sheds new light on elective spine surgery in HIV patients and might change the outlook of surgeons who are familiar with the older manuscripts citing grim outcomes.

338 Circumferential Correction of Thoracolumbar Deformity Secondary to Bacterial Spondylodiscitis

Mena G Kerolus, MD; Mazda K Turel, MD; Ricardo B.V. Fontes, MD, PhD

Introduction: Post-infectious deformity is a rare complication of osteomyelitis ofthe spine.Non-operative or limited operative treatment with debridement is usually futile due to destruction of the anterior column. Major reconstructive surgery may be a large undertaking with high morbidity.We describe our experience and outcomes with circumferential correction and fusion for patients with postinfectious deformity.

Methods: A retrospective review of 12 consecutive patients who underwent a three-column osteotomy for thoracolumbar deformity due to bacterial osteomyelitis during an 24-month period is reported. Preoperative data included ambulatory status,CCI,clinical and infectious data,and VAS scores. Complications are reported.The severity of their immediate postoperative condition is reflected by the SAPS II score. Outcome variables included VAS scores, ambulatory status, ability to care for self,return to work status and imaging.

Results: The mean age was $60.7 \pm 6.5$ years. All patients had refractory back pain,unable to ambulate and confirmed diagnosis of a spinal infection.The median CCI was 6(range, 1-8)and mean
VAS was $8.7 \pm$ 1.8.All patients underwent a 1 or 2 level 3-column osteotomy with an average of 9 fused levels.The mean EBL was $3300 \mathrm{ml}$.The median SAPS II score was 32(range,15-54).The median ICU and total hospital stay was 6 and 15 days respectively. The most common complication was intraoperative hypotension requiring vasopressor support in 6 patients.One patient died on POD7 from a pulmonary embolism.Postoperative VAS was reduced to $1.5 \pm 1.06$. All patients except two(preoperative paraplegia)regained ambulatory status at a mean follow-up of 6 months. Mean preoperative segmental kyphosis of $30 \pm 11$ degrees was corrected to $10 \pm 7$ degrees.

Conclusion: This study suggests that extensive circumferential reconstruction for deformity originating from bacterial discitis is effective in restoring these very sick patients to self-care and ambulatory status,with mortality and morbidity rates as expected from their physiological condition and the natural history of long fusions in debilitated older individuals.Extensive reconstruction is feasible and should be considered even during the subacute phase of these complex infections.

339 Preoperative Radiographic Predictors of Segmental Lordosis Correction After TLIF

Alexander Dru, MD; Ken Michael Porche, MD; Daniel J. Hoh, MD

Introduction: There is increased awareness of the importance of optimizing lordosis after lumbar fusion. Various interbody techniques may enhance postoperative lordosis by increasing anterior disc height. Transforaminal lumbar interbody fusion (TLIF) is a common approach, and results in varying degrees of lordosis correction. The purpose of this study is to determine preoperative radiographic factors that predict change in postoperative segmental lordosis after TLIF.

Methods: This study is a single surgeon retrospective review of 1-2 level TLIF. All patients underwent a bilateral facetectomy, $10 \mathrm{~mm}$ TLIF cage, and bilateral pedicle screw-rod construct. Preoperative $\mathrm{x}$-ray was assessed for preop segmental lordosis (Cobb angle); CT/MRI were used for anterior disc height, annular tear, Schmorl's node, Modic changes, bridging osteophytes, and vacuum disc. Postop segmental lordosis was measured on immediate postop x-rays. Change in lordosis was calculated by the difference in postop - preop Cobb angles at the TLIF level. Univariate/ multivariate regression analysis was performed to determine the relationship between preoperative radiographic factors and change in segmental lordosis.

Results: 137 disc levels in 103 patients were examined. Mean age was 62.3 years; $54 \%$ were women. All TLIF levels demonstrated improved change in segmental lordosis; mean change $=7.59$ degrees (range: 0.8-28). Preop annular tear was significantly associated with less change in lordosis $(\mathrm{p}=0.02)$. Greater preop lordosis $(\mathrm{p}=0.0001)$ and anterior disc height $(\mathrm{p}=0.01)$ also predicted less postop correction, which may represent a "ceiling effect." Vacuum disc on preop MRI trended towards better postop lordosis correction $(\mathrm{p}=0.13)$, but was not statistically significant.

Conclusion: TLIF is an effective approach for enhancing correction of segmental lordosis. Annular tear on preoperative imaging predicts less postoperative correction. Patients with already significant segmental lordosis (e.g. increased anterior disc height) on preop imaging also have less postoperative change. Vacuum disc phenomenon may prove to correlate with better postop lordosis. 
340 Fusion for High-grade Pediatric Lumbar Spondylolisthesis: A Short Case Series with Focus on the Importance of S2 Alar-iliac Screws for Pelvic Fixation as a Salvage Strategy in Revision Spondylolisthesis Cases.

Amey R Savardekar, MCh; Richard P Menger, MD, MPA; Devi Prasad Patra, MD, M.CH, MRCS; Anil Nanda, MD, FACS; Anthony H. Sin, MD

Introduction: Literature pertaining to failed fusions and revision pediatric spondylolisthesis surgery is scarce. Salvage strategies in the form of iliac screws for the commonly occurring S1 screw failures have been described; however, limitations exist. We illustrate the utilization of S2AI screw as a salvage strategy in revision pediatric spondylolisthesis surgery.

Methods: Three consecutive surgically managed pediatric high-grade spondylolisthesis patients were retrospectively analyzed. Pre-operative, post-operative and follow-up clinical and radiological data pertaining to the cases was retrieved from the hospital electronic database.

Results: All three patients were petite, pre-pubertal females (Risser grade I) presenting with symptomatic Meyerding grade IV spondylolisthesis at the L5-S1 level. As per the SDSG classification system, case 1 was type 6 ; while, cases 2 and 3 were type 5. Patients underwent successful operative management with wide decompression of neural structures, reduction of spondylolisthesis (to maximum possible extent) and posterior fusion. Case 1 had failed fusion at 12 months follow-up due to bilateral S1 screw breakage. The distal fixation at revision was achieved by utilizing $\mathrm{S} 2$-alar iliac screws as salvage strategy in order to get pelvic fixation into the construct.

Conclusion: Certain issues like incorporation of pelvic support in primary surgery remain contentious due to the thin built and prominent pelvic bones encountered in children. The S2 alar-iliac screw may be safely considered as a salvage procedure in cases of failed fusion or may even be incorporated to enhance the strength of the construct (by including pelvic support) in high grade pediatric spondylolisthesis in unbalanced spines.

341 Women Fare Best Following Surgery for Degenerative Lumbar Spondylolisthesis a Comparison of the Most and Least Satisfied Patients Utilizing Data from the Quality Outcomes Database

Andrew Kai-Hong Chan, MD; Erica Fay Bisson, MD, MPH, FAANS; Mohamad Bydon, MD; Steven D. Glassman; Kevin T. Foley, MD, FACS, FAANS; Eric A. Potts, MD; Christopher I. Shaffrey, MD, FACS; Mark Edwin Shaffrey, MD, FAANS, FACS; Domagoj Coric, MD; John J. Knightly, MD;

Introduction: Differing results from recent randomized control trials have established a need for clarification of groups that may benefit most from surgery for degenerative lumbar spondylolisthesis. This study compares the most and least satisfied patients following surgery for degenerative lumbar spondylolisthesis.

Methods: This was a retrospective analysis of a prospective, national longitudinal registry. 477 patients underwent surgery for grade 1 degenerative lumbar spondylolisthesis. The most satisfied and least satisfied patients were identified based on an answer of "1" and "4", respectively, on the North American Spine Society (NASS) Satisfaction Questionnaire 12-months post-operatively. Baseline demographics, clinical variables, surgical parameters, and follow-up outcomes were collected. PROs including numeric rating scale (NRS) back pain, NRS leg pain, Oswestry Disability Index (ODI), and EuroQoL-5D (EQ-5D) were collected at baseline, 3 months, and 12 months.
Results: $255(53.5 \%)$ patients were most satisfied and 26 $(5.5 \%)$ were least satisfied. As compared to the most satisfied patients, the least satisfied patients more often had coronary artery disease (CAD) (26.9\% vs. $12.2 \%, \mathrm{p}=0.04)$ and had higher body mass indices $(32.9 \pm 6.5$ vs. $30.0 \pm 6.0, p=0.02)$. In the multivariate analysis, female gender (OR 2.9, $\mathrm{p}=0.02$ ) was associated with the most satisfaction. Notably, ASA grade, smoking, psychiatric comorbidity, and employment status were not significantly associated with satisfaction. Despite having no significant differences at baseline, the most satisfied patients had significantly less NRS back- and leg pain scores, ODI, and a greater EQ-5D at 3- and 12 -months post-operatively ( $\mathrm{p}<0.001$ for all).

Conclusion: This study reveals that patient factors differ between patients that report the most and least satisfaction following surgery for degenerative lumbar spondylolisthesis. Patients reporting the least satisfaction tended to have CAD or were obese. Female gender was associated with the most satisfaction when adjusting for potential covariates. These findings highlight several key factors that may aid in expectation setting following surgery for degenerative lumbar spondylolisthesis.

342 Defining Usage and Clinical Outcomes Following Perioperative Fresh Frozen Plasma (FFP) Administration in the Spine Surgery Population

Timothy Y. Wang, MD; Taylor Elise Purvis, BA; Eric W. Sankey, MD; Camilo A. Molina, MD; Aladine A. Elsamadicy, BE; Steven M. Frank, MD; Daniel M. Sciubba, MD; C. Rory Goodwin, $\mathrm{MD}, \mathrm{PhD}$

Introduction: In the spine surgery population, utilization of fresh frozen plasma (FFP) is not well characterized. Additionally, risk factors and clinical outcomes following its administration are relatively unknown.

Methods: At a single large tertiary medical center, the surgical billing database was retrospectively queried for patients undergoing spinal surgery from a period of 2008 to 2015. A univariate analysis compared demographic data, comorbidities, surgical approach, preoperative laboratory values, estimated blood loss, blood transfusion, and postoperative complications between patients who received FFP intraoperatively and those who did not. To determine independent predictors of perioperative FFP administration and independent predictors of postoperative complications, a multivariate analysis was run on univariate variables that reached statistical significance $(\mathrm{p}<0.05)$.

Results: In total, 6,931 patients met inclusion criteria. One thousand seven $(14.5 \%)$ patients received perioperative FFP transfusion. In univariate analysis, older age, higher American Society of Anesthesiologists (ASA) grade, higher Charlson Comorbidity Index $(\mathrm{CCI})$, preoperative hemoglobin $<12$, preoperative international normalized ratio (INR) $=1.7$, higher estimated blood loss (EBL), and intraoperative crystalloid administration were associated with perioperative FFP administration. Those who received intraoperative FFP were more likely to experience infection, longer length of stay, and ischemic, respiratory, and renal complications. Following multivariate analysis, it was determined that $\mathrm{CCI}=4$, hemoglobin $<12, \mathrm{INR}=1.7$, higher $\mathrm{EBL}$, and blood and platelet transfusion were independent predictors of perioperative FFP administration. Fresh frozen plasma, ASA grade 3 or 4 , $\mathrm{CCI}=4$, and blood product transfusion were found to be an independent predictor of experiencing any complication.

Conclusion: Patients who have significant baseline medical comorbidities, anemia, and coagulopathy are at higher risk for receiving perioperative FFP. Perioperative FFP administration is an independent predictor of postoperative complications following spine surgery. 
343 Operating Microscope Utilization and Outcomes: A Comparison Between Specialties

Allen L Ho, MD; Arjun Vivek Pendharkar, MD; Paymon Rezaii; Eric S Sussman, MD; Anand Veeravagu, MD; John K. Ratliff, MD, FACS; Atman Desai, MD, MA

Introduction: There has been considerable debate about the utility of the operating microscope in common spine procedures and differences in utilization between neurosurgeons and orthopedic surgeons. We report on operating microscope utilization and outcomes between neurosurgeons and orthopedic surgeons for the two most common spine procedures: ACDF and lumbar discectomy.

Methods: A commercially available longitudinal database was used to identify patients undergoing ACDF or lumbar discectomy with or without microscope between 2007-2015. Propensity matching was performed to normalize differences between demographics and comorbidities in the two cohorts. Outcomes, complications and cost were subsequently analyzed with multivariate logistic regression.

Results: For ACDF a total of 91605 patients were identified for the 'macroscopic' group, while 9669 patients were identified for the microscope group. For lumbar discectomy a total of 42045 patients were macroscopic and 11172 were microscope. For macroscopic lumbar discectomy, length of stay was significantly increased amongst orthopedic surgeons (2.18 days versus 2.07 days, $\mathrm{P}<0.0003$ ), as was rate of revision surgery (OR 1.16 (1.04$1.28), \mathrm{P}<0.0067)$. Patients of orthopedic surgeons were also less likely to be discharged home (macroscopic OR 0.79 (0.72-0.87), $\mathrm{P}<0.0001$; microscopic OR 0.83 (0.70-0.99), $\mathrm{P}<0.0335)$. For $\mathrm{ACDF}$, there was no significant difference in outcomes between neurosurgeons and orthopedic surgeons. In terms of charges, orthopedic surgeons have significant increases total charges utilizing both macroscopic and microscopic techniques for lumbar discectomies $(\mathrm{P}<0.0001)$ and ACDF $(\mathrm{P}<0.0001)$.

Conclusion: The present study suggests that for lumbar discectomy, orthopedic surgeons that do not utilize the operating microscope have longer lengths of stay and increased rates of revision surgery. There was no difference in outcome measures studied between specialties with or without microscope utilization. However, orthopedic surgeons have higher total charges across the board for both these procedures.

344 Limited Post-Operative Dexamethasone Use Does Not Affect Lumbar Fusion: A Single Institutional Experience

Owoicho Adogwa, M.D., M.P.H; Syed Ibad Khalid; Victoria D Vuong; Daniel Lilly, B.S.; Shyam Desai; Ryan Khanna, MD; Shahjehan Ahmad; Josha Woodward, BS; Joseph S. Cheng, MD, MS

Introduction: To investigate the effect of limited exogenous dexamethasone use on bone fusion after instrumented lumbar arthrodesis. The use of exogenous dexamethasone during and after lumbar spine surgery remains controversial. The preponderance studies on this topic are primarily from animal models and little is known about the effects of exogenous dexamethasone use on fusion outcomes in human subjects undergoing lumbar arthrodesis.

Methods: Consecutive adult patients (18 years and older) undergoing one and two level lumbar decompression and fusion between January 2013 and December 2014 were reviewed for this study. Patients were dichotomized into one of two groups (A \& B) based on whether they received dexamethasone Group A: received dexamethasone; and Group B) no dexamethasone. Baseline char- acteristics, operative details, length of hospital stay, rates of wound infection as well as fusion rates at 1 year were gathered by direct medical record review. All patients enrolled in this study were followed for a minimum of 12 months after surgery.

Results: 165 consecutive patients undergoing 1- and 2-level fusions were included in the study. 58 patients received dexamethasone and 107 patients did not. The mean+/- SD age was similar between both cohorts( "Dexamethasone": 58.12+/-16.25 years, "no Dexamethasone": 61.00+/- 12.95, p=0.24). There was no difference in BMI between both cohorts("Dexamethasone": $27.67+/-5.28 \mathrm{~kg} / \mathrm{m} 2$, "no Dexamethasone": $29.40+/-7.31 \mathrm{~kg} / \mathrm{m} 2$, $\mathrm{p}=0.24)$. There was no significant difference in the prevalence of smoking between both patient cohorts $(\mathrm{p}=0.72)$. The length of inhospital stay was similar between both cohorts(Dexamethasone": 4.08+/- 3.44 days vs. "no Dexamethasone": 4.50 +/- 2.85 days, $\mathrm{p}=0.43$ ). The incidence of post-operative infections was similar between both cohorts. At 12 months after surgery, $70 \%$ of patients in the dexamethasone cohort had radiographic evidence of bony fusion compared to $73 \%$ of patients in the no-dexamethasone $\operatorname{cohort}(\mathrm{p}=0.68)$.

Conclusion: Our study suggests that a limited exposure to exogenous dexamethasone after lumbar spine fusion may not be associated with a lower fusion rate. Prospective randomized control trials are needed to corroborate our findings.

345 Hemoglobin A1c as a Predictor of Surgical Site Infection Following Single Level Lumbar/Lumbosacral Posterior Fusion in Patients with Diabetes

Jun Seok Lee, M.D.; Dong Wuk Son; Sang Weon Lee; Geun Sung Song

Introduction: Diabetes mellitus (DM) is a prevalent disease of glucose dysregulation that has been associated with an increased risk for postoperative infection following spine surgery. The status of preoperative blood glucose management may affect the risk of surgical site infection (SSI). In the present study, we evaluated the association between preoperative glycemic control as indicated by hemoglobin $\mathrm{A} 1 \mathrm{c}(\mathrm{HbA} 1 \mathrm{c})$ in patients with diabetes and incidence of postoperative SSI following single level lumbar/ lumbosacral posterior fusion.

Methods: From January 2009 to December 2015, total 92 patients who underwent single level lumbar/lumbosacral posterior fusion with diabetes and had preoperative $\mathrm{HbA} 1 \mathrm{c}$ recorded within 4 weeks of surgery were included in the study. Patients were divided into two groups according to whether they had SSI, and then demographic/clinical data were compared. A receiver operating characteristic (ROC) and area under the curve (AUC) analysis was conducted to define the cut-off value of HbA1c above which the risk of SSI was significantly increased. Including this value, potential variables were verified by multiple logistic regression analysis.

Results: Twenty four patients were treated for SSI and 68 patients maintained noninfectious condition within 1 year. Three of the $24(12.5 \%)$ patients developed SSI in the deep layer requiring operative irrigation and debridement. The preoperative HbA1c value was significantly higher in patients with SSI $(6.8 \%)$ than in those without SSI $(6.0 \%$; $=0.008)$. The results of ROC analysis determined that $\mathrm{HbA} 1 \mathrm{c}=6.9 \%$ could serve as a threshold for significantly increased risk of SSI ( $\mathrm{p}=0.003, \mathrm{AUC}=0.708$, sensitivity $=62.5 \%$, specificity $=70.6 \%$ ). After adjusting for confounding factors, there was a significant association between preoperative HbA1c and occurrence of SSI $(\mathrm{p}=0.008, \mathrm{OR}=4.500)$.

Conclusion: In patients with diabetes, the preoperative glycemic control as indicated by $\mathrm{HbA} 1 \mathrm{c}$ is an independent risk factor for SSI following single level lumbar/lumbosacral posterior fusion. 
Particularly when preoperative HbA1c exceeded $6.9 \%$, the risk of SSI significantly increased.

346 Neurological Outcomes in Spontaneous Spinal Epidural Abscesses: A Systematic Review and Meta-analysis of Data from 1980 through 2017

Timothy Y. Wang, MD; Stephen C Harward, MD; Vadim Tsvankin, MD; Hannah Bell, BS; Lefko Charalambous, BS; Syed Adil, BS; Dario J. Englot, MD, PhD; C. Rory Goodwin, MD, PhD; Isaac O. Karikari, MD; Robert E. Isaacs, MD; Max Ostrinsky Krucoff, MD

Introduction: Current literature on spinal epidural abscesses (SEA) lacks large-scale data characterizing prognostic factors and surgical indications. Therefore, we performed a systematic review and the first quantitative meta-analysis of all available SEA literature published between 1980 and 2017.

Methods: PubMed was queried for studies reporting neurological outcomes for patients undergoing conservative or surgical management for spontaneous SEA. Inclusion criteria included outcomes data measured $>6$ months after presentation, $>10$ human subjects, and diagnosis by MRI or CT-myelogram. Where available, demographic data, abscess location, comorbidities, pretreatment neurological deficits, treatment methods, bacterial speciation, neurological outcomes, and complications were extracted from each study. Potential outcome predictors represented by continuous variables were compared using Student's t-test and categorical variables were compared using Pearson's chi-squared. Variables potentially associated with outcome $(\mathrm{p}=0.05)$ were subjected to meta-analysis using Cochran Mantel Haenszel testing to calculate odds ratios (ORs) and $95 \%$ confidence intervals (CIs).

Results: 20 studies totaling 808 patients met inclusion criteria. $456(56.3 \%)$ were treated with surgery and antibiotics and 353 (43.7\%) patients were managed with antibiotics alone. Neither surgical intervention (OR 1.01,95\% CI 0.40-2.59), lumbosacral location (OR 1.51, 95\% CI 0.23-9.79), nor neurological deficit on presentation (OR $0.88,95 \%$ CI $0.40-1.92)$ were significantly associated with good (stable or improved) or bad (worsened) neurological outcome, whereas delayed surgery was significantly associated with bad outcome (OR 0.01, 95\% CI 0.02-0.62). Cervicothoracic location approached significance for predicting bad outcome (OR 0.41, 95\% CI 0.15-1.09).

Conclusion: Contrary to most common practice, current literature does not definitively support or oppose surgical intervention in all SEA cases. Therefore, the decision to operate must be made on an individual case-by-case basis with the goals of preventing neurological decline, obtaining source control after failed conservative treatment, or restoring spinal stability. SEA literature would benefit from prospectively collected data to assess for factors that might affect surgical decision making and/or outcomes.

347 A Retrospective Study of Operative Outcomes for Patients with Spinal Epidural Abscess

Leonard Keller, BS; Joseph E Tanenbaum, BA; Vincent J Alentado, MD; Bryan S Lee, MD; Amy S. Nowacki, PhD; Thomas E. Mroz, MD; Edward C. Benzel, MD; Michael P. Steinmetz, MD

Introduction: A spinal epidural abscess (SEA) is a serious condition that can be treated with antibiotics alone or with decompression surgery combined with antibiotics. The objectives of this study were to assess the clinical outcomes of SEA after surgical management and to identify patient-level factors that are associated with outcomes following surgical decompression of SEA.

Methods: A retrospective analysis of 152 consecutive patients that presented to the Cleveland Clinic with SEA and that were treated with surgery between 2010 and 2015 was performed. Post-operative pre-discharge ASIA scores, 6-month follow-up encounter ASIA scores, more than one SEA surgery, and death during SEA surgery were the primary outcomes. Fisher's exact and Wilcoxon rank-sum tests were used to determine the association between patient-level factors and outcomes.

Results: 152 patients were treated using surgical decompression and antibiotics during the study period. None of the patients died during surgery. A second SEA surgery was required in $8 \%$ of patients. A comparison of the pre-operative ASIA and postoperative pre-discharge ASIA scores showed that $49 \%$ of patients maintained an ASIA score of E or improved, $45 \%$ remained at their pre-operative status, and $6 \%$ worsened. Among a subset of patients $(n=36)$ for whom a $6 \pm 2$ month follow-up encounter occurred, $75 \%$ maintained an ASIA score of $\mathrm{E}$ or improved, $19 \%$ remained at their pre-operative status, and $6 \%$ worsened. Both the presence and longer duration of pre-operative paresis was associated with an increased risk of remaining at the same ASIA score or worsening at the post-operative pre-discharge encounter (both $\mathrm{p}<0.001)$.

Conclusion: Surgical decompression as part of the management of SEA can contribute to improving or maintaining ASIA scores in most patients. The presence and duration of pre-operative paresis are prognostic for poorer outcomes, suggesting that rapid surgical intervention before it develops may lead to improved outcomes.

348 Post-operative Drain Use in Patients Undergoing Decompression and Fusion: Incidence of Complications and Symptomatic Hematoma

Owoicho Adogwa, M.D., M.P.H; Aladine A. Elsamadicy, BE; Amanda Sergesketter; Victoria D Vuong; Syed Ibad Khalid; Joseph S. Cheng, MD, MS; Carlos Antonio Bagley, MD; Isaac O. Karikari, MD

Introduction: Surgical drains are commonly used after spine surgery to minimize the risk of infection and hematoma formation. The aim of this study is to assess the rate of post-operative surgical site infection and hematoma formation in adult deformity patients undergoing spinal decompression and fusion with and without the use of a sub fascial drain.

Methods: The medical records of 369 adult spinal deformity patients $(=18$ years old) undergoing elective spinal decompression and fusion at a major academic institution from 2005 to 2015 were reviewed. We identified $332(90 \%)$ who had a post-operative drain and $37(10 \%)$ who did not have a postoperative drain (No-Drain: $\mathrm{n}=37$; Drain-Use: $\mathrm{n}=332$ ). Patient demographics, comorbidities, intra- and post-operative complication rates were collected for each patient. The primary outcome investigated in this study was the rate of post-operative complications, specifically surgical site infections and hematomas.

Results: Patient demographics and comorbidities were similar between both cohorts, with the body mass index (BMI) increased in the Drain-use cohort (No-Drain: $27.2 \mathrm{~kg} / \mathrm{m} 2$ vs. Drain-Use: 29.4 $\mathrm{kg} / \mathrm{m} 2, \mathrm{p}=0.04)$. The median number of levels fused, estimated blood loss, and need for allogenic blood transfusions were significantly higher in the Drain-Use cohort compared to the No-Drain cohort. Length of hospital stay (LOS) was significantly higher for the Drain-use cohort compared to the No-Drain cohort (6.3 days vs 3.5 days, $\mathrm{p}<0.0001)$. The incidence of SSI's and hematomas was similar between both cohorts, $(\mathrm{p}=0.45$ and $\mathrm{p}=0.41$, respectively). The 30-day readmission rate was similar between both cohorts (No-Drain: $13.5 \%$ vs. Drain-Use: $13.3 \%$, $\mathrm{p}=0.96$ ). There were no significant differences in the prevalence of 30-day wound 
dehiscence $(\mathrm{p}=0.37)$, draining wound $(\mathrm{p}=0.64)$, and need for reoperation $(\mathrm{p}=0.32)$.

Conclusion: Our study suggests that the use of a postoperative drain in patients undergoing spinal decompression and fusion may not reduce surgical site infection and hematoma rates.

349 Complications Profile in Patients with Chronic Kidney Disease Undergoing Lumbar Spine Decompression and Fusion

Owoicho Adogwa, M.D., M.P.H; Aladine A. Elsamadicy, BE; Amanda Sergesketter; Deborah Oyeyemi, B.A.; Diego Galan, B.S.; Victoria D Vuong; Syed Ibad Khalid; Daniel Lilly, B.S.; Shyam Desai; Joseph S. Cheng, MD, MS; Carlos Antonio Bagley, MD; Isaac O. Karikari, MD

Introduction: Chronic Kidney Disease (CKD) has been shown to have significant implications on surgical outcomes. However, little is known about the impact that CKD has on patients undergoing spine surgery. The aim of this study is to investigate the postoperative complications profile in patients with CKD undergoing lumbar decompression and fusion for deformity correction.

Methods: The medical records of 293 adult spine deformity patients $(=18$ years old) undergoing elective lumbar spine decompression and fusion at a major academic institution from 2006 to 2015 were reviewed. We identified $18(6.1 \%)$ who had a clinical diagnosis of CKD (CKD: $n=18$; No-CKD: $n=275$ ). Patient demographics, comorbidities, intra- and post-operative complication rates were collected for each patient. The primary outcome investigated in this study was the rate of post-operative complications.

Results: Patient demographics and comorbidities were mostly similar between both cohorts, including age, gender, and BMI, Table 1 . The CKD cohort had a significantly higher prevalence of hypertension, hyperlipidemia, and anemia compared to the No-CKD cohort, Table 1 . The median number of fusion levels, length of surgery and estimated blood loss were similar between both cohorts, Table 2 . The postoperative complication profile was significantly different between both cohorts, with the CKD cohort having significantly higher proportion of patients transferred to the ICU $(52.9 \%$ vs. $29.3 \%, \mathrm{p}<0.001)$, experiencing episodes of delirium ( $27.8 \%$ vs. $8.4 \%, \mathrm{p}=0.007)$, UTI $(27.8 \%$ vs. $6.9 \%$, $\mathrm{p}=0.0002)$, and DVT ( $5.6 \%$ vs. $0.4 \%, \mathrm{p}=0.01)$, Table 3 . Although not significant, the CKD cohort had a 2-fold higher rate of 30-day readmissions compared to the non-CKD cohort (CKD: $27.8 \%$ vs. No-CKD: $12.7 \%, \mathrm{p}=0.07)$, Table 3 .

Conclusion: Our study suggests that patients undergoing spinal decompression and fusion with CKD may experience higher complication rates than patients without CKD.

350 Defining the Minimum Clinically Important Difference for Grade I Degenerative Lumbar Spondylolisthesis: Insights from the Quality Outcomes Database

Anthony L. Asher MD, FACS; Panagiotis Kerezoudis; Andrew Kai-Hong Chan MD; Praveen V. Mummaneni MD; Erica Fay Bisson MD, MPH, FAANS; Steven D. Glassman; Kevin T. Foley MD, FACS, FAANS; Jonathan Slotkin MD; Eric A. Potts MD; Mark Edwin Shaffrey MD, FAANS,

Introduction: The concept of minimum clinically important difference (MCID) is considered the new standard for determining the effectiveness of a given treatment and describing patient satisfaction in response to that treatment. Herein, we sought to determine the MCID associated with surgical treatment for degenerative lumbar spondylolisthesis.

Methods: We queried the Quality Outcomes Database registry from July 2014 through December 2015 for patients undergoing posterior lumbar surgery for grade I degenerative spondylolisthesis. Recorded patient reported outcomes included Oswestry Disability Index (ODI), EQ-5D, numeric rating scale (NRS)-leg pain and -back pain. Anchor-based (using the NASS satisfaction scale) and distribution-based (half a standard deviation, small Cohen's effect size, standard error of measurement and minimum detectable change (MDC)) methods were used to calculate the MCID for each PRO.

Results: A total of 441 patients (80 laminectomies alone, 361 fusions) from 11 participating sites were included in the analysis. Change in functional outcomes scores between baseline and 1-year were $23.5 \pm 17.4$ points for ODI, $0.24 \pm 0.23$ for EQ-5D, $4.2 \pm$ 3.5 for NRS-LP, and $3.7 \pm 3.2$ for NRS-BP. The different calculation methods generated a range of MCID values for each PRO: 3.3 to 26.5 points for ODI, 0.04 to 0.3 points for EQ-5D, 0.6 to 45.5 points for NRS-leg pain and 0.5 to 4.12 points for NRS-back pain. The MDC approach appeared to be the most appropriate for calculating MCID because it provided a threshold greater than the measurement error and was closest to the average change difference between the satisfied and not satisfied patients. On subgroup analysis, the MCID thresholds for laminectomy alone patients were comparable to those undergoing arthrodesis as well as the entire cohort.

Conclusion: The MCID for PROs was highly variable depending on calculation technique. The MDC seems to be a statistically and clinically sound method for defining the appropriate MCID value for patients with grade I degenerative lumbar spondylolisthesis. Based on this method, the MCID values are 14.3 points for ODI, 0.2 points for EQ-5D, 1.7 points for leg pain, and 1.6 points for back pain.

351 Surgical Factors Which Affect Patient Reported Outcomes (PRO) Completion 1 Year After Spine Surgery: Analysis from the Michigan Spine Surgery Improvement Collaborative (MSSIC)

Hesham Mostafa Zakaria, MD; Michael Bazydlo, MS; Shujie Xiao, MS; Lonni Schultz, PhD; David Nerenz, PhD; Muwaffak Abdulhak, MD, FRCS; Jason M. Schwalb, MD, FAANS, FACS; Victor W. Chang, MD; For the MSSIC Investigators

Introduction: The Michigan Spine Surgery Improvement Collaborative (MSSIC) is a statewide multicenter quality improvement registry. 1 Since missing data can affect registry results,2, 3 we used MSSIC to find surgical characteristics that affect the completion of patient-reported outcome surveys (PROs) at 90 days and 1 year.

Methods: 24404 patients from 26 MSSIC hospitals who had lumbar (17813 patients) or cervical (6591 patients) surgery were included. Multivariate logistic regression models of patient pathology were constructed to identify risk factors for failure to complete scheduled PRO surveys.

Results: For cervical spine patients, pain location (neck vs arm), myelopathy, multi-level surgery, any complication, anterior or posterior approach, outpatient or inpatient encounter, and length of stay did not affect PRO response at $90 \mathrm{~d}$ or $1 \mathrm{yr}$. Only discharge to rehab increased completion rates at $90 \mathrm{~d}(\mathrm{p}=0.002)$, but not $1 \mathrm{yr}(\mathrm{p}=0.547)$. For lumbar spine, patients with spondylolisthesis or stenosis (vs herniated disc) had a greater response rate at $90 \mathrm{~d}(\mathrm{p}<0.001, \mathrm{p}=0.043$, respectively) and $1 \mathrm{yr}(\mathrm{p}<0.001$, $\mathrm{p}=0.011$, respectively). Patients with leg (vs back) pain had a greater response only at $1 \mathrm{yr}(\mathrm{p}=0.017)$. Patients with multi-level (vs single-level) surgery had an increased response only at $1 \mathrm{yr}$ $(p=0.003)$. Patients who underwent fusion were more likely to respond at $90 \mathrm{~d}(\mathrm{p}<0.001)$ but not $1 \mathrm{yr}(\mathrm{p}=0.747)$. Discharge to rehab increased response at $90 \mathrm{~d}(\mathrm{p}<0.001)$ and $1 \mathrm{yr}(\mathrm{p}=0.038)$. 
Any complication, hospital stay, and claudication did not affect the PRO response rate.

Conclusion: A multivariate analysis from a multicenter prospective database identified surgical factors that affect PRO follow-up up to 1yr. This information can be helpful for imputing missing PRO data, and could potentially be used to strengthen data derived from large prospective databases. More studies are required to elucidate why these specific factors affect PRO; for example, patients with less complex pathology and simpler procedures may follow-up less due to the nature of their procedures.

352 Reduced In-patient Narcotic Consumption and Hospital Stay with ERAS ${ }^{\circ}$ Transforaminal Lumbar Interbody Fusion (TLIF): Comparison to Conventional Minimally-Invasive TLIF

John Paul George Kolcun, BS; Karthik Madhavan, MD; Hsuan-Kan Chang, MD; Timur Urakov, MD; Jay Grossman, MD; Michael Y. Wang, MD, FACS

Introduction: The concept of Enhanced Recovery After Surgery (ERAS $\AA$ ) is relatively new to spine surgery. Here, we describe the impact of an ERAS ${ }^{\circledR}$ protocol on in-hospital narcotic consumption following lumbar spinal fusion.

Methods: We conducted a retrospective review of patients with 1- or 2-level degenerative lumbar spine disease who underwent ERAS ${ }^{\circledR}$ awake endoscopic transforaminal lumbar interbody fusion (TLIF) with long-acting depoform bupivacaine, or conventional minimally-invasive (MIS) TLIF with general anesthesia, including medical records and radiographic images. Patients' general demographic data, in-hospital narcotic dosage (converted to morphine equivalent), and hospital length of stay were collected and compared between groups.

Results: The first 50 patients who underwent ERAS ${ }^{\circledR}$ TLIF (ERAS group) were compared to a series of 50 patients who underwent conventional MIS TLIF (MIS group). Demographics and preoperative functional status were similar between groups. The ERAS group had significantly reduced operative time $(106.8 \pm 36.5$ minutes vs. $162.1 \pm 44.4, \mathrm{p}<0.01)$ and blood loss $(32.4 \pm 34.1 \mathrm{~mL}$ vs. $124.0 \pm 90.9, \mathrm{p}<0.01)$. Postoperative hospital stay was significantly reduced in the ERAS group ( $1.0 \pm 0.9$ days vs. $3.5 \pm 1.4$ days, $\mathrm{p}<0.01)$. The average total narcotic dosage throughout the entire in-hospital period was significantly reduced in the ERAS group compared to the MIS group (64.1 \pm 77.9 MME vs. 194.6 \pm 153.4 MME, $\mathrm{p}<0.01$ ). Average daily narcotic consumption was also remarkably lower in the ERAS group $(\mathrm{p}<0.01)$. As the average length of hospital stay was only 23 hours in the ERAS group, meaningful comparison of daily narcotic consumption was only possible for postoperative days 0 and 1 .

Conclusion: This study demonstrates a significant decline in the consumption of narcotic medication and the hospital length of stay in patients undergoing ERAS ${ }^{\circledR}$ TLIF for 1- or 2-level degenerative lumbar spine disease.

353 Posterolateral Fusion (PLF) vs. Transforaminal Lumbar Interbody Fusion (TLIF) for Spondylolisthesis: A Systematic Review and Meta-Analysis

Jay M Levin, BA; Joseph E Tanenbaum, BA; Michael P. Steinmetz, MD; Thomas E. Mroz, MD; Samuel C Overley, MD

Introduction: Lumbar fusion is an effective treatment for lumbar spondylolisthesis, however the current literature provides insufficient evidence to recommend an optimal fusion strategy. We sought to compare the clinical outcomes, fusion rates, blood loss, and operative times between open posterolateral lumbar fusion (PLF) alone and open transforaminal lumbar interbody fusion + posterolateral fusion (TLIF) for spondylolisthesis.

Methods: A literature search of three electronic databases was performed to identify investigations comparing PLF alone versus PLF + TLIF for treatment of lumbar spondylolisthesis. The summary effect size was assessed from pooling observational studies for each of the outcome variables. Studies were weighted based on the inverse of the variance. Effect sizes from the meta-analysis were then compared to data from the RCTs to assess for congruency in outcomes.

Results: Initial literature search yielded 282 unique studies. Seven met our inclusion criteria and were included in our qualitative analysis. Five observational studies were included in our quantitative meta-analysis. The pooled fusion success rates were $84.7 \%(100 / 118)$ in the PLF group and $94.3 \%(116 / 123)$ in the TLIF group. Compared to TLIF, PLF patients had lower odds of achieving fusion (Odds Ratio [OR] 0.33, 95\% confidence interval [CI] 0.130 .82 ). For back pain, the point estimate for the effect size was -0.27 (95\% CI -0.43 to -0.10$)$, in favor of the TLIF group. For ODI, the pooled estimate for the effect size was -3.73 (95\% CI -7.09 to -0.38 ), in favor of the TLIF group. Operative times were significantly shorter in the PLF group. Our meta-analysis results were consistent with RCTs in favor of TLIF for achieving radiographic fusion and greater improvement in ODI and back pain.

Conclusion: Our results demonstrate that for patients undergoing fusion for spondylolisthesis, TLIF is superior to PLF with regards to achieving radiographic fusion and clinical improvement in disability and back pain.

354 Blood Storage Duration and Perioperative Outcomes in Spine Surgery

Taylor Elise Purvis, BA; C. Rory Goodwin, MD, PhD; Camilo A. Molina, MD; Steven M. Frank, MD; Daniel M. Sciubba, MD

Introduction: Despite retrospective studies that have shown that longer packed red blood cell (PRBC) storage duration worsens patient outcomes, randomized clinical trials have found no difference in outcomes. However, no studies have examined the impact of giving the oldest blood (28 days-old or more) on morbidity within spine surgery. Our objective in this study was to describe the association between storage duration of PRBCs and perioperative adverse events in patients undergoing spine surgery at a tertiary care center.

Methods: The surgical administrative database at our institution was queried for patients transfused with PRBCs who underwent spine surgery between December 4, 2008 and June 26, 2015. Patients undergoing spinal fusion, tumor-related surgeries, and other identified spine surgeries were included. Patients were divided into two groups based on storage duration of blood transfused: exclusively $<=28$ days' storage or exclusively $>28$ days' storage. The primary outcome was composite in-hospital morbidity, which included: (1) infection, (2) thrombotic event, (3) renal injury, (4) respiratory event, and/or (5) ischemic event.

Results: In total, 1,141 patients who received a transfusion were included for analysis in this retrospective study; 710 were transfused exclusively with PRBCs less than or equal to 28 days' storage and 431 exclusively with PRBCs $>28$ days' storage. Perioperative complications occurred in 119 patients $(10.4 \%)$. Patients who received blood stored for $>28$ days had higher odds of developing any one complication (odds ratio [OR] $=1.82 ; 95 \%$ confidence interval $[\mathrm{CI}], 1.20-2.74 ; \mathrm{P}=0.005)$ even after adjusting for competing perioperative risk factors.

Conclusion: Blood stored for $>28$ days is independently associated with higher odds of developing perioperative complications in patients transfused during spinal surgery. Our results suggest 
that blood storage duration may be an appropriate parameter to consider when developing institutional transfusion guidelines that seek to optimize patient outcomes.

355 Effect of Percent Change in Blood Hemoglobin Level on Morbidity in Spine Surgery Patients

Taylor Elise Purvis, BA; C. Rory Goodwin, MD, PhD; Camilo A. Molina, MD; Steven M. Frank, MD; Daniel M. Sciubba, MD

Introduction: Several studies in surgical fields have demonstrated the importance of considering delta hemoglobin (deltaHb) in the formula for appropriate perioperative blood transfusions, where deltaHb is the difference between a patient's preoperative $\mathrm{Hb}$ and nadir $\mathrm{Hb}$ concentration during a hospital stay. In this study, we sought to determine the perioperative clinical outcomes associated with percent deltaHb as an independent factor among spine surgery patients.

Methods: Patients who underwent spine surgery at our institution between December 4, 2008 and June 26, 2015 were eligible for this retrospective study. Patients undergoing the following procedures were included: atlantoaxial fusion, subaxial anterior cervical fusion, subaxial posterior cervical fusion, anterior lumbar fusion, posterior lumbar fusion, lateral lumbar fusion, excision of intervertebral disc, and excision of spinal cord lesion. Data on transfusion were obtained from a prospectively collected anesthesia data management system. Data on postoperative hospital transfusions were obtained through a Web-based intelligence portal. Percent deltaHb was defined as: ((first $\mathrm{Hb}$ - nadir $\mathrm{Hb}$ )/first $\mathrm{Hb}$ ) $\mathrm{x}$ 100. Clinical outcomes included in-hospital morbidity and length of stay associated with percent deltaHb.

Results: A total of 3,949 patients who underwent spine surgery were identified. A total of 1204 patients $(30.5 \%)$ received at least one unit of packed red blood cells (PRBC). The median nadir $\mathrm{Hb}$ level was $10.6 \mathrm{~g} / \mathrm{dL}$ (IQR, 8.7-12.4 g/dL), yielding a mean percent deltaHb of $23.6 \%(\mathrm{SD}=15.4 \%)$. Perioperative complications occurred in 234 patients $(5.9 \%)$ and were more common in patients with a larger percent deltaHb $(\mathrm{P}=0.017)$. Hospital-related infection, which occurred in 60 patients $(1.5 \%)$, was also more common in patients with a greater percent deltaHb $(\mathrm{P}=0.001)$.

Conclusion: Percent deltaHb is independently associated with a higher risk of developing any one perioperative complication and hospital-related infections. Our results suggest that percent deltaHb may be a useful measure to identify patients at risk for adverse perioperative events.

356 Quantification of Computational Geometric Congruence in Surface-based Registration for Spinal Intraoperative Three-dimensional Navigation

Daipayan Guha, MD; Raphael Jakubovic, MSc; Victor Xiao Dong Yang, MD, PhD, MSc, BSc

Introduction: Computer-assisted navigation (CAN) may guide spinal instrumentation, and requires alignment of patient anatomy to imaging. Iterative-Closest-Point algorithms register anatomical and imaging datasets, which may fail in the presence of significant geometric symmetry (congruence), leading to failed registration or inaccurate navigation. We computationally quantify geometric congruence in posterior spinal exposures, and identify predictors of potential navigation inaccuracy.

Methods: Midline posterior exposures were performed from C1-S1 in four human cadavers. An optically-based CAN generated surface maps of the posterior elements at each level. Maps were reconstructed to include bilateral hemilamina, or unilateral hemilamina with/without the base of the spinous process. Maps were fitted to symmetrical geometries (cylindrical/spherical/ planar) using computational modelling, and the degree of model fit quantified based on the ratio of model inliers to total points. Geometric congruence in a clinical setting was assessed similarly, in 11 patients undergoing midline exposures in the cervical/thoracic/lumbar spine for posterior instrumented fusion.

Results: In cadaveric testing, increased cylindrical/spherical/ planar symmetry was seen in the subaxial cervical spine relative to the high-cervical and thoracolumbar spine $(p<0.001)$. Inclusion of the base of the spinous process decreased symmetry independent of spinal level $(\mathrm{p}<0.001)$. Registration with bilateral vs. unilateral hemilamina did not significantly reduce geometric symmetry. In clinical testing, increased cylindrical/spherical/planar symmetry was again seen in the subaxial cervical spine relative to the thoracolumbar spine $(\mathrm{p}<0.001)$, and in the thoracic spine relative to the lumbar spine $(\mathrm{p}<0.001)$. Symmetry in all geometries was decreased by $20 \%$ with inclusion of the base of the spinous process vs. without.

Conclusion: Geometric congruence is most evident at $\mathrm{C} 1$ and the subaxial cervical spine, warranting greater vigilance in navigation accuracy verification. At all levels, inclusion of the base of the spinous process in unilateral registration decreases the likelihood of geometric symmetry and navigation error, important for minimally-invasive unilateral approaches.

357 Cost-effectiveness of an Adjunctive Bone-anchored Annular Closure Device Versus Conventional Lumbar Discectomy in Treating Lumbar Disc Herniations

Jared Ament, MD, MPH; Bart Thaci, MD; Edwin Kulubya, MD; Zhuo Yang, MS; Wellington Hsu, MD; Kee Kim, MD

Introduction: Lumbar herniation patients with large annular defects who undergo discectomy appear to be at high risk for reherniation, with concomitant poor outcomes, reoperations and incremental costs. The cost-effectiveness of a bone-anchored annular closure device (ACD) compared to conventional discectomy (Control) was therefore evaluated.

Methods: The analyzed population included both the Control and ACD-treated cohorts from a multicenter prospective RCT designed to assess safety and efficacy of the ACD. Patient follow-up visits were at 6 weeks and 3, 6, 12, 24, 36, 48, and 60 months postoperatively. Health states were created by projecting visual analogue scale (VAS) onto Oswestry Disability Index (ODI). Direct costs were calculated based on pertinent diagnosis delated groups (DRGs), post-operative medications, reoperations and surgery-related complications by applying inpatient 2017 Medicare rates to type/frequency of these measures. Quality of life was determined using SF-36 and SF-6D captured during the RCT. Final input parameters were used to inform a cohort Markov model based (Figure 1). The resulting incremental cost-effectiveness ratio (ICER) in units \$/QALY (quality adjusted life year) was then compared to accepted willingness-to-pay (WTP) thresholds.

Results: A strong correlation $(\mathrm{R} 2=0.826, \mathrm{P}<0.001)$ was found by projecting VAS onto ODI. From a healthcare perspective (direct costs only) ACD had an average of 3.50 QALYs gained after 60-months compared to 3.40 QALYs for the control group. ACD was associated with $\$ 1,824$ greater average cost. The ICER was therefore $\$ 18,544$ per QALY at five years. From a societal perspective (indirect costs included), ACD costs, on average, \$ 5,118 less than control, resulting in an ICER of negative $\$ 52,063$ per QALY at five years.

Conclusion: The 5 year ICER of ACD compared with the traditional lumbar discectomy is lower than commonly accepted WTP-thresholds. Accounting for the societal impact produces a negative ICER, suggesting that ACD could substantially improve 
the cost-effectiveness of lumbar discectomy, particularly high-risk patients.

358 Incidence of Preoperative Motor Weakness and Recovery after Spinal Surgery

Nathan John Lee, BS; Jeremy Steinberger, MD; Samuel K Cho, MD; John M. Caridi, MD

Introduction: The postoperative course of patients who present with preoperative neurological deficits is not well-documented. It is unclear if duration of preoperative deficit affects the likelihood and pace of neurologic recovery. It is also nebulous whether age, sex, diagnosis, type of surgery, severity of weakness, physical therapy, and ambulation play a role.

Methods: A retrospective evaluation of a cohort of 705 patients from March 2011 to July 2014 who underwent spine surgery by two spine surgeons. Data collected included age, sex, presence of neurological deficit preoperatively, severity of deficit, number of involved muscle groups, comorbidities, diagnosis, type of surgery, degree of recovery, time to recovery, follow up physical therapy, and days to ambulate. Neurologic examinations were recorded at every visit according to the Frankel grading system.

Results: 705 patients were studied from 3/2011-7/2014. Of these, 222 patients had a neurologic deficit prior to surgery. 16 patients were excluded from the study due to loss of follow up. Patients presenting with neurological deficit were 56 years old on average; $51.9 \%$ were male, and the average deficit motor grade was 3.3/5 on the Frankel motor score. The median time to recovery was 18 days. $92 \%$ of patients had some improvement from their preoperative Frankel motor score $80 \%$ a full recovery of function, $20 \%$ a partial improvement. The duration of deficit prior to surgery did not affect likelihood of postoperative recovery of function. Patients who went to acute rehabilitation were more likely to improve their neurologic function and walking sooner after surgery was associated with recovery of neurologic function.

Conclusion: Spine surgery has a high rate of improving preoperative neurologic deficits. Duration of deficit preoperatively does not affect recovery. The median time to recovery is 18 days.

359 Tailoring Selection of Transforaminal Interbody Spacer Based on Biomechanical Characteristics and Surgical Goals: Evaluation of an Expandable Spacer

Jakub Godzik, MD, MSc; Jennifer Lehrman, BSE, MS; Anna Newcomb; Alex Whiting, MD; Ramkumar Menon, MBBS, MS, DNB, MCh; Brian P. Kelly, PhD; Laura A. Snyder, MD

Introduction: Transforaminal lumbar interbody fusion (TLIF) is commonly used for lumbar fusion, with many indications, including foraminal decompression, stabilization, and improvement of segmental lordosis. While many options exist, surgical success is contingent on matching design strengths with surgical goals. The study objective was to investigate the effects of a novel expandable interbody spacer and two traditional spacer designs in regards to stability, compressive stiffness, foraminal height, and segmental lordosis.

Methods: Standard nondestructive flexibility tests $(7.5 \mathrm{Nm})$ were performed on 7 cadaveric lumbar specimens to assess intervertebral stability of 3 types of TLIF spacers with posterior screw rod fixation (PSR) bilaterally at L4/L5. Stability was determined as mean range of motion (ROM) in flexion/extension (F/E), lateral bending (LB), and axial rotation (AR). Axial compressive loading(300 N) was performed to determine compressive stiffness. Foraminal height and segmental lordosis were evaluated using $\mathrm{x}$-ray image analysis following controlled posterior rod compres- sion $(170 \mathrm{~N}) .4$ conditions were tested in random order: 1) intact; 2) expandable spacer(ES+PSR); 3) fixed ovoid spacer(FOS+PSR); and, 4) fixed rectangular spacer(FRS+PSR). Data were analyzed using RM-ANOVA $(\mathrm{P}<0.05)$.

Results: All constructs demonstrated greater stability than intact $(p<0.001)$. No significant differences existed between constructs in either ROM (F/E, AR, LB) or compressive stiffness testing $(p>0.75)$. ES+PSR demonstrated significantly increased mean foraminal height at L4-5 than FRS+PSR ( $p=0.026)$. ES+PSR demonstrated higher anterior disc height than FOS+PSR $(\mathrm{p}=0.04)$, and higher posterior disc height than intact $(\mathrm{p}=0.002)$, FOS $+P S R$ $(p<0.001)$, and FRS+PSR $(p<0.001)$. There were no significant differences in segmental lordosis between FOS+PSR $\left(10.0 \pm 2.3^{\circ}\right)$, ES+PSR $\left(7.9 \pm 0.3^{\circ}\right)$, and FRS+PSR $\left(10.1 \pm 1.3^{\circ}\right)$.

Conclusion: The expandable interbody spacer provided similar segmental lordosis compared to traditional spacers of different shapes, with the benefit of increased foraminal decompression and greater disc height compared to non-expandable spacers. All factors must be considered when tailoring the choice of interbody spacer for TLIF approach to match surgical goals and indications.

360 Interleukin-13 Targeting for the Treatment of Malignant Peripheral Nerve Sheath Tumors

Oliver Daniel Mrowczynski, MS; Russell Payne, MD; A. B. Madhankumar; Alexandre Bourcier, BS; Christine Y Mau, MD; Vagisha Ravi; Becky Slagle-Webb; Kimberly Harbaugh, MD; Elias Boulos Rizk, MD; James R. Connor

Introduction: Malignant peripheral nerve sheath tumors (MPNSTs) are soft tissue sarcomas that arise from peripheral nerves. A target of MPNSTs is the receptor for interleukin-13 (IL-13R). IL-13R has an a1 and a2 subtype, and activation of a1 leads to apoptosis. MPNSTs have increased expression of the a2 subtype, IL-13Ra2. $\mathrm{IL}-13 \mathrm{Ra} 2$ is an oncogene that acts as a decoy receptor which has a higher affinity for IL-13, allowing cancer to evade death by binding and sequestering all of the IL-13 thus inhibiting al activation. MPNSTs are currently treated with surgical resection, sometimes requiring complete limb amputation, as well as chemoradiation, all of which demonstrate limited effectiveness, and highlight the necessity for novel therapies. The goal of this study was to demonstrate the effectiveness of intratumoral IL-13 targeted pseudomonas exotoxin (GB-13) and IL-13 targeted liposomal doxorubicin (IL13-Lip-Dox) for MPNST treatment. The upregulated IL-13Ra2 on MPNSTs provides a unique opportunity for utilizing these therapies to precisely target MPNSTs and cause tumor cell death.

Methods: We interrogate the effectiveness of GB-13 and IL13-Lip-Dox to cause MPNST apoptosis in vitro. Furthermore, we perform IL13-Lip-Dox and GB-13 treatment in an orthotopic MPNST murine model. We perform molecular analysis on the tumors with $\mathrm{H} \& \mathrm{E}$, and immunohistochemistry of Ki67 and Cleaved caspase 3 .

Results: GB-13 and IL13-Lip-Dox treatment caused significant MPNST cell death in vitro. Both treatments led to a significant decrease in tumor progression in vivo. Immunohistochemical analysis showed increased necrosis, decreased Ki67, and increased cleaved caspase-3. The specific targeting of IL-13Ra2 also lead to a decreased side effect profile due to IL-13Ra2 not being expressed in normal tissues.

Conclusion: The current MPNST treatment paradigm is composed of 3-prongs: surgery, chemotherapy, and radiation, all of which have been demonstrated to be unsatisfactory. This lays the groundwork for the change of this paradigm and subsequently optimal patient outcomes by the addition of a 4th prong, intratumoral treatment with GB-13 as well as enhancing the chemotherapeutic arm with targeted liposomal therapy. 


\section{MLN8237 Treatment in an Orthoxenograft Murine Model for Malignant Peripheral Nerve Sheath Tumors}

Christine Y Mau, MD; Russell Payne, MD; Oliver Daniel Mrowczynski, MS; Becky Slagle-Webb; Dawit Aregawi, MD; A. B. Madhankumar; Kimberly Harbaugh, MD; James R. Connor; Elias Boulos Rizk, MD

Introduction: Malignant peripheral nerve sheath tumors (MPNSTs) have increased expression of the oncogene aurora kinase A (AURKA) leading to enhanced cellular proliferation, which makes them extremely aggressive. MPNSTs are treated with surgical resection and chemoradiation or chemotherapy with doxorubicin/ifosfamide. MLN8237 is an orally bioavailable AURKA inhibitor that may be effective in treatment of MPNSTs.

Methods: Cytotoxicity analysis was performed on a MPNST cell line (STS26T) to assess the efficacy of MLN8237 in vitro. A murine orthoxenograft MPNST model transfected to express luciferase was developed in order to assess the efficacy of MLN8237 in the treatment of MPNSTs in vivo. Mice with confirmed tumor on in vivo imaging were divided into three separate groups: 1) Control, 2)MLN8237, 3)doxorubicin/ifosfamide. Treatment was carried out for 32 days with weekly imaging until post-injection day 42. Average bioluminescence was compared using a one-way ANOVA. Survival analysis was performed using Kaplan-Meier curves.

Results: Immunohistochemistry showed robust expression of AURKA in tumors cells. Cytotoxicity analysis revealed STS26T susceptibility to MLN8237 in vitro. The group receiving MLN8237 treatment showed a statistically significant difference in tumor size starting at post-injection day 21 persisting through the end of the study. This group also showed decreased tumor size compared to the doxorubicin/ifosfamide group at the conclusion of the study $(\mathrm{p}=0.036)$. Survival analysis revealed a significantly increased median survival in the MLN8237 group ( 83 days) compared to both the control (64 days) and doxorubicin/ifosfamide (67 days). A hazard ratio comparing the treatment groups showed a decreased hazard rate in the MLN8237 group compared to the doxorubicin/ifosfamide groups $(\mathrm{HR}=2.945, \mathrm{p}=0.0134)$.

Conclusion: The results of our study demonstrate MLN8237 is superior to doxorubicin/ifosfamide treatment in an orthoxenograft murine model. This data has major implications on the future of MPNST research by providing a robust murine model as well providing evidence that MLN8237 may be an effective treatment for MPNSTs.

\section{Outcomes Following Surgery for Peroneal Intraneural Ganglion Cysts} MD

Thomas J. Wilson, MD; Michael G. Rock; Robert J. Spinner,

Introduction: Patients with peroneal intraneural ganglion cysts often present with dense neurologic deficits attributable to peroneal nerve dysfunction. When discussing surgery, we are often asked a typical set of questions by the patients, including: What is the likely neurologic outcome? What is the likelihood of recurrence? The objective of the current study was to utilize our large surgical experience to answer the typical questions that arise from patients when discussing peroneal intraneural ganglion cysts preoperatively.

Methods: Retrospective analysis of all patients who underwent surgery for a peroneal intraneural ganglion cyst between January 1, 2000 and April 1, 2017 was performed. Postoperative neurologic outcomes and radiologic recurrences are reported.
Results: The cohort consisted of 65 patients. Average clinical follow-up was 14 months. Median dorsiflexion preoperatively was $2 / 5$. Median eversion preoperatively was $4 / 5$. Median dorsiflexion at last follow-up postoperatively was $5 / 5$. Median eversion at last follow-up postoperatively was $5 / 5$. Among those patients with at least 6 months of follow-up, 48/51 (94\%) patients had a good outcome (at least MRC 4-/5 dorsiflexion). Average time to achieve MRC 4-/5 or better dorsiflexion was 7 months. Average time to documented maximal recovery of dorsiflexion was 9 months. No patients had new or worsened motor deficits postoperatively. Postoperatively, 6 (9\%) patients developed a radiologic recurrence of the cyst, all extraneural, with $4(6 \%)$ undergoing a repeat operation for the recurrence. Median time to recurrence was 15 months, with a range of 6134 months.

Conclusion: The data from our large series support excellent postoperative motor outcomes, despite the fact that patients often present with dense weakness of peroneal-innervated musculature. The surgical approach eliminates risk of intraneural recurrence and minimizes risk of extraneural recurrence.

363 Recovery Period Following Nerve Transfer and Nerve Grafting in Traumatic Adult Brachial Plexus Injuries

\section{Mohamed E. Elsbaey; Ayman E. Galhom}

Introduction: The clinical management of the brachial plexus injuries is very challenging when the patient is seeking for restoring the full motor power, gaining the full recovery and returning back into the usual lifestyle. Through different approaches and techniques, the nerve transfer and nerve grafting can be applied to restore the main motor movements like shoulder abduction, elbow flexion and hand grip.

Methods: Descriptive clinical case study , Between January 2013 and April 2016, the brachial plexus team operated 12 patients who had traumatic brachial plexus injuries. Nerve transfer and nerve grafting techniques were used in those patients. Follow up was done by clinical evaluation each 3 months using the Medical Reasearch Council (MRC) scale for assessment of the motor power.

Results: 12 male patients were operated. Nerve transfer was done in 7 patients while nerve grafting was done in 5 patients. The age was ranged from 20 years to 42 years with a mean of 31 years. 9 patients were presented with upper brachial plexus injuries, 2 patients with pan brachial plexus palsy and 1 with lower brachial plexus injury. 9 cases gained functional recovery, 1 case improved without functional recovery, 1 case did not show any improvement uptill now, while 1 case was died 4 months postoperative. Average of recovery period in patients underwent nerve grafting technique was 19 months while was 8 months in patients who underwent nerve transfer.

Conclusion: Nerve grafting and nerve transfer are effective techniques in management of the brachial plexus injuries, but the recovery period in nerve transfer is much less than that in nerve grafting.

364 A 10-Year Analysis of Peripheral Nerve Injuries in High School Sports

Scott L. Zuckerman, MD; Zack Kerr; Lauren Pierpoint; Khoi Duc Than, MD; Thomas J. Wilson, MD

Introduction: Sports surveillance databases provide valuable information regarding common ailments, yet little is known about rare peripheral nerve injuries. Our objective was to characterize peripheral nerve injuries in high school athletics with respect to incidence, sport, mechanism, time loss, and diagnoses. 
Methods: Sport-related nerve injury data on high school athletes were collected during the 2005-2006 through 2015-2016 academic years via the High School Reporting Information Online (RIO) database. All injuries were reported by certified athletic trainers (ATs). Descriptive statistics were performed.

Results: A total of 588 peripheral nerve injuries were recorded during the 2005/06-2015/16 academic years, with an overall incidence of 1.46/100,000 athletic exposures (AE; 95\% CI 1.34$1.58)$. Boys' football had the majority of injuries $(71.3 \%)$ and the highest injury rate $(5.46 / 100,000 \mathrm{AE}, 95 \% \mathrm{CI} 4.93-5.98)$, followed by boys' wrestling (7.1\%) and boys' baseball (3.4\%). Over half $(50.3 \%)$ of peripheral nerve injuries resulted in time loss $<1$ week, while $9.4 \%$ were season-ending injuries. The most common mechanisms were player contact $(67.3 \%)$ overuse $(10.0 \%)$, and surface contact $(9.7 \%)$. A specific diagnosis was available for $40(6.8 \%)$ injuries, including upper extremity stinger $(65.0 \%)$, spinal cord neurapraxia $(7.5 \%)$, subacromial nerve impingement $(5.0 \%)$, neuroma $(5.0 \%)$, axillary nerve palsy $(2.5 \%)$, sciatic nerve impingement $(2.5 \%)$, femoral nerve impingement $(2.5 \%)$, tarsal tunnel syndrome $(2.5 \%)$, peroneal neuropathy $(2.5 \%)$, thoracic outlet syndrome $(2.5 \%)$, and ulnar nerve subluxation $(2.5 \%)$.

Conclusion: Peripheral nerve injuries are rare among high school athletes, occurring most commonly in boys' football. While most are minor, approximately 1:11 were season-ending. Upper extremity stingers were most common, though specific diagnoses were available for only $10 \%$ of injuries. Many diagnoses were associated with overuse injuries (tarsal tunnel syndrome, ulnar nerve subluxation) rather than acute, traumatic events. Working with ATs, more specific diagnostic information is needed in the RIO database to allow better understanding of peripheral nerve injuries incurred.

365 Targeting Inflammatory Response in Injured Neural Tissue With Novel Localized Polymer-based Steroid Delivery

Timur Urakov, MD; Susana Cerqueira; Jessica Johnson; Tianyu Zhao; Mary Bunge; Rajiv Saigal, MD, PhD

Introduction: Local delivery of steroids to the spinal cord injury (SCI) site may be a favorable alternative to systemic delivery in order to reduce side effects. Previous methods described indwelling catheters and intraparenchymal implantation as mechanisms for local delivery. We developed a polymer-based drug delivery system for local, controlled release of dexamethasone over several days, producing steroid coverage during the critical time after injury.

Methods: Fluorescein-conjugated dexamethasone was incorporated into polyvinylpyrrolidinone (PVP), and gel droplets were formed by UV polymerization. Chemical modifications were made to optimize for drug release over 72 hours. To evaluate the drug release profile, fluorescence levels were quantified from media containing dexamethasone-PVP gels. Cell toxicity and response to an inflammatory stimulus were studied in Schwann cells and microglia Metabolic activity was measured using the MTS assay. Microglia were isolated from rat pup cortices and stimulated with bacterial lipopolysaccharides (LPS) +/- interferon-gamma (IFN) to model an inflammatory environment in vitro. Production of nitric oxide (NO) was measured with Griess reagent. TNFa and reactive oxygen species (ROS) were also measured using ELISA and an enzymatic conversion assay. Luminex was used for high throughput evaluation of multiple inflammatory markers.

Results: Polymer gels released dexamethasone over 3 days. Addition of LPS +/- IFN to microglia induced a significant increase in the production of $\mathrm{NO}$, which was significantly decreased after addition of the dexamethasone alone as well as PVP. A similar trend was observed in reduction of TNFa and ROS production. Luminex showed decrease in multiple inflammatory markers in response to the polymer-based treatment.

Conclusion: Dexamethasone was successfully incorporated into the polymer with sustained drug delivery profile. The results suggest safety and efficacy in an in vitro inflammation model. Future directions include translation experiments in animal models of SCI.

366 Management of Chronic Pain Post Neurectomy: A Role for Dorsal Root Ganglion Stimulation

\section{Line G. Jacques, MD, MSc, FRCS(C), DABNS}

Introduction: Chronic neuropathic pain may be iatrogenic or post traumatic and can be a disabling and crippling in some individuals. Patients may have significant sleep disturbances, experience socio-psychological changes, along with significant physical limitations. A combination of pharmacological treatments along with physical therapy and local infiltrations may be useful. In certain cases, surgical approaches including selective neurectomy can be effective; others will remain chronic and intractable despite all interventional measures. Neurostimulation techniques that can include: spinal cord stimulation, peripheral nerve stimulation and most recently dorsal root ganglion stimulation have shown promising results in the treatment of chronic neuropathic pain

Methods: Pre-operatively, patients were screened for suitability for dorsal root ganglion stimulation after triple neurectomy for groin pain, superficial peroneal nerve neurectomy for ankle and foot pain, saphenous neurectomy for knee pain. Quality of life scores, VAS, and paresthesia mapping were recorded as baseline parameters as well as post operatively. Up to 4 percutaneous leads were placed epidurally at the dorsal root ganglion.

Results: Overall, quality of life measures were improved by greater than $50 \%$.The VAS scores were reduced by $50 \%$ as well. There was one case of lead migration and no infections reported. The follow up was on average of 10 months .

Conclusion: Dorsal root ganglion stimulation is a viable option in the treatment of chronic neuropathic pain and should be considered as part of the pain treatment algorithm when treating this challenging patient population

367 Sustained Interleukin-10 Delivery Reduces Inflammation and Improves Motor Function After Spinal Cord Injury

Dan Hellenbrand; Amgad S. Hanna, MD, FAANS; Mallory Filipp; Benjamin Travis

Introduction: The anti-inflammatory cytokine interleukin-10 (IL-10) has been explored previously as a treatment method for treatment of spinal cord injury (SCI) due to its ability to attenuate pro-inflammatory cytokines and reduce apoptosis. Primary limitations when using IL-10 are that it is rapidly cleared from the injury site and that it does not cross the blood spinal cord barrier. Here, mineral-coated microparticles (MCMs) were used to obtain a local sustained delivery of IL-10 directly into the injury site.

Methods: Female Sprague-Dawley rats were contused at T10 and treated with an intraperitoneal injection of IL-10 or with an intramedullary injection of IL-10 or MCMs bound with IL-10 (MCMs+IL-10). After treatment, cytokine levels were measured in the spinal cord, functional testing and electrophysiology were performed, axon tracers were injected into the brainstem and motor cortex, macrophage levels were counted, and lesion size was measured.

Results: When treated with IL-10+MCMs, IL-10 was significantly elevated in the injury site, inflammatory cytokines were 
significantly suppressed, prompting a larger ratio of M2 to M1 antigen expressing macrophages. Significantly more axons were preserved within the rubrospinal and reticulospinal tracts through the injury site when treated with MCMs+IL-10; however, there was no significant difference in corticospinal tract axons preserved, regardless of treatment group. The rats treated with MCMs+IL-10 were the only group with a significantly higher functional score compared to injured controls 28 days post-contusion.

Conclusion: These data demonstrate that MCMs can effectively deliver IL-10 for an extended period of time and sustained IL-10 reduces pro-inflammatory cytokines, which improves functional recovery after SCI.

368 Pro-regenerative Macrophages Regulate Schwann Cell Dynamics Following Nerve Injury

Rajiv Midha, MD

Introduction: Many individuals are affected by nerve injury, resulting in functional deficits and pain. Pro-regenerative macrophages are well known for their role in promoting tissue repair and remodeling a process that is important for full functional recovery in many injury environments. However, the role of pro-regenerative macrophages in nerve injury is not as well defined. We aimed to decipher the role macrophage during nerve regeneration.

Methods: First, we performed RNAseq on macrophages from day 3 and 8 post-injury, discovering that these cells expressed an array of array of factors including Growth Arrest Specific 6 (Gas6), HBEGF and IL6. Following the addition of these factors to immature Schwann cell cultures, we found that Schwann cells were responsive to Gas6 and IL6, namely Schwann cells exited cell cycle, suggestive of a role for macrophages during the process of Schwann cell maturation. We performed nerve injury in Thy1-GFP reporter rats, and eight days later treated them with mannosylated clodronate liposomes to ablate macrophages, or control liposomes.

Results: Using compound muscle action potential analysis, we found that macrophage ablation resulted in long-term deficits in conduction velocity, suggesting poorer myelination, but no effect of conduction amplitude. In addition, we found that there was an increase in Schwann cell density, specifically immature Schwann cells, as well as a decrease in the percentage of remyelinated axons in the ablation group. Finally, macrophage targeted knock out of Gas6 in nerve injury results in an increase in Schwann cell density as well as a reduction on remyelinated internode length demonstrating that macrophage-derived Gas6 independently regulates Schwann cell dynamics in peripheral nerve injury.

Conclusion: Taken together, this dataset has enhanced our understanding of the mechanisms driving recovery following nerve injury an important step towards developing more targeted treatment options.

369 Guidelines for the Use of Electrophysiological Monitoring For Surgery of the Human Spinal Column and Spinal Cord

Christopher D. Shank; Mark N. Hadley, MD, FACS; Curtis J. Rozzelle, MD; Beverly C. Walters, MD, MSc, FRCS(C), FACS

Introduction: Electrophysiological monitoring techniques allow assessment of spinal cord sensory pathways, motor pathways, and spinal root function. Recording of these parameters after spinal cord injury or during spinal column or spinal cord operative procedures (intraoperative monitoring) has the potential to evaluate the integrity of the sensory and motor pathways and spinal roots in real-time fashion. Theoretically, alterations in established recordings during spinal cord/spinal column surgery implicate an impending spinal cord injury -- an injury that could potentially be prevented to help assure a better outcome following surgery.

Methods: A National Library of Medicine (PubMed) computerized literature search from 1966 to 2016 was undertaken, revealing 82 articles on the diagnostic and therapeutic effect of intraoperative monitoring. Using standard guidelines methodology, articles were assigned classes of evidence (I, II, or III.) The evidence was then synthesized into recommendations of Level I, III, and III, corresponding to the designated classes of evidence.

Results: Intraoperative electrophysiological monitoring recording during spinal surgery is a reliable and valid diagnostic adjunct to assess spinal cord integrity and is recommended if utilized for this purpose. Motor evoked potential recordings are superior to SSEP recordings during spinal surgery as diagnostic adjuncts. Electrophysiological monitoring, including SSEP and MEP recordings, during spinal surgery has no therapeutic relationship with neurological outcome and is not recommended for this purpose. There is no evidence that the expense of IOM and its interpretation during spinal column/spinal cord surgery justifies its use in attempting to prevent iatrogenic spinal cord injury.

Conclusion: The use of intraoperative monitoring, while an effective diagnostic adjunct, does not appear to alter patient outcomes following spinal column or spinal cord surgery. In light of the existing evidence, intraoperative monitoring should NOT be considered standard of care.

370 Persistent Hyperexcitability of Human Primary Sensory Neurons after Spinal Cord Injury

Robert Y North, MD; Yan. Li, PhD; Tejaswi Marri, BS; Haley Rhodes; Ganesh Rao, MD; Laurence D. Rhines, MD; Claudio Esteves Tatsui, MD; Patrick M Dougherty

Introduction: Neuropathic pain develops in approximately $40 \%$ of patients following spinal cord injury (SCI). Classically, central nervous system mechanisms have been implicated as the primary driving force for this chronic pain. However, recent studies using animal models of SCI have revealed that primary sensory neuronal hyperexcitability may also be an important contributor. Whether this pathophysiology occurs for human primary sensory neurons after SCI is not established.

Methods: Human dorsal root ganglia were obtained from patients undergoing oncological spine surgery that necessitated sacrifice of spinal nerve roots as part of standard of care. Clinical data was obtained through retrospective review or collected at time of study enrollment. Neurons were dissociated from surrounding tissue, briefly maintained in cell-culture (24-72 hours), and examined with whole-cell patch clamp techniques. Electrophysiological measures of excitability were compared for primary sensory neurons from patients with and without prior SCI. In order to minimize confounding by peripheral nerve pathology, neurons from dorsal root ganglia with nerve root compression or from patients with either neuropathic pain or peripheral neuropathy were excluded.

Results: Electrophysiological recordings from 26 dissociated human sensory neurons were obtained from 5 patients. 8 neurons were from a single patient with prior T10 ASIA D SCI ( $>6$ months prior); 18 neurons from patients without any prior history of SCI. Compared to neurons from patients without SCI, human sensory neurons from the patient with SCI were significantly more excitable (Step rheobase: $250 \pm 50$ vs $1140 \pm 200$ pA p $<0.001$, Threshold potential: $-15.5 \pm 2.5$ vs $4.7 \pm 3.4 \mathrm{mV}$ p $<0.05$ ).

Conclusion: We provide the first evidence for persistent hyperexcitability in human primary sensory neurons after SCI. These findings support the hypothesis that the peripheral nervous system, in addition to the central nervous system, may be relevant to the development of SCI related neuropathic pain. 
371 Role of the Sodium/Glutamate Blocker Riluzole in Enhancing Functional Outcomes in Patient Undergoing Surgery for Degenerative Cervical Myelopathy: Results of the Prospective, Multicentre Double Blind Controlled CSMProtect Randomized Controlled Trial

Michael G. Fehlings, MD, PhD, FRCS(C), FACS; Branko Kopjar, MD; Henry Ahn; Francis Farhadi, MD, PhD; Christopher I. Shaffrey, MD, FACS; Ahmad Nassr, MD; Praveen V. Mummaneni, MD; Paul M. Arnold MD; Bradley Jacobs, MD; K Daniel Riew, MD; Darrel S. Brodke, MD; Alexa

Introduction: Degenerative cervical myelopathy (DCM), which encompasses cervical spondylotic myelopathy and ossification of the posterior longitudinal ligament, is the most common cause of spinal cord impairment. Decompressive surgery is the most effective treatment, however, most patients are left with residual neurological impairment and some experience neurological decline. Based on strong preclinical basic science evidence and collateral evidence from trials in human spinal cord injury and amyotrophic lateral sclerosis, we sought to explore if the sodiumglutamate antagonist riluzole would enhance neurological recovery and reduce perioperative neurological decline.

Methods: This is a phase III multi-center, double-blind, placebocontrolled, randomized controlled trial. Between March 2012 and June 2017, 300 surgically naive patients with moderate to severe DCM were enrolled at 16 sites. Subjects were randomized 1:1 to either the $50 \mathrm{mg}$ riluzole bid or placebo-controlled group, beginning the medications at 14 days pre-surgery and ending at 28 days postoperative. Follow-up was at 6 - and 12-months to determine the primary endpoint, change in mJOA scores; and the secondary endpoints, change in SF-36v2, Neck Disability Index (NDI), Nurick grade, EQ-5D, ASIA motor and sensory scores, Bazaz scale, Visual Analog Scale (VAS) for Pain, grip strength and neurological compilations.

Results: The average age was 57.9 years (SD 10.2); $55.7 \%$ males; $80.1 \%$ white; $9.7 \%$ black. Baseline mJOA score was 11.84 (SD 1.5, range 8 to 14); Nurick grade was 3.3 (SD 0.8); NDI 42.9 (SD 13.5); VAS arm/shoulder 4.7 (SD 2.9); VAS neck 4.9 (SD 3.0). The study will complete in December 2017 with a projected follow-up rate exceeding $90 \%$. Efficacy results will be available and presented at the conference.

Conclusion: This study contributes Level I evidence concerning efficacy and safety of riluzole as an adjunct therapy to decompressive surgery for patients with DCM.

372 Potential of Human NP-like Cells Derived From Umbilical Cord to Treat Degenerative Disc Disease: Novel Mechanism for Disk Regeneration

Mick J. Perez-Cruet, MD, MS; Esam A Elkhatib, MD, PhD; Naimisha Reddy Beeravolu, MS; Irfan Khan; Christina Marie McKee, MS; Rasul Chaudhry, PhD

Introduction: Degenerative disc disease (DDD) is a common spinal disorder that manifests with lower back pain. The degeneration of intervertebral disc (IVD) is characterized by the loss of extracellular matrix and dehydration of the nucleus pulposus (NP) of IVDs. Currently, there is no biological treatment to cure this debilitating ailment.

Methods: In this study, we investigated the efficacy of NP-like cells (NPCs) derived from the umbilical cord (UC) MSCs in restoring degenerated IVDs using a rabbit DDD model. UC -MSCs were induced to differentiate into NPCs by using differentiation medium (DM) for two weeks, labeled with PKH26 and then injected into the degenerated IVDs.
Results: Eight weeks posttransplantation analysis showed that structure and cellularity of the NP improved only in the IVDs that received NPCs. Transplanted IVDs also had higher sGAG and water content compared to the sham and degenerated IVDs. The transplanted cells survived, integrated, and dispersed in the damaged areas of the NP and were functionally active as they expressed human genes, SOX9, ACAN, COL2, FOXF1, KRT19, PAX6, CA12 and COMP as well as human proteins, SOX9, ACAN, COL2 and FOXF1 implicated in NP biosynthesis.

Conclusion: These results suggest that NPCs were capable of homing to regenerate NP. The molecular mechanism for NP regeneration was proposed to be regulated via the TGFß1 pathway. This study for the first time demonstrates the feasibility and efficacy of human NPCs derived from UCMSCs to regenerate NP in a rabbit model. These findings should spur interest for clinical studies to treat DDD using NPCs.

373 Novel Device to Achieve Intervertebral Disc Regeneration Using Human Stem Cell Generated Nucleus Pulposus Cells

Mick J. Perez-Cruet, MD, MS; Esam A Elkhatib, MD, PhD; John Pepper; Naimisha Reddy Beeravolu, MS; Christina Marie McKee, MS; Rasul Chaudhry, PhD

Introduction: Intervertebral disc (IVD) degeneration is characterized by the loss of nucleus pulposus (NP) leading to disc space collapse which is a common cause of low back pain. Currently, degenerative disc disease (DDD) has no biological cure and human stem cell therapy is increasingly being considered for its treatment. However, stem cells alone may not be adequate to provide bio-mechanical disc height restoration. Disc restorative technology is being investigated that might restore the bio-mechanical function of the degenerated disc via a process of gradual tissue growth generated distraction (TGGD). Once disc height is restored the injection of nucleus pulposus cells (NPCs) can restore the biological function of the degenerated disc.

Methods: In this study, we investigated the feasibility of human umbilical cord mesenchymal stem cells (UC-MSCs) differentiated into NPCs invitro in an in-vivo rabbit model of DDD. Histological, biochemical and gene expression analysis was preformed to determine the optimal cell for NP regeneration (Fig.3). Further finite element analysis was preformed of a mechanical technology called Annulo (MI4Spine LLC, Bloomfield, MI) used to achieve disc height restoration.

Results: Transplanted NPCs survived, integrated and displayed homing into nucleus pulposus (NP) of the rabbit degenerated disc with a significant improvement in the nucleus pulposus histology, cellularity, extracellular matrix proteins, water, and glycosaminoglycan contents. In addition, the IVDs receiving NPCs had higher expression of nucleus pulposus specific human markers SOX9, ACAN, COL2, FOXF1, and KRT19. Finite element analysis of Annulo device revealed the optimal bio-mechanical forces needed to gradually restore disc height like Ilizarov.

Conclusion: NPCs were efficient in regenerating the degenerated intervertebral disc and provide basis and impetus for clinical studies to treat DDD. A combination of TGGD technology can restore disc height first followed by injection of NPCs to restore the nucleus pulposus and may result in a viable biological treatment for patients suffering from DDD.

374 The Use of Magnetic Resonance Neurography and Fiber Tracking in Peripheral Nerve Sheath Tumors

Megan M Jack, MD, PhD; Kushal Shah; Brian Everist, MD; Phillip D Hylton, MD 
Introduction: Magnetic Resonance neurography or peripheral neurography is a technique which uses diffusion to differentiate between intraneural and perineural tissues. Diffusion tensor imaging (DTI) technology has only recently been applied in the peripheral nervous system due to challenging technical factors and lack of widespread availability. It allows for fascicle patterns to be visualized particularly in the setting of peripheral nerve sheath tumors. This technique helps determine the anatomic location of these nerve fibers in relation to the mass, which is particularly helpful at distinguishing neuromas from schwannomas. This technology has been underutilized due to the challenges of requiring complex software to produce fiber tracks and the inability to translate these images into the operating room. BrainLab iPlan FiberTracking overcomes the reliance on highly specialized software and need for extensive training.

Methods: BrainLab iPlan FiberTracking software was used to outline a peripheral nerve sheath tumor and integrate raw DICOM DTI data to produce nerve fiber tracts of the radial nerve in relation to the tumor in an automated fashion.

Results: Here, we demonstrate a proof of concept utilizing BrainLab iPlan FiberTracking software that is commonly available and utilized in surgical suites. This software was used to produce a 3-D reconstruction of the radial nerve fiber tracts with an associated peripheral nerve sheath tumor. Similarly, other cases of peripheral nerve sheath tumors have been successfully tracked.

Conclusion: While the software is commonly used in the central nervous system, it has not been reported to have been used in the peripheral nervous system. This software offers a high usability and produces anatomically correct and reliable fiber tracts. This data offers clinicians an option to investigate peripheral nerve fibers in various pathologic states, to plan appropriate operative trajectories to peripheral nerve pathology, and to improve surgical outcomes for patients with peripheral nerve sheath tumors.

375 One Stage Posterior Minimal Laminectomy and Video-Assisted Thoracoscopic Surgery (VATS) for Removal of Thoracic Dumbbell Tumor

\section{Kyoung-Hyup Nam; Jun Seok Lee, M.D.; Dong Wuk Son}

Introduction: This study was conducted to assess the surgical results of one-stage posterior minimal laminectomy and videoassisted thoracoscopic surgery (VATS) for the treatment of thoracic dumbbell tumor and to describe its precise technique. In addition, we investigated the technique's usefulness and limitations.

Methods: Seven cases of thoracic dumbbell tumor (two men and five women, mean age, 43 years) were analyzed retrospectively. Pathological findings included schwannoma in three patients, neurofibroma in three patients, and hemangioma in one patient. The location of tumors varied from T2/3 to T12/L1. Dumbbell tumors were resected by one-stage operation using posterior laminectomy followed by VATS without instrumentation. Clinical data were reviewed.

Results: The mean follow-up period was 25 months (range, 358 moths), and the operative time ranged from 255 to $385 \mathrm{~min}$ (mean, $331 \mathrm{~min}$ ), with estimated blood loss ranging from 110 to $930 \mathrm{ml}$ (mean, $348 \mathrm{ml}$ ). The tumor was completely resected without instrumentation and postoperative instability in all cases. Postoperative complications included atelectasis and facial anhydrosis in one case each.

Conclusion: One-stage posterior minimal laminectomy and VATS may be a safe and less invasive technique for removal of thoracic dumbbell tumor without instability. This method has the advantage of early ambulation and rapid recovery because it reduces blood loss and postoperative pain.
376 Relationship Between Opioid Use and Outcomes in Patients Undergoing Basivertebral Nerve Ablation for Chronic Low Back Pain

Alfred L. Rhyne, MD; Rick Sasso, MD; Jeffrey S. Fischgrund, MD; Alpesh Patel; Wellington Hsu, MD; Av Edidin, Ph.D.; Peter Vajkoczy, MD

Introduction: Opioid dependency is a social problem reaching near epidemic proportions in the U.S., with over 20,000 prescription opioid related overdoses observed in 2015. Opioids may be used to manage pain in certain patients diagnosed with chronic low back pain (CLBP); ideally, alleviation of CLBP may affect consumption of opioid pain relievers.

Methods: Opioid use was monitored in a randomized clinical trial (SMART) to investigate the effect of basivertebral nerve (BVN) ablation on CLBP. The primary outcome variable in the trial was the observed change in ODI at 3 months; a statistically significant decrease in ODI was observed in the treatment group. Opioid medication use history was collected at each follow-up interval and subsequently converted to opioid equi-analgesic weekly dose; at each follow-up time point individual use was categorized as unchanged, increased, or decreased. After the conclusion of the study, we compared the average change in ODI and VAS in the treatment group patients who increased as opposed to decreased use of opioid medication at 3 months

Results: Results: Short acting opioids were used at time of enrollment by 57 out of 145 patients on an intent-to-treat (ITT) basis and 48 out of 127 patients on a per-protocol (PP) basis. At the three month primary endpoint, the mean improvement in ODI and VAS in patients increasing and decreasing their use of opioids was compared. These metrics were statistically and measurably inferior in patients reporting an increased use of opioids as shown in Table 1.

Conclusion: Patients who increased their consumption of opioid medications following RF ablation of the BVN for relief of CLBP had poorer outcomes measured using ODI and less pain relief measured using VAS than did patients with constant or decreasing use of opioids. These data suggest that there exists a relationship between decreased functional and pain outcomes and increased use of opioid pain relievers in patients with CLBP, and that relieving chronic back pain may be a factor in reducing opioid usage.

377 Perineural Spread of Melanoma to the Brachial Plexus: Identifying the Anatomic Pathway(s)

Tomas Marek, MD; Ruple S Laughlin, MD; Benjamin M. Howe; Robert J. Spinner, MD

Introduction: Perineural spread of melanoma is a wellknown mechanism of metastasis in cases involving cranial nerves. Brachial plexus involvement is rare and the pathway is unknown.

Methods: A retrospective review of the Mayo Clinic database was performed to identify patients with a history of melanoma and brachial plexus compromise between 1994 and 2017. Inclusion criteria included a history of melanoma, brachial plexopathy, radiological features consistent with perineural spread and biopsy of melanoma within nerve.

Results: We identified 42 cases, 24 men and 18 women with a median age of 61 years (37 84 years) with a brachial plexopathy and a history of melanoma. After review of their clinical information, 2 cases fulfilled inclusion criteria. In 40 excluded cases, brachial plexopathy was caused by: metastasis to axillary lymph nodes $(\mathrm{n}=11)$; trauma $(\mathrm{n}=8)$; post-surgical sequelae $(\mathrm{n}=7)$; 
tumors other than melanoma $(n=5)$; inflammation $(n=5)$; radiation $(\mathrm{n}=2)$; a combination of radiation and post-surgical changes $(\mathrm{n}=1)$; and radiculopathy $(\mathrm{n}=1)$.

Conclusion: The 2 identified cases both showed similar clinical and radiological features. We believe that there is a pattern of perineural spread to the brachial plexus through the cervical plexus. Literature review shows several recently published cases demonstrating analogous mechanism of melanoma spread involving upper cervical nerves which supports our proposed pathway.

378 Comparison of Human and Rat Neural Stem/ Progenitor Cell proliferation and Differentiation: Implications for the Successful Translation of Rodent Stem Cell Therapies to Humans

Eve C. Tsai, MD, PhD, FRCS(C), CIP; Ahmad Galuta, BS; Diana Cristina Ghinda, MD; Carolyn Lai, MD; Mohammed Alsharden, MBBS; Mohammed Y AlGhamdi, MD; Mahmoud Bedaiwy; Suzan Chen, MD

Introduction: While several promising neural stem/progenitor cell (NSPC) therapies for spinal cord injury have been developed in animal models, therapeutic translation to humans has often failed. While the direct comparison of human and rodent NSPCs to assess intrinsic cell differences may improve the translation, this comparison using identical culture conditions has not been reported previously due to the difficulty in obtaining viable human spinal cord tissue to allow the culture of NSPCs.

Methods: Regional ethics board approval was obtained. To compare the intrinsic proliferative and differentiation potential of human and rat spinal cord NSPC, we obtained viable human spinal cord tissue from organ donors $(n=10)$ and Sprague Dawley rats $(n=6)$ and isolated NSPCs from this tissue. Using identical cell culture conditions, human and rat NSPCs were assessed for proliferation in media containing epidermal growth factor and fibroblast growth factor -2 . Proliferation was assessed with BrdU, Sox $2+$ and DAPI labeled cell counts. Differentiation potential was assessed with $1 \%$ fetal bovine serum administration. Response to retinoic acid (RA), BMP4, or PDGF-AA to induce neural, astrocytic and oligodendrocytic fates, respectively, was assessed.

Results: Rat NSPC proliferation rate was twice $(2.3 \pm 0.8)$ that of humans. Rat NSPCs differentiated more into astrocytes $(71.8 \pm 5.6 \%)$ compared to neurons $(15.2 \pm 4.2 \%)$ and oligodendrocytes $(2.82 \pm 1.3 \%)$. Human NSPCs differentiated more into neurons $(68.5 \pm 16.9 \%)$ with little $(<2 \%)$ gliogenesis. RA stimulated both human and rat NSPC differentiation into neurons, while PDFG only increased rat NSPC oligodendrocytic differentiation, and BMP4 only increased human NSPC astrocytic differentiation.

Conclusion: Due to the intrinsic differences between human and rodent NSPCs, translation of rodent NSPC therapy to humans may require longer duration of therapy because of the relative decrease in human NSPC proliferation rate, and exogenous growth factor administration may require modification given the differences in the intrinsic differentiation potentials and response to growth factors.

\section{Piriformis Syndrome After Lumbar Spinal Surgery}

\section{Masahiko Akiyama, MD, DMSc}

Introduction: Persistent or newly developed sciatica after successful decompression of lumbar lesion is not rare and known as failed back syndrome. Piriformis syndrome is one common cause of sciatica and could be one of the pathologies of failed back syndrome.

Methods: During March 2010 and June 2016, 157 lumbar spine surgeries were performed in our institution. Piriformis syndrome after lumbar spine surgery was defined as persistent or newly developed sciatica after surgery, sciatic notch tenderness and induced sciatica, and positive Freiberg sign. Fifteen cases were diagnosed as piriformis syndrome.

Results: Successful lumbar decompression or discectomy and absence of distal foraminal entrapment was confirmed with MR neurogram in all cases. No patients showed sciatic notch tenderness or positive Freiberg sign before surgery. Onset of sciatica was from 0 to 100 (ave. 20.9) days after surgery. Symptoms were subsided by pain medication alone in one patient, by combination of pain medication, stretching exercises, and injection of the piriformis syndrome trigger point in 13 patients. One patient required release of the piriformis tendon and sciatic neurolysis. A cause of piriformis syndrome in acute period may be coexistence of lumbar pathology and pelvic entrapment (double crush syndrome). One possible cause of this syndrome in chronic period could be muscle spasm due to change of gait posture.

Conclusion: Piriformis syndrome is not rare after lumbar spinal surgery and sometimes underestimated. In case of persistent or newly developed sciatica after successful decompression of lumbar pathology, diagnosis of piriformis syndrome should be considered.

380 Technical Feasibility and Safety of Ultrasound Guided Supraclavicular Nerve Block with Assistance of a Wearable Head-up Display

Jang Yoon, MD, MS; Tito G.M. Vivas-Buitrago, MD; Esther Kim; Robert E Chen; Alfredo Quinones-Hinoja, MD; Jose L DiazGomez, MD; Steven R. Clendenen, MD

Introduction: Since the introduction of the wearable head-up displays, there has been much interest in adopting these devices in clinical settings. Increasingly, more studies are being published in literature about their use to increase efficacy of workflow in the hospitals and clinics. In this study, we report the use of a novel video capture and streaming device that has capability to stream ultrasound images to a head-up display screen on Google Glass. This system was successfully utilized in supraclavicular nerve block.

Methods: We devised a novel video capture and streaming device that is capable of broadcasting videos via a password wireless network to a wearable head-up display. This system was connected to the ultrasound machine via High Definition Multimedia Interface (HDMI). Voice controlled video streaming software was installed on Google Glass.

Results: Ultrasound images were transmitted to Google Glass head-up display in real time without delays. The senior author (SRC) wore Google Glass to perform supraclavicular nerve block. He did not break the line of his sight towards the needle and did not divert his attention to look at the ultrasound monitor. The patient underwent the procedure without complications.

Conclusion: Head-up display allowed the clinician to monitor ultrasound images without stopping the procedure and turning his head to view the ultrasound screen. Head-up display has potential to enhance efficacy of ultrasound-guided procedures by eliminating the need to divert attention while performing critical procedural steps.

381 Voluntary Exercise Leads to Functional Recovery in Mice Following Peripheral Nerve Injury - A Role For Macrophage Polarization? $\mathrm{PhD}$

Megan M Jack, MD, PhD; Janelle Ryals; Douglas E Wright, 
Introduction: Peripheral nerve injury following trauma is often amenable to surgical intervention. Limited functional recovery remains a challenging clinical problem that often leads to significant morbidity in patients. Therapies that augment surgical repair may be beneficial to improve functional outcomes. Macrophage polarization is the process by which macrophages take on phenotypically distinct functions based on the local environment and signaling cues following peripheral nerve injury. Exercise has been shown to drive macrophage polarization from a pro-inflammatory $\mathrm{M} 1$ phenotype towards an anti-inflammatory M2 phenotype in numerous tissues, but remains uninvestigated in the peripheral nervous system.

Methods: The purpose of our study was to determine if exercise affects macrophage polarization, motor and sensory function, and neuroregeneration following sciatic nerve crush. Male and female C57BL/6 mice underwent sciatic nerve crush injury and were then given access to running wheels (exercised) or not given access to running wheels (sedentary) for 4 weeks. Analysis included behavioral assessments and anatomical studies.

Results: Exercised mice ran an average of $2.9 \mathrm{~km}$ per night. Injured exercised mice were protected from the development of thermal hyperalgesia and had fewer paw slips on beam walk testing. No differences were measured in mechanical sensitivity or motor coordination and balance. Motor nerve conduction velocities from injured exercised animals were significantly higher than injured sedentary animals suggesting improved nerve recovery with exercise. Injured sciatic nerves from exercised mice demonstrated increased M2 macrophages at the crush site compared to sciatic nerves from injured sedentary mice. RT-PCR demonstrated increased Arg1 and TREM2 in the injured sciatic nerve following exercise. The behavioral changes and altered macrophage polarization correlated with increased epidermal nerve fiber density and improved myelination from injured exercised animals. In vitro nerve outgrowth studies supported the results.

Conclusion: Exercise likely alters macrophage polarization towards an anti-inflammatory phenotype which improves repair and recovery of the injured peripheral nerve.

382 Distal Nerve Transfer for Median Thenar Palsy: A Cadaveric Study

Hussam Abou-Al-Shaar, MD; Geoffrey T Dorius, BS; David A. Morton, PhD; Mark Alexander Mahan, MD

Introduction: Severe median nerve palsies often result in irreversible thenar atrophy and thumb abduction loss. The only current standard treatment options are tendon transfers, which are associated with extensive hand therapy and moderate success rate. To date, there are no reported distal nerve transfer options for thenar palsy associated with median nerve injuries.

Methods: Sixteen embalmed cadaveric hand specimens were dissected to demonstrate the anatomy of the thenar branch of the median nerve and the deep ulnar branches. The deep ulnar branch to the third lumbrical muscle was assessed for transfer to the thenar branch of the median nerve. Coaptation success and nerve diameters were calculated and analyzed.

Results: In all sixteen hands, the ulnar branch of the third lumbrical muscle could be coaptated to the thenar branch of the median nerve. Diameter mismatch was reduced by sectioning and eliminating the sensory division of the thenar branch of the median nerve. The diameter of the ulnar branch to the third lumbrical muscle was $0.8 \pm 0.2 \mathrm{~mm}$ while the median branch to the thenar muscles was $1.2 \pm 0.3 \mathrm{~mm}$.

Conclusion: This is the first anatomic study that shows the potential feasibility of distal nerve transfer. This technique was performed in one patient with thenar palsy resulting from a high median injury with poor prognosis for thenar recovery. Clinical utility of this surgery is yet to be determined. This ulnar to median distal nerve transfer may potential add to the potential options for re-establishing thenar abduction after high median nerve injuries.

\section{Pathologic Remodeling in Human Neuromas}

Hussam Abou-Al-Shaar, MD; Michael Karsy; Cheryl Ann Palmer; Mark Alexander Mahan, MD

Introduction: Neuroma pathology is currently described as "disorganized" without evidence of any comprehensible internal structure. We have previously observed evidence that there are consistent architectural elements to neuromas.

Methods: Human neuroma samples obtained clinically from surgical repair. Surgical specimens were investigated histopathologically and immunohistochemically. Human nerve samples that were harvested for other reasons were utilized as controls.

Results: Twenty-six specimens were studied: seven were control, thirteen were neuroma-in-continuity, four were stump neuromas, and two were avulsions. There were no substantial histopathologic differences between stump neuromas and neuromas-in-continuity except that neuromas-in-continuity had an intact fascicle or more in the specimen. On immunohistochemical analysis of the neuromas, the extracellular protein laminin demonstrated a consistent double lumen configuration. The outer lumen also stained with GLUT1 antibodies, a marker of perineurial cells. Antibodies to the neurofilament NF200 revealed small clusters of axons within the inner lumen, along with a relatively regular pattern of Schwann cells, as identified by the anti-S100 antibody. There was no significant CD68 staining in any sample, whether the neuroma was acute or chronic. Specimens from nerve avulsion injuries, however, had none of the features of the neuromas and had an architecture similar to control nerves.

Conclusion: Together, these immunohistochemical stains suggest micro-fascicle formation within the neuroma. Both stump and in-continuity neuromas demonstrated conserved consistent pattern of remodeled microfascicles. The regenerative cascade after nerve injury may contribute to this histopathology.

384 Digital Phenotyping in Patients with Spine Disease: A Novel Approach to Quantifying Mobility and Quality of Life

David J Cote, BS; Ian Barnett, PhD; Yi Lu, MD, PhD; JukkaPekka Onnela; Timothy R. Smith, MD, PhD, MPH

Introduction: Current methods for evaluating life quality and symptom severity in spine patients are mostly limited to surveybased Patient Reported Outcome Measures (PROMs), which rely on episodic, subjective patient reports and are subject to recall bias. Digital phenotyping is a novel approach for the moment-bymoment quantification of the individual-level human phenotype in situ using data from personal digital devices, in particular smartphones. It allows for objective measurement of social and behavioral markers and makes it possible to investigate patientcentred outcomes, including quality of life, in naturalistic settings.

Methods: Patients with clinically diagnosed spine disease were enrolled, and a smartphone application (BieweTM) designed for digital phenotyping was installed. This application collected passive meta-data on a minute-to-minute basis, including GPS, WiFi, accelerometer, text and phone logs, and screen on/off time. It also administered Visual Analogue Scale (VAS) daily pain surveys and collected a weekly voice sample. A Linear Mixed Model (LMM) framework was used to test for associations between daily self-reported pain and mobility/sociability inferred from passively collected data. 
Results: 105 patients were enrolled with a median follow-up time of 94.5 days. The daily pain survey response rate was $43 \%$ across all patients. GPS data was available on $93 \%$ of days. After correcting for multiple testing, three daily GPS summary statistics (average flight length, maximum diameter travelled, total distance travelled), each capturing different aspects of daily patient mobility, were statistically significantly associated with patient-reported pain. On average, an increase in pain by 0.23 was associated with a 10-fold decrease in the average length of a patient's flights on that same day ( $\mathrm{p}=0.002)$; an increase in pain by 0.14 was associated with a 10-fold decrease in a patient's maximum diameter $(\mathrm{p}=0.004)$; and an increase in pain by 0.13 was associated with a 10 -fold decrease in a patient's distance travelled over the course of a day $(\mathrm{p}=0.004)$.

Conclusion: Patients with spine disease reporting increased pain demonstrate reduced mobility as measured by passively collected smartphone GPS data. Smartphone-based digital phenotyping appears to be a promising and scalable approach to assess mobility and quality of life in patients with spine disease.

385 Benign Nerve Sheath Tumors of the Spine: Epidemiology, Demographic Factors, and Rate of Surgical Intervention

Nader Delavari, MD; Shengyang Wu; Nikhil Sahasrabudhe; Anthony Frempong-Boadu, MD

Introduction: Benign nerve sheath tumors arise from Schwann cells, fibroblasts, and perineural cells. They represent a rare group of tumors arising from the spinal nerve roots. The purpose of this study is to describe the epidemiology, demographic factors, and rate of surgical intervention of benign nerve sheath tumors of the spine.

Methods: Retrospective analysis of the Surveillance, Epidemiology, and End Results registry, a large populationbased tumor database, during a 10 year time period (2004-2014). The Kaplan-Meier model was utilized to determine overall and disease-specific survival.

Results: A total of 2007 patients were identified. Median age at diagnosis was 52 years. There was no significant difference in sex (female $47 \%$ ). Average tumor size was $3.18 \mathrm{~cm}$. Median follow up time was 59 month. $85 \%$ of patient's underwent surgery (1694/2007). Surgery was performed in $84.4 \%$ of cases. When compared to observation alone, surgery was associated with a higher probability of 10 year survival ( $73 \%$ vs $67.5 \%, p=0.0003)$. There was no significant difference in survival between gross total and partial resection. Only 20 patients underwent radiation therapy $(1 \%)$.

Conclusion: Surgical intervention was associated with a higher probability of 10 year survival. However, there was no significant difference in survival between gross total and partial resection.

386 Fusion for Spinal Instability after Peripheral Nerve Tumors Surgical Resection with Facetectomy

Mohammed Adeeb Sebai; Jang W Yoon, MD., MS., BS.; Panagiotis Kerezoudis; Mohammed Ali Alvi, MD; Sandy Goncalves; Robert J. Spinner, MD; Mohamed Elminawy, MBBCh; Mohamad Bydon, MD

Introduction: Peripheral Nerve Sheath Tumors (PNSTs), including shawannomas and neurofibromas are a group of uncommon tumors originating from nerve and related structures. Standard surgical management for PNST includes facetectomy to allow adequate resection. Herein, we have presented the largest single- institutional experience as well as a literature review to assess the factors associated with having a spinal fusion, compared to facetectomy alone.

Methods: Institutional medical records and a prospectively maintained cancer registry were queried for all cases of PNSTs. For our study, we included subjects which were managed surgically and categorized into those undergoing facetectomy alone and those undergoing facetectomy plus immediate fusion. Variables of interest included age at the time of surgery, surgery date, tumor size, tissue diagnosis, total operated levels and reason for fusion as per the surgeon's note, and clinical outcomes.

Results: Fifty-six patients fit our inclusion criteria. Demographics were evenly distributed among the 2 groups. The most common tumor was found to be schwannoma in both groups $(75 \%$ in non-fusion group vs $85.7 \%$ in the fusion group, $\mathrm{p}=0.50$ ). Tumor size was found to be similar in both groups $(27.56 \mathrm{~mm} \pm 13.44$ in non-fusion vs $31.96 \mathrm{~mm} \pm 14.68$ in fusion group, $\mathrm{p}=0.2590$ ). Compared to those undergoing facetectomy alone, patients undergoing fusion were found to have higher odds of having total facetectomy than subtotal facetectomy $(\mathrm{OR}=6.15$, $95 \% \mathrm{CI}=1.19-31.82)$ and having thoracic involvement( $\mathrm{OR}=2.25$, $95 \% \mathrm{CI}=0.72-7.01)$. The most common indication for fusion as per surgeon's assessment was found to be the amount of bone removal. Reoperation rate was similar for both groups. Only one patient who received fusion $(3.5 \%)$ had pseudarthroses.

Conclusion: Our results indicate that patients with PNST who have thoracic involvement and require total facetectomy are more likely to get fusion. Moreover, the amount of bone removal leading to instability was found to be the most common indication for fusion compared to facetectomy alone.

387 Chronic in Vivo Imaging of Intact Corticospinal Motor Neurons Shows Functional Reorganization During Recovery from Contralateral Spinal Cord Injury

David Adam Gimbel, MD, MHS; Cafferty William, PhD; Stephen Strittmatter, MD/PhD

Introduction: Spinal cord injury (SCI) results in chronic functional deficits as axons in the CNS fail to regenerate after injury. Despite the lack of long distance axon regeneration after injury, humans with partial spinal cord injury can show spontaneous recovery of function. Structural plasticity of intact/uninjured axon tracts has been implicated as the driver of this phenomenon. Previous data from our laboratory has shown that lack of Nogo Receptor signalling allows sprouting from the intact CST to innervate the denervated cord. This sprouting results in functional improvement. Using a combination of limb kinematics and chronic in vivo two-photon calcium imaging of intact corticospinal motor neurons, we investigate the spatial and temporal firing rates of forelimb movement during a CST dependent task before and after contralateral injury.

Methods: GCaMP6 is a family of ultrasensitive protein calcium sensors that fluoresce with calcium influx into neurons. Animals with GCaMP6 specific for layer 5 pyramidal neurons had cranial windows placed over motor cortex. Cellular firing patterns were mapped in intact awake animals using a combination of limb kinematics and in vivo two-photon imaging. The kinematics were then mapped after recovery from injury.

Results: Chronic in vivo two photon imaging is a viable way to map action of motor neurons and their function before and after CNS injury. By using limb kinematics individual cell firing patterns for limb movement can be established and changes can be identified after recovery from CNS injury.

Conclusion: Sprouting from intact spinal neuronal pathways is responsible for recovery of function after spinal cord injury. By 
mapping firing CST neurons in the cortex and correlating the firing with function the nidus of recovery can be explained.

388 Neuromodulation in Chronic Cervical Spinal Cord Injury: Electromyogram Features of Stimulation-assisted Upper-limb Volitional Function

Nicholas $\mathrm{Au}$ Yong, MD, PhD; Yevgeniy Freyvert, MD; Reggie Edgerton, $\mathrm{PhD}$; Morteza Modaber, MD; Erika Morikawa; Sharon Zudnowski; Melanie Sarino; Majid Sarrafzadeh, PhD; Marc Nuwer; Roland R Roy; Yury Gerasimenko; Daniel C. Lu, $\mathrm{MD}, \mathrm{PhD}$

Introduction: Neuromodulation through electrical stimulation is clinically employed in the central nervous system for treatment of movement disorders and pain. More recently, epidural stimulation (ES) has been used for partial restoration of lower-limb motor function in spinal cord injured human subjects. In this study, we demonstrate that ES can also promote volitional hand function in human subjects with chronic cervical cord injury. Electromyogram features of hand function recovery were characterized to elucidate the underlying mechanism.

Methods: Two chronic ( $>18$ mo from injury) American Spinal Injury Association (ASIA) Impairment Scale (AIS) B subjects, each implanted with a cervical epidural array of 16 electrodes for chronic pain, were evaluated for improvement of handgrip control with ES. An electronic handgrip force measurement device was used to measure voluntary hand control and grip characteristics. Surface electromyogram activity was measured concurrently. Various clinical metrics were also assessed throughout the study.

Results: Hand control and force production improved in both subjects as compared to baseline function. Both metrics were sustained even after cessation of stimulation. Improved hand function was associated with increases in electromyogram amplitudes. ES produced evoked responses predominantly during voluntary effort. The amount of force produced corresponded best with combined stimulus evoked (i.e. direct stimulation) and non-evoked electromyogram activity, as opposed to stimulus evoked alone.

Conclusion: Cervical electrical modulation merits further investigation as a means to improve hand volitional function in patients with chronic tetraplegia. These results suggest that the cervical motor circuitry, while operationally dissimilar to the locomotor central pattern generator, is also amenable to neuromodulation through electrical stimulation. Features of electromyogram activity point to a mechanism whereby electrical stimulation augments the excitability of premotor circuits by shifting the network's state closer to motor threshold.

389 Lack of Rhomboid Muscle Volume Is Associated with Significantly Decreasesd Arm Function in Long-term Followup of Neonatal Brachial Plexus Palsy

Lynnette Rasmussen, OTR/L; Brandon W Smith, MD, MSCR; Kate Wan-Chu Chang, MA; Denise Justice, OTR/L; Thomas J. Wilson, MD; Lynda Jun-San Yang, MD, PhD

Introduction: The rhomboids stabilize the scapula, which serves as the origin for key muscles targeted in neonatal brachial plexus palsy (NBPP) repair. Since the association of rhomboids with functional arm recovery remains unexplored, we evaluate the association of early rhomboid muscle volume (RHV) with longterm arm function.

Methods: We compared the active range of motion (AROM) at the shoulder and elbow in patients with normal vs decreased RHV in a retrospective cohort study. A single radiologist used ultrasound to determine $\mathrm{RH}$ volume at $2-4 \mathrm{yrs}$ of age. AROM of shoulder flexion, abduction, external rotation, and elbow flexion was measured at 2-4 years and at 6-10yrs. We applied Student's T-test to compare the AROM differences between the 2 groups.

Results: 36 patients with NBPP were identified: 8 with decreased and 28 with normal RHV. Shoulder external rotation was significantly increased in patients with normal vs decreased RHV ( $33^{\circ}$ vs $-29^{\circ}, \mathrm{P}=0.001$ ); likewise, shoulder flexion, abduction, and elbow flexion in abduction were also significantly increased with normal RHV $\left(35^{\circ}\right.$ vs $93^{\circ}, \mathrm{P}=0.021 ; 131^{\circ}$ vs $85^{\circ}$, $\mathrm{P}=0.016 ; 142^{\circ}$ vs $\left.108^{\circ}, \mathrm{P}=0.014\right)$. At $6-10$ years of age, external rotation remained significantly increased in the normal RHV group ( $38^{\circ}$ vs $\left.-20^{\circ}, \mathrm{P}=0.007\right)$ : again, shoulder abduction, external rotation, and elbow flexion in adduction were significantly better in the normal RHV group $\left(144^{\circ}\right.$ vs $99^{\circ}, \mathrm{P}=0.044 ; 136^{\circ}$ vs $105^{\circ}$, $\mathrm{P}=0.015)$. Continued improvement in AROM occurred from $2-4 y r s$ to 6-10yrs in both groups; no significant differences in mean change of improvement was seen.

Conclusion: We provide the first data that RHV directly influences arm function recovery in NBPP.

390 Chronic Nerve Interfacing Utilizing Graft-embedded Regenerative Electrodes

Amrita Nishtala, MS; Ying Yan, MD, PhD; Nikhil Chandra, MS; Nathan Birenbaum, BS; Matthew R MacEwan, BSE, PhD; Wilson Zachary Ray, MD

Introduction: Regenerative sieve electrodes represent a novel means of facilitating chronic high-specificity nerve stimulation needed to restore sensorimotor function following neurological injury. Yet, implementation of regenerative sieve electrodes commonly requires transection of target nerves during microeletrode implantation. The present study aimed to examine the efficacy of a new surgical technique in which regenerative sieve electrodes were integrated into peripheral nerve grafts and applied in an endto-side neurorrhaphy to target nerves as a means of facilitating a stable, high-selectivity interface to peripheral motor axons.

Methods: Custom-designed macrosieve electrodes were fabricated out of polyimide and gold using sacrificial photolithography. Regenerative sieve electrodes were then microsurgically implanted into $20 \mathrm{~mm}$ nerve autografts harvested from the sciatic nerve of male Lewis rats. Electrode-enabled nerve grafts were microsurgically implanted in an end-to-side manner into donor rat sciatic nerves without the need for transection the host nerve. Nerve interfacing was assessed in situ by selectively stimulating regenerated nerve tissue via implanted sieve electrodes while simultaneously mapping activation evoked muscle activation and force production at 3 months post-operatively.

Results: Micro-surgical implantation of electrode-enables nerve grafts into the sciatic nerve of healthy male rats for 3 months resulted in robust axonal regeneration through the macrosieve electrode. Electrophysiological assessment confirmed the preservation of motor function over 3 months post-operatively. Chronically implanted macrosieve electrodes demonstrated successful and independent recruitment of motor nerve fibers and induction of selective muscle activation in independent muscle groups in the lower limb.

Conclusion: The present study highlights the first instance of regenerative electrodes achieve a chronic interface to a target nerve without the need for transecting the target nerve. These findings represent a significant advance in the surgical application of neuroprosthetic technologies and support the continued development of advanced biohybrid neural interfaces. 


\section{High Frequency Alternating Current (HFAC) Nerve Block Utilzing Regenerative Macrosieve Electrodes}

Soumyajit Ray, MS; Nathan Birenbaum, BS; Ying Yan, MD, PhD; Nikhil Chandra, MS; Matthew R MacEwan, BSE, PhD; Wilson Zachary Ray, MD

Introduction: High frequency alternating current (HFAC) block has been shown to be effective for blocking peripheral nerve conduction, and could potentially be used to block undesirable neural activity or to ensure unidirectional transduction of exogenously elicited action potentials. Prior studies have demonstrated HFAC block using cuff electrodes, intrafascicular electrodes, and glass suction electrodes. Regenerative macrosieve electrodes represent a novel means of facilitating chronic high-specificity nerve stimulation needed to restore sensorimotor function following neurological injury, yet have not been investigated as a platform for inducing HFAC in peripheral nerves. In this study, optimal methods of inducing HFAC utilizing novel regenerative macrosieve electrode assemblies (DMSEAs) was investigated in a rodent sciatic nerve model.

Methods: Custom-designed DMSEAs were fabricated using sacrificial photolithography. Regenerative DMSEAs were then microsurgically implanted into the sciatic nerve of male Lewis rats. The efficacy of various DMSEA stimulus configurations was tested in acute experiments for HFAC block of stimuli from a proximally located bipolar cuff electrode. Force measurements from the tibialis anterior muscle were used to determine the presence or absence of HFAC block.

Results: Sinusoidal HFAC block signals were tested with frequencies ranging from $10 \mathrm{kHz}$ to $42 \mathrm{kHz}$, and with voltages ranging from $2 \mathrm{~V}$ to $20 \mathrm{~V}$ p-p. A complete and quickly reversible block was obtained using the DMSEAs in the joint assembly as an anode/cathode pair (anode proximal, cathode distal). The frequency voltage characteristic of the block was mapped. The block threshold, defined as the minimum voltage required at a particular frequency to ensure a complete block, was observed to increase as the frequency increased.

Conclusion: The present study highlights the first instance of regenerative electrodes successfully inducing a HFAC nerve block. These findings demonstrate the potential of DMSEAs to perform critical neuromodulatory functions and provide a stable, high-selectivity interface useful in advanced neuroprosthetic systems.

392 Nerve Deficits after Stab Wounds to the Upper Extremity Due to Compartment Syndrome: A Case Series

\section{Gabriel Claudiu Tender, MD}

Introduction: Stab wounds resulting in neural deficit with a specific distribution are considered surgical emergencies, since sharp nerve transection can be repaired primarily, with improved results compared to graft repair. However, we report four such cases in which the nerve deficit was due to compartment syndrome rather than direct nerve laceration.

Methods: Four patients with knife stab wounds to the arm $(n=1)$ and forearm $(n=3)$ presented to the emergency room within hours from the injury. The physical examination revealed a complete radial nerve deficit in the patient stabbed in the arm and complete median, ulnar, and anterior interosseus nerve deficit, respectively, in the patients stabbed in the forearm. All patients were taken to the operating room emergently for exploration and possible repair.

Results: The culprit nerve was identified and exposed proximal and distal to the level of injury in all 4 patients, after evacuat- ing large hematomas under pressure. Intraoperative nerve action potential recording showed good conduction across the level of injury, negating the need for nerve repair. All patients showed rapid postoperative improvement in nerve function.

Conclusion: Occasionally, stab wounds may result in indirect nerve injury, via compartment syndrome. These cases remain surgical emergencies, since immediate nerve decompression results in functional recovery. Anatomic identification proximal and / or distal to the level of injury is crucial, since the nerve can rarely be found in the distorted field of injury.

393 The Barrow Biomimetic Spine: Fluoroscopic Analysis of a Synthetic Spine Model Made of Variable 3D-printed Materials and Print Parameters

Michael Bohl, MD; Michael Anthony Mooney, MD; Peter Nakaji, MD; Steve W. Chang, MD; Jay D. Turner, MD, PhD; Udaya K. Kakarla, MD

Introduction: The Barrow Biomimetic Spine project aims to develop a 3-dimensional (3D) printed, synthetic spine model that will one day replace cadaveric tissue in spine biomechanical research. A crucial component to any biomimetic spine model is that it performs similarly to cadaveric tissue on standard diagnostic imaging modalities. The purpose of this study is to analyze the fluoroscopic performance and fidelity to human tissue of a new synthetic spine model.

Methods: Numerous L5 vertebral bodies were 3D printed with variable shell thicknesses and internal densities, and fluoroscopic images were taken of these models to measure cortical thickness and gray-scale density. An L3-L5 spinal segment was then printed, and fluoroscopic films were obtained at variable $\mathrm{C}$-arm angles. Three spine surgeons subjectively scored these images for human fidelity. Pedicle screws were then placed into the L3-L5 segment to demonstrate successful or breached placement. Standard anteroposterior (AP) and lateral films were taken, and 3 spine surgeons were tested and scored on correctly identifying screw placement.

Results: Cortical thickness and gray-scale density testing demonstrated an upward trend with increases in relevant print settings. Subjective scoring demonstrated nearly perfect fidelity for the L3-L5 model. Surgeon identification of screw placement on the AP and lateral fluoroscopic views also demonstrated nearly perfect fidelity.

Conclusion: This study is the first to demonstrate that 3D-printed vertebral body and segmental spine models accurately mimic human tissue on C-arm fluoroscopy, both in respect to their anatomical appearance in standard views, but also in their response to surgical manipulation and the variations in $\mathrm{C}$-arm angle that commonly occur in the operating room. As such, these spine models have the potential to serve as an excellent platform for future research and surgical education programs.

394 Incidence of Durotomy in Lumbar Spinal Surgery: A Barrow Neurological Institute Prospective Data Analysis

Benjamin Hendricks, MD; Aabra Ahmed, BS; Laura A. Snyder, MD

Introduction: Incidental durotomy is a complication observed in up to $17 \%$ of spinal surgeries, specifically ranging from 2 to $20 \%$ in lumbar degenerative spinal surgery. Correlational factors for incidental durotomy have been controversially published which include age greater than 65 years, obesity, female gender, degenerative spondylolisthesis, revision surgery, and surgeon experience. This prospective analysis was undertaken with the objective of demonstrating the impact of body mass index (BMI) 
on the incidence of durotomy during degenerative lumbar spinal procedures, and exploration of additional correlational factors.

Methods: Analysis was performed on a prospective observational registry of 506 patients undergoing lumbar spinal surgery for primary or recurrent lumbar degenerative disease from the National Neurosurgery Quality and Outcomes Database (N2QOD) from our institution. Statistical analysis was performed using GraphPad Prism 7.03, for calculation of descriptive statistics and Pearson correlation coefficients.

Results: The N2QOD lumbar spinal procedure database is composed of 506 patients prospectively analyzed with a mean age of $60.2(+/-13.8)$ years, mean body mass index $30.0(+/-$ 5.52 ), lumbar spinal fusion procedure rate of $43.7 \%$ (221 cases), incidental durotomy $13.0 \%$ (66 cases), and female gender $51.2 \%$ (259 patients). BMI demonstrates a Pearson correlation value $r$ $=0.097(\mathrm{p}=0.029)$, suggestive of a small positive correlation with durotomy. Age $(r=0.073, \mathrm{p}=0.102)$ and female gender $(\mathrm{r}=0.014, \mathrm{p}=0.748)$ were not significantly correlated with the incidence of durotomy. Lumbar fusion procedures $(\mathrm{r}=-0.116, \mathrm{p}=$ $0.009)$ showed a weak negative correlation.

Conclusion: This study demonstrated a weakly positive correlation between durotomy and BMI. Literature on this topic demonstrates mixed results and within this series it is unclear if BMI significantly impacts durotomy rates. Similarly, other published correlative factors impacting the rate of durotomy were not demonstrated to be a significant contributor in this study.

395 Incidence of C5 Palsy after Posterior Cervical Procedures: Analysis of Risk Factors Using Pre-operative Clinical and Radiographic Measurements

Yi-Ren Chen, MD, MPH; Eli Johnson, BS; Katie Shpanskaya; John K. Ratliff, MD, FACS; Atman Desai, MD, MA; Maziyar Arya Kalani, MD; Jon Park; Harminder Singh, MD

Introduction: C5 nerve root palsy after cervical spinal surgery is a serious complication leading to weakness and paralysis of the deltoid and bicep. However, preoperative risk factors are not fully understood.

Methods: A retrospective review was conducted over 2 years at a single institution involving patients receiving posterior cervical decompression. We included all patients undergoing posterior cervical laminectomy with laminoplasty or posterior fusion from $9 / 2014$ to $9 / 2016$. We identified 238 patients and 42 were removed by the exclusion criteria. Exclusion criteria included surgery not involving the C4-C5 level, anterior surgery at the C4-C5 level within the last 6 months and less than one year of follow-up.

Results: The incidence of C5 palsy was $5.10 \%(\mathrm{~N}=10)$. The C5 palsy group had significantly greater age (72.20 vs. 63.96; $p=0.047)$, male predominance $(100.00 \%$ males vs $67.74 \%$ males; $\mathrm{p}=0.033$ ), greater comorbidity with coronary artery disease $(50.00 \%$ vs $20.43 \% ; \mathrm{p}=0.028)$, greater presence of preoperative myelomalacia $(40.00 \%$ vs $12.37 \%$; $\mathrm{p}=0.014)$, and lower body mass index (26.52 vs 28.27; $\mathrm{p}=0.002)$. C5 palsy patients also had narrower preoperative sagittal $(p=0.026)$ and axial $(p=0.049)$ spinal canal widths. Spinal cord congestion, measured as preoperative sagittal $(p=0.035)$ and axial $(p=0.048)$ spinal cord width as a percentage of total spinal canal width, was greater in the $\mathrm{C5}$ palsy group. Instrumentation did not appear to be correlated with C5 palsy $(75.8 \%$ rate in the no palsy group, vs $70 \%$ in the palsy group; $\mathrm{p}=0.65$ ).

Conclusion: The incidence of C5 nerve root palsy was $5.10 \%$ in our cohort of 196 patients. Increased age, male gender, myelomalacia, coronary artery disease, narrower preoperative spinal canal width and spinal cord congestion on MRI were associated with increased incidence of C5 palsy. This might help guide pre- operative counselling and risk assessment of patients undergoing posterior cervical decompression and/or fusion procedures based on demographics, associated co-morbidities and pre-operative MR imaging.

\section{The Lost Art of Posterior Cervical Microdiscectomy}

Jared Ament, MD, MPH; Tejas Karnati; Edwin Kulubya, MD; Harjot Thind, MD, MPH; Kee Duk Kim, MD; J. Patrick Johnson, MD, MS

Introduction: Although in recent decades the surgical treatment of cervical radiculopathy has centered around the anterior cervical discectomy and fusion operative technique, posterior cervical microdiscectomy has been underutilized despite the costeffectiveness of the procedure and similar clinical outcomes when compared to anterior approaches.

Methods: A thorough review of existing studies was conducted. Studies that compared direct and indirect costs of ACDF and PCM, reoperation rates, and outcome measurements were found from a simple search on Pubmed, Medline and Embase databases.

Results: Direct costs of ACDF often are significantly higher than PCM. Furthermore, a 1-year cost-utility analysis demonstrated that ACDF had \$131,951/QALY gained while PCM had $\$ 79,856 /$ QALY gained. Reoperation rates of $\mathrm{PCF} / \mathrm{PCM}$ range from $2 \%$ to $9.8 \%$ while ACDF ranges from $2 \%$ to $8 \%$. Furthermore, there is no significant difference between the reoperative rates of PCM versus ACDF.

Conclusion: Posterior cervical microdiscectomy is not only a safe, effective operative technique for the surgical treatment of cervical radiculopathy, but it is also a more cost-effective procedure with similar clinical outcomes when compared to the anterior cervical discectomy procedure.

397 Imaging Characteristics Predict Operative Difficulty Mobilizing the Sciatic Nerve for Proximal Hamstring Repair

Thomas J. Wilson, MD; B. Matthew Howe; Robert J. Spinner, MD; Aaron Krych, MD

Introduction: Repair of proximal hamstring avulsions requires mobilization of the sciatic nerve away from the tendon stump, which can be achieved with varying difficulty depending on the degree of scar formation and adherence. Predicting when a scarred, adherent, difficult-to-mobilize nerve will be encountered has been difficult. The purpose of this study was to identify clinical and/or radiologic factors predictive of a difficult intraoperative dissection of the sciatic nerve during proximal hamstring repair.

Methods: We retrospectively reviewed the medical records and preoperative MRIs of consecutive patients undergoing proximal hamstring repair. We compared the groups with and without a difficult sciatic nerve dissection.

Results: The total cohort consisted of 67 patients. Factors found to increase the likelihood of a difficult sciatic nerve dissection included complete conjoint tendon avulsion, higher maximal amount of tendon retraction, higher degree of imaging abnormality in the sciatic nerve, and higher degree of circumferential relationship of hematoma to the sciatic nerve. At a threshold of 23 , for the Sciatic Nerve Dissection Score, the positive and negative predictive values were $53 \%$ and $88 \%$, respectively. For the decision tree, the positive and negative predictive values were $75 \%$ and $87 \%$, respectively.

Conclusion: We have identified imaging factors associated with a scarred, adherent sciatic nerve that predict a difficult dissection during proximal hamstring repair. We have developed two novel methods the Sciatic Nerve Dissection Score and a decision 
tree that can be applied to predict the probability of a difficult sciatic nerve dissection at the time of surgical repair.

398 Evaluation of Patient-initiated Spontaneous Arm Use in Adults After Nerve Transfer for Brachial Plexus Palsy

Brandon W Smith, MD, MSCR; Kate Wan-Chu Chang, MA; Serena Saake; Lynda Jun-San Yang, MD, PhD; Susan Brown, Ph.D.

Introduction: Nerve transfers (NT) for brachial plexus palsy (BPP) demonstrate encouraging outcomes via outpatient clinic evaluation, but quantification of patient-initiated spontaneous arm use remains elusive. Whether NT should focus more upon shoulder vs elbow recovery is controversial. We employed novel motion sensor technology to capture spontaneous arm movement after NT.

Methods: This pilot study comprised BPP patients who underwent NT and reported self-perceived arm use via DN4, SPADI, DASH, Abilhand, IPAQ, and PROMIS. Standard active range of motion (AROM) and passive range of motion (PROM) was performed. To detect spontaneous arm use at home / work, Actigraph GT9X monitors were worn bilaterally for 7 days which captured frequency (VT), and quality (VM) of movement. Data were expressed as a ratio of the affected arm to the unaffected arm.

Results: Five males (mean age $=41 \mathrm{yrs}+/-17 \mathrm{yrs}$ ) with average time from surgery of $2 \mathrm{yrs}(+/-1 \mathrm{yr})$ participated. Overall, mean VT was $0.73(+/-0.27)$, and mean VM was $0.63(+/-0.59)$. Shoulder flexion and abduction AROM correlated significantly with VT (0.97, $\mathrm{P}=0.008 ; 0.99, \mathrm{P}=0.002)$, whereas no significant correlation existed with elbow flexion $(0.73, \mathrm{P}=0.17)$ or forearm supination (0.43, $\mathrm{P}=0.47)$. Similarly, both shoulder flexion and abduction AROM correlated significantly with VM $(0.93, \mathrm{P}=0.022 ; 0.96$, $\mathrm{P}=0.009)$, whereas no correlation elbow flexion $0.66(\mathrm{P}=0.23)$ or forearm supination $0.39(\mathrm{P}=0.52)$ with $\mathrm{VM}$ was seen. VT and VM also correlated strongly with neuropathic pain (DN4).

Conclusion: Novel use of motion sensor technology allows quantitative assessment of patient-initiated spontaneous arm use: high VT and VM supports continued use of NT in BPP. We provide the first data to demonstrate that both frequency and quality of arm movement rely more on shoulder than elbow AROM.

399 Identifying Diffusion Tensor Imaging Biomarkers in Cervical Spondylotic Myelopathy

Michael John P Mendoza, BS; Mayank Kaushal, MD, MBA; Avinash Rao, BS; Matthew Budde, PhD; Shekar N. Kurpad, MD, $\mathrm{PhD}$; Marjorie C. Wang, MD, MPH

Introduction: We examined the potential of Diffusion Tensor Imaging (DTI) to predict functional outcomes in patients undergoing surgery for Cervical Spondylotic Myelopathy (CSM). There are no valid imaging biomarkers for predicting the efficacy of surgery in CSM. We examined preoperative DTI metrics in a large cohort of CSM patients. We correlated these metrics to Preoperative mJOA scores as well as postoperative clinical responses to evaluate their utility as biomarkers in CSM.

Methods: 72 patients with CSM were enrolled and underwent MRI prior to surgery. Functional outcomes were measured using the mJOA at pre-op, 3, 6, 12, and 24 months post-operation. DTI metrics of fractional anisotropy (FA), mean diffusivity (MD), and transverse (tADC) and longitudinal apparent diffusion coefficient (lADC) were measured at the level of maximal compression and in the whole-cord. Measures of spinal cord cross-sectional dimensions were also taken. MRI parameters were related to mJOA scores, with an improvement greater than 2 being considered clinically significant.
Results: On average, mJOA score improved after 3 months post-op, without further change afterwards. Initial mJOA was found to be negatively correlated with change in mJOA score at each time point. None of the DTI markers had significant correlations with either initial mJOA or change in mJOA (?mJOA) at any timepoint. Subjects were further classified as having either a clinically significant improvement independent of mJOA score at 12 months (responders) or not (non-responders). Responders had a significantly lower $1 \mathrm{ADC}(\mathrm{p}=0.017)$ and $\mathrm{MD}(\mathrm{p}=0.039)$ than non-responders.

Conclusion: While not correlating with mJOA scores, DTI has shown the ability to discriminate patients into those that show a significant improvement from surgery from those that do not. Additional studies with larger sample sizes are needed to define the accuracy and clinical relevance of DTI in predicting recovery in CSM.

400 Home vs Rehab: Factors That Influence Disposition After MIS Adult Spinal Deformity Surgery?

Justin Ledesma, MD; Robert Eastlack, MD; Stacie Tran, BS, MPH; Paul Park, MD; Khoi Duc Than, MD; Praveen V. Mummaneni, MD; Dean Chou, MD; Adam S. Kanter, MD; Neel Anand, MD; Pierce D. Nunley MD; Frank La Marca MD; Richard G. Fessler MD, , PhD; Juan S. Uribe MD,

Introduction: MIS correction for ASD may reduce the need the need for postoperative skilled nursing (SNF) or rehabilitation facility placement following surgery. The likelihood of requiring placement in a facility, rather than home disposition, may be influenced by a variety of factors. Additionally, the association of discharge location on outcomes and complication rates has not been elucidated in these patients. In this study, we aimed to define factors predicting disposition to $\mathrm{SNF} / \mathrm{rehab}$, and the rates of complications occurring in patients sent home vs. a facility.

Methods: Inclusion criteria: $>18$ yrs old, MIS as part of index surgery, location of discharge, and at least one of the following: PT $>20$, SVA $>5 \mathrm{~cm}$, PI-LL $>10$, or lumbar scoliosis $>20$. Patients with $2 \mathrm{yr}$ follow-up were included. Preoperative demographic and radiographic data, postop (<30 day) complications and HRQL were analyzed.

Results: 182 patients met inclusion criteria. 113 were discharged home (HOM) vs 69 to $\mathrm{SNF} / \mathrm{rehab}(\mathrm{REH})$. Older patients $(>50)$ were more likely to go to REH $(p=0.043)$. Those $>70$ yrs were 6 times more likely to go to REH. No association was identified among radiographic parameters except preop pelvic tilt (OR $=1.11$ to $\mathrm{SNF} / \mathrm{Rehab}, \mathrm{p}=0.009)$. Staged cases were more likely to $\mathrm{d} / \mathrm{c}$ to REH (OR 3.24; CI:1.11, 9.46; $\mathrm{p}=0.032$ ) otherwise there was no difference for levels treated, OR time, EBL, osteotomy or length of stay. Patients requiring REH had more complications $(58 \%$ v 39.8\%, $\mathrm{p}=0.017)$ including Major (19.5 v 42\%; $\mathrm{p}=0.001)$, perioperative $(14.2 \times 31.9 \% ; \mathrm{p}=0,004)$ and infections $(3.5 \mathrm{v} 13 \%$; $\mathrm{p}=0.016)$. REH patients had higher revision rates $(19.5 \%$ v $33 \%$; $\mathrm{p}=0.035$ ). (Table 1) HRQOL measures were similar regardless of disposition.

Conclusion: Older patients and those undergoing staged MIS deformity correction, have a higher likelihood of postoperative disposition to rehab/SNF. Complications occurred more commonly in those patients requiring SNF/rehab after hospitalization.

401 A Prognostic Nomogram for Outcomes Following Correction of Adult Spinal Deformity

Akshay Sharma, BA; Joseph E Tanenbaum, BA; Olivia Hogue; Syed K Mehdi, BS; Sagar Vallabh; Emily Hu, BA; Edward C. Benzel, MD; Michael P. Steinmetz, MD; Jason Savage, MD 
Introduction: Deformity reconstruction surgery has been shown to improve quality of life in cases of adult spinal deformity, but is associated with significant morbidity. We sought to create a preoperative predictive nomogram to help risk-stratify patients with adult spinal deformity and determine which would likely benefit from surgery

Methods: All patients aged 25-years and older with radiographic evidence of adult spinal deformity that underwent thoracolumbar fusion between 2008 and 2014 were identified. Demographic and clinical parameters were obtained. The EuroQol five dimensions questionnaire (EQ-5D) was used to measure health-related quality of life (HRQoL) preoperatively and at 12 months postoperative follow-up. A preoperative to postoperative improvement of .10 or greater was used to indicate the presence of clinically relevant improvement in HRQoL. Logistic regression of preoperative variables was used to create the prognostic nomogram.

Results: Our sample included data from 191 patients. 51\% of patients experienced clinically relevant postoperative improvement on the EQ-5D. Seven variables were included in the final model: preoperative EQ-5D score, sex, preoperative diagnosis (degenerative, idiopathic, or iatrogenic), previous spinal surgical history, obesity, and a sex-by-obesity interaction term. Preoperative EQ-5D score independently predicted the outcome. Sex interacted with obesity: obese men were at disproportionately higher odds of improving than non-obese men, but obesity did not affect odds of the outcome among women. Female sex was also shown to be a predictor of postoperative improvement but only among non-obese patients. Model discrimination was good, with an optimism-adjusted c-statistic of 0.739 .

Conclusion: Lower preoperative EQ-5D scores were associated with a clinically significant increase in postoperative EQ-5D scores and sex was found to interact with obesity when predicting post-operative EQ-5D scores. The predictive nomogram that we developed using these data can improve preoperative risk counseling and patient selection for deformity correction surgery.

\section{The Safety and Efficacy of CT-Guided, Fluoroscopy- free Vertebroplasty in Adult Spinal Deformity Surgery}

Corinna Clio Zygourakis, MD; Anthony Michael DiGiorgio, DO, MHA; Clifford Crutcher, MD; Michael Safaee, MD; Fred Nicholls; Cecilia L. Dalle Ore, B.A.; Vedat Deviren, MD; Christopher P. Ames, MD

Introduction: Proximal junctional kyphosis (PJK) is one of the most common, morbid, and costly complications of adult spinal deformity surgery. Our goal is to analyze the safety and efficacy of a novel technique of CT-guided, fluoroscopy-free vertebroplasty to help prevent PJK in long-segment spinal fusions.

Methods: Retrospective analysis of 118 consecutive adult spinal deformity patients who underwent long-segment fusion with vertebroplasty augmentation, 2013-2016, at a single institution. For each patient, we collected demographics, surgical information (anterior/posterior versus posterior approach, interbody fusion device, ligamentoplasty augmentation, decompression vs not), length of stay, discharge disposition, and complications, including reoperation, PJK, and PJK requiring reoperation. We reviewed all post-operative radiographs to assess for cement leakage from vertebroplasty. These patients were compared to a historical control of 253 patients who underwent adult spinal deformity surgery without vertebroplasty augmentation from 2004-2013 at our institution.

Results: 118 patients $(77 \mathrm{~F}, 41 \mathrm{M})$ underwent posterior spinal instrumentation with fluoroscopy-free vertebroplasty, the major- ity with ligamentoplasty, interbody fusion, and decompressions. More than half of the patients $(52 \%)$ had no radiographic evidence of cement leakage, and none of the patients were symptomatic from this leakage. The PJK rate of $14 \%$ and the PJK requiring re-operation rate of $3 \%$ in this cohort of patients who underwent vertebroplasty-augmented fusion was significantly lower than that of the 253 historical controls at our institution who did not undergo vertebroplasty (40\% PJK rate, $17 \%$ PJK-rate requiring re-operation; both $\mathrm{p}<0.001)$. After controlling for patient and other surgical factors in multivariate analyses, vertebroplasty was significantly associated with lower rates of PJK and PJK requiring re-operation $(\mathrm{p}<0.001$ and $\mathrm{p}=0.003)$.

Conclusion: Our novel vertebroplasty technique is safe and eliminates the need for additional fluoroscopy in cases already utilizing the $\mathrm{O}$-arm to verify screw placement. In addition, it is an effective technique for reducing PJK in adult spinal deformity surgery when compared to historical institutional controls.

403 Combined Lateral Transpsoas Anterior Column Realignment with Pedicle Subtraction Osteotomy: Cadaveric Feasibility and Early Clinical Experience

Jakub Godzik, MD, MSc; Randall Hlubek, MD; David S. Xu, MD; Corey Tyler Walker, MD; Jay D. Turner, MD, PhD; Gregory M Mundis; Juan S. Uribe, MD, FAANS

Introduction: Traditional approaches to the lumbar spine for deformity correction include pedicle subtraction osteotomy (PSO). Minimally invasive surgical (MIS) techniques have been developed for lateral transpsoas anterior column realignment (ACR). Combination of these two techniques for deformity correction has not been previously described. Our objectives were to investigate the cadaveric feasibility of a combined hybrid MIS ACR- PSO for deformity correction and review early clinical experience.

Methods: Feasibility and safety of the combined technique was evaluated in three fresh cadaveric specimens; an ACR with PSO was performed. As an adjunct, 9 clinical cases were treated with this combined approach for global sagittal imbalance. Operative results, estimated blood loss(EBL), complications, early surgical and clinical outcomes were assessed.

Results: In the cadaveric study, the combined approach allowed for significant correction of segmental lordosis (Figure 1). A total of 9 patients were identified (mean age $=65,33 \%$ female) (Figure 2). ACR was most frequently performed at L1-2, and PSO most commonly performed at L3; an average of $11 \pm 3.8$ levels were fused. A significant decrease in pelvic tilt $\left(28.9^{\circ}\right.$ vs. $17.9^{\circ}$, $\mathrm{p}=0.013)$ and spinopelvic mismatch $\left(49.2^{\circ}\right.$ vs. $\left.11.4^{\circ}, \mathrm{p}<0.001\right)$ was observed postoperatively, with a significant increase in lumbar lordosis $\left(-4.4^{\circ}\right.$ vs. $\left.-44.1^{\circ}, \mathrm{p}<0.001\right)$, intradiscal angle $\left(2.1^{\circ}\right.$ vs. $-23.7^{\circ}$, $\mathrm{p}=0.001)$, and thoracic kyphosis $\left(19.7^{\circ}\right.$ vs. $\left.37.6^{\circ}, \mathrm{p}=0.036\right)$. Four patients had 1-year postoperative films available, with sustained correction. Average EBL was $45 \pm 16.4 \mathrm{~mL}$ in the lateral approach, and $3.6 \pm 2.8 \mathrm{~L}$ for the posterior approach. One wound dehiscence occurred, one durotomy, and one postoperative weakness necessitating additional laminectomy occurred with no other significant complications in the perioperative period.

Conclusion: Our early experience suggests that a combined ACR and PSO with posterior fixation approaches allows for significant correction of segmental lordosis and global imbalance in a safe, effective, and complimentary manner. This combined approach may maximize results attainable with hybrid MIS-open surgical techniques. 


\section{Predictive Model for Distal Junctional Kyphosis After Cervical Deformity Surgery}

Peter G Passias, MD; Dennis Vasquez Montes, MS; Gregory W. Poorman; Themistocles Protopsaltis, MD; Samantha R. Horn; Bassel G. Diebo, MD; Cole Bortz, BA; Frank Segreto; Muhammad Burhan Ud Din Janjua, MD; Christopher P. Ames, MD; Justin S. Smith, MD, PhD; Virginie Lafage, PhD; Renaud Lafage; Christopher I. Shaffrey, MD; Shay Bess, MD; Frank Schwab; International Spine Study Group

Introduction: Distal Junctional Kyphosis(DJK) is a primary concern of surgeons correcting cervical deformity. Identifying patients and procedures at higher risk for developing this condition is paramount in improving patient selection and care. The aim of this study was to develop a risk index for DJK development up to two years after surgery.

Methods: Retrospective review of multicenter cervical deformity database. Patients $>18 \mathrm{yr}$ meeting one of the following: C2-7 Cobb angle $>10^{\circ}$, coronal Cobb angle $>10^{\circ}, \mathrm{C} 2-\mathrm{C} 7$ sagittal vertical axis $>4 \mathrm{~cm}$, or chin-brow vertical angle $>250$. DJK definition:clinical diagnosis(by enrolling surgeon) and development of angle $<-10^{\circ}$ from the end of fusion construct to the 2 nd distal vertebra, and change in this angle by $<-10^{\circ}$ from baseline. Conditional Inference Decision Trees identified factors predictive of DJK incidence and the cut-off points at which they have an effect. A conditional Variable-Importance table was constructed and 12 influencing factors were found: binary logistic regression at significant cut-offs indicated effect size.

Results: 101 surgical patients(60.1yrs, $58.3 \% \mathrm{~F}, \mathrm{BMI}: 30.2)$ undergoing long cervical deformity correction(levels fused:7.1, osteotomy: $49.5 \%$, posterior: $46.5 \%$, anterior: $17.8 \%$, combined:35.7\%) were included. In two years after surgery $6 \%$ of patients were diagnosed with clinical DJK, however $23.8 \%$ of patients had radiographic DJK. Patients with neurologic symptoms were at risk for DJK(OR:3.71 CI:0.11-0.63). However, no significant relationship was found between osteoporosis, age, or ambulatory status with DJK incidence. Baseline radiographic malalignments were the most numerous and strong predictors for DJK:[1]C2-T1 Tilt >5.33(OR:6.94,CI:2.99-16.14), [2] Kyphosis <-50.6 (OR:5.89,CI:0.07-0.43), [3]C2-C7 lordosis<$12^{\circ}$ (OR:5.7,CI:0.08-0.41), [4]T1 Slope minus Cervical Lord osis $>36.4(\mathrm{OR}: 5.6, \mathrm{CI}: 2.28-13.57), \quad[5] \mathrm{C} 2-\mathrm{C} 7 \quad \mathrm{SVA}>56.3^{\circ}(\mathrm{OR}$ : 5.4,CI:2.20-13.23), and [6]C4_Tilt >56.7(OR:5.0,CI:1.90-13.1). Clinically, combined approaches(OR:2.67,CI:1.21-5.89) and usage of Smith Petersen osteotomy(OR:2.55,CI:1.02-6.34) were the most important predictors for DJK.

Conclusion: In a surgical cohort of cervical deformity patients, we found a $23.8 \%$ incidence of DJK. Preoperative TS-CL, Cervical Kyphosis, SVA, and Cervical Lordosis all strongly predicted DJK. Knowledge of these factors will potentially help direct future study and strategy aimed at minimizing this potentially dramatic occurrence.

405 Adult Spinal Deformity Patients Demonstrate Impaired Postural Stability and Dynamic Balance Compared to Age-matched Controls

Jakub Godzik, MD, MSc; Chris Frames, BS; Victoria Smith, BS; Markey C Olson, MS; Udaya K. Kakarla, MD; Juan S. Uribe, MD, FAANS; Thurman Lockhart, PhD; Jay D. Turner, MD, PhD

Introduction: Malalignment in adult spinal deformity (ASD) appears to impact body mechanics during gait testing. However, little is known about the influence of ASD on balance and postural stability. Our objective was to assess postural stability in patients with ASD, and evaluate the association with radiographic parameters and quality of life (QOL) metrics.

Methods: Eleven patients with ASD were prospectively enrolled. Inclusion criteria: coronal cobb $(C C)=20^{\circ}$, pelvic incidence lumbar lordosis (PI-LL) $>10^{\circ}$, sagittal vertical axis (SVA) $=5 \mathrm{~cm}$, pelvic tilt $(\mathrm{PT})=25^{\circ}$, and thoracic kyphosis $(\mathrm{TK})=60^{\circ}$. Exclusion criteria: inability to walk/stand, or presence of other confounding neurological conditions. Postural stability was evaluated using a forceplate and computerized dynamic posturography (Neurocom); two tests were selected to assess voluntary static postural control [Sensory Organization Test (SOT)] and dynamic involuntary postural control given a global perturbation (backward translation). Patient performance was compared to age-gender matched controls. QOL metrics included SRS-22, SF-36, and Morse Fall Risk (MFS). Correlations between postural stability and radiographic measurements were performed using spearman's correlation

Results: Mean age was $65 \pm 4.6$, and $6 / 13(46 \%)$ were female. Mean PI-LL was $31.2 \pm 12.7$, PT was $32.8 \pm 8.0$, SVA was $11.8 \pm 6.3$, TK was $27.3 \pm 16.6$, and CC was $14.5 \pm 16.7$. Patients who self reported falling in past 6 months demonstrated significantly lower SOT scores $(\mathrm{p}=0.04)$, and significantly lower SRS self-image subscore $(\mathrm{p}=0.003)$. ASD patients demonstrated significantly lower SOT scores $(\mathrm{p}<0.03)$, and greater latency of limb movement during backward translation $(\mathrm{p}=0.0379)$ compared to age-matched controls. Correlation analysis demonstrated a positive correlation between TK and mediolateral sway (predictor of falls) in the eyes open and eyes closed conditions $(\mathrm{p}<0.04)$.

Conclusion: ASD patients demonstrate impaired postural stability and dynamic balance compared to normal controls. Further, postural stability and QOL metrics correlated with self-reported falls. These findings suggest that ASD patients have altered balance, which predisposes to elevated risk of falls and injury.

406 Rate of Instrumentation and Fusion-related Complications After Surgical Treatment for Severe Pediatric Spinal Deformity within 2 years: A Prospective Multi-center Cohort Study.

Munish C. Gupta, MD; Lawrence Lenke, MD; Jahangir Asghar; Oheneba BOACHIE-ADJEI, MD, DS; Patrick J. Cahill, MD; Mark Alan Erickson, MD, MMM; Sumeet Garg, MD; Peter O. Newton, MD; Amer F. Samdani, MD; Suken A Shah; Harry Shufflebarger, MD; Brenda A Sides, MA; Paul Sponseller, MBA; Daniel Sucato, MD; Michael P. Kelly, MD

Introduction: Severe pediatric deformity can be extremely challenging to treat due to difficulties with instrumentation placement in small patients, stress on implants due to correction of severe deformities, and use of three column osteotomies. This study analyzed the instrumentation and fusion related complications in complex spine deformity surgical cases.

Methods: 176 patients with severe complex spinal deformity ( $>100^{\circ}$ or planned VCR) were included from a prospective database with a minimum 2 year follow-up. Complications with or without revision due to pseudoarthrosis, instrumentation failure, infection requiring instrumentation removal and progression of deformity were all analyzed.

Results: 176 patients out of 313 pts reached a minimum of 2 years follow up. 21 patients $(12 \%)$ had complications associated with the instrumentation. 15 patients $(9 \%)$ required 16 revision surgeries because of instrumentation failure. 6 patients $(3 \%)$ had complications but did not require any revisions. The 16 revision surgeries included 7 (43\%) with loss of fixation. The average time for the revision surgery was 13 months (0-28) after the index surgery. Only one patient had 2 revisions. 4 patients were revised 
for pseudarthrosis at an average of 23 months (17-35). 2 patients $(13 \%)$ had revisions for prominent instrumentation (both at 27 mos post), 2 (13\%) for infection (19 and 36 months respectively), and one patient had revision surgery for deformity progression at 2 months postoperatively. The patients that did not have revision surgeries included 2 with prominent implants both found at 18 months postop, 2 with progressive deformity/PJK at 18 months average (14-22), and 2 had loss of fixation at 6 months (0-12).

Conclusion: Pediatric patients with severe spinal deformity are high risk for revision surgeries at $12 \%$ rate within 2 years. The average time for revision surgery was 19 months postoperatively. These patients require close follow-up and will require continued follow-up after 2 years.

407 Comparison of Segmental Lordosis and Global Spinopelvic Alignment After Single-Level Lateral Lumbar Interbody Fusion or Transforaminal Lateral Interbody Fusion

Yamaan S. Saadeh, MD; Jacob R. Joseph, MD; Brandon W Smith, MD, MSCR; Michael Kirsch; Amr M Sabbagh, BS; Paul Park, MD

Introduction: Lateral lumbar interbody fusion (LLIF) has experienced a steady increase in popularity since its introduction. LLIF has been thought to allow for greater improvement in lordosis than transforaminal lumbar interbody fusion (TLIF). However, there is very limited direct comparison data on the degree of regional and global alignment change after single-level LLIF and TLIF procedures.

Methods: A retrospective analysis of patients who underwent LLIF or TLIF for lumbar degenerative disease at a single institution was performed. Twenty patients who underwent single level LLIF were matched to 20 patients who underwent single level TLIF by gender and level of interbody fusion. All included patients had preoperative and postoperative standing scoliosis $\mathrm{x}$-rays. Changes in segmental lordosis (SL) at the fused level, lumbar lordosis (LL), sagittal vertical axis (SVA), and pelvic incidence-lumbar lordosis mismatch (PI-LL) were measured. Statistical analysis was performed using unpaired t-tests. Means were reported with standard error.

Results: Within each group, 2 patients had cages placed at L2-3, 4 patients had cages placed at L3-4, and 14 patients had cages placed at L4-5. The LLIF group demonstrated a statistically significantly increased change in degrees of segmental lordosis compared to the TLIF group $\left(+4.9^{\circ} \pm 0.7 \mathrm{vs}+2.6^{\circ} \pm 0.4, \mathrm{P}=0.004\right)$. There were no statistically significant changes in global spinopelvic parameters in the LLIF versus the TLIF group, LL $\left(+2.15^{\circ}\right.$ \pm 0.8 vs $\left.+2.6^{\circ} \pm 0.6, \mathrm{P}=0.06\right)$, change in PI-LL $\left(-1.05^{\circ} \pm 1.0\right.$ vs $\left.-2.15^{\circ} \pm 0.7, \mathrm{P}=0.38\right)$, and change in SVA $(-2.2 \mathrm{~cm} \pm 0.8$ vs -1.51 $\mathrm{cm} \pm 0.5, \mathrm{P}=0.48)$, respectively.

Conclusion: LLIF achieved greater improvements in segmental lordosis than TLIF. However, regionally and globally, there were no significant differences with either procedure after a single level intervention. The increased lordosis from LLIF compared to TLIF may be more impactful globally in multi-level fusions.

408 Death Within One Year After Adult Spinal Deformity Surgery: Incidence and Predictors

Scott L. Zuckerman, MD; Nikita Lakomkin, BA; Justin S. Smith, MD, PhD; Christopher I. Shaffrey, MD, FACS; Clinton J. Devin, MD

Introduction: Surgery for adult spinal deformity (ASD) can significantly improve quality of life yet morbidity is high. The most devastating complication after these elective operations is death. Our objective was to report the incidence, preoperative factors, and postoperative complications associated with death within 1 year following ASD surgery.

Methods: Adults who underwent thoracolumbar spinal deformity correction between 2008 and 2014 were identified in the National Surgical Quality Improvement Program (NSQIP) database. Demographic characteristics were extracted. The primary outcome was death within one year of ASD surgery. Propensity score matching was used to control for confounding factors, followed by univariate/multivariable logistic regression to predict the odds of death within one year of ASD surgery.

Results: A total of 6,158 patients underwent ASD surgery and $61(1.00 \%)$ died within one year of surgery. Controlling for age, gender, ASA score, and postoperative complications, four preoperative factors were associated with death: age (OR 1.03, 95\% CI 1.01, 1.06, $\mathrm{p}=0.012$ ), ASA score (OR 4.32, 95\% CI 2.68, 6.94, p<0.001), cancer history (OR 7.91, 95\%CI 4.23, 14.78, $\mathrm{p}<0.001)$ and unintentional weight loss (OR 4.65, 95\%CI 1.68, $12.89, \mathrm{p}=0.003)$. Using propensity score matching and multivariable logistic regression, three postoperative complications were associated with death: pneumonia (OR 4.00, 95\%CI 1.68, 9.53), DVT (OR 12.76, 95\%CI 1.20, 8.10) and unplanned intubation (OR $3.13,95 \%$ CI 1.15, 8.50).

Conclusion: Death after elective ASD surgery is a devastating yet rare event with an incidence of $1.00 \%$. Preoperative factors of age, ASA score, cancer history, and unexpected weight loss, along with postoperative complications of pneumonia, DVT, and unplanned intubation were independently associated with death within one year of ASD surgery. Interestingly, the more severe complications of sepsis, PE, and MI did not independently predict death.

409 Predictive Analytics for Determining Extended Operative Time in Corrective ASD Surgery

Peter G Passias, MD; Gregory W, Poorman; Dennis VasquezMontes; Gregory M. Mundis, MD; Neel Anand, MD; Samantha R. Horn; Bassel G. Diebo, MD; Cole Bortz, BA; Frank Segreto; Muhammad Burhan Ud Din Janjua, MD; Douglas C. Burton, MD; Aaron James Buckland, MBBS, FRAC

Introduction: More sophisticated techniques for surgical correction of adult spinal deformity (ASD) have increased op-times, which are correlated with higher complication incidence. This study employs a statistical learning algorithm to determine key variables affecting op-time.

Methods: Retrospective review of prospective multi-center database, cases between 2008-2016 as part of the International Spine Study Group. Conditional Inference Decision Trees assessed factors affecting skin-to-skin op-time and the cut-off points at which factors have a global effect. A Conditional VariableImportance table was constructed based on a non-replacement sampling set of 2000 Conditional Inference trees. Following identification of the top 15 influencing factors, means comparison for each variable at significant cut-offs indicated variable effect size.

Results: 544 surgical patients undergoing ASD-corrective surgery(58.0yrs, mean 11.3 levels fused, mean op-time:377.6 min) were included. The strongest predictor for op-time was institution/ surgeon. Grouped by decision tree hierarchy, center/surgeons a and $\mathrm{b}$ were on average 2 hours faster than center/surgeons $\mathrm{c}-\mathrm{f}$, which were 43 minutes faster than centers g-j(all $p<0.001)$. Other key predictors were, in order, approach (combined vs. posterior increases time by 139 minutes, $<<0.001)$, number of levels fused ( $<4$ vs. $5-9$ increased time by 68 minutes, $p<0.050 ; 5-9$ vs. $>10$ increased time by 47 minutes, $\mathrm{p}<0.001)$, age (age $>50$ increases time by 57 minutes, $\mathrm{p}<0.001$ ), and patient frailty(score $>1.54$ increases 
time by 65 minutes,p<0.001). Surgical techniques, including three-column osteotomies(35 minutes), interbody device(45 minutes), and decompression(48 minutes), also increased op-time. Minor and major complications were correlated with $>66$ minutes of increased op-time. Increased op-time also correlated with increased LOS, EBL, and inferior 2-year ODI scores.

Conclusion: Procedure location and specific surgeon are key factors determining op-time accounting for operative time increases of more than two hours. Surgical approach and number of levels fused were next most significant in elongating surgeries, respectively. Extended op-time correlated with longer length of hospital stay, higher EBL, and inferior 2-year improvement in ODI.

410 High Rate of Intraoperative Monitoring (IOM) Alerts in 176 Severe Pediatric Deformity Patients and its Relationship to the Deformity Angularity Ratio (DAR).

Munish C. Gupta, MD; Lawrence Lenke, MD; Jahangir Asghar; Oheneba BOACHIE-ADJEI, MD, DS; Patrick J. Cahill, MD; Mark Alan Erickson, MD, MMM; Sumeet Garg, MD; Peter O. Newton, MD; Amer F. Samdani, MD; Suken A Shah; Harry Shufflebarger, MD; Brenda A Sides, MA; Paul Sponseller, MBA; Daniel Sucato, MD; Elizabeth L. Yanik, PhD, ScM; Michael P. Kelly, MD

Introduction: Severe pediatric deformity is challenging with higher complications. The surgical procedures were analyzed in terms of neurologic safety.

Methods: Patients with severe spinal deformity (minimum curve of $100^{\circ}$ or a planned VCR) underwent operative treatment for their deformity and were followed for minimum 2 years. Logistic regression was used to evaluate associations of different procedures and radiographic parameters (VCR procedure, ant/post procedure, coronal C-DAR, sagittal S-DAR) with intraoperative neural monitoring alerts (SSEP, TCeMEP, and any IOM) and postoperative deficits.

Results: $176 / 313$ enrolled in the study met the inclusion criteria; we excluded patients with $<2$ yrs FU. 76/176 (43\%) patients had a VCR procedure and one patient had a PSO. $162(92 \%)$ had a posterior only approach; $14(8 \%)$ were treated with a combined ant/post-surgery. 75 patients had 114 total intraop monitoring alerts. S-DAR was associated with any intraop alerts $(p=0.04)$ and TCeMEP $(p=0.04)$. C-DAR was associated with SSEP alerts $(\mathrm{p}=0.02)$. The 5 most common triggering events were correction maneuvers, 3-column osteotomy, implant and instrumentation placement, and hypotension. Some patients had multiple triggering events $(\mathrm{N}=26)$. 161 were neurologically normal preop. 150 pts remained normal neurologically postop and 11 had new deficits. However at 2 years postop, only $1 / 11$ still had a deficit. $14 \mathrm{pts}$ had a neurologic deficit preop. Postop 4 pts improved to a normal neurologic status, 9 pts continued to have a deficit, and 1 pt had partial recovery neurologically. At 2 years, out of the 14 neurologically abnormal pts preoperatively, 11 totally recovered, 2 partially recovered and 1 deficit did not improve.

Conclusion: Severe deformity pediatric patients have a high incidence of intraoperative neural monitoring alerts (43\%); however, only 1 new permanent deficit occurred. 13 of 14 patients improved or recovered from preoperative neurologic deficits. Sagittal DAR is associated with IOM alerts. Neural monitoring should be mandatory in these cases.

411 How Important is the Anterior Column Support in a Long Lumbopelvic Spinal Fixation? An In-silico Biomechanics Analysis

Woojin Cho, MD, PhD; Wenhai Wang, $\mathrm{PhD}$; Brandon Bucklen, PhD; Howard B Whitman, BA
Introduction: The addition of anterior column support at the bottom of a long construct has been proposed to support the lumbosacral junction and potentially prevent posterior lumbopelvic fixation failure. However, a retrospective study has shown that adding anterior column support was not sensitive to construct failure, highlighting the possibility that posterior fusion quality may be a more important factor than previously thought.1

Methods: Finite element models were created to match the average spinal-pelvic parameters of two patient cohorts [major failure and non-failure (Figure 1)] reported in the literature. Gravimetric loading was applied to simulate a standing position. Posterior solid fusion was simulated by applying spring elements attached to contiguous vertebrae. Anterior column support was simulated by assigning cancellous bone properties to the L4-S1 disc.

Results: Upper body weight acting on the long instrumented spine resulted in 18\% higher translation, $14 \%$ higher rotation, and $20 \%$ higher stress in the major failure group than in the non-failure group. Solid posterior fusion decreased translation and rotation, and also alleviated rod stress in the lower lumbar region (Figures 2 and 3). In this case, anterior support did not change the stress pattern. Conversely, simulated pseudoarthrosis produced stress patterns similar to those of the construct-only spine. In this case, adding anterior support dramatically alleviated stress at the index levels.

Conclusion: The spinopelvic parameters of the major-failure group produced increased gravity load, resulting in increased stresses in comparison to the non-failure group. Simulated posterior "solid" fusion in the lumbar region helped reduce stresses in both major failure and non-failure patients. However, in cases with a simulated pseudoarthrosis, rod stresses were amplified by major failure spinal alignment. In this case, anterior column support was a major factor in reducing rod stress, and should be considered for patients with poor alignment.

412 Patient History of Anemia is an Independent Predictor of 30-Day Readmission in Elderly (e"60 Years Old) Spine Deformity Patients After Elective Spinal Fusion

Aladine A. Elsamadicy, BE; Owoicho Adogwa, M.D., M.P.H; Victoria D Vuong; Amanda Sergesketter; Michael Ongele, B.S.; Joseph S. Cheng, MD, MS; Carlos Antonio Bagley, MD; Isaac O. Karikari, MD

Introduction: Unplanned 30-day hospital readmission rates have is being used as a proxy for quality of care. The aim of this study is to determine if a pre-operative history of anemia is an independent risk factor for readmission within 30 days of discharge.

Methods: The medical records of 697 elderly (=60 years old) deformity patients undergoing elective spinal fusion at a major academic institution from 2005 to 2015 were reviewed. We identified $61(8.9 \%)$ patients with a history of anemia and 636(91.1\%) without (Anemia: $n=61$; No-Anemia: $n=636$ ). Patient demographics, comorbidities, intra- and 30-day post-operative complication and readmission rates were collected for each patient. Primary outcome investigated in this study was unplanned hospital readmission for any reason within 30-days of discharge. The association between pre-operative anemia and 30-day readmission rate was assessed via multivariate nominal logistic regression analysis.

Results: Patient demographics and comorbidities were similar between both groups, with a difference in BMI (Anemia: 27.1 \pm 5.8 $\mathrm{kg} / \mathrm{m} 2$ vs. No-Anemia: $28.2 \pm 6.2 \mathrm{~kg} / \mathrm{m} 2, \mathrm{p}=0.04$ ). Median number of fusion levels and operative time were similar between the cohorts. Anemia cohort had a significantly greater incidence of 
intraoperative durotomy, compared to the No-Anemia cohort (14.8 vs. 5.8, $\mathrm{p}=0.007$ ). Both cohorts had similar postoperative complications and length of hospital stay. There was significant difference in 30-day readmission between the cohorts, with the Anemia cohort experiencing a 2-fold increase (Anemia: $21.3 \%$ vs. $11.0 \%, \mathrm{p}=0.02$ ). Compared the No-Anemia cohort, the Anemia cohort experienced significantly greater incidences of 30-day wound dehiscence (6.6 vs. $1.4, \mathrm{p}=0.005)$ and altered mental status ( 8.2 vs. $1.7, \mathrm{p}=0.001)$. Adjusting for other risk factors, the multivariate nominal logistic regression demonstrates that preoperative anemia is an independent predictor of 30-day readmission after elective spine surgery in the elderly [OR: 2.53 , CI $(1.26,5.07), \mathrm{p}=0.01)$.

Conclusion: Pre-operative anemia is an independent risk factor for readmission within 30-days of discharge after elective spine surgery in elderly patients.

413 Interdisciplinary are Model Decreases Use of Critical Care Services After Corrective Surgery for Adult Degenerative Scoliosis

Owoicho Adogwa, M.D., M.P.H; Aladine A. Elsamadicy, BE; Amanda Sergesketter; Michael Ongele, B.S.; Tarnasky Aaron, B.S.; Victoria D Vuong; Syed Ibad Khalid; Jessica Rose Moreno, RN, BSN; Joseph S. Cheng, MD, MS; Isaac O. Karikari, MD; Carlos Antonio Bagley, MD

Introduction: The aim of this study is to determine whether and interdisciplinary team approach mitigates use of ICU resources.

Methods: A unique model of co-management for elderly patients undergoing lumbar fusion surgery was implemented at a major academic medical center. The Peri-operative Optimization of Senior Health Program (POSH) was launched with the aim of improving outcomes in elderly patients ( $>65$ years-old) undergoing complex lumbar spine surgery. In this model, a geriatrician evaluates elderly patients pre-operatively, in addition to the routine pre-operative anesthesia surgical screening, and co-manages them daily throughout the hospital course of their hospital stay to manage medical comorbid conditions and coordinate multidisciplinary rehabilitation, along with the neurosurgical team. We retrospectively review the first 100 cases after the initiation of the POSH protocol and compared them with the immediately preceding 25 cases to assess the incidence of peri-operative complications and clinical outcomes. The rates of ICU transfer primary as well as the independent predictors of ICU admission are the primary outcomes of this study.

Results: 125 undergoing lumbar decompression and fusion surgery were enrolled in this pilot program. Baseline characteristics were similar between both cohorts. Intra-operative variables as well as number of fusion levels and duration of surgery were similar between both cohorts. There was a significant difference in the use of ICU services(ICU admission rates) between both cohorts, with Non-POSH cohort having a 3-fold increase compared to the POSH cohort $(\mathrm{p}<0.0001)$. In a multivariate binary logistic regression model, lack of an interdisciplinary co-management team approach was an independent predictor for ICU transfers in elderly patients undergoing corrective surgery for adult degenerative scoliosis [OR: $8.51,95 \% \mathrm{CI}(2.972,24.37), \mathrm{p}<0.0001]$.

Conclusion: Our study suggests that an interdisciplinary comanagement model between geriatrics and neurosurgery significantly reduces the use of critical care services. Furthermore, not having co-management model was an independent predictor of need for critical care services.
414 Revision Surgery After T1 versus T2 as Lowest Instrumented Vertebra in Multilevel Posterior Cervicothoracic Fusion: A Multicenter Study

Rafael De la Garza Ramos, MD; Seba Ramhmdani, M.D.; Jonathan P Nakhla, MD; Marc E Comair; Merritt Drew Kinon, MD; Timothy F. Witham, BS, MD; Daniel M. Sciubba, MD; Reza Yassari, MD; Ali Bydon, MD

Introduction: There is a paucity of literature regarding which thoracic level should be selected as lowest instrumented vertebra (LIV) in multi-segmental cervicothoracic fusion procedures, and whether this makes an impact on rates of adjacent segment disease or instrumentation failure. Thus, the purpose of this study is to identify the rate of revision surgery after selecting T1 versus T2 as LIV in multilevel posterior cervicothoracic fusion procedures.

Methods: A retrospective review of two institutional neurosurgical spine operative databases (Montefiore Medical Center and Johns Hopkins Hospital) from 2006 to 2016 was conducted. Adult patients ( $>18$ years of age) who underwent multilevel cervicothoracic fusion procedures (3 or more levels) with either T1 or T2 as LIV were identified. The primary outcome measure was need for revision surgery for wound infection or instrumentation failure. Predictors of revision surgery were identified via logistic regression analysis.

Results: There were 179 patients who met inclusion criteria and were included in our study; 34 in the T1 group (19.0\%) and 145 in the T2 group (81.0\%) with mean follow-up of 24 months (range: 1 92). The rate of revision surgery was $14.7 \%$ in patients with T1 as LIV and $15.2 \%$ in patients with T2 as LIV ( $\mathrm{p}=0.945$, chi-squared test). Revision surgery for instrumentation failure occurred in $8.8 \%$ of cases in the $\mathrm{T} 1$ group and $2.8 \%$ in the $\mathrm{T} 2$ group $(\mathrm{p}=0.127)$. On the other hand, reoperation for wound infection was $5.9 \%$ and $11.7 \%$ for patients in the $\mathrm{T} 1$ and $\mathrm{T} 2$ groups, respectively $(\mathrm{p}=0.320)$. No preoperative or operative parameters were found to be significantly associated with revision surgery after logistic regression analysis.

Conclusion: Patients who underwent multisegmental cervicothoracic fusion procedures down to T1 or T2 showed similar revision surgery rates in this study. Selecting the first versus second thoracic vertebra as lowest instrumented level may not impact the risk of reoperation.

415 2-Year SRS-22r and ODI Patient-reported Outcomes Accurately Correlate with 5-Year Patient-reported Outcomes After Complex Spinal Fusion: A 5-Year Single Institutional Study of 118 Patients

Owoicho Adogwa, M.D., M.P.H; Isaac O. Karikari, MD; Aladine A. Elsamadicy, BE; Amanda Sergesketter; Diego Galan, B.S.; Keith Bridwell, MD

Introduction: Patient-reported outcomes (PROs) are often measured up to 2 years after surgery; however, prospective collection of longitudinal outcomes for 5 years postoperatively can be challenging due to lack of patient follow-up. The aim of this study is to determine whether PROs collected at 2-year follow-up accurately predict long-term PROs 5 years after complex spinal fusion (=5 levels).

Methods: This was an ambispective study of 118 adult patients $(=18$ years old) undergoing $=5$ levels spinal arthrodesis to the sacrum with iliac fixation from January 2002 to December 2011. Patient demographics, radiographic parameters, as well as intraoperative variables were collected. PRO instruments (Scoliosis Research Society [SRS]-22r function, self-image, mental health, pain, and Oswestry Disability Index [ODI]) were com- 
pleted before surgery then at 2- and 5-years after surgery. Primary outcome investigated in this study was the correlation between SRS-22r domains and ODI collected at 2- and 5-year follow-up.

Results: Of the 118 patients( $28 \%$ primary and $72 \%$ revision), $51.7 \%$ underwent pedicle subtraction osteotomies (PSO). The average pre- and post-operative major coronal curve Cobb angle for the cohort was $32.1 \pm 23.7^{\circ}$ and $19.8 \pm 19.3^{\circ}$, respectively. There was a strong correlation between 2- and 5-year ODI and between 2- and 5-year SRS-22r domains, including function, self-image, mental health, and pain. Of the PROs, ODI showed the greatest absolute change from baseline to 2- and 5- year follow-up (2-year ? 17.6 \pm 15.9 ; 5-year ? 16.5 \pm 19.9 ) followed by SRS-22r self-image (2-year ? 1.4 \pm 0.96 ; 5-year ? 1.3 \pm 1.0 ), pain (2-year ? $0.94 \pm 0.97$; 5-year ? $0.80 \pm 1.0$ ), function (2-year ? $0.60 \pm 0.62$;

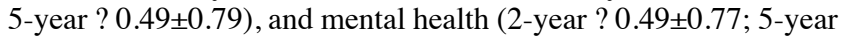
? $0.38 \pm 0.84)$.

Conclusion: Patient-reported outcomes collected at 2-years follow-up may accurately predict long-term PROs (5-year followup).

416 Continuous Activity Tracking Using a Wrist-mounted Device in Adult Spinal Deformity: Proof of Concept

Jakub Godzik, MD, MSc; Tyler Scott Cole, MD; Corey Tyler Walker, MD; Alex Whiting, MD; Udaya K. Kakarla, MD; Juan S. Uribe, MD, FAANS; Jay D. Turner, MD, PhD

Introduction: Sagittal imbalance in adult spinal deformity (ASD) likely influences balance and ambulatory capacity due to muscular demand, pain and disability. Disability measures for ASD rely heavily on ambulatory function, yet current metrics may fail to fully capture the contribution. We sought to determine (1) the utility of continuous remote step-count monitoring and activity tracking in ASD using a consumer-friendly accelerometer, and (2) investigate trends and patterns following surgical intervention.

Methods: One patient with progressive deformity was enrolled in the current study. Daily step count (SC), distance travelled (DT), and levels of activity [sedentary(SA), light activity (LA)] were measured using a wrist-mounted device (FitBit) for 17 months. Preoperative period was separated into months (-9 to -1$)$; postoperative period was categorized ("immediate postoperative," "2-4 months," "5-6 months," "7-8 months"). Spinopelvic parameters were measured from standing radiographs. Differences were assessed using ANOVA, statistical significance was $\mathrm{p}<0.05$.

Results: Patient presented with progressive deformity (coronal cobb(CC): 61; sagittal vertical axis (SVA): $15.7 \mathrm{~cm}$; coronal vertical axis (CVA): $11 \mathrm{~cm})$, and underwent T4-ilium fusion for correction of deformity (CC: 8; SVA: 4cm; CVA: 2cm) (Figure 1 ); recovery period was uneventful. The device was worn $216 / 520$ $(41 \%)$ of recorded days, with an average SC of $5254 \pm 2696$ per day. SC $(\mathrm{p}<0.001)$, DT $(\mathrm{p}<0.001)$, and minutes of LA $(\mathrm{p}<0.001)$ declined over the 9-month preoperative course, while SA increased $(\mathrm{p}<0.001)$ (Figure 2). Following surgery, SC, DT, and LA decreased in the early postoperative period, with a significant increase at 7-8 months compared to the preoperative period $(\mathrm{p}<0.001$; Figure 3,4$)$.

Conclusion: Wrist-mounted devices are a non-invasive and effective measure to track daily activity levels and ambulatory capacity of spinal deformity patients. In a single case, progressive deformity correlated with worsening activity levels, while deformity correction improved daily step count and activity level. These results support remote step count monitoring as an exploratory outcome for future studies.
417 Retrospective Multicenter Assessment of Risk Factors for Rod Fracture Following Minimally Invasive Surgery for Adult Spinal Deformity Correction

Jakub Godzik, MD, MSc; Nima Alan, MD; Randall Hlubek, MD; Konrad Bach, MD; Gregory M. Mundis, MD; Adam S. Kanter, MD; David O. Okonkwo, MD, PhD; Jay D. Turner, MD, $\mathrm{PhD}$; Juan S. Uribe, MD, FAANS

Introduction: Surgical correction of sagittal imbalance in adult spinal deformity (ASD) traditionally involves three column osteotomies $(3 \mathrm{CO})$, however these can be associated with significant morbidity and elevated rate of rod fracture (RF). The lateral transpsoas anterior column realignment (ACR) was developed as a less invasive procedure for restoring lordosis. The objective of this study was to retrospectively assess the rates and risk factors for RF following ACR in deformity correction surgery.

Methods: A retrospective multicenter review of all patients with ASD undergoing ACR for deformity correction. ASD was defined as coronal $\operatorname{cobb}(\mathrm{CC})=20^{\circ}$, pelvic incidence lumbar lordosis $(\mathrm{PI}-\mathrm{LL})>10^{\circ}$, sagittal vertical axis $(\mathrm{SVA})=5 \mathrm{~cm}$, pelvic $\operatorname{tilt}(\mathrm{PT})=25^{\circ}$, and thoracic kyphosis $(\mathrm{TK})=60^{\circ}$. Inclusion criteria were ASD, age $>18$, use of ACR, baseline standing radiographs, and development of RF or full radiographs atleast 1-year after surgery that did not demonstrate RF.

Results: 90 patients met inclusion criteria with mean followup of $2.3 \pm 1.4$ years (mean age $64.1 \pm 9.4,60 \%$ female). Mean preoperative CC was $21.5 \pm 16.2^{\circ}$, SVA $9.2 \pm 5.4 \mathrm{~cm}$, PT $28.2 \pm 10.7^{\circ}$, with PI-LL $32 \pm 16^{\circ}$. Most common ACR location was L3/4 (47\%), with mean intradiscal angle of $20 \pm 9.1^{\circ}$. Mean fusion length was $7.5 \pm 3.6$ levels. Four patients $(4 / 90,4.4 \%)$ developed RF within 6-12 months after initial surgery. RF occurred proximal and adjacent to ACR in majority of cases; RF was not associated with location of ACR $(p=0.81)$, cage size $(p=0.94)$, focal correction $(p=0.49)$, posterior osteotomy $(\mathrm{p}=0.24)$, rod material $(\mathrm{p}=0.8)$, degree of deformity correction $(\mathrm{p}>0.07)$, or an interbody at $\mathrm{L} 5 / \mathrm{S} 1(\mathrm{p}=0.06)$. RF was associated with longer fusion constructs (7.2 vs. 13.6 levels, $\mathrm{p}=0.002$ ) in univariate and multivariate analyses.

Conclusion: Rod fracture occurred in $4.4 \%$ of ASD patients undergoing ACR with a minimum of 1-year follow-up. Rod fracture was not related to focal correction, but rather global correction and extent of fusion. Future studies are needed to compare RF rates between $\mathrm{ACR}$ and $3 \mathrm{CO}$ techniques.

418 Alignment Risk Factors for Proximal Junctional Kyphosis and the Effect of Lower Thoracic Junctional Tethers for Adult Spinal Deformity

Thomas Buell, MD; John C Quinn, MD; Avery Lee Buchholz, MD; James Nguyen, MD, BA; Marcus D. Mazur, MD; Shay Bess, MD; Breton G. Line, BSME; Christopher P. Ames, MD; Frank Schwab, MD, PhD; Virginie Lafage, PhD; Christopher I. Shaffrey, MD, FACS; Justin S. Smith, MD

Introduction: Proximal junctional kyphosis (PJK) may occur more frequently in the lower thoracic spine (LT) after multilevel fusion surgery for adult spinal deformity (ASD). The objectives of this study were to (1) determine the effect of junctional tethers on uppermost instrumented vertebrae (UIV) alignment for ASD patients with LT UIV (2) identify new alignment risk factors for PJK in this subset.

Methods: Retrospective analyses of ASD patients who underwent posterior instrumented fusion with LT UIV. Uni- and multivariate analyses were performed on demographic, surgical (including use of tethers), and radiographic parameters. PJK was defined as proximal junctional angle $>10^{\circ}$ and $>10^{\circ}$ greater than 
the corresponding preoperative measurement. Tethers were placed using one of two techniques: polyethylene tape only or tape with crosslink. We measured the angle from the UIV inferior endplate to horizontal (UIV angle) to evaluate the effect of tethers on UIV alignment.

Results: 120 (89\%) of 135 consecutive patients achieved minimum 1-year follow-up (mean=28 months [range:12-57 months]; mean age $=67.3$ years; $64.2 \%$ female), of whom 73 had tethers and 49 developed PJK. PJK was less common in patients who had tethers than those who did not (23/73 [32\%] versus 26/47 [55\%], $\mathrm{p}=0.010)$. On multivariate analysis, use of tethers remained a significant predictor for avoiding PJK (OR $=0.063$, 95\% CI 0.016 $0.247, \mathrm{p}<0.001)$. Additionally, PJK in the tether group was more common when there was greater postoperative lordosis of the upper lumbar spine $(\mathrm{p}=0.047)$ and greater UIV angle $(\mathrm{p}=0.026)$. There was a nonsignificant trend toward lower revision rate for PJK when comparing tether and non-tether cohorts $(5 \%$ versus $9 \%, \mathrm{p}=0.516)$.

Conclusion: Tethers significantly reduced PJK in ASD patients with LT UIV. In tethered patients, PJK was more common with greater postoperative lordosis of the upper lumbar spine and greater UIV angle. This suggests potential benefit of tethers to mitigate the effects of UIV malalignment.

419 A Pilot Study on Posterior Polyethylene Tethers to Prevent Proximal Junctional Kyphosis After Multilevel Spinal Instrumentation for Adult Spinal Deformity.

Thomas Buell, MD; Avery Lee Buchholz, MD; John C Quinn, MD; Shay Bess, MD; Breton G. Line, BSME; Christopher P. Ames, MD; Frank Schwab, MD, PhD; Virginie Lafage, $\mathrm{PhD}$; Christopher I. Shaffrey, MD, FACS; Justin S. Smith, MD, PhD

Introduction: Proximal junctional kyphosis (PJK) is a common problem after multilevel spine instrumentation. The objective of this study is to determine if junctional tethers reduce PJK after multilevel instrumented surgery for adult spinal deformity (ASD).

Methods: ASD patients who underwent posterior instrumented fusion were divided into 3 groups: no tether (NT), polyethylene tape only (TO; passed through base of UIV +1 and UIV-2 and tied securely), and tape with crosslink (TC; passed through base of $\mathrm{UIV}+1$ and tied to crosslink between UIV-2 and UIV-3). PJK was defined as proximal junctional angle $>10^{\circ}$ and $>10^{\circ}$ greater than the corresponding preoperative measurement.

Results: 184 (96\%) of 191 consecutive patients achieved minimum 3-month follow-up (vast majority of PJK occurs within 6 weeks of surgery). Mean follow-up was 20 months (range:356 months), mean age was 66 years, and $67.4 \%$ of patients were women. There were no significant differences between groups based on age, BMI, number of instrumented vertebrae, pelvic fixation, 3-column osteotomy, combined anterior-posterior approach, and sagittal radiographic parameters. On multivariate analysis, older age and greater change in lumbar lordosis were independent predictors of PJK. Junctional tethers were a significant predictor to avoid PJK. PJK rates were $45.3 \%(29 / 64), 34.4 \%(22 / 64)$, and $17.9 \%(10 / 56)$ for NT, TO, and TC, respectively. PJK rate for TC was significantly lower than NT $(\mathrm{p}=0.001$; OR=3.81). KaplanMeier analysis showed significant time-dependent reduction in PJK for TC versus NT (log rank test, $\mathrm{p}=0.010)$.

Conclusion: Junctional tethers significantly reduced occurrence of PJK. This difference was progressive from NT to TO to $\mathrm{TC}$, but only reached significance for NT versus TC. This suggests potential benefit of tethers to reduce PJK, and that future prospective studies with longer-term follow-up are warranted.

\section{Risk Factors for Pseudarthrosis in Pediatric Neuromuscular Scoliosis}

Brandon J Toll, BA; Muhammad Burhan Ud Din Janjua, MD; Amer F. Samdani, MD; Joshua M. Pahys, MD; Steven Wei-Hung Hwang, MD

Introduction: Pseudarthrosis is a common cause of reoperation for patients who underwent surgical deformity correction for scoliosis. As such, this study endeavors to identify and characterize risk factors associated with the incidence of pseudarthrosis in pediatric neuromuscular scoliosis.

Methods: Data were retrospectively collected from a singlecenter cohort of 60 consecutive patients receiving spinal fusion for neuromuscular scoliosis between January 2008 and December 2016 with a minimum of two years follow-up. Univariate and multivariate regression analyses, as well as binary correlational models and student's t-tests, were employed for further statistical analysis.

Results: The present cohort consisted of 29 males and 31 females with a mean age at surgery of 14 years $( \pm 2.7 \mathrm{SD}$, range 7.5-19.5). The most prevalent diagnoses were SCI $(25.0 \%), \mathrm{CP}$ $(21.7 \%)$, and neurofibromatosis $(11.7 \%)$. Analysis reflected an overall pseudarthrosis rate of $8.3 \%$. Cases were reliably predicted by primary diagnosis of SCI $(\mathrm{p}=0.005)$, greater pelvic obliquity at follow-up $(\mathrm{p}=0.047)$, and smaller primary curve magnitude at follow-up $(\mathrm{p}=0.045)$. Furthermore, there was a significant inverse association with patient height at follow-up $(\mathrm{p}=0.049)$, preoperative weight $(\mathrm{p}=0.018)$.

Conclusion: Our results suggest that within the pediatric sub-population of neuromuscular scoliosis, pseudarthrosis is most prevalent among patients with spinal cord injuries. Scoliotic curves lesser in magnitude and in shorter patients also appear at significant risk for pseudarthrosis, possibly resulting from initial surgical overcorrection and increased kyphotic deformity, respectively. The observed inverse associations with height and weight may additionally reflect individual differences in skeletal maturity, poor nutritional status, and compromised bone quality.

421 Outcomes After Posterior Spinal Fusion in Pediatric Neuromuscular Scoliosis: A Retrospective Analysis of Perioperative Complications \& Risk-factors

Brandon J Toll, BA; Muhammad Burhan Ud Din Janjua, MD; Amer F. Samdani, MD; Joshua M. Pahys, MD; Steven Wei-Hung Hwang, MD

Introduction: Current literature demonstrates high rates of perioperative complications subsequent to spinal fusion procedures intended to address neuromuscular scoliosis. The current study aims to evaluate complications associated with surgical correction of neuromuscular scoliosis and characterize potential risk factors.

Methods: Data were retrospectively collected from a singlecenter cohort of 102 consecutive patients receiving spinal fusions for neuromuscular scoliosis between January 2008 and December 2016 with a minimum of 6 months follow-up. These cases were further stratified into an additional sub-cohort of patients whose records included a minimum of two years follow-up data as well. Major and minor complications were identified and classified into sub-categories consistent with current literature. Univariate and multivariate regression analyses, as well as binary correlational models and student's t-tests, were employed for further statistical analysis.

Results: The present cohort represented 53 males and 49 females with a mean age at surgery of 14.0 years $( \pm 2.7 \mathrm{SD}$, 
range 7.5-19.5). The most prevalent diagnoses were $\mathrm{CP}(26.5 \%)$, SCI $(24.5 \%)$, and neurofibromatosis $(10.8 \%)$. Analysis reflected an overall complication rate of $27 \%$ (37 complications in 27 patients), $81.1 \%$ of which constituted major complications $(n=30)$ compared to $18.9 \%$ minor complications $(n=7)$. Complications as a whole were reliably predicted by increased intraoperative blood loss $(p=0.012)$, increased intraoperative time $(p=0.046)$, non-ambulatory status $(\mathrm{p}=0.037)$, greater pelvic obliquity at follow-up $(\mathrm{p}=0.028)$, and greater magnitude of sagittal profile at follow-up $(\mathrm{p}=0.048)$. Pulmonary comorbidity $(\mathrm{p}=0.001)$, increase in weight $(\mathrm{p}=0.000)$, previous operations $(\mathrm{p}=0.013)$, history of seizures $(\mathrm{p}=0.046)$, diagnosis of myelomeningocele $(\mathrm{p}=0.046)$, and increased lumbar lordosis at follow-up $(\mathrm{p}=0.015)$ were identified as characteristic risk factors for perioperative infection.

Conclusion: Our results suggest that within the population diagnosed with neuromuscular scoliosis, SCI patients with preexisting pulmonary compromise and greater intraoperative blood loss have the greatest risk of experiencing a major perioperative complication following surgical deformity correction.

422 Differences in Presentation and Revision Rates of Unilateral and Bilateral Rod Fractures in Long, Thoracolumbar Fusion for Adult Spinal Deformity

Munish C. Gupta, MD; Thamrong Lertudomphonwanit, MD; Keith Bridwell, MD; Alekos Theologis; Prachya Punyarat, MD; Brenda A. Sides, MA

Introduction: Rod fracture (RF) is a frequent occurrence after adult spinal deformity (ASD) surgery. Little is known about the association between characteristics of RFs and clinical outcomes. The objective of this study was to compare differences in patients who sustained unilateral(URF) or bilateral (BRF) rod fractures.

Methods: Retrospective review of adults who underwent posterior spinal instrumented fusions (including sacrum; $=5 \mathrm{lev}$ els) from 2004 to 2014 at a single center. Patients were divided into 2 groups based on the number of RFs: URF v. BRF. Each group was subdivided based on the degree of displacement of RF. Demographics, operative data, radiographic parameters, clinical outcomes and revision rates were compared between the groups at baseline and at follow-up.

Results: Of 526 patients who met inclusion criteria, 96 (18.3\%) developed RF [URF: $\mathrm{n}=70$ (73\%); BRF: $\mathrm{n}=26(27 \%)$ ]. Pre-operative demographics and surgical parameters were similar between the groups. At the time of RF occurrence, BRFs had greater SVA $(7.8 \mathrm{~cm}$ v. $4.3 \mathrm{~cm}, \mathrm{p}=0.01)$, less lumbar lordosis $\left(-34.5^{\circ}\right.$ v. $\left.-46.9^{\circ}, \mathrm{p}=0.001\right)$, and greater PI-LL mismatch $\left(22.5^{\circ} \mathrm{v}\right.$. $11.6^{\circ}, \mathrm{p}=0.02$ ) compared to URF. The BRF group had a greater percentage of patients who had major symptoms attributable to the RFs $(84.6 \%$ v. $18.6 \%, \mathrm{p}<0.0001)$ and required revision surgery $(84.6 \%$ v. $18.6 \%, \mathrm{p}<0.0001)$. Of those who required revision surgery, $5 \%$ had nondisplaced URF and $87 \%$ had BRF $(\mathrm{p}<0.0001)$. The BRF group had significantly less improvement in ODI scores (6.7 v. 15.3, p=0.02) and SRS-30 scores (0.3 v. 0.7, p=0.003).

Conclusion: Rod fractures are common after ASD operations. Asymptomatic, nondisplaced unilateral RFs appeared to be benign, as they did not commonly jeopardize clinical outcomes or radiographic alignment parameters. Bilateral RFs were associated with more severe sagittal spinopelvic malalignment, worse improvement in functional outcomes, and more often required revision operations than unilateral RFs.
423 The Correlation Between Cervical Alignment and Posterior Cervical Muscle Fatty Infiltration at Baseline in Cervical Deformity Patients

Peter G Passias, MD; Charles Wang; Gregory W. Poorman; Samantha R. Horn; Frank Segreto; Cole Bortz, BA; Han Jo Kim, MD; Virginie Lafage, $\mathrm{PhD}$; Michael Gerling

Introduction: Degenerative changes in the cervical spine often develop insidiously and spinal cord impairment, malalignment, and muscle impairment frequently co-occur. Cervical extensor musculature is known to play an important role in the physical support of the neck, buts its role in the disease history of degenerative cervical disease and surgical correction of degenerative changes is poorly researched in the literature. Muscle fatty infiltration may have an effect on cervical deformity, including success of surgical intervention.

Methods: Consecutive patients undergoing multi-level posterior fusions for cervical deformity were prospectively enrolled by two surgeons. Preoperative cervical extensor musculature (multifidus, semispinalis cervicis, semispinalis capitis, and splenius capitis) were measured from the T2-weighted axial MR images of the intervertebral disc levels from $\mathrm{C} 2-\mathrm{C} 3$ to $\mathrm{C} 6-\mathrm{C} 7$. These measurements included cross-sectional-area, functional-area (fat free area; FFA), and a ratio of FFA to total-area as an indication of fatty-infiltration. T-tests and Pearson-correlation tests were examied how mal-alignment (cervical-lordosis/kyphosis [C2-C7], cervical-Sagittal-Vertical-Angle [C2-C7 cSVA], and CervicalLordosis minus T1-Slope [TS-CL]) correlated to fatty-infiltration and total-muscle-area. Baseline predictors of fatty-infiltration and total-area were determined.

Results: 20 patients were included (Average age: 55.8 years, $70 \%$ female). Fatty-infiltration increased in the inferior aspect of the cervical spine (C2-C3: 0.51 to C6-C7: 0.64, p<0.001). Gender, age, and BMI did not show any significant relationship with fatty-infiltration or total-muscle-area. However, total-muscle-area was significantly lower in smokers (smoker: $532.3 \mathrm{~cm} 2$ vs. nonsmokers: $954.0 \mathrm{~cm} 2, \mathrm{p}=0.031$ ). While fatty-infiltration significantly correlated with cervical-kyphosis $(\mathrm{r} 2=0.529, \mathrm{p}=0.016)$ and TSCL ( $\mathrm{r} 2=0.659, \mathrm{p}-0.028)$, total-muscle-area had no significant correlation with radiographic-sagittal-alignment (C2-C7, cSVA, TS-CL all $p>0.05)$. Fatty-infiltration and total-muscle-area did not correlate with mJOA, NDI, NSR-back-pain, or NSR-neck-pain scores $(\mathrm{p}>0.05)$.

Conclusion: A significant relationship between cervicalsagittal-alignment, positive sagittal-balance, and posterior muscle fatty-infiltration was observed in preoperative cervical deformity patients. Patient factors including gender, age and BMI did not correlate with muscle-area or fatty-infiltration, while smoking negatively correlated with muscle-area.

424 Risk Factors for Wound Infections After Deformity Correction Surgery in Neuromuscular Scoliosis

Muhammad Burhan Ud Din Janjua, MD; Brandon J Toll, BA; Shashank V Gandhi, MD; Joshua M. Pahys, MD; Amer F. Samdani, MD; Steven Wei-Hung Hwang, MD

Introduction: Complications are more prevalent in neuromuscular scoliosis (NMS) surgery. The main objective of this study is to elucidate risk factors in NMS associated with surgical site infection after scoliosis correction surgery.

Methods: A retrospective review was performed of all patients surgically treated for NMS from January of 2008 to December 2016 with a minimum follow-up of 6-month follow-up. Demographics, pre-op to last follow-up radiographic parameters, 
and details regarding infection were reviewed.

Results: Out of 126 patients, 53 males and 49 females with a mean age at surgery was 14.0 years $(2.7 \pm \mathrm{SD})$, follow-up was 2.53 months $(\mathrm{SD} \pm 1.66)$, and mean time to presentation was 2.14 months $(\mathrm{SD} \pm 4.95$. The most prevalent diagnoses were $\mathrm{CP}$ $(26.5 \%)$, SCI $(24.5 \%)$, and neurofibromatosis $(10.8 \%)$. The overall documented perioperative complication rate was $26.5 \%$ (37 complications in 27 patients) with $32.7 \%$ of patients having wound infections; $8.3 \%$ were superficial and $91.7 \%$ deep wound infections. Primary offending pathogens were E. Coli $(41.7 \%)$ and Proteus Mirabilis (25.0\%). Moreover, pulmonary comorbidities $(\mathrm{p}=.007)$, increase in weight $(\mathrm{p}=.010)$, and lumbar lordosis at follow-up $(\mathrm{p}=.008)$ were significant predictors. Additionally, history of seizures $(\mathrm{p}=.046)$, myelomeningocele repair $(\mathrm{p}=.046)$, previous operations $(\mathrm{p}=.013)$, change in thoracic kyphosis $(\mathrm{p}=.026)$, and shoulder imbalance $(\mathrm{p}=.028)$ were also associated risk factors identified. $6.3 \%$ of wound infections (superficial) were treated with IV antibiotics alone, $93.8 \%$ were treated with wound exploration (deep) and IV antibiotics. Change from preop to immediate post-op proximal and thoracic kyphosis, lumbar lordosis, shoulder imbalance, and pelvic obliquity were not a predictor of wound infection through linear regression analysis.

Conclusion: Wound infection is a major complication after scoliosis surgery in neuromuscular patients. Increase in body weight, lumbar lordosis, pulmonary comorbidity, myelomeningocle repair, seizures, change in thoracic kyphosis, and previous operations were significant predictor identified. Our data highlights major risk factors in this subset of patient population.

425 The Predictable Factors of the Postoperative Kyphotic Change of Sagittal Alignment of the Cervical Spine After the Laminoplasty

Dong Wuk Son; Kyoung Hyup Nam; Geun Sung Song; Sang Weon Lee

Introduction: Laminoplasty is an effective surgical method for treating cervical degenerative disease. However, postoperative kyphosis were often reported. Despite sufficient preoperative lordosis, this procedure often aggravates the lordotic curve of the cervical alignment. Hence, it is important to examine preoperative risk factors associated with postoperative kyphotic changes. Our study aimed to investigate preoperative radiologic parameters associated with postoperative kyphotic deformity.

Methods: We retrospectively reviewed the medical records of 49 patients who underwent open door laminoplasty for cervical spondylotic myelopathy (CSM) or OPLL between January 2011 and December 2015. The radiologic parameters examined included C2 C7 Cobb angles, T1 slope, C2 C7 sagittal vertical axis (SVA), range of motion (ROM) from $\mathrm{C} 2 \mathrm{C} 7$, segmental instability, and T2 signal change observed on magnetic resonance imaging (MRI). Clinical factors examined included preoperative modified Japanese Orthopedic Association scores,disease classification, duration of symptoms, and the range of operation levels.

Results: Mean preoperative sagittal alignment was $13.01^{\circ}$ lordotic; $6.94^{\circ}$ lordotic postoperatively. Percentage of postoperative kyphosis was $80 \%$. Patients were subdivided into two groups according to postoperative Cobb angle change; a control group $(n=22)$ and kyphotic group $(n=27)$. The kyphotic group consisted of patients with more than $5^{\circ}$ kyphotic angle change postoperatively. There were no differences in age, sex, C2 C7 Cobb angle, T1 slope, C2-C7 SVA, ROM, segmental instability, or T2 signal change. Multiple regression analysis revealed T1 slope had a strong relationship with postoperative cervical kyphosis. Likewise, correlation analysis revealed there was a statistical significance between $\mathrm{T} 1$ slope and postoperative Cobb angle change $(\mathrm{p}=0.035)$, and that there was a statistically significant relationship between T1 slope and C2 C7 SVA ( $p=0.001)$. Patients with higher preoperative $\mathrm{T} 1$ slope demonstrated loss of lordotic curvature postoperatively.

Conclusion: Laminoplasty has a high probability of aggravating sagittal balance of the cervical spine. T1 slope is a good predictor of postoperative kyphotic changes of the cervical spine.

426 Rod Fracture in Adult Spinal Deformity Surgery Fused to the Sacrum: Prevalence, Risk Factors and Impact on Health Related Quality of Life in 526 Patients

Munish C. Gupta, MD; Thamrong Lertudomphonwanit, MD; Michael P Kelly, MD; Keith Bridwell, MD; Lawrence Lenke, MD; Steven McAnany; Prachya Punyarat, MD; Jacob Buchowski, MD; Tim Bryan, MD; Lukas Zebala, MD; Brenda A Sides, MA; Karen Steger-May, MA

Introduction: Limited data analysis exist on rod fracture (RF) following posterior spinal fusion to the sacrum to treat adult spinal deformity (ASD). Our study evaluated the prevalence of and risk factors for RF and determined outcomes changes associated with RF after ASD surgery.

Methods: We performed a retrospective single-center analysis of ASD patients (age $>18$ years) undergoing $=5$ vertebrae posterior fusion to the sacrum from 2004 to 2014 . Patients were included if they demonstrated RF occurrence or did not develop RF with a minimum 2-year follow-up. We analyzed baseline demographic, radiographic, clinical outcomes, and operative data. We identified risk factors for RF using separate Cox proportional hazard models based on rod material and diameter.

Results: Five hundred twenty-six patients $(80 \%)$ were included. RF occurred in 97 patients $(18.4 \%)$. Risk factors for fracture of $5.5 \mathrm{~mm} \mathrm{CoCr}$ instrumentation (CC 5.5 model) included preoperative sagittal vertical axis (hazard ratio (HR), 1.07 (95\% confidence interval $[95 \% \mathrm{CI}], 1.02$ to 1.14$)$ per $1-\mathrm{cm}$ increase), preoperative thoracolumbar kyphosis (HR, 1.02 [95\%CI, 1.01 to 1.04] per 1-degree increase), and number of levels fused for patients that received rhBMP-2 $<12 \mathrm{mg}$ per level fused (HR, 1.48 [95\%CI, 1.20 to 1.82 ] per 1-level increase). $5.5 \mathrm{~mm} \mathrm{CoCr}$ implants were at a higher risk for fracture than $6.35 \mathrm{~mm}$ stainless steel (SS) constructs (HR, 8.49 [95\%CI, 4.26 to 16.89]). The RF group had less overall improvement in SRS Satisfaction (0.93 vs. 1.32, $\mathrm{p}=0.007)$ and SRS Self-image domain scores $(0.72$ vs. $1.02, \mathrm{p}=0.01)$.

Conclusion: The prevalence of RF after index procedure was $18.4 \%$. Greater preoperative sagittal vertical axis, greater preoperative thoracolumbar kyphosis, increased number of vertebrae fused for patients received rhBMP- $2<12 \mathrm{mg}$ per level fused, and $\mathrm{CoCr} 5.5 \mathrm{~mm}$ rod were associated with RF risk. Less improvement in patient-satisfaction and self-image was noted in the RF group.

427 Treatment of the Fractional Curve Only in Adult Scoliosis: Comparison to Lower Thoracic and Upper Thoracic Fusions

Dominic Amara, BA; Sigurd Berven, MD; Christopher P. Ames, MD; Bobby Tay, MD; Vedat Deviren, MD; Shane Burch; Praveen V. Mummaneni, MD; Dean Chou, MD

Introduction: Radiculopathy from the fractional curve, typically L4-S1, is frequently a reason for scoliosis patients to pursue surgical intervention. The purpose of this paper is to evaluate treatment outcomes of limited fusion of the fractional curve (FC) only compared to treatment of the entire deformity with long fusion.

Methods: 99 consecutive adult scoliosis patients from 20122016 were retrospectively studied at our institution. Patients with 
fractional curves $>10^{\circ}$ underwent 3 categories of surgeries: 1) fractional curve only ( $\mathrm{FC}, \mathrm{n}=27), 2$ ) lower thoracic to sacrum (LT, $n=46$ ), or 3) upper thoracic to sacrum (UT, n=26). Primary outcomes were the rates of surgical revision surgery and complications. Secondary outcomes were estimated blood loss, length of hospital stay, and discharge destination.

Results: There were no significant preoperative differences in age, gender, smoking status, prior operation, fractional curve degree, pelvic tilt (PT), sagittal vertical axis (SVA), coronal balance, pelvic incidence/lumbar lordosis mismatch (PI-LL), or the proportion of balanced spines $\left(\mathrm{SVA}<5 \mathrm{~cm}, \mathrm{PI}-\mathrm{LL}<10^{\circ}\right.$ and $\mathrm{PT}<20^{\circ}$ ) between the three treatment groups. Mean follow-up was 30.3 (range 12-101) months. The FC group had a lower complication rate $(22 \%[\mathrm{FC}]$ vs $57 \%[\mathrm{LT}]$ vs $58 \%$ [UT], $\mathrm{p}=0.009)$, but a higher rate of extension surgery $(26 \%$ [FC] vs $13 \%$ [LT] vs $4 \%$ [UT], $\mathrm{p}=0.068$ ). The respective (FC, LT, UT) average estimated blood loss (593cc vs $1950 \mathrm{cc}$ vs $2634 \mathrm{cc}, \mathrm{p}<0.001)$, length of hospital stay (5.7 vs 8.3 vs 8.3 days, $\mathrm{p}=0.002$ ) and rate of discharge to acute rehabilitation ( $30 \%$ vs $45 \%$ vs $85 \%, \mathrm{p}<0.001)$ were all lower for FC and highest for UT.

Conclusion: Treatment of the FC only is associated with a lower complication rate, shorter hospital stay and lower blood loss than complete scoliosis treatment. However, there is a higher associated rate of extension of the construct to the LT or UT, and patients should be counseled when considering their options.

428 Triangle Offense: A Three-surgeon Approach to Reduce Complications in Cervical and Cervical-thoracic Deformity Surgery, A Prospective Cohort Study

Vijay Yanamadala, MD, MBA; Bryan D. Choi, MD; Ganesh Shankar, MD, PhD; Laura Van Beaver; Thomas D. Cha, MD; Kyle Eberlin, MD; John H. Shin, MD

Introduction: Complication rates after adult spinal deformity surgery range as high as $80 \%$. Multidisciplinary approaches can reduce complications associated with these surgeries. Herein, we present our multidisciplinary team-based operative approach for cervicothoracic deformity.

Methods: An IRB approved analysis was performed from a prospective database from 2011-2017. Patients undergoing any combination of anterior and/or posterior osteotomy surgeries for cervical and cervicothoracic deformities were evaluated pre-operatively by a neurosurgeon, orthopaedic spine surgeon, and plastic surgeon who developed an operative plan together including plans for deformity correction and wound closure. Pre-operative imaging including MRI, CT, AP, lateral, flexion/extension cervical, and standing scoliosis $\mathrm{x}$-rays were performed in all patients. Spinal exposure, instrumentation, and osteotomies were performed by the neurosurgeon and orthopaedic surgeon using a two-attending model. Wound closure with flap reconstruction when necessary was performed by the plastic surgeon. Post-operatively patients were transferred to an intensive care unit for overnight monitoring. Patients mobilized with physical therapy on post-operative day one. A wound-care specialized nurse managed daily dressing changes starting post-operative day two. Strict calorie counts were conducted by staff nutritionists to ensure caloric intake meeting a minimum of $60 \%$ of needs starting post-operative day two.

Results: 54 consecutive patients underwent surgery for cervical deformity, including patients with symptomatic cervical and cervicothoracic kyphosis greater than 25 degrees or cervical scoliosis greater than 30 degrees. Sixteen patients underwent pedicle subtraction osteotomy. Eleven patients underwent vertebral column resection. Nineteen patients underwent combined anterior/ posterior approaches including multi-level osteotomies. All patients underwent posterior instrumentation involving at minimum C2 T2 (range: occiput T10). Twenty-four patients required local myocutaneous flap extension during closure. Eleven patients required transfer myocutaneous flap reconstruction. The overall complication rate, including death, myocardial infarction, thrombotic events including pulmonary embolism and deep venous thrombosis, systemic infection including pneumonia and urinary tract infection, was $6 \%$. There were no post-operative wound infections or wound dehiscence at last follow-up (median follow-up 34 months; range: 3 months 5 years). Mean hospital length of stay was 6.7 days (range 413 days).

Conclusion: Multidisciplinary surgical decision making can substantially reduce complications associated with cervical deformity surgery, maximizing the efficacy and safety of these complex surgeries.

429 Perioperative Transfusion Threshold of Hemoglobin e" 9.0mg/dL Results in Better Early Ambulation and Shorter Length of Stay for Patients Undergoing Adult Spinal Deformity Surgery

Vijay Yanamadala, MD, MBA; Anna Wright; Jean-Christophe A. Leveque, MD; Rajiv Sethi, MD

Introduction: While a transfusion threshold of hemoglobin $=7.0 \mathrm{mg} / \mathrm{dL}$ has been established as adequate for most adult patients, patients with acute blood loss or those with acute coronary syndromes have demonstrated better outcomes with a more liberal transfusion threshold hemoglobin $=10 \mathrm{mg} / \mathrm{dL}$. No transfusion threshold has been set for patients undergoing complex spine surgery.

Methods: Retrospective review of one hundred consecutive patients who underwent surgery for the correction of adult spinal deformity with greater than six levels of posterolateral thoracolumbar fusion with interbody fusion was carried out. Patient demographics, details of the surgical procedure, post-operative day 1 and day 2 hemoglobin levels, day of first ambulation, length of stay and 30-day and 90-day complications were obtained and assessed by multivariate logistic regression.

Results: Mean length of stay was 12.1 days, including a mean 2.1 days between procedures where staged. Mean post-operative day 1 hemoglobin was $9.9 \mathrm{mg} / \mathrm{dL}$ (range 8.3 to 11.9 ). Patients with post-operative day 1 hemoglobin $=9.0 \mathrm{mg} / \mathrm{dL}$ had a shorter length of stay after final surgical stage vs. those who had a hemoglobin < $9.0 \mathrm{mg} / \mathrm{dL}$ (10.5 days vs. 14.1 days; $\mathrm{p}<0.05)$. Patients with a higher hemoglobin also demonstrated earlier first ambulation (1.6 days vs. 2.7 days; $p<0.05)$. There were no significant demographic differences between these two groups. A hemoglobin threshold of $10.0 \mathrm{mg} / \mathrm{dL}$ did not confer any advantage with respect to length of stay or ambulation. There was no difference in complication rates or 30-day readmission rates between the two groups.

Conclusion: Complex spine surgery for the correction of adult spinal deformity involves massive blood loss and persistent blood loss over the first 48 hours of surgery. Aggressive transfusion with a peri-operative transfusion threshold hemoglobin $=9.0 \mathrm{mg} / \mathrm{dL}$ appears to allow these patients to ambulate earlier and have shorter hospital stays without additional complications.

430 Do Lumbar Lordosis (LL) and Pelvic Incidence (PI) Correlate Among Subjects 4-15 Years of Age and Can LL Predict PI

Lawrence Lenke, MD; Saumyajit Basu, MD; Fernando Emilio Silva, MD; Dharmesh Patel, MD; Amitava Biswas, MD; Yang Yang

Introduction: Deformity surgical practice involves using pelvic incidence (PI) to establish postop lumbar lordosis (LL).The 
latter has been established in adults.It is unclear in the pediatric population whether or not PI can be used to establish postopLL.

Methods: One-hundred and thirty subjects ages 4-15 years were studied.Among several sagittal and spinopelvic paramenters measured, PI and LL were studied.Using correlation analysis, the association between LL and PI was studied in the entire sample population, then among ages 4-10 and 11-15.Using regression analysis, the prediction of LL from PI for preoperative planning was studied among the same population and same age groups, as in our correlation analysis.

Results: To study the association between LL and PI, we ran correlation studies.In 4-15 group, the correlation was 0.38937 with $\mathrm{p}$ value of $<0.0001$. In the 4-10 and 11-15 groups, correlations were 0.13894 with $p$ value of 0.2345 and 0.54847 with $p$ value of $<0.0001$, respectively.We ran regression analysis to predict LL from PI.In the 4-15 group, linear regression with a simple linear term yielded $\mathrm{R} 2=0.15$ and linear regression with a spline function $\mathrm{R} 2=0.20$.Regression data was studied with groups 4-10 and 11-15. For 4-10 years,linear regression with a simple linear term yielded $\mathrm{R} 2=0.08$ and linear regression with a spline function $\mathrm{R} 2=0.14$. For 11-15 group linear regression with a simple linear term yielded $\mathrm{R} 2=0.24$ and linear regression with a spline function $\mathrm{R} 2=0.28$.

Conclusion: LL/PI association in the 4-15 group was weak. This association remained weak in the 4-10 group; stronger among the 11-15 group.Regression analysis in the 4-15 group was also weak.Using single variable PI to predict LL, we conclude that overall the prediction is not very accurate with simple linear term, and improve marginally with a restricted cubic spline function. Prediction is better in the 11-15 than 4-10 group, although the predictive accuracy not promising in both.

431 A limited Surgery May Adequately Address Pain and Improve Quality of Life in Patients with Associated Lumbar Scoliosis

Joseph Molenda, MD; Mena G Kerolus, MD; Ryan Michael Glynn; Richard G. Fessler, MD, PhD

Introduction: Options for surgical treatment of adult degenerative scoliosis vary from decompression, decompression with a short focal fusion, and decompression with long fusion achieving full correction of scoliosis. Decompression alone risks instability, progression of deformity, as well as symptom recurrence. Long fusions in elderly patients also may result in higher rates of complications, longer OR times, increased blood loss, and a prolonged hospital stay. Therefore, focal decompression with fusion often represents an appropriate option. Our study intends to look at patients with lumbar scoliosis undergoing MIS TLIF for neurogenic symptoms, without specific correction of the scoliosis.

Methods: This study retrospectively evaluated collected spine registry data at a single institution performed by a single surgeon. Inclusion criteria were 1) radicular symptoms or neurogenic claudication with or without back pain 2) symptoms refractory to conservative therapy 3) correlated positive radiological findings 4) one level MISTLIF procedure performed 5) minimum 1 year follow-up; and 6) coronal cobb angle $>10$ degrees. Exclusion criteria were those with previous spinal instrumentation or acute trauma. Clinical outcomes assessed included ODI, NRS, SRS 30, VR12 and VR6D, and SF12. All scores were further subdivided by Cobb angle (10-19, 20-29, and > 30).

Results: 24 total patients underwent a one level MISTLIF with pain assessment scores. 16 completed preop scores, 22 at 3 months, 14 at 6 months, 15 at one year, and 9 at 2 years. NRS back scores trended with leg scores. Preop NRS back scores averaged 6.25 and decreased to 2.7 at 3 months, 3 at 6 months, and 3.1 at 1 year. ODI scores also improved over this time period.
Conclusion: This study demonstrates that for a specific subset of patients undergoing one level MISTLIF, minimal operations are benfeficial. Further work evaluates patients undergoing one level ALIF, microendoscopic decompression of stenosis, and microforaminotomies.

432 The Comprehensive Anatomical Spinal Osteotomy and Anterior Column Realignment Classification

David S. Xu, MD; Jacob Januszewski, DO; Frank Schwab, $\mathrm{MD}$, PhD; Juan S. Uribe, MD, FAANS

Introduction: Spinal osteotomies and anterior column realignment (ACR) are procedures that allow for preservation or restoration of spine lordosis. Variations of this technique enable different degrees of segmental, regional, and global sagittal realignment. We propose a comprehensive anatomical classification system for ACR and its variants based on the level of technical complexity and invasiveness. This serves as a common language and platform to standardize clinical and radiographic outcomes for utilization of ACR.

Methods: he proposed classification is based on 6 anatomical grades of anterior column realignment including anterior longitudinal ligament (ALL) release, with varying degrees of posterior column release/osteotomies. Additionally, a surgical approach (anterior, lateral, posterior) and multiplicity (single- or multi-level) modifier is added. Reliability of the classification was evaluated by an analysis of 16 clinical cases, rated 2 times by 14 different spine surgeons and calculation of Fleiss kappa coefficients.

Results: The 6 Grades are as follows: Grade 0, ALL release with hyperlordotic cage, intact posterior elements. Grade I $(A C R+1)$, additional resection of the inferior facet and joint capsule. Grade $2(\mathrm{ACR}+2)$, additional resection of both superior and inferior facets, interspinous ligament, ligamentum flavum, lamina and spinous process. Grade $3(\mathrm{ACR}+3)$ : additional adjacent level 3 column osteotomy including pedicle subtraction osteotomy. Grade $4(\mathrm{ACR}+4)$ : additional adjacent partial vertebral body and disc resection. Grade $5(\mathrm{ACR}+5)$ : complete removal of a vertebral body and both adjacent discs with or without posterior element resection. Intraobserver and interobserver reliability was classified $97 \%$ and $98 \%$ respectively.

Conclusion: The proposed anatomical realignment classification provides a consistent description of the various posterior and anterior column release/osteotomies. The reliability study confirmed that the classification is consistent and reproducible.

433 Does Prior Spine Surgery or Instrumentation Affect Surgical Outcomes Following Three-column Osteotomy for Correction of Thoracolumbar Deformities?

Darryl Lau, MD; Andrew Kai-Hong Chan, MD; Vedat Deviren, MD; Christopher P. Ames, MD

Introduction: Adult spinal deformity is most commonly due to asymmetric arthritic degeneration and/or iatrogenic causes such as prior spinal instrumentation. Many patients who present with spinal deformity have undergone prior spine surgery. There is lack of contemporary studies that evaluate whether prior surgery and/or instrumentation affects perioperative outcomes, readmission, and need for reoperation.

Methods: All adult patients who underwent three-column osteotomy for correction of thoracolumbar spinal deformity from 2006-2016 were identified. We compared outcomes between primary (first-time) vs. revision cases, concentrating on number of prior surgeries $(0,1,2,3,4$, and 5 or more) and prior instrumentation. Multivariate analysis was used to adjust for relevant and significant confounders. 
Results: A total of 300 cases were included, and $38.3 \%$ were male. Overall complication rate was $24.7 \%$ and mean hospital stay was 8.1 days. Ninety-day readmission rate was $9.0 \%$ and reoperation rate was $26.7 \%$. There was no significant difference in complication rate $(26.6 \%$ vs. $24.0 \%, \mathrm{p}=0.645)$, hospital stay ( 8.7 vs. 7.9 days, $\mathrm{p}=0.229)$, readmission rate $(11.4 \%$ vs. $8.1 \%, \mathrm{p}=0.387)$, and reoperation rate $(26.6 \%$ vs. $26.7 \%, \mathrm{p}=0.984)$ between primary vs. revision cases. There was no significant difference in wound infections and dehiscence requiring reoperation $(5.1 \%$ vs. $6.3 \%$, $\mathrm{p}=0.683$ ). When analyzed based on the number of prior spine surgeries or history of spinal instrumentation, no significant differences were observed for all outcomes of interest. Additionally, after adjusting for covariates on multivariate analysis, there were no significant associations between prior surgical histories (primary vs. revision, number of prior surgeries, and prior instrumentation), and all of the outcomes of interest.

Conclusion: The findings from this study suggest that patients who have undergone prior spine surgery with or without instrumentation are not at increased risk for perioperative complications, readmission, or reoperations following three-column osteotomy. These findings were also seen for patients that had undergone 5 or more prior surgeries.

434 The Utility of Vertebral Column Subtraction Osteotomies For Acquired and Congenital Tethered Cord Syndrome

\section{Shashank V Gandhi, MD; Ahmad Latefi}

Introduction: Detethering of the spinal cord has historically been the treatment of choice for TCS in the setting of spinal dysraphisms. Recurrent TCS occurs 5-80\% of patients, with $27 \%$ risk of neurological injury, CSF leak, and wound breakdown that increase with subsequent procedures. Vertebral column shortening osteotomy (VCSO) without microsurgical detethering to relieve stretch strain on the spinal cord has been described for congenital TCS; however, there is a paucity of literature on its success. Here we describe our experience with VCSO for acquired and congenital TCS.

Methods: A prospectively collected database with TCS patients who underwent VCSO was analyzed. Preoperative evaluation included MRI and upright radiographs to assess for location and type of tethering and presence of deformity. Single stage posterior VCSO were conducted at a neutral level rostral to the tethering site, shortening by $10-15 \mathrm{~mm}$. When present VCSOs were concurrently utilized to correct deformities.

Results: 5 patients underwent VCSO. 3 patients had failed previous microsurgical detethering at least once. Etiology of TCS were: 2 with history of myelomeningocele, 2 with arachnoid adhesions after spinal subdural hematoma and 1 with arachnoiditis and footdrop after a lumbar microdiscectomy complicated by a durotomy. Radicular pain improved in all patients and resolved in 3 . Strength improved by atleast 1 grade. The patient with foot-drop regained 4/5 strength. Sensation improved in all patients except that with foot-drop. Only $1 / 3$ patients with bladder incontinence improve. The 2 that did not had baseline incontinence from myelomeningocele. There was no worsening of neurological function or recurrence of symptoms. 4/5 patients improved in ambulation.

Conclusion: VCSO can be safely used for acquired and congenital TCS with improvement in motor, sensory, and pain symptoms. Indirect relief of stretch on can be achieved without recurrence symptoms or neurological injury as there is no direct manipulation of neural elements.
435 Perioperative Outcomes Associated With Thoracolumbar Three-Column Osteotomies for Adult Spinal Deformity Patients With Rheumatoid Arthritis

Cecilia L. Dalle Ore, B.A.; Darryl Lau, MD; Vedat Deviren, MD; Christopher P. Ames, MD

Introduction: Spinal deformity causing spinal imbalance is a significant cause of pain and disability. Prior studies suggest that patients with rheumatoid arthritis (RA) undergoing thoracolumbar deformity correction are at an increased risk for complications. This study compares outcomes of RA patients following threecolumn osteotomies to a matched control cohort.

Methods: All patients with RA who underwent three-column osteotomies for thoracolumbar deformity correction by the senior author from 2006 to 2016 were identified retrospectively. A matched cohort of patients without RA was generated. Data regarding demographics, surgical approach, and endpoints including complications, reoperations, and incidence of proximal junctional kyphosis (PJK) were reviewed and compared.

Results: Eighteen patients with RA were identified, and a matched cohort of 228 patients was generated. With regards to patients with RA, $11.1 \%$ were male and the mean age was 68.1 years. Vertebral column resection (VCR) was performed in $22.2 \%$ and pedicle subtraction osteotomy (PSO) in $77.8 \%$. Mean case length was 324.4 minutes and estimated blood loss (EBL) was $2053.6 \mathrm{ml}$. There was no significant difference in case length or EBL between RA patients and matched controls. Complications occurred in $26.4 \%$ of patients overall: $38.8 \%$ of patients with RA and $20.1 \%$ of patients without RA ( $p=0.213$ ). Patients with RA had a significantly higher incidence of DVTs $(11.1 \%$ versus $1.7 \%$, $\mathrm{p}=0.013$ ). PJK occurred in $16.7 \%$ of patients with RA and $33 \%$ of patients were reoperated. Incidence of PJK and reoperation in matched controls were $8.8 \%$ and $25 \%$, respectively $(\mathrm{p}=0.361, \mathrm{p}$ $=0.435$ ).

Conclusion: Findings from this study suggests that RA have higher incidence of medical complications, specifically deep vein thrombosis (DVT). Early initiation of DVT prophylaxis in RA patients may be indicated. Perioperative complications, morbidity, and long-term outcomes are otherwise similar to a matched cohort of non-RA patients.

436 Changes in Pelvic Incidence After Adult Deformity Surgery

\section{Christian B Ricks, MD; Adam S. Kanter, MD}

Introduction: The authors challenge the dogma that pelvic incidence is a fixed parameter by investigating changes after spinal deformity surgery.

Methods: Measurements of the preoperative and postoperative pelvic incidence (PI), pelvic tilt (PT), sacral slope (SS), and lumbar lordosis (LL) were performed using Surgimap software and confirmed with conventional manual measurements. Preoperative and postoperative outcomes, including Oswestry Disability Index (ODI) were prospectively gathered. A paired t-test was used to compare changes in pelvic incidence.

Results: 29 patients, averaging 60.4 years, preoperative ODI 49.4, VAS 7.3, PI 60.0, PT 28.9, and PI-LL mismatch of 25.4 underwent surgical correction fusing an average of 10.6 spinal levels. 14 were fused to the pelvis, of which 3 were iliac screws and 11 were S2-alar screw fixation. 6 month postoperative averages were ODI 41.8, VAS 4.4, PI 62.4, PT 30.2, and PI-LL mismatch of 17.3. 10 patients required extension of fusion operations within 5 years. Changes in PI compared to preoperative values were statistically significant for those undergoing pelvic fixation $(n=14)$ at 
6 months $(\mathrm{p}=.04)$ and 24 months $(\mathrm{p}=.05)$, representing an average of 2 degrees. There were no changes in PI after surgery comparing 6 months and 24 months $(\mathrm{p}=.71)$. Those without pelvic fixation $(n=15)$ did not have statistically significant changes in PI. Among those fused to pelvis, iliac screws $(n=3)$ did not show significant changes in PI, while those with S2-alar screws did at 6 months $(\mathrm{p}=.01)$ and 24 months $(\mathrm{p}=.02)$. Those who required a reoperation within 5 years had a statistically significant change in PI $(p=.04)$. Analyzing the correlation between postoperative change in PI among S2-alar fused patients showed an $\mathrm{R}=-.44$ for ODI and $\mathrm{R}=$ -.63 for PI-LL mismatch.

Conclusion: Pelvic incidence changes in patients undergoing sagittal deformity correction with S2-alar screws. This may represent shear forces across the sacro-iliac joint which change from a standing to prone position, and become immobilized with $\mathrm{S} 2$-alar fixation. This is supported by reasonable correlation with preoperative PI-LL mismatch, and may be used to help predict those who will require eventual revision surgery.

437 The Unreimbursed Costs of Preventing Revision surgery in Adult Spinal Deformity: Analysis of Cost Effectiveness of Pseudoarthrosis and Revision Prevention Strategies in 195 Patients

Michael Safaee, MD; Cecilia L. Dalle Ore, B.A.; Corinna Clio Zygourakis, MD; Vedat Deviren, MD; Christopher P. Ames, MD

Introduction: Surgical adjuncts such as osteoinductive biologics and PJF prevention strategies in adult spinal deformity (ASD) have the potential for significant cost savings by preventing readmission and reoperation, yet are often unreimbursed. Hospitals often bear this additional cost despite potential lasting benefit to the patients.

Methods: Single center, retrospective review of ASD patients.

Results: 195 patients were identified with a mean age of 64 years including 135 women and 60 men. An average of 10 levels were fused (range 2-18) with upper instrumented vertebrae (UIV) as follows: 2 cervical, 73 upper thoracic, 108 lower thoracic, and 12 lumbar. Surgical adjuncts included bone morphogenetic protein (BMP) in 132 cases, ligament augmentation in 99 cases, vertebroplasty in 71 cases, and UIV hook fixation in 60 cases. The average cost of index surgery was $\$ 129,953$. Seventeen patients (9\%) underwent surgical revision for pseudarthrosis with a mean follow-up of 22 months. Fewer levels fused, BMP, vertebroplasty, and ligament augmentation were associated with lower rates of revision surgery. For 3 column osteotomies, use of a 4 rod technique was associated with a significant reduction in revision surgery ( $6 \%$ vs. $29 \%, p=0.016$ ). The average cost was $\$ 9,099$ for BMP, $\$ 1,184$ for ligament augmentation, $\$ 1,176$ for vertebroplasty, and $\$ 2,738$ for the 4 rod construct. The average cost of reoperation was $\$ 95,852$. The average risk reduction per adjunct was $10 \%$, with a number needed to treat of 10 patients.

Conclusion: BMP, ligament augmentation, vertebroplasty, and 4 rod constructs were associated with reductions in pseudarthrosis-related revision surgery. These adjuncts cost an average of $\$ 14,197$ per patient ( $11 \%$ of index surgery) but in a population of 1,000 ASD patients have the potential to prevent 100 revision surgeries for a savings of $\$ 9,585,200$. When used in combination, these strategies have the potential for more dramatic savings by reducing rates of revision surgery.

438 Perioperative Outcomes Associated with Posterior Vertebral Column Resection for Severe Thoracic Kyphosis

Cecilia L. Dalle Ore, B.A.; Darryl Lau, MD; Vedat Deviren, MD; Christopher P. Ames, MD
Introduction: Thoracic kyphosis can result in neurologic deficits, refractory pain, and cardiopulmonary dysfunction when severe. Vertebral column resection (VCR) is a powerful technique that can be employed for large curves and fixed deformities. This study reports the outcomes of posterior VCR for severe kyphotic deformities.

Methods: A retrospective review of all patients who underwent VCR for severe thoracic kyphosis (defined as segmental kyphosis greater than 80 degrees) was performed. Patients with kyphosis secondary to trauma, tumor, or infection were excluded. Perioperative, radiographic, and minimum 2-year outcomes were assessed.

Results: Nineteen patients were included in the analysis. Mean age was 57.1 years and $31.6 \%$ were male. Median preoperative sagittal vertical axis (SVA) was $44 \mathrm{~mm}$ and thoracic kyphosis (TK) was 86.5 degrees. Among 19 patients, 24 VCR were performed and median 16 levels were fused. Median blood loss was $2000 \mathrm{ml}$. Median length of stay was 6 days. Perioperative complication rate was $36.8 \%$. One patient developed anterior spinal syndrome, cardiomyopathy, and respiratory failure and perished during the index admission. Other complications observed were seizures, volvulus, cord compression at the VCR site, pneumothorax, and wound infection. Median postoperative SVA was 39.5 $\mathrm{mm}$ and TK was 57.5 degrees. Incidence of junctional failure was 14.8\%: 1 proximal and 2 distal. All junctional disease required extension of fusion. At median 24.6 months follow up, $57.9 \%$ of patients reported reduction of back pain and $47.3 \%$ were able to reduce their dose of opioid medications. $78.9 \%$ endorsed an improvement in ambulation and posture.

Conclusion: Posterior VCR is an effective technique in the correction of severe thoracic kyphotic deformities. Perioperative morbidity is not low, but the majority of patients fare well at follow-up and report improvement in ambulation and posture. Junctional disease occurs both proximal and distal; surgeons should implement strategies to minimize both proximal and distal junctional disease.

439 The Relationship of Older Age on Perioperative Outcomes Following Thoracolumbar Three-column Osteotomy for Adult Spinal Deformity: Analysis of $\mathbf{3 0 0}$ Consecutive Cases

Darryl Lau, MD; Joseph A Osorio, MD, PhD; Vedat Deviren, MD; Christopher P. Ames, MD

Introduction: There is hesitation to perform three-column osteotomies for thoracolumbar spinal deformities in older patients due to concern for high morbidity. This study assesses whether age is independently associated with perioperative outcomes.

Methods: All thoracolumbar adult spinal deformity patients who underwent three-column osteotomy by the senior author from 2006 to 2016 were identified. Multivariate analyses were used to assess the association of age with outcomes (perioperative complication, intensive care unit stay (ICU), and length of hospital stay (LOS)).

Results: A total of 300 patients were included, and $38.3 \%$ were male. Mean age was 63.7 years: < 50 years $(10.3 \%), 50$ to 64 years $(36.0 \%), 65$ to 79 years $(45.7 \%)$, and 80 years and older (8.0\%). Overall complication rate was $24.7 \%$ : $18.0 \%$ had a medical complication and $7.0 \%$ had a surgical complication. There were no perioperative or 30-day deaths. Age was associated with higher overall complications $(\mathrm{p}=0.002)$ and medical specific complications $(\mathrm{p}<0.001):<50$ years $(9.7 \%$ and $6.5 \%), 50$ to 64 years $(16.7 \%$ and $10.2 \%), 65$ to 79 years $(31.4 \%$ and $22.6 \%)$, and 80 years or older (41.7\% and $41.7 \%$ ). Age was not an independent factor for complications. Surgical complication rates were similar. Longer 
ICU and LOS were observed in older age groups, and age was an independent factor associated with longer ICU $(\mathrm{p}=0.028)$ and LOS $(p=0.003):<50$ years (1.6 days), 50 to 64 years ( 2.3 days), 65 to 79 years (2.0 days), and 80 years or older (3.2 days). LOS stratified by age were: $<50$ years ( 7.3 days), 50 to 64 years ( 7.7 days), 65 to 79 years (8.2 days), and 80 years or older (11.0 days).

Conclusion: Older age was associated with higher complication rate but age alone was not an independent risk factor. Comorbidities and other unknown variables that come with age are likely what put these patients at higher risk.

440 Impact of Perioperative Neurological Deficits on Clinical Outcomes Following Posterior Cervical Fusion

Nitin Agarwal, MD; Sanjana Dayananda, B.S.; Amol Mehta, BA; Enyinna Levi Nwachuku, MD; D. Kojo Hamilton, MD; Parthasarathy D Thirumala

Introduction: In the United States, the number of posterior cervical fusions has increased substantially. Perioperative neurological complications associated with this procedure, such as spinal cord and peripheral nerve injuries, can have dangerous effects on patient health. As such, we examined the impact of perioperative neurological deficits on mortality in patients undergoing posterior cervical fusion. The secondary aim was to understand the risk factors for perioperative neurological complications.

Methods: Data was collected from the National Inpatient Sample (NIS) Health Cost Utilization Project (HCUP) between 1999 and 2011. Patients below the age of 18 and above the age of 80 were excluded, as were patients who underwent posterior cervical fusion due to trauma or malignancy. Patient demographics and comorbidities were compiled as well as variables that have been associated with increased risk of perioperative neurological complications. We utilized the van Walraven score, a weighted numerical surrogate for the Elixhauser comorbidity index, as a covariate to assess comorbidities that have been associated with in-hospital mortality and morbidity after posterior cervical fusion. Additionally, we performed univariate comparisons between covariates and surgical outcomes. We conducted a multivariable logistic regression, adjusting for many of the covariates, as well as trend analyses through a univariate logistic regression.

Results: An analysis of 33,690 patients yielded an overall rate of perioperative neurological deficits, morbidity, and mortality of $1.08 \%, 40.44 \%$, and $1.00 \%$, respectively. Perioperative neurological deficits were indeed independent risk factors predictors of in-hospital mortality (OR 5.270; $\mathrm{p}<0.0001$ ) and morbidity (OR $2.579 ; \mathrm{p}<0.0001)$. Other statistically significant predictors of mortality included increasing van Walraven score, myocardial infarction, postoperative pneumonia, and venous thromboembolism. These were also independent predictors of morbidity along with but not limited to urinary, renal, digestive system complications, hematoma/seroma, post-hemorrhagic anemia, dysphagia, device complications, and intraspinal abscess.

Conclusion: Perioperative neurological deficits are serious complications of PCF and can independently predict in-hospital mortality and morbidity. As this procedure continues to be utilized more and more, attention should be directed towards preventing these complications and intervening earlier in patients who have suffered a neurological deficit. Future efforts should be geared towards screening for at-risk patients with the initiation of surgical prehab.

441 Risk Factors Associated with 90-day Readmissions After Degenerative Lumbar Fusion: An Examination of the Michigan Spine Surgery Improvement Collaborative (MSSIC) Registry
Paul Park, MD; David Nerenz, PhD; Michael Bazydlo, MS; Lonni Schultz, PhD; Hesham Mostafa Zakaria, MD; Mark E. Oppenlander, MD; Victor W. Chang, MD

Introduction: Readmission to the hospital negatively impacts the patient experience and is very costly. To date, most studies have evaluated 30-day readmissions after spine surgery. Evaluation of the 90-day period, however, allows a more comprehensive assessment of factors associated with readmission. The purpose of this study is to assess the reasons and risk factors for 90-day readmissions after lumbar fusion surgery.

Methods: The Michigan Spine Surgery Improvement Collaborative (MSSIC) registry is a prospective, multi-center, and spine-specific database of patients surgically treated for degenerative disease. Data from the MSSIC registry was retrospectively analyzed. Causes for readmission were determined and independent risk factors impacting readmission were found by multivariate logistic regression.

Results: A total of 8533 patients who underwent lumbar fusion were identified. Seven hundred sixty-three $(8.9 \%)$ patients were readmitted within the 90 -day period. The most common specified reasons for readmission were surgical site infection (17\%), pain (16\%), and radicular symptoms (10\%). Risk factors associated with increased likelihood of readmission were diabetes (OR 1.54, CI 1.27-1.85), 4 or more fused levels (OR 1.48, CI 1.141.92), surgery length (OR 1.09, CI 1.04-1.15), and hospital length of stay (OR 1.01, CI 1.00-1.02). Factors associated with decreased risk of readmission were discharge to home (OR 0.71, CI 0.590.86 ), private insurance (OR $0.81, \mathrm{CI} 0.68-0.96)$, spondylolisthesis diagnosis (OR 0.84, CI 0.72-0.98).

Conclusion: After lumbar fusion surgery, 90-day readmission to the hospital happens in approximately $9 \%$ of cases. Among many reasons for readmission, the most frequent reasons included wound infection, pain, and radicular symptoms. Among many factors impacting the likelihood of 90-day readmission, diabetes was the highest risk factor and should be a condition that is strictly managed preoperatively and throughout the operative and postoperative period.

442 The Utilization of Machine-learning Algorithm for Generating Interactive 3-Dimensional Holographic Images from Standard Computer-tomographic and Magnetic Resonance Imaging Modalities: The Future of Intra-operative Navigation Modules

Jang Yoon, MD, MS; Louis J Kang, MS, BS; Robert E Chen; Esther Kim; Tay Won Kim, BS; Robert E. Wharen, MD; Mohamad Bydon, MD; Mark A. Pichelmann, MD; Alfredo Quinones-Hinoja, MD

Introduction: We developed a fully integrated medical imaging system that performs $3 \mathrm{D}$ reconstructions of pre- and intra-operative images, translates these reconstructed images into holograms, projects holograms onto the surgical field via wearable head-up display, and provides feedback to the surgeon (via the head-up display) in real time.

Methods: We built a fully integrated system that performs 3D reconstruction of CT and MRI images from $2 \mathrm{D}$ z-stacks and relays these into holograms that a surgeon sees with a head-up display. We leveraged deep learning to extract surgical regions of interest (e.g., brain tumors, anatomical landmarks) from pre-operative MRI scans, and perform 3-dimensional reconstructions of them into holograms that are projected onto the surgical field (Figure 1). Specifically, a deep convolutional neural network was used for the segmentation process. The architecture of this deep learning algorithm included embedding layer and 4 convolutional layers 
with dropout between each of the first two, and softmax layer to classify each individual pixel in the image. After segmentation and post-image processing, we trained our software to recognize certain signals within images (i.e. contrast enhancing portion of brain tumors) to automate the reconstruction process. The training is done on a large training set of past MRI images from the Mayo Clinic. Next, we implemented the software on a wearable that projects holographic images, and built interaction software to let the surgeon interact with the 3D holographic images.

Results: The feedback loop is a connection between the head-up display, the navigation probe, the navigation registration receiver, and the navigation module. All communications are performed wirelessly through Bluetooth and $\mathrm{WiFi}$ and tested in real-time on a phantom head (Figure 2).

Conclusion: This study lays the groundwork and the next phase will be the expansion to surgeries involving spine, extremities and skull base. This can also improve pre-operative surgical simulation and resident education.

\section{Academic Productivity of Spine Faculty in Neurological and Orthopedic Surgery Residency Programs}

\section{Alexander F. Post, MD; Tanvir Choudhri, MD}

Introduction: We present a unified analysis of academic productivity for spine-focused faculty at U.S. neurological and orthopedic surgery residency programs.

Methods: 278 ACGME-training programs (110 neurosurgical, 168 orthopedic) were queried to identify 945 full-time spinal surgery faculty (designated by fellowship training or case volume $>75 \%$ spine) and to assess academic rank, gender and academic productivity.

Results: Comparison of neurological and orthopedic surgery showed a similar median h-index (NS:OS $=10: 9)$, identical mode h-index (NS:OS:total $=5: 5)$ and similar faculty $\mathrm{h}$-index ranges (NS:OS $=[0-61]:[0-81])$. A statistically significant correlation (Spearman's Rho $=1, \mathrm{p}=0$ ) of mean $\mathrm{h}$-index with academic rank was seen in neurosurgical (mean h-Instr $=2.75 \pm 2.22$, h-Asst $=$ $7.83 \pm 5.55, \mathrm{~h}$-Assoc $=13.05 \pm 7.79, \mathrm{~h}$-Prof $=24.30 \pm 13.14)$ and orthopedic programs (mean h-Instr $=5.00 \pm 3.96$, h-Asst $=7.27$ $\pm 5.45, \mathrm{~h}$-Assoc $=10.70 \pm 7.07, \mathrm{~h}$-Prof $=26.87 \pm 15.54)$. A higher mean h-index was seen in neurosurgical Assistant and Associate professors and orthopedic Instructors and Professors. Breakdown by gender showed a higher mean h-index for men at each rank. Neurological surgery: mean h-Instr $[\mathrm{M}: \mathrm{F}=4.00 \pm 2.83: 1.50$ $\pm 0.71]$, h-Asst [M:F $=7.87 \pm 5.76: 7.13 \pm 3.68]$, h-Assoc [M:F $=13.26 \pm 7.97: 9.71 \pm 4.461]$ and $\mathrm{h}$-Prof $[\mathrm{M}: \mathrm{F}=24.39 \pm 13.14:$ $16.00 \pm 0]$. Orthopedic surgery: mean h-Instr $[\mathrm{M}: \mathrm{F}=5.00 \pm 39.6$ $: \mathrm{n} / \mathrm{a}]$, h-Asst $[\mathrm{M}: \mathrm{F}=7.54 \pm 5.50: 3.43 \pm 2.23]$, h-Assoc [M:F $=$ $11.07 \pm 7.04: 3.33 \pm 1.53]$ and $\mathrm{h}-\operatorname{Prof}[\mathrm{M}: \mathrm{F}=26.95 \pm 15.65: 24.00$ $\pm 13.11]$.

Conclusion: We present a unified picture of academic spine surgeon publishing productivity. Results may be used for benchmark purposes, for residency program self-assessment or to aid those entering residency in locating possible academic collaboration. h-index data should be interpreted cautiously given few women at each academic rank.

444 Implications of Anesthetic Approach for the Treatment of Spinal Disc Herniation, Spinal vs General Anesthesia

Nikhil Sharma; Matthew Piazza, MD; William Charles Welch, MD, FACS, FICS; Neil R. Malhotra, MD

Introduction: Health care costs continue to escalate. Treatment approaches to care that have comparable outcomes and complica- tions are increasingly assessed for cost and quality. Efforts to identify components of care to reduce length of stay (LOS) have been ongoing, and in particular, spinal anesthesia (SA), for select lumbar spine cases, has garnered interest as an alternative anesthetic to general anesthesia (GA). While clinical outcomes with spinal anesthesia or general anesthesia have been studied, few authors have looked at the cost-analysis in relation with clinical outcomes.

Methods: We retrospectively analyzed 550 patients who underwent hemilaminotomy for disc herniation and who received either spinal anesthesia $(n=91)$ or general anesthesia $(n=459)$. All clinical and billing data was obtained via the patient's electronic medical records and the hospital's billing database, respectively. We studied outcomes measures in a small prospective pilot cohort $(n=75)$ and assessed quality adjusted life year (QALY) gains.

Results: Direct costs for patients receiving SA were $40 \%$ higher than for patients who received GA $(p<0.0001)$. Further, there was a significant difference in regards to length of stay, where patients receiving SA required care for an additional 1.2 hours, a $27 \%$ increase $(\mathrm{p}<0.0001)$. Patients undergoing SA had more comorbidities $(\mathrm{p}=0.0053)$, specifically diabetes and hypertension. However, metrics of complication including readmission $(p=0.3038)$ and $E R$ visits at 30 days $(p=1.0)$ were no different. Further, in a small pilot group, QALY gain was statistically no different $(n=75, p=0.6708)$.

Conclusion: Both SA and GA exhibit the qualities of a good anesthetic and the utilization of these modalities for lumbar spine surgery is safe and effective. However, this work suggests that SA is associated with increased LOS and higher direct costs.

445 Intra-operative and Peri-operative Complications Associated with Endoscopic Spine Surgery: A Multiinstitutional Case Series

Rajeev D Sen, MD; Gabrielle A White-Dzuro, MD; Jacob James Ruzevick, MD; Choll Kim, MD; Jens-Peter Witt; Albert Edward Telfeian, MD, PhD; Michael Y. Wang, MD, FACS; Christoph Paul Hofstetter, MD, PhD

Introduction: Endoscopic spine surgery constitutes a promising new minimally invasive technique. It aims to minimize tissue injury during the surgical approach while achieving the same goals as traditional open surgery. Additionally, the endoscope provides high resolution off-axis visualization of neural structures allowing for novel motion-sparing approaches. While several meta-analyses suggest that endoscopic spine surgery has similar outcomes compared to minimally invasive techniques with a favorable complication profile, larger multicenter cohort studies are lacking.

Methods: A retrospective review of patients operated on by surgeons within the Endoscopic Spine Study Group (ESSG) from May 2010 to June 2017 was conducted. The ESSG is composed of surgeons from five major academic centers with the resulting database containing 553 endoscopic surgeries performed on 534 patients. Patients had an average age of 57 years (range 15-97; 1.5:1 M:F). Surgeries were performed via an interlaminar or transforaminal endoscopic approach. All major intra- and perioperative surgical complications are reported.

Results: A variety of pathologies throughout the spinal column were treated including disc herniation $(377 ; 68 \%)$, central stenosis $(71 ; 12 \%)$, foraminal stenosis $(68 ; 12 \%)$, and others such as lateral recess stenosis, adjacent segment disease, synovial cysts and discitis $(46 ; 8.3 \%)$. Most cases were treated using a transforaminal approach as opposed to the interlaminar approach $(73.8 \%$ versus $24.8 \%)$ and involved a single segment $(83 \%)$. All surgeries were well tolerated and blood loss was minimal. There were a total of 12 intra- or peri-operative surgical complications: 6 dural tears $(1.1 \%), 2$ postoperative hematomas $(0.36 \%), 2$ patients developed complex pain 
syndrome $(0.36 \%)$ and 2 disc re-herniations within 10 days $(0.53 \%)$.

Conclusion: Endoscopic spine surgery is a novel surgical technique that allows for treatment of a variety of common spinal pathologies. Our ESSG registry suggests that intra and peri-operative surgical complication rates and severity compare favorably with traditional open and minimally invasive spinal surgery.

446 Clinical and Radiologic Outcomes of Thoracolumbar Fusions Using Intraoperative CT Guidance (O-Arm) and Stereotactic Navigation (StealthStation): Analysis in Spinal Trauma Populations

Kyle Anthony Smith, MD; Joseph Domino, MD; Sarah Woodrow, MD; Paul M. Arnold, MD

Introduction: Placement of pedicle screws has become commonplace for thoracolumbar fusions. Suboptimal placement may lead to neurovascular complications and postoperative pain. In the advent of outcomes-based payment, precision and accuracy of pedicle screws is important. Image-guided spinal surgery is commonly used to improve accuracy, particularly for complex anatomy such as ankylosing conditions. We sought to analyze the clinical and radiographic outcomes of posterior thoracolumbar fusions using intraoperative CT-guidance (O-Arm, Medtronic) and stereotactic navigation (SteathStation, Medtronic), specifically in thoracolumbar spinal trauma.

Methods: We retrospectively identified 59 patients undergoing posterior thoracolumbar fusions using intraoperative CT and stereotactic navigation for trauma from 2010 to 2017 at a single institution. Specifically, analysis of pedicle screw accuracy, realignment in setting of trauma, and clinical outcomes were queried. Accuracy was judged in comparison to expected placement. Breach grades included: Grade $1(<2 \mathrm{~mm})$, Grade $2(2-4 \mathrm{~mm})$, Grade 3 (>4mm).

Results: 59 patients were identified having undergone 59 operations (595 pedicle screws). Various degree of injury included chance fracture, burst fracture, and fracture-dislocation. Levels of injury varied throughout the thoracic and lumbar spine. Accurate pedicle screw placement, without cortical breach or anterior breach, was found in $97.6 \%$ (581/595). Breach included: Grade 1 in 10 screws $(1.7 \%)$; Grade 2 in 4 screws $(0.7 \%)$. No neurovascular complications were noted. Alignment was restored in all patients $(n=59 ; 100 \%)$. No revision surgery was performed for misplacement. Overall, patient outcomes were significantly improved at follow-up.

Conclusion: Intraoperative CT-guidance and stereotactic navigation can overcome the difficulty associated with complex anatomy, such as thoracolumbar trauma with malalignment and unpredictable trajectories, and can improve the accuracy of instrumentation. Intraoperative CT can be used with stereotactic guidance or for intraoperative verification of free-hand screw placement with repositioning as needed. CT-guidance maintains the benefit of reduced fluoroscopic exposure and reduced reoperation for screw malalignment.

447 Aggressive Surgical and Intensive Unit Care Correlates with Lower Mortality in Elderly Acute Traumatic Spine Cord Injury

Darryl Lau, MD; Cecilia L. Dalle Ore, B.A.; Phiroz E. Tarapore, MD; Michael C. Huang, MD; Geoffrey T. Manley, MD, PhD; Vineeta Singh, MD; Michael Beattie, PhD; Jacqueline Bresnahan, PhD; Adam Ferguson, PhD; Jason Talbott, MD, PhD; William Whetstone, MD; Sanjay S. Dhall

Introduction: Studies have reported high morbidity and mortality when elderly traumatic spinal cord injury (SCI) patients undergo surgery. This study compares perioperative outcomes of elderly SCI patients who were managed surgically to that of a younger cohort.

Methods: Consecutive adult SCI patients who underwent surgery were retrospectively reviewed from a prospectively-collected single institution database from 2007 to 2017. The cohort was divided into less than 70 and 70 years or older. Outcomes of interest included complication, mortality, intensive care unit stay (ICU), length of stay (LOS), disposition, and neurological status.

Results: A total of 106 patients were included: 63 young (mean 45.8 years, range 18 to 69 years) and 23 elderly (mean 78.4 years, range 70 to 88 years). Both groups were similar in demographics, traumatic attributes, imaging features, and American Spinal Injury Association Impairment Scale (AIS) grade. The elderly group had a higher proportion of cervical SCI $(95.7 \%$ vs. $71.1 \%, \mathrm{p}=0.047)$. Overall, $93.4 \%$ underwent instrumented spinal fixation, and $12.3 \%$ corpectomy. Mean levels fused were 4.8 and levels decompressed were 2.7. Mean ICU stay was 13.1 days, and LOS was 23.0 days. There were no significant differences in ICU or LOS between groups. Overall complication rate was $50.0 \%$, and mortality was $3.8 \%$. Elderly patients experienced a significantly higher complication rate $(73.9 \%$ vs. $43.4 \%, \mathrm{p}=0.010)$, but did not experience a higher rate of any specific type of complication. Mortality was significantly higher in elderly patients $(13.0 \%$ vs. $1.2 \%, \mathrm{p}=0.008)$. There was a significant difference in disposition $(\mathrm{p}=0.029)$; none of the elderly patients were discharged home (0.0\% vs. $18.1 \%)$. Discharge AIS and AIS improvement were similar.

Conclusion: Elderly patients had higher complication and mortality rates and were less likely to be discharged home, but mortality rates have improved compared to prior reports. This may reflect the benefit of emergent surgery and aggressive ICU care.

448 Vascularized Spinous Process Graft Rotated on a Paraspinous Muscle Pedicle for Lumbar Fusion: Technique Description and Early Clinical Experience

Michael Bohl, MD; Kaith K Almefty, MD; Mark C. Preul, MD; Jay D. Turner, MD, PhD; Udaya K. Kakarla, MD; Steve W. Chang, MD

Introduction: Vascularized bone grafts (VBGs) are well described in the literature as having superior osteogenicity, osteoconductivity, and osteoinductivity compared to other graft types, and have been used in high-risk patients to augment arthrodesis. Free-transfer VBGs add additional time, complexity, and morbidity to a surgical operation. Pedicled VBGs, however, are rotated on an intact vascular pedicle and therefore maintain all the benefits of VBG, but avoid many of the challenges and additional morbidity of free-tissue transfer. The purposes of this study are to describe a novel surgical technique for rotating vascularized spinous process into the posterolateral space for augmenting arthrodesis in patients undergoing posterolateral fusion (PLF), and to report the early clinical and radiographic outcomes of patients who have undergone this novel procedure.

Methods: A technique is described for rotating the spinous process into the posterolateral space on an intact vascular pedicle of paraspinal muscle. Early clinical and radiographic outcomes are reported for 4 patients who have undergone this procedure.

Results: Four patients were treated with a single or 2-level PLF combined with posterior, anterior, or lateral interbody fusion and vascularized spinous process graft. Three-month postoperative computed tomography scans demonstrated a dislodged graft in 1 patient and successful arthrodesis in 3 patients. Additional operative time taken for graft harvest and implantation ranged from 22 minutes for the first patient to 6 minutes for the fourth patient.

Conclusion: Rotation of vascularized spinous process graft 
for augmentation of posterolateral arthrodesis in the lumbar spine appears to be a safe and effective surgical technique that results in successful arthrodesis in as little as 3 months. This technique is expected to add little additional time or morbidity to the traditional lumbar PLF as it requires no separate incision or additional bone removal.

449 The Barrow Innovation Center Case Series: Early Clinical Experience with a Novel Surgical Instrument Used to Prevent Intraoperative Spinal Cord Injuries

Michael Bohl, MD; Jacob F Baranoski, MD, BS; Daniel Sexton; Peter Nakaji, MD; Laura A. Snyder, MD; Udaya K. Kakarla, MD; Randall W. Porter, MD

Introduction: The Barrow Innovation Center (BIC) is an educational program in medical innovation that enables residents to identify problems in patient care and to rapidly develop and implement solutions to these problems. Residents involved in this program noted an elevated risk of iatrogenic spinal cord injury during posterior cervical and thoracic decompression and fixation procedures; a case report is provided that demonstrates this risk.

Methods: Solutions to this problem were developed through the BIC via an iterative process of prototype creation, cadaveric testing, and redesign. Patent law students partnered with the BIC wrote and filed a provisional patent protecting the novel prototype designs and foreseen device embodiments developed by the residents.

Results: The concept of a protective shield for the spinal cord was developed, and within only 6 weeks the devices were provisionally patented and used in the operating room. These devices were named Myeloshields.

Conclusion: Initial clinical experience indicates that the Myeloshield can be used without impeding the flow of surgery and has the potential to prevent iatrogenic spinal cord injury; this experience is presented through 2 case reports demonstrating the use of Myeloshields in the operating room. This report also demonstrates how programs like the BIC can provide neurosurgery residents with a unique educational experience in medical device innovation and intellectual property development and can serve as an avenue of surgical quality improvement and problem solving.

450 Cortical Trajectory TLIFs Associated with Less Blood Loss and Better Disposition than Pedicle Trajectory and Posterolateral Fusions: A Retrospective Cohort Study

James G Malcolm, MD, PhD; Michael Kevin Moore; Faiz U. Ahmad, MD, MCh; Daniel Refai, MD

Introduction: The cortical screw trajectory with its medial approach is believed to require a smaller incision and less lateral tissue dissection resulting in less blood loss and faster healing; however, this has not yet been confirmed in clinical studies. Our objective is to compare cortical screw TLIFs (CS), traditional pedicle screw TLIFs (PS), and posterolateral fusion without interbody (PLF) testing for differences in operative characteristics and complications.

Methods: Retrospective cohort study (CS, PS, PLF) looking at patients who underwent lumbar fusion with one or two levels only. Extracted data included demographics, estimated blood loss, operative time, length of stay, disposition, and complications within the perioperative, 30 day, and 90 day periods. Cohorts were compared using nonparametric Wilcoxon and Fisher exact tests correcting for multiple comparisons.

Results: Total of 119 patients (44 CS, 36 PS, 39 PLF) with average age $62( \pm 10.3), 72$ females, 47 males, 88 one-level, 31 two-level, and 90-day followup for 102 (86\%) of these patients. CS had significantly less average blood loss $(225 \mathrm{cc})$ than either PLF $(400, p<0.0001)$ and PS $(426, p<0.0001)$. PS had significantly longer average OR time $(260 \mathrm{~min})$ than either CS $(215, \mathrm{p}=0.0023)$ or PLF $(213, \mathrm{p}=0.0008)$. There was no significant difference in length of stay. There were significant differences in disposition for each cohort $(\mathrm{p}=0.0387)$ between home without needs (CS 36\%, PS $19 \%$, PLF $13 \%$ ); home with physical therapy (CS 52\%, PS $61 \%$, PLF 56\%); acute rehab (CS 2\%); and subacute rehab (CS 9\%, PS $19 \%$, PLF $31 \%$ ). There were no significant differences in any of overall complications, perioperative, 30day, 90day, durotomy, or wound healing issues.

Conclusion: The cortical screw trajectory is associated with less blood loss, reduced OR time, more patients returning home without the need of physical therapy, and no difference in complications. This suggests that CS TLIFs may be a less costly alternative for lumbar spine fusion.

451 The History of Neurosurgery at the Medical College of Wisconsin and The Nation's First Spine Fellowship

\section{Andrew Montoure; Marjorie C. Wang, MD, MPH}

Introduction: The Department of Neurosurgery at the Medical College of Wisconsin was born out of the dreams of a few determined leaders, passionate about the field and about what it means to be a neurosurgeon. Neurosurgeons are often known and remembered for their strong personalities; without the likes of Harvey Cushing, Ernest Sachs, Henry Schwartz, Charles Drake and many more, we would not be practicing in the greatest field of medicine there is today. Just as an individual can change the world around them, a Department can grow from the dreams of a few determined and strong personalities; changing the very idea of what it means to become a neurosurgeon and forever changing the field of spinal surgery.

Methods: A historical review of local news articles, departmental historical records, first-hand accounts, and personal memoirs.

Results: Developing the nation's first spine fellowship is an intriguing tale of how a de novo neurosurgical program rose to the forefront of the spinal world with the dedication and drive of Dr. Sanford J. Larson and his colleagues; leaving a lasting impact on several of today's leading spine surgeons with 48 graduated spine fellows, $60 \%$ of those taking academic positions across the country.

Conclusion: A spinal surgeons legacy is based not on what they did but how they did it. The will strength of the leadership and passion for neurosurgery is how an extraordinary group of strong willed individuals turned something from scratch into a major neurosurgical center and leader in spine care. Neurosurgery is a calling which requires immense dedication, heart, and hard workall of which were made a priority here at MCW throughout the years. What started with the engaging personalities of our founding leaders continues through the research, leaders, and trainees of the past, present, and future.

452 Effect of Surgical Setting (Tertiary vs. Community Hospitals) on the Cost of Posterior Cervical Spine Procedures

Heath P Gould, BS; Joseph E Tanenbaum, BA; Kelsey C Goon; Emily Hu, BA; Colin Haines; Thomas E. Mroz, MD; Michael P. Steinmetz, MD; Don K Moore

Introduction: Laminectomy with fusion (LF) has demonstrated clinical benefit for degenerative cervical myelopathy, but the impact of surgical setting on the cost of these procedures remains unclear. We sought to evaluate the cost of LF surgeries performed 
at a large tertiary-care hospital (TH) compared to those performed at smaller community hospitals (CHs).

Methods: A retrospective cohort study was conducted among patients undergoing posterior cervical decompression in 2014 and 2015. CPT codes were used to identify all LF procedures performed in a single healthcare system during this time period. Outcome measures included direct cost, mean length of stay (LOS), and the number of patients discharged to a facility postoperatively.

Results: 209 patients were eligible for inclusion; 156 patients underwent $\mathrm{LF}$ at the $\mathrm{TH}(75 \%)$, while 53 underwent the same procedure at a $\mathrm{CH}(25 \%)$. There were no differences between the two cohorts with regard to age, gender, and marital status. However, a greater proportion of patients treated at the $\mathrm{TH}$ identified as Caucasian compared to those treated at a $\mathrm{CH}(\mathrm{p}<0.05)$. Patients treated at the TH also had a significantly lower BMI $(\mathrm{p}<0.05)$ and marginally lower Charlson Comorbidity Index relative to their $\mathrm{CH}$-treated counterparts. Interestingly, the average cost of performing LF was still $123 \%$ higher at the TH compared to the $\mathrm{CHs}$ $(p<0.001)$. Similarly, the average LOS was significantly longer at the TH $(\mathrm{p}<0.001)$, though this increase in LOS did not translate to any difference in discharge disposition between the two groups.

Conclusion: Despite indications that patients undergoing surgery at the TH may have been healthier at baseline, the cost of LF was higher at the TH compared to the CHs in the same health system. We therefore conclude that surgical setting is a key determinant of the cost and hospital-reported outcomes associated with posterior cervical decompression.

453 Improved Cosmetic Outcome with use of Bilateral Paraspinal Muscle Flap Closure following Cervical Laminectomy and Fusion

John Kenneth Houten, MD; Neil Haranhalli, MD, BA; Daniel Komlos, MD, PhD; Gila R Weinstein, MD

Introduction: Cervical laminectomy and fusion (CLF) research has focused upon neurologic outcome and fusion rate, and poor cosmetic results are rarely considered in assessing surgical complications. Atrophy from muscle denervation and posterior bone loss may lead to a sunken appearance of the back of the neck; and relative tension tends to form wide, unsightly scars. Paraspinal muscle flap elevation to augment midline tissue is routinely employed by plastic surgeons for closure of wound infection and dehiscence. We hypothesize routine use of paraspinal muscle flaps will improve cosmetic results of CLF and possibly reduce incidence of wound dehiscence and infection.

Methods: Data was retrospectively collected for a twelveyear period for patients undergoing CLF including demographic and clinical characteristics and spinal levels addressed at surgery. During the study period, a paraspinal muscle flap closure technique was adopted, initially for patients harboring risk factors for wound complication, and, ultimately, for all cases. Wounds were inspected for scar width and depth using a scale devised to categorize the posterior neck contour. Minimum follow up was 12 months.

Results: Of 159 patients, wound evaluations were performed in 94 , of which 34 had paraspinal muscle flap closure. There were no differences in age, sex, body mass index, mJOA scores, diabetes status, or number of spinal levels treated. Mean follow up was $18.6(12-48)$ and 49.8 (12-130) months in the muscle flap and the conventional closure groups respectively; and contour scores were 1.20 vs. $2.65(\mathrm{p}<.00001)$ and the scar width was 2.8 v $4.9 \mathrm{~mm}$ $(\mathrm{p}<.0001)$. No patient had a wound complication in the muscle flap group and four (7\%) in the conventional closure group.

Conclusion: Bilateral paraspinal muscle flap closure of CLF resulted in improved cosmetic appearance in terms of wound contour and scar width. Further investigation is needed to determine if the technique also decreases wound infection and dehiscence rates.

454 Prediction of Anterior vs. Posterior Surgical Approach for Degenerative Cervical Myelopathy Based on MRI Pathology: Analysis of a Global Cohort

Aria Nouri, MD, MSc; Allan R. Martin, BASc, MD; So Kato; Christopher D. Witiw, MD; Anick Nater, MD; Lindsay Tetreault, Bsc; Carlo Santaguida, MD, FRCSC; David Gimbel, MD; Rani Nasser, MD; Joseph S. Cheng, MD, MS; Michael G. Fehlings, MD, PhD, FRCS(C), FACS

Introduction: Degenerative Cervical Myelopathy (DCM) is the most common cause of spinal cord compression (SCC) and impairment. Surgical treatment is usually undertaken but the decision between an anterior or posterior approach as the optimal choice is often controversial. To better understand this surgicaldecision making process, we analyzed imaging data from two prospective multicenter studies.

Methods: MRIs of 458 patients were analyzed for pathologic features, characteristics of SCC, sagittal alignment using the modified K-line, and signal changes on T1-weighted (T1WI) and T2-weighted images (T2WI). Clinical severity was measured with modified Japanese Orthopedic Association score (mJOA). Fisher exact tests and multivariate logistic regression were used to assess relationships between clinical/MRI features and anterior (A), posterior (P), or combined (AP) surgical approach.

Results: Operative approach was $\mathrm{A}=265, \mathrm{P}=184$, and $\mathrm{AP}=9$. Anterior surgery was favored with lower age, $\mathrm{mJOA}=15$, singlelevel disc pathology, anterior-only SCC, and kyphosis, while posterior approach was favored in South America or with multilevel spondylosis, ligamentum flavum enlargement, spondylolisthesis, SCC at or above C4-5, SCC at or below C6-7, more levels with SCC, T2WI hyperintensity, and more levels with T2WI hyperintensity (all $\mathrm{p}<0.05)$. In multivariate analysis, levels with SCC $(p<0.001)$, age $(p<0.001)$, South American region $(p<0.001)$, mJOA=15 $(\mathrm{p}=0.008)$, kyphosis $(\mathrm{p}=0.02)$, and maximal SCC at or above $\mathrm{C} 4-5(\mathrm{p}=0.05)$ were significant independent predictors. Models based on clinical and MRI factors predicted A vs. P approach with $78 \%$ and $80 \%$ accuracy, respectively, whereas a combined model achieved $87 \%$ accuracy.

Conclusion: Clinical and MRI factors are independently able to predict surgical approach in DCM, and a combined model has excellent predictive capacity. While the predictive utility of specific MRI factors on surgical approach corroborate expert opinion, this study has also provided information regarding the magnitude of their influence. These findings may be used in conjunction with machine learning to develop surgical treatment algorithms for DCM.

455 The Relationship Between Improvements in Myelopathy and Sagittal Realignment in Cervical Deformity Surgery Outcomes

Peter G Passias, MD; Samantha R. Horn; Cole Bortz BA; Subaraman Ramchandran, MBBS, MS, (orth); Douglas C. Burton, MD; Themistocles Protopsaltis, MD; Renaud Lafage; Virginie Lafage, PhD; Bassel G. Diebo, MD; Gregory W, Poorman; Frank Segreto; Muhammad Burhan Ud Din Janjua, MD; Justin S. Smith, $\mathrm{MD}, \mathrm{PhD}$

Introduction: CD correction involves radiographic malalignment correction and procedures to improve motor function and pain. It is unknown whether alignment or myelopathy improve- 
ment drives patient outcomes. The aim of this study was to determine if alignment or myelopathy improvement drives patient outcomes following cervical deformity(CD) corrective surgery.

Methods: Retrospective review of multicenter database. Inclusion: $C D$ patients with baseline/1-year radiographic and outcome scores. Cervical alignment improvement was defined by improvement in Ames CD modifiers. mJOA improvement was defined as: mild[15-17], moderate[12-14], severe[<12]. Patient groups included those who only improved in alignment, those who only improved in mJOA, those who improved in both, and those who didn't improve. Changes in quality-of-life scores (NDI, EQ-5D, mJOA) were evaluated between groups.

Results: 70 patients $(62 \mathrm{yrs}, 51 \% \mathrm{~F})$ were included. Overall pre-operative mJOA score was $13.04 \pm 2.35$. At baseline, $21(30 \%)$ patients had mild myelopathy, 33(47\%) moderate, and $16(23 \%)$ severe. $31 / 70(44 \%)$ patients improved in mJOA and $13(18.6 \%)$ met $1 Y$ mJOA MCID. Distribution of improvement groups: 16/70(23\%) alignment-only improvement, 13(19\%) myelopathy-only improvement, $18(26 \%)$ alignment and myelopathy improvement, $23(33 \%)$ no improvement. EQ-5D improved in $11 / 16(69 \%)$ alignmentonly patients, 11/18(61\%) myelopathy/alignment improvement, $13 / 13(100 \%)$ myelopathy-only, and 10/23(44\%) no myelopathy/ alignment improvement. There weren't differences in decompression, baseline alignment, mJOA, EQ-5D or NDI between groups. Patients who improved only in myelopathy showed significant differences in baseline-1Y EQ-5D(baseline:0.74,1Y:0.83,p<0.001). One-year C2-S1 SVA (mJOA r=-0.424,p=0.002; EQ-5D r=$0.261, \mathrm{p}=0.050$; NDI $\mathrm{r}=0.321, \mathrm{p}=0.015)$ and C7-S1 SVA (mJOA r $=$ $0.494, \mathrm{p}<0.001$; EQ-5D r=-0.284,p=0.031; NDI $\mathrm{r}=0.334, \mathrm{p}=0.010$ ) were correlated with improvement in HRQLs.

Conclusion: ollowing CD-corrective surgery, improvements in myelopathy symptoms and functional score were associated with superior 1-year patient-reported outcomes. Although there were no relationships between cervical-specific sagittal parameters and patient outcomes, global parameters of C2-S1 SVA and C7-S1 SVA showed significant correlations with overall 1-year mJOA, EQ-5D, and NDI. These results highlight myelopathy improvement as a key driver of patient-reported outcomes, and confirm the importance of sagittal alignment in CD patients.

456 Identifying Sources of Improvement of Axial Pain in Corrective Cervical Deformity Surgery

Gregory W. Poorman; Peter G Passias, MD; Samantha R. Horn; Bassel G. Diebo, MD; Frank Segreto; Cole Bortz, BA; Muhammad Burhan Ud Din Janjua, MD; Eric Klineberg, MD; Christopher I. Shaffrey MD, FACS; Virginie Lafage, PhD; Themistocles Protopsaltis, MD; Christopher P. Ames, MD; Justin S. Smith, MD, PhD; Gregory M. Mundis, MD, Brian J. Neuman, MD; Robert Hart, MD; Douglas C. Burton

Introduction: Identifying factors likely to improve symptomology following cervical deformity(CD) corrective surgery is key to improving surgical outcomes. This study identifies prognostic variables associated with improvements in neck pain following CD surgery.

Methods: Retrospective review of prospective multicenter database. Included: patients $>18$ meeting one of the following criteria: $\mathrm{C} 2-7 \mathrm{Cobb}$ angle $>10^{\circ}$, coronal Cobb angle $>10^{\circ}$, C2-C7 sagittal vertical axis $>4 \mathrm{~cm}$ or $\mathrm{T} 1-\mathrm{C} 6>10^{\circ}$, chin-brow vertical angle $>25^{\circ}$. Patients who reached 1-year postoperative MCID(improvement>2.6 NRS Neck; >1.2 NRS Back) for pain improvement were compared to those who didn't. Two groups, those who met MCID and those who didn't(NOT group), were constructed with equal baseline Numeric Rating Scale(NRS) for neck pain by propensity score matching for pain. T-tests assessed clinical and deformity data, comparing how treatments that improved neck pain and back pain differed from those that didn't.

Results: 122 patients $(61.1 \mathrm{yrs}, 60.4 \% \mathrm{~F}$,mean BMI:30.1) presented with average baseline neck pain score of 7.0. Patients improved in neck pain at 3-months(4.4), 6-months(4.0), 1 -year(4.4,all $\mathrm{p}<0.05)$. $51.1 \%$ of patients reached 1-year MCID for neck pain. 33 MCID group patients were compared with 33 NOT group, matching for baseline NRS neck pain(neck MCID group:7.0; NOT:6.8,p=0.72). NOT patients had more posterior approach (MCID:37.5\%; NOT:62.5\%,p=0.04) and revision surgeries(MCID:25.8\%; NOT:53.1\%, $\mathrm{p}=0.03$ ), but similar levels fused(MCID:7.2 levels; NOT:8.1,p=0.32). Both groups had similar CD, measured by TS-CL, C2-C7 lordosis and cSVA(all $\mathrm{p}>0.05)$. MCID patients had large lumbar deformities: Pelvic Tilt(MCID:22.3; NOT:16.6,p<0.05) and PI-LL(MCID:5.4; NOT:$4.5, \mathrm{p}=0.02)$. Evaluating secondary back pain change in CD corrections, patients presented with an average baseline back pain score of 5.2. Patients improved at 3-months(4.6), 6-months(4.6), and 1 -Year(4.8) intervals(all $\mathrm{p}<0.05$ ). $30.6 \%$ of patients met 1 -year back pain MCID. There wasn't overlap between patients improving in back pain and neck pain(MCID:9.4\%; NOT: $21.9 \%, \mathrm{p}=0.30$ ).

Conclusion: Revision and posterior approach are predictors of failure to reach improvement in neck pain. Patients also improved in lower back pain following cervical realignment, despite primary cervical pathology.

457 Predicting the Occurrence of Complications Following Corrective Cervical Deformity Surgery: Analysis of a Prospective Multicenter Database Using Predictive Analytics

Peter G Passias, MD; Cheongeun Oh; Samantha R. Horn; Han Jo Kim, MD; D. Kojo Hamilton, MD; Daniel M. Sciubba, MD; Brian J Neuman, MD; Aaron James Buckland, MBBS, FRACS; Gregory W, Poorman; Cole Bortz, BA; Frank Segreto; Muhammad Burhan Ud Din Janjua, MD; Themistocles Protopsaltis, MD; Eric Klineberg, MD; Christopher P. Ames, MD; Justin S. Smith, MD, $\mathrm{PhD}$; Virginie Lafage Phd; International Spine Study Group

Introduction: Multivariate regression models may be useful in determining the relative impact of patient and clinical predictors for adverse outcomes. Cervical deformity(CD) surgical patients are growing in number, but remain under-studied in the literature. The purpose of this study was to develop a predictive model to describe factors that predict surgical and medical complications in cervical deformity surgeries.

Methods: Retrospective review of $\mathrm{CD}$ database. $\mathrm{CD}$ ws defined as at least one of the following: $\mathrm{C} 2-\mathrm{C} 7 \mathrm{Cobb}>10^{\circ}, \mathrm{CL}>10^{\circ}$, $\mathrm{cSVA}>4 \mathrm{~cm}, \mathrm{CBVA}>25^{\circ}$. Medical complications:cardiopulmonary, dysphagia, GI/GU, neurologic, respiratory,; surgical:surgical site infection, dural tear, wound dehiscence, radiographic/implant failure. Univariable and multiple logistic regression analyses assessed each independent variable with the development of any, medical and surgical complications.

Results: 123 patients underwent CD-corrective surgery(60.6 years,60.8\%F, 8.1 levels fused, 297min op-time, 776cc EBL). A total of $93(75.6 \%)$ complications were reported up to 1-year. $51(41.5 \%)$ patients experienced a medical complication(cardiac, dysphagia, neurologic most common) and 73(59.3\%) had a surgical complication(infection, dural tear, DJK). Corpectomy(OR:0.54, CI:0.30-0.98), baseline cSVA(OR: 1.02,CI:1.00-1.04), McGregor's slope(OR:1.03,CI:1.00-1.03) increased chances of medical complications. Higher blood loss(OR:1.434,CI:1.086-1.894) and baseline global SVA (OR:1.93,CI:1.04-3.59) increased surgical complications risk. Overall complication was predicted with high accuracy $(\mathrm{AUC}=0.80)$ by male gender, higher baseline EQ5D pain, lower anxiety/depression scores, and higher cervical and 
global SVA. A medical complication was predicted by male gender, baseline mJOA score, hand numbness, and cervical SVA(AUC=0.770). Surgical complications were predicted by higher estimated blood loss, lower anxiety scores, and larger SVA(AUC $=0.739)$.

Conclusion: $75.6 \%$ of cervical deformity surgical patients sustained a complication. While the most reliable predictor of the occurrence of a complication involved a cluster of risk factors, a radiographic baseline sagittal parameter of cervical SVA was the strongest isolated predictor for complications across categories. Although these findings are specific to a cervical deformity population with moderate to severe deformities, collectively they can be utilized for pre-operative risk assessment and patient education.

458 Propensity Matched Comparison of Outcomes and Cost After Macroscopic and Microscopic ACDF Using a National Longitudinal Database

Allen L Ho, MD; Arjun Vivek Pendharkar, MD; Paymon Rezaii; David Arnold Purger, MD; Eric S Sussman, MD; Anand Veeravagu, MD; John K. Ratliff, MD, FACS; Atman Desai, MD, MA

Introduction: There has been considerable debate about the utility of the operating microscope in anterior cervical discectomy fusion (ACDF) and its effect on outcomes and cost.

Methods: A commercially available longitudinal database (MarketScan) was used to identify patients undergoing discectomy with or without microscope between 2007-2015. Propensity matching was performed to normalize differences between demographics and comorbidities in the two cohorts. Outcomes, complications and cost were subsequently analyzed with multivariate logistic regression.

Results: A total of 91605 patients were identified for the 'macroscopic' group, while 9669 patients were identified for the microscope group. There were no significant differences in postoperative complications between the groups. Length of stay was significantly longer in the macroscopic group compared to microscopic ACDF (1.67 days vs 1.56 days $\mathrm{p}<0.00001)$. Readmission and reoperation rates were not significantly different, but microscopic ACDF patients also had a higher rate of discharge home versus outside facilities compared to macroscopic ACDF (OR 1.61 (1.47-1.77, $\mathrm{p}<0.0001)$. Finally, physician and total charges were higher in the microscopic group $(\$ 8,057$ vs $\$ 6,687, \mathrm{p}<0.0001$ physician; $\$ 36,502$ vs $\$ 34,652$; $p<0.0001$ total), though there was no significant differences in hospital charges.

Conclusion: The present study suggests that the use of the operating microscope in ACDF is associated with decreased length of stay and higher rate of discharge home. However, the microscope was associated with higher physician and total cost.

459 A Comparison of Clinical Outcome Between Standalone Zero-profile Implant Versus Cervical Plate Fixation in Single and Multilevel Anterior Cervical Discectomy and Fusion: An Institutional Analysis of 166 Patients

Samuel Aramis Cornelio Sommaruga, MD; Joaquin Q CamaraQuintana; Aria Nouri, MD, MSc; Justin Virojanapa, DO; Luis Kolb, MD; Kishan Patel; Julio D. Montejo, BA; Patrick Tomak, MD; Khalid M. Abbed, MD; Joseph S. Cheng, MD, MS

Introduction: The stand-alone (SA) zero-profile implant has become an attractive alternative to cervical plating (CP) in anterior cervical discectomy and fusion (ACDF) for the treatment of degenerative cervical myelopathy (DCM) and degenerative cervical radiculopathy (DCR).
Methods: We conducted a retrospective analysis of a prospective cohort of consecutive patients with DCM and DCR who underwent ACDF with SA or CP from 2013 to 2016. Outcome measurements included: Bazaz dysphagia, post-operative steroid use, and Nurick grade at last follow-up. Neurological outcome was dichotomized with the Nurick grade. Assessments of differences between outcomes were performed using the Fisher exact test for categorical variables and the Mann-Whitney $U$ test for continuous variables. A multivariate logistic regression model was constructed using variables that reached the predetermined significance level in the univariate analysis.

Results: Of the 166 patients (92 females), 84 (51\%) presented with radiculopathy, $36(21 \%)$ with myelopathy, $35(21 \%)$ with myeloradiculopathy and $11(7 \%)$ with intractable pain. The two groups had a statistically significant ( $\mathrm{p}$-value $=2.5 \times 10-9)$ difference time of surgery, 195 (70) minutes for CP vs. 127 (46) minutes for SA. The SA group had a statistically significant ( $\mathrm{p}$-value = 0.024) shorter length of stay (1.51 day) than the CP group (2.12 days). Four patients reported mild dysphagia. However, all 4 patients recovered at their last follow-up. Nurick grade improved in 140 patients while 26 patients showed no change or worse Nurick grade. Multivariate analysis revealed no statistically significant independent predictors of outcome between SA or CP.

Conclusion: This study is one of the largest studies comparing SA and CP. Our analysis demonstrated that there were no statistically significant differences in rates of dysphagia and neurological outcome between CP and SA. However, it has been shown here that length of hospital stay and the operative time was shorter in patients with SA

460 T1 Slope is a Potent Predictor of Subsidence in Anterior Cervical Discectomy and Fusion with Stand-alone Cages

Dong Wuk Son; Geun Sung Song; Sang Weon Lee; Jun Seok Lee, M.D.

Introduction: Preoperative parameters including the T1 slope (T1S) and C2-7 sagittal vertical axis (SVA) have been recognized as predictors of kyphosis after laminoplasty, which is accompanied by posterior neck muscle damage. The importance of preoperative parameters has been under-estimated in anterior cervical discectomy and fusion (ACDF) because there is no posterior neck muscle damage. We aimed to determine whether postoperative subsidence and pseudarthrosis could be predicted according to specific parameters on preoperative plain radiographs.

Methods: We retrospectively analyzed 41 consecutive patients (male:female, 22:19; mean age, 51.15 +/- 9.25 years) who underwent ACDF with a stand-alone polyether-ether-ketone (PEEK) cage ( $>1$ year follow-up). Parameters including SVA, T1S, segmental angle and range of motion (ROM), C2-7 cervical angle and ROM, and segmental inter-spinous distance were measured on preoperative plain radiographs. Risk factors of subsidence and pseudarthrosis were determined using multivariate logistic regression.

Results: Fifty-five segments (27 single-segment and 14 twosegment fusions) were included. The subsidence and pseudarthrosis rates based on the number of segments were $36.4 \%$ and $29.1 \%$, respectively. Demographic data and fusion level were unrelated to subsidence. A greater T1S was associated with a lower risk of subsidence $(\mathrm{p}=0.017$, odds ratio $=0.206)$. A cutoff value of $\mathrm{T} 1 \mathrm{~S}<28^{\circ}$ significantly predicted subsidence (sensitivity: $70 \%$, specificity: $68.6 \%$ ). There were no preoperative predictors of pseudarthrosis except old age.

Conclusion: A lower T1S $\left(\mathrm{T} 1 \mathrm{~S}<28^{\circ}\right)$ is a risk factor of subsidence following ACDF. Surgeons need to be aware of this risk factor and should consider various supportive procedures to reduce the subsidence rates for such cases. 
461 Early Decompression is Associated with Significantly Higher Rate of ASIA Impairment Scale Conversion After Cervical Spinal Cord Injury

John Frederick Burke, MD, PhD; Anthony Michael DiGiorgio, DO, MHA; John K. Yue, BA; Ethan A. Winkler, MD, PhD; Laura Benjamin Ngwenya, MD, PhD; Phiroz E. Tarapore, MD; Michael C. Huang, MD; Geoffrey T. Manley, MD, PhD; Sanjay S. Dhall, MD

Introduction: Cervical Spinal Cord Injury (SCI) is a devastating condition with very few treatment options. The most devastating outcome is paralysis the lack of motor function below the level of the injury (AIS grade A or B). Early surgical decompression of the cervical spine after injury has been shown to improve outcomes, however it unknown if early surgical decompression is able to convert AIS A/B injuries to lesser injuries.

Methods: We collected data from 48 patients with cervical SCI. Based on the time to surgical decompression, we grouped patients into a ultra-early group (decompression within 12 hours of presentation), early group (within 12-24 hours), and a late group ( $>24$ hours). We compared the improvement in AIS grade from admission to discharge in each patient group, controlling for confounding factors such injury severity and age.

Results: After controlling for confounding factors, $46.4 \%$ of high-grade patients (AIS A/B) who received surgery less than 24 hours converted to lesser degrees, compared to $14.3 \%$ of patients who received surgery after 24 hours $(42 \%, \mathrm{p}=0.006)$. In addition, ultra-early patients (< 12 hours) improved on average 1.3 AIS grades from admission to discharge, compared to 0.5 in the early group $(\mathrm{T} 46=2.42, \mathrm{p}=0.02)$.

Conclusion: These data suggest that ultra-early $(<12 \mathrm{hrs})$ surgery is not only superior in terms of neurologic recovery.

462 Complications of Crossing the Cervicothoracic Junction in Patients Undergoing Laminectomy and Fusion

Brooke Kennamer; Yakov Gologorsky, MD; Marc Arginteanu; Frank M. Moore, MD; Alfred A. Steinberger, MD; Kevin C. Yao, MD

Introduction: This study sought to determine if there is a difference in revision rate, neck disability index score and cervical alignment in patients undergoing multilevel posterior cervical fusion at C5-6, C7, T1, or T2-4.

Methods: This study examined a dataset of patients who underwent a three or more level posterior cervical decompression and fusion terminating at $\mathrm{C} 4$ to $\mathrm{T} 4$ with at least 6 months of clinical follow-up. The dataset was gathered from a single practice. Patients were separated into four cohorts on the basis of the caudal level of the fusion: C5-6, C7, T1, and T2-4. Several variables were collected: revision rate, neck disability index score, sagittal vertical axis and cervical lordosis. Correlations between the cohorts and these dependent variables was examined using Kruskal-Wallis and Fisher exact tests.

Results: The overall revision rate is $6.7 \%(n=15)$. There is no statistically significant difference in the revision rate identified between fusions terminating at C5-C6, C7, T1, T2-4 $(2.6 \%, 5.7 \%$, $14.6 \%$, and $0 \%$ respectively). Bivariate linear regression was used to account for additional variables in patients whose construct terminated at C5-C6, C7, T1, and T2-T4. There is a statistically significant relationship between a decreased neck disability index and increased cervical lordosis $(\mathrm{p}=0.01)$. There is also a statistically significant relationship between revision rate and cervical lordosis and sagittal vertical axis $(\mathrm{p}=0.004)$.
Conclusion: The challenges of operating at the cervicothoracic junction are widely known yet the selection of the most appropriate caudad level for a multilevel posterior cervical decompression and fusion has yet to be fully understood. This study establishes that constructs should end at $\mathrm{C} 7$ and above as there is an increased revision rate for terminating a construct at $\mathrm{T} 1(\mathrm{p}=0.04)$. There is also a statistically significant difference in increased postoperative cervical lordosis and a decreased neck disability scores $(\mathrm{p}=0.01)$.

463 Effect of Cervical Deformity Correction on Spinal Canal Cross-sectional Area and Stenosis

Peter G Passias, MD; Gregory W. Poorman; Charles Wang; Themistocles Protopsaltis, MD; Christopher I. Shaffrey, MD, FACS; Robert Hart, MD; Virginie Lafage, PhD; Bassel G. Diebo, MD; Justin S. Smith, MD, PhD; Frank Segreto; Samantha R. Horn; Cole Bortz, BA; Muhamma

Introduction: Controversy exists over the efficacy of decompression versus realignment in cervical deformity patients with stenosis. Despite indications that cervical kyphosis effects spinal cord compression, the effects of realignment on canal crosssectional area is not well understood.

Methods: Retrospective analysis of a prospective cervical deformity database. Cervical deformity was defined as: cervical-kyphosis (C2-7 Cobb-angle $>10^{\circ}$ ), cervical-scoliosis (coronal-Cobb-angle $>10^{\circ}$ ), positive-cervical-sagittal-imbalance (C2-C7 sagittal-verticalaxis $>4 \mathrm{~cm}$ or $\mathrm{TS}-\mathrm{CL}>10^{\circ}$ ), or horizontal-gaze-impairment (chinbrow-vertical-angle $>25^{\circ}$ ). Canal-area was assessed from C2-C7/T1 in patients with pre-operative and 1-year MRI's. Stenotic vertebral levels were measured using Pavlov's ratio from C2-T1. Postoperative changes in canal-area and number of stenotic levels were compared between variant operative techniques and cervical deformity classifications using t-tests. Associations between radiographic alignment, myelopathy scores, canal area, and stenotic levels were assessed using bivariate correlation tests.

Results: 14 patients (age 60.2 years, BMI: 32.1, Gender: 8 female) were included. 11 received an osteotomy, 8 a decompression procedure, 6 a posterior-only-approach while the remaining received anterior-then-posterior-approach. Patients with cervical spine deformity apices had less baseline canal-area (cervicalapex: $231.6 \mathrm{~mm} 2$ vs. lower-apex: $303.6 \mathrm{~mm} 2, \mathrm{p}=0.022$ ) and more stenotic levels (cervical-apex: 6.0 vs. lower-apex: $3.4, \mathrm{p}=0.049$ ). Patients presented with an average canal-area of $272.4 \mathrm{~mm} 2$ and 4.6 stenotic levels. At 1-year, average canal-area was $343.8 \mathrm{~mm} 2$ (29\% increase), average stenotic levels was 2.9 (37\% decrease), and differences in canal-area and stenotic levels disappeared between cervical and lower apex deformities $(\mathrm{P}>0.05)$. There was no significant relationship between decompressive techniques and area increases (with decompression: $+75 \mathrm{~mm} 2$, w/o: $+64 \mathrm{~mm} 2$, $\mathrm{p}=0.591)$. Increased canal area and improved myelopathy scores were insignificantly correlated $(\mathrm{r}: 0.509, \mathrm{p}=0.381)$. Increased cervical-lordosis correlated with change in stenotic levels (r:0.752, $\mathrm{p}=0.005)$ and increased canal area (r:0.640, $\mathrm{p}=0.025)$.

Conclusion: This analysis shows an increase in canal area and correction of stenosis in cervical deformity patients. Realignment resulted in increased canal area irrespective of whether or not a decompression procedure was performed.

464 Relationship Between Surgical Site Drains and Reoperation for Wound-related Complications Following Posterior Cervical Spine Surgery: A Multicenter Retrospective Study

Daniel Blakeley Herrick, BA; Ron I. Riesenburger, MD; Jeffrey Florman, MD; William F Lavelle, MD; Joseph Tanenbaum, BA; 
Thomas Mroz; Shane Burke, MD; Marie Roguski, MD; Swamy Kurra; Sagar Vallabh; Eitan Fleischman; Marc Mankarious

Introduction: Use of surgical site drains following posterior cervical spine surgery is variable, and its impact on outcomes remains controversial. Studies in the lumbar spine suggest drains are not associated with reduction of reoperations for wound infection or hematoma. There is a paucity of studies examining this relationship in the cervical spine where hematomas and infections can have severe consequences.

Methods: This study is a multicenter retrospective review of 1,799 consecutive patients undergoing posterior cervical decompression with instrumentation at four tertiary care centers treated between 2004-2016. Demographic and perioperative data were analyzed for associations with drain placement and return to the operating room.

Results: Of 1,799 patients, 1,180 (65.6\%) had a drain placed. Multivariate logistic regression analysis identified history of diabetes (OR 1.37, $\mathrm{P}=0.03$ ) and total number of levels operated (OR 1.32, $\mathrm{P}<0.001)$ as independent predictors of drain placement. Rates of reoperation for any surgical site complication were not different between the drain and no-drain groups $(4.07 \%$ vs. $3.88 \%$, $\mathrm{P}=0.85)$. Similarly, rates of reoperation for surgical site infection $(1.61 \%$ vs. $2.58 \%, \mathrm{P}=0.16)$ or hematoma $(0.68 \%$ vs. $0.48 \%$, $\mathrm{P}=0.62)$ were not different between the drain and no-drain groups. However, after adjusting for history of diabetes and the number of operative levels, patients with drains had significantly lower odds of returning to the operating room for surgical site infection (OR $0.48, \mathrm{P}=0.04$ ) but not for hematoma (OR 1.22, $\mathrm{P}=0.77$ ).

Conclusion: This large study characterizes current practice patterns in the utilization of surgical site drains during posterior cervical decompression and instrumentation. Patients with drains placed did not have lower odds of returning to the operating room for postoperative hematoma. However, our data suggests patients with drains may be less likely to return to the operating room for surgical site infection though the absolute number of infections in the entire population was small limiting the analysis.

465 Comparative Analysis of Changes in Spinal Canal Dimension and Myelopathy Improvement Between Patients with and without Cervical Deformity

Peter G Passias, MD; Gregory W. Poorman; Charles Wang; Shaleen Vira; Cyrus M Jalai, B.A.; Bassel G. Diebo, MD; Cole Bortz, BA; Frank Segreto; Samantha R. Horn; Jared Tishelman; Shenling Wang, MD; Renaud Lafage; Virginie Lafage, PhD

Introduction: For patients with cervical deformity, simple decompression of stenosis may not be sufficient to relieve spinal cord compression and restore neurologic function. No comparison exists between deformity and nondeformity patients in amount of spinal canal area change after realignment or decompression, respectively. The aim of this study was to compare the effects of decompression versus realignment on spinal canal area and functional outcomes in cervical spondylotic myelopathy (CSM) patients.

Methods: Prospective consecutive series. 31 patients with a primary diagnosis of CSM treated with either realignment or decompression procedures and with a minimum follow-up of 1 year were divided into 2 cohorts based on the presence of cervical deformity (CD) and non-cervical deformity (non-D). Cervical deformity was defined as: cervical-kyphosis $\left(>10^{\circ}\right)$, cervical-scoliosis $\left(>10^{\circ}\right)$, cervical-SVA $>4 \mathrm{~cm}$, or horizontal-gaze-impairment (chin-brow-vertical-angle $>25^{\circ}$ ). Preoperative and 1-year MRIs were assessed for spinal canal-area and stenotic vertebral-levels using Pavlov's ratio from C2-T1. Changes in canal-area, number of stenotic-levels, and myelopathy score (mJOA) from baseline to 1 -year between CD and non-D cohorts were assessed using t-tests.

Results: 14 patients with CD(age 60.2 years, BMI: $32.1,54 \%$ female) were compared to 17 non-D patients (age 51.2 years, BMI: $27.7,56 \%$ female). Within the CD cohort, average levels-fused was $8.0,4 / 14$ underwent decompressions, and 8/14 underwent vertebral-body osteotomy. Within the Non-D cohort, average levels-fused was 3.4, 17/17 had decompressions, and none underwent vertebral-body osteotomies. Baseline canal-area was similar across deformity groups: (CD:259.3mm2 vs. non-D:279.1mm2, $\mathrm{p}=0.267$ ). Post-operative change in area was similarly non-different (CD:+75.5mm2 vs. non-D:+46.9mm2, $\mathrm{m}=0.149)$. Patients presented with comparable number of stenotic-levels (CD: 4.7 vs. non-D: $4.2, p=0.484$ ) and postoperative decreases in stenotic-levels (CD: -2.0 vs. non-D: $-1.8, \mathrm{p}=0.807)$. Lastly, mJOA improvement was similar (CD: +0.50 vs. non-D: $+1.8, \mathrm{p}=0.449$ ).

Conclusion: For patients with CSM, realignment of $\mathrm{CD}$ patients offers post-operative improvements of similar caliber to decompressive techniques used by non-D patients.

466 Interobserver Agreement in the Interpretation of Correct Application of Cervical Orthosis after Cervical Fusion

Chad Claus, D.O.; Sean D Krieger, BSc; Doris Tong, MD; Sina Rajamand, DO; Teck Mun Soo; Clifford Michael Houseman, DO

Introduction: The Effectiveness of external cervical orthosis depends on correct collar application. Data on correct cervical collar application is lacking. We sought to measure the level of agreement in interpretation of correct collar application between cervical fusion patients and trained observers.

Methods: We prospectively interviewed consecutive patients who underwent anterior/posterior cervical fusion in two hospitals with multiple surgeons at their 2-9 weeks' postoperative visits from $2 / 1-8 / 1 / 2017$. We excluded personal injuries or patients $<18$-year-old. Patients and trained raters both evaluated the correct application of the Vista cervical collar simultaneously using a cloud-based and secured questionnaire with a 4-point ordinal scale (Fig.1). Patients were blinded to the assessment by raters. Patient and raters evaluated the collar application once. Raters were selected randomly from departmental researchers who were trained using a standardized Vista collar training video produced by Aspen Medical Products. Patients were verbally trained by the nurses/residents on discharge. Assuming null hypothesis with an Intraclass correlation (ICC) coefficient of 0.5 , we need 138 patients to detect a 2 -tail disagreement of 0.2 . Single-rating, absolute-agreement, two-way random-effects model was used. We accepted ICC value of $<0.5$ as poor agreement and $\mathrm{p}<0.05$ as significant.

Results: One hundred sixty-two collar applications were evaluated by the patients themselves and 8 trained raters. There was no replicate observation. Patient characteristics were presented in table 1 . There were 120 (75\%, 95\% CI 0.68-0.8) concordant pairs. The single measure ICC was $0.48(95 \% \mathrm{CI}, 0.35-0.59, \mathrm{P}<0.0005)$.

Conclusion: Our data demonstrated poor agreement between trained observers and patients regarding correct collar application. To accurately evaluate the effect of external cervical orthosis on cervical fusion, the collar needs to be applied correctly. Standardized orthosis application training is recommended.

467 Direct Cost Analysis of 38 Cervical Spinal Deformity Operations Across Two Major Spinal Deformity Centers with Implications for Catastrophic Costs and 90-Day Cost Bundles

Corinna Clio Zygourakis, MD; Justin K. Scheer, MD; 
Seungwon Yoon; Samrat Yeramaneni, MBBS, PhD; Richard A. Hostin, MD; Michael F. O'Brien, MD; Christopher I. Shaffrey, MD, FACS; Justin S. Smith, MD, PhD; Vedat Deviren, MD; Christopher P. Ames, MD

Introduction: The Centers of Medicare and Medicaid Services (CMS) has recently implemented 90-day cost bundles for joint replacement surgery, and taken steps to expand bundled payment programs. To guide bundled payment plans, our goal is to determine 90-day costs associated with cervical spinal deformity across two spinal deformity centers.

Methods: Retrospective evaluation of cervical spinal deformity patients 2013-2016 at two major metropolitan deformity centers (UCSF, Baylor). Inclusion criteria included adult patients with cervical kyphosis $\left(\mathrm{C} 2-7 \mathrm{Cobb}\right.$ angle $\left.>10^{\circ}\right)$ or cervical scoliosis (coronal Cobb able $>10^{\circ}$ ). Direct hospital costs for the index surgery and all visits within the first 90 days after surgery were determined from hospital billing data. Direct costs include surgical supplies/implants, room/care, pharmacy; they exclude indirect costs such as overhead, administration, and utilities.

Results: 38 patients $(25 \mathrm{~F}, 13 \mathrm{M}$; mean age $=60.4 \pm 9.8$ years $)$ underwent cervical deformity fusions. 29 procedures were posterior fusions, 1 was anterior, 7 were anterior-posterior, and 1 was posterior-anterior-posterior. Average levels fused was 9.3 \pm 3.3 . Average direct hospital cost for 90-day period was $\$ 64,480 \pm 23,816$, and was similar between the two institutions $(\$ 62,569$ versus $\$ 70,638)$. 4 of 38 patients $(10.5 \%)$ were readmitted within 90-days, and these re-admission costs constitute the majority of these patients' post-index surgery costs. 2 of 38 $(5.3 \%)$ patients had catastrophic total 90 -day costs, which were greater than two standard deviations above the mean $(>\$ 112,112)$.

Conclusion: Average 90-day direct hospital costs for patients undergoing cervical spinal deformity surgery are $\$ 64,480$. Readmissions and a greater number of operated levels contribute to higher costs; reasonable 90-day cost bundle plans must account for both of these issues. An important limitation is that our analysis includes costs at our main hospital only; it does not include postoperative outpatient medications, physical therapy, rehabilitation, or admissions to outside hospitals. It is therefore imperative that further studies utilizing national databases are performed to understand the full scope of 90-day costs.

468 Tract-specific Volume Loss on High-resolution Spinal MR Imaging Predictive of Cervical Spondylotic Myelopathy Symptoms

Benjamin Hopkins, BS; Kenneth Weber, PhD; Michael Cloney, MD; Monica Paliwal; Todd Parish, PhD; Zachary A Smith, MD

Introduction: No standard MRI criteria exist for the characterization of cervical spinal injury in patients with Cervical Spondylotic Myelopathy (CSM).1-3 We hypothesize that patients with CSM will exhibit radiographic changes consistent with volume loss leading to decreased spinal cord volume in descending white matter pathways of the spinal cord. These findings predict poor resultant clinical scores.

Methods: Twelve patients $(8$ male, age $61.4 \pm 1.44)$ with CSM and 14 age-matched controls ( 9 male, age-52 \pm 1.55 ) were enrolled prospectively and underwent 3 Tesla MR imaging of the cervical spine. Using the high resolution images $(.47 \mathrm{~mm}$ in plane) of the spinal cord, straightening and alignment with a template was performed and specific spinal cord tract volumes were measured using an open source program, The Spinal Cord Tool-box version 3.0.6 Modified Japanese orthopedic association (mJOA) and Nurick disability scores were collected in all patients in a prospective manner, and were examined in relation to descending spinal tract volumes using single-variable, multivariable, and linear regression analysis.

Results: Anterior/posterior spinal cord diameters ( $\mathrm{p}=0.0016)$, eccentricity of the spinal cord $(\mathrm{p}=0.0114)$, ventral reticulospinal tract volume $(p=0.0026)$, rubrospinal tract volume $(p=0.0024)$, lateral reticulospinal tract volume $(\mathrm{p}=0.0406)$ and ventrolateral reticulospinal tract volume $(\mathrm{p}=0.0035)$ were significantly different between CSM patients and controls. Ventral $(R=0.33, p=0.007)$ and ventrolateral $(\mathrm{R}=0.31, \mathrm{p}=0.017)$ reticulospinal tract volume loss linearly was predictive of worsening mJOA. Moreover, on multivariable logistic regression, ventral reticulospinal tract volume was negatively associated with mJOA scores (OR 0.745 $[0.583,0.953], \mathrm{p}=0.019)$ and Nurick scores (OR 0.773 [0.599, 0.997], $\mathrm{p}=0.047$ ).

Conclusion: Ventral and ventrolateral reticulospinal volume loss on high-resolution MR imaging of the cervical spine, calculated using a noverl, open-source software package, is associated with mJOA and Nurick scores. High-resolution MR imaging and The Spinal Cord Tool-box 3.0 may together provide improved diagnostic accuracy in the preoperative evaluation of patients suspected of having CSM, and improve current understanding of the disease by providing tract-specific data.

\section{Outcomes of Surgical Decompression in Patients with Very Severe Degenerative Cervical Myelopathy}

Branko Kopjar, MD; Parker Bohm; Joshua H. Arnold, BS; Michael G. Fehlings, MD, PhD, FRCS(C), FACS; Lindsay Tetreault, Bsc; Paul M. Arnold, MD

Introduction: Although decompressive surgery has been evidenced as a safe and effective approach for patients with myelopathic deficiencies, studies have suggested residual disability following treatment in patients with more severe disease presentation. The aim of this study was to evaluate outcomes of decompressive surgery in patients with very severe Degenerative Cervical Myelopathy (DCM).

Methods: A prospective observational international study. Postoperative outcomes of 60 patients with very severe DCM (modified Japanese Orthopaedic Association Score (mJOA) $=8)$ were compared to outcomes of 188 patients with severe DCM (mJOA 9 11). Post-imputation follow-up rate was $93.1 \%$. Unadjusted and adjusted analyses were performed using two-way repeated measurest of co-variance.

Results: The two cohorts were similar in demographics, length of duration of myelopathy symptoms, source of stenosis and surgical approaches used to decompress the spine. The very severe and severe cohorts differed in preoperative Nurick grades (4.97 vs. 3.91, respectively, $\mathrm{P}<.0001$ ) and Neck Disability Index (NDI) scores (45.20 vs. 56.21 respectively, $\mathrm{P}=.0006$ ). There were no differences in Short Form 36 (SF-36v2) Physical (PCS) and Mental (MCS) Component Summary Scores. Both cohorts improved in mJOA, Nurick, NDI, and SF-36v2 PCS and MCS scores. Despite the substantial postoperative improvements, patients in both cohorts had considerable residual symptoms. Two-thirds of the patients in the very severe cohort had severe $(\mathrm{mJOA}=11)$ or moderate $(\mathrm{mJOA}=14)$ myelopathy symptoms at 24-months follow-up. Longer duration of disease was associated with poorer treatment response.

Conclusion: Decompressive surgery is effective in patients with very severe DCM, however, patients have significant residual symptoms and disability. The very severe subgroup $(\mathrm{mJOA}=8)$ of patients with DCM represents a distinct group of patients and their different clinical trajectory is important for clinicians and patients to recognize. Duration of symptoms negatively affects chances 
for recovery. Whenever possible, patients with DCM should be treated before developing very severe symptomatology.

470 The Discrepancy Between Clinical Measures and Self-Reported Ratings of Health Status After Surgery for Degenerative Cervical Myelopathy: An Introduction to Response Shift

Lindsay Tetreault, Bsc; Mary Zhu; Rachel Howard; Michael G. Fehlings, MD, PhD, FRCS(C), FACS; Jefferson R. Wilson, $\mathrm{MD}, \mathrm{PhD}$

Introduction: Despite objective clinical improvements following surgery for DCM, experience dictates that a proportion of patients remain dissatisfied with their outcome.

Methods: 401 patients were enrolled at 16 global sites. Functional impairment and self-reported rating of health status were recorded baseline and 1-year postoperative. Concordance was defined as achieving a clinically meaningful improvement (MCID) on the mJOA and reporting general health as somewhat better or much better, whereas discordance was defined as achieving a MCID and reporting general health as the same, somewhat worse or much worse. Logistic regression analysis was used to determine important differences between patients with discrepancies between their clinical measures and self-reported ratings and those without.

Results: Based on patient self-reports, 55 patients were somewhat or much worse than 1-year ago, 82 patients were the same and 264 patients were somewhat or much better. Thirty-one percent of patients who reported being somewhat or much worse achieved a MCID on the mJOA. In addition, $56 \%$ of patients who indicated their health status was the same as one year ago exhibited clinically meaningful improvements in functional impairment. Univariate analyses indicated that a patient is more likely to be dissatisfied with their outcomes if he/she exhibited less improvement in mJOA upper extremity scores $(p=0.071)$, was older $(p=0.0073)$, was a smoker $(\mathrm{p}=0.082)$ and achieved lower total mJOA scores at 1 -year $(\mathrm{p}=0.087)$. Following multivariate analysis, the most important factors were age and improvement in mJOA upper extremity scores.

Conclusion: A response shift in functional outcomes was detected in surgical DCM patients. Older patients, as well as those with smaller improvements in postoperative upper extremity scores, tend to report worsened or unchanged general health status, despite experiencing improvements in overall function.

471 Increase in Spinal Canal Area Following Open-door and Double-door Laminoplasty According to Pre-operative Lamina Angle: Comparative Simulation Study

Jong-myung Jung, MD; Anthony L. Jahng; Seung-Jae Hyun, MD; Ki-Jeong Kim, MD; Tae-Ahn Jahng, MD.Ph.D.; Hyun-Jib Kim

Introduction: Open-door and double-door laminoplasty (ODL and DDL, respectively) have been widely used for the treatment of cervical spondylotic myelopathy or ossification of posterior longitudinal ligament. There were several studies comparing the clinical and radiologic results of ODL and DDL. However, the comparison of increase in spinal canal area (SCA) between the two techniques according to pre-operative lamina angle (PLA) has not been investigated. This study was designed to clarify the relationship between laminoplasty opening angle (LOA) or laminoplasty opening size (LOS) and increase in SCA following ODL and DDL according to PLA using simulation model.

Methods: The validation of the simulation model was analyzed by comparing the clinical data and simulation results of the
64 patients who had undergone C3-C6 laminoplasty (43 patients with ODL and 21 patients with DDL). Laminoplasty (ODL and DDL) with an LOA of 40, 42 and $44^{\circ}$ and LOS of 8, 10, 12 and 14 $\mathrm{mm}$ were simulated to determine the increase in SCA according to PLA (from 28 to $32^{\circ}$ ).

Results: A comparison of the clinical data and simulation results showed no significant difference $(p>0.05)$ and a very high degree of correlation $(\mathrm{p}<0.001)$. These findings support the validity of the simulation model. After ODL, the increase in SCA is constant even with increasing PLA under same LOA or LOS. After DDL, if the PLA increases under same LOA, the increase in SCA increases. However, if the PLA increases under same LOS, the increase in SCA decreases. Under same LOA, the increase in SCA after ODL was significantly greater than the increase in SCA after DDL $(p<0.05)$. Under same LOS, however, the increase of SCA after ODL and DDL showed no significant difference ( $p>$ $0.05)$.

Conclusion: The increase in SCA after ODL and DDL can be predicted by the simulation model. Considering that the instruments used for laminoplasty determine the LOS rather than the LOA, there is no significant difference between the two techniques. It is necessary to understand the advantages and disadvantages of each surgical method and make decisions.

472 Radiographic and CT Evaluation of Recombinant Human Bone Morphogenetic Protein-2-Assisted Anterior Cervical Discectomy and Fusion

\section{Luke Weisbrod; Paul M. Arnold, MD; John Leever, MD}

Introduction: Spinal fusion is often performed to restore spinal stability following surgery for spondylolisthesis, degenerative disc disease (DDD), trauma or infection. [1,2] In the specific setting of cervical radiculopathy due to DDD, anterior cervical discectomy and fusion $(\mathrm{ACDF})$ is a standard-of-care treatment for patients who do not respond to conservative therapy. [3-5] Grafting in ACDF has traditionally been autograft, preferably iliac crest autograft. [6,7] Iliac crest autografts remain the most common site for obtaining autogenous bone grafts. [6] Providing an excellent source of both cortical and cancellous bone, iliac crest autografts are efficacious with respect to fusion. [6] However, grafting bone from the iliac crest is associated with significant morbidity, such as arterial or ureteral injury, herniation, chronic pain, nerve injury, infection, fracture and pelvic instability. [6] This has led to increased exploration for, and use of, alternatives to bone grafts such as synthetic grafts, demineralized bone, ceramics, calcium phosphatases and bone morphogenetic proteins (BMPs). [8-24]

Methods: This study included 26 patients who underwent single-level ACDF with rhBMP-2. All data used for this study was collected from a prior FDA Investigational Device Exemption (IDE) study.

Results: A polyetheretherketone (PEEK) cage was used as an interbody disc spacer in all 26 patients. Patients were evaluated between two and six weeks after surgery and subsequently at 3,6,12, and 24 months postoperative. All patients underwent plain radiography at every follow-up visit, and CT evaluation was performed at 3, 6, 12, and 24 months as part of the study protocol. Earliest fusion was observed at three months in $38 \%$ of patients. Likely fusion was observed in all patients by 12 months postoperative.

Conclusion: rhBMP-2 leads to both successful interbody fusion and an enhanced fusion rate with unique imaging characteristics. Additional characteristics of rhBMP-2 observed in $100 \%$ of patients included pre-vertebral soft-tissue swelling and early endplate resorption. Other common features included PEEK cage migration, heterotopic bone formation, and cage subsidence. 
473 The Effect of C2-C3 Disc Angle on Postoperative Adverse Events in Cervical Spondylotic Myelopathy

Bryan S Lee, MD; Kevin M Walsh, MD; Daniel Lubelski, MD; Emily Hu, BA; Richard C Lee, BA; Maheen Nadeem; Joseph E Tanenbaum, BA; Iain H. Kalfas, MD; Richard P. Schlenk, MD; Thomas E. Mroz, MD; Michael P. Steinmetz, MD; Edward C. Benzel, MD

Introduction: Complete radiographic evaluation is essential in the surgical treatment of cervical spondylotic myelopathy (CSM). Prior studies correlated cervical sagittal imbalance and kyphosis with disability and worse health-related quality of life (HR-QOL). However, little is known about the elevated angle at the $\mathrm{C} 2-\mathrm{C} 3$ disc space (C2-C3 angle) and its correlation with post-operative outcomes. The present study is the first to consider $\mathrm{C} 2-\mathrm{C} 3$ angle as an additional radiographic predictor of clinical outcomes, and we propose the routine evaluation of $\mathrm{C} 2-\mathrm{C} 3$ angle to help optimize surgical outcomes.

Methods: Retrospective chart review was performed to identify CSM patients who underwent surgeries from 2009 to 2017 , and to obtain their demographics, rates of reoperation from instrumentation failure, and HR-QOL. Cervical sagittal alignment variables were measured using the pre- and post-op radiographs. Univariate logistic regressions were used to explore the association between dependent and independent variables, and a multivariable logistic regression model was created using stepwise variable selection as reference by including all independent variables. Nonparametric methods were used to estimate the areas under the receiver operating characteristic (ROC) curves.

Results: We identified 164 patients who had complete pre-and post-operative radiographic and outcomes data. The overall rate of instrumentation failure requiring reoperation was $15.2 \%(25 / 164)$. Only the post-operative C2-C3 angle, sagittal vertical axis, and $\mathrm{T} 1$ tilt were found to be significantly associated with the odds of instrumentation failure. The ROC analysis demonstrated that C2-C3 angle was the most accurate of the models.

Conclusion: In our retrospective analysis of post-operative outcomes of patients with CSM, significant association was found between the $\mathrm{C} 2-\mathrm{C} 3$ angle and post-operative clinical outcomes. We propose that $\mathrm{C} 2-\mathrm{C} 3$ angle be used as an additional parameter of cervical sagittal alignment and predictor for the operative outcomes. C2-C3 angle may also be useful intra-operatively, to serve as a surrogate measure to the global cervical balance.

474 Fatty Infiltration of the Cervical Multifidus Musculature is Associated with Clinical Disability in Cervical Spondylotic Myelopathy: A Prospective, Case-control Series

Michael Brendan Cloney, MD, MPH; Andrew C Smith, DPT, PhD; Taylor Coffey, BS; Monica Paliwal; Yasin Dhaher, PhD; Todd B. Parrish, PhD; James M. Elliott; Zachary Adam Smith, MD

Introduction: Cervical spondylotic myelopathy (CSM) is among the most common spinal cord disorders of the elderly. Muscle fat infiltration (MFI), the pathological infiltration of fatty tissue into muscle, is known to contribute to pain and disability following in a variety of neurologic and spinal pathologies [1-7], but has never been studied in patients with CSM. We examined the relationship between MFI and clinical disability from CSM.

Methods: We prospectively enrolled nine CSM patients and five aged-matched controls to undergo MRI imaging of the cervical spine with MFI. A 3 Tesla, 3 dimensional dual-echo gradient echo acquisition was performed to collect fat and water data for the neck multifidi muscles (C3 C7) to calculate MFI. A blinded investigator manually traced regions of interest for each of the bilateral multifidii muscles from $\mathrm{C} 3$ to $\mathrm{C} 7$ on fat-water MRI images to calculate MFI percentages. Nurick scores and modified Japanese Orthopedic Association scores were collected for all patients.

Results: CSM patients and controls were equivalent with respect to age, height, weight, gender, race, smoking status, and employment status. CSM patients and controls differed with respect to both mJOA scores $(14.6 \pm 0.6,18.0 \pm 0.0, \mathrm{p}=0.0017)$ and Nurick scores $(1.9 \pm 0.3$ v. $0.0 \pm 0.0, \mathrm{p}=0.0008)$. MFI was higher in patients with CSM than in controls $(31.7 \%$ v. $24.6 \%$, respectively, $\mathrm{p}=0.0178)$. MJOA scores correlated linearly with MFI $(\mathrm{R}=0.542$, $\mathrm{p}=0.0453)$. Higher MFI was associated with increased disability on the Nurick scale $(\mathrm{p}=0.0371)$.

Conclusion: Cervical spondylotic myelopathy is associated with increased MFI of the multifidus muscles, which play a critical role in biomechanical stability of the spine [8]. MFI is correlated with clinical disability, as measured by mJOA and Nurick scores. Spinal injury in CSM may lead to muscle fat infiltration, exacerbating the disability associated with the disease.

475 Vascularized Occipital Bone Graft to Supplement C1-C2 Arthrodesis in Patients with Failed Fusion

Aditya Vedantam, MD; Sungho Lee, MD; Mohin Bhadkamkar, MD; Michael Bohl, MD; U. Kumar Kakarla, MD; Nicholas Theodore, MD; Edward Reece, MD; Alexander E. Ropper, MD

Introduction: Atlantoaxial pseudoarthrosis is a challenging problem, and may result from a combination of local and systemic factors. The use of a local, vascularized bone graft to supplement C1-C2 arthrodesis has not been described for patients with, or at risk for atlantoaxial pseudoarthrosis. We report the first surgical patient who received a vascularized pedicled occipital bone graft for supplementation of arthrodesis.

Methods: We describe the technique for using a vascularized occipital bone graft in a 72-year old lady with failed C1-C2 arthrodesis after a prior fusion. The patient presented with increasing neck pain, and imaging demonstrating pseudoarthrosis at C1-C2 with bilateral $\mathrm{C} 1$ lateral mass screw fractures. A surgical plan was made to perform $\mathrm{C} 1-\mathrm{C} 2$ fixation and fusion with transarticular screws, supplemented with a vascularized occipital bone graft.

Results: At surgery, the prior instrumentation was removed and transarticular $\mathrm{C} 1-\mathrm{C} 2$ screws were placed. A vascularized occipital bone graft based on the rectus capitis posterior major and minor muscles was marked. The paramedian bone graft was harvested from between the superior nuchal line and foramen magnum. The muscular attachments were preserved, and the flap was rotated into the area between $\mathrm{C} 1-\mathrm{C} 2$ after decortication of the $\mathrm{C} 1$ arch and $\mathrm{C} 2$ spinous process. A cross-connector between the rods at $\mathrm{C} 1-\mathrm{C} 2$ interspace was placed to hold the graft in place, and the wound was closed in layers. The patient was fitted with a cervical collar for 6 weeks. Postoperative CT imaging at 3 months showed a healthy graft with early fusion between $\mathrm{C} 1$ and $\mathrm{C} 2$.

Conclusion: We demonstrate the feasibility of using a local, vascularized occipital bone graft to supplement $\mathrm{C} 1-\mathrm{C} 2$ arthrodesis. This graft has several inherent advantages over other common bone grafts, and represents a novel technique to treat patients with, or at risk, for failed $\mathrm{C} 1-\mathrm{C} 2$ arthrodesis.

476 Quality of Life and Surgical Outcomes of Mild Degenerative Cervical Myelopathy

Jetan H. Badhiwala, MD; Christopher D. Witiw, MD; Farshad 
Nassiri, MD; Muhammad Akbar, MD; Alireza Mansouri, MD; Jefferson R. Wilson, MD, PhD; Michael G. Fehlings, MD, PhD, FRCS(C), FACS

Introduction: There is controversy over the optimal treatment strategy for patients with mild degenerative cervical myelopathy (DCM), as reflected in recent clinical guidelines(1). In a prospective cohort of mild DCM patients undergoing surgical decompression, we sought to evaluate the degree of impairment in baseline quality of life as compared to population norms, as well as functional, disability, and quality of life outcomes following surgery.

Methods: We identified patients with mild DCM (mJOA 15 to 17)(2) enrolled in the prospective, multi-center AOSpine CSM$\mathrm{NA}(3)$ or CSM-I(4) trials. Baseline quality of life (SF-36v2) was compared to Canadian population normative data(5) by the standardized mean difference (SMD). Outcomes, including functional status (mJOA, Nurick grade), disability (NDI), and quality of life (SF-36v2), were evaluated at baseline and 6 months, 1 year, and 2 years after surgery. Post-operative complications within 30 days of surgery were monitored.

Results: 193 patients met eligibility criteria. Mean age was 52.4 years. There were 67 females $(34.7 \%)$. Patients had significant impairment in all domains of the SF-36v2 compared to population norms, greatest for Social Functioning (SMD -2.33), Physical Functioning (SMD -2.31), and Mental Health (SMD -2.30). A significant improvement in mean score from baseline to 2-year follow-up was observed for all major outcome measures, including mJOA $(0.87, \mathrm{P}<0.01)$, Nurick grade $(-1.24, \mathrm{P}<0.01)$, NDI $(-12.97, \mathrm{P}<0.01)$, and SF-36v2 PCS $(5.75, \mathrm{P}<0.01)$ and MCS $(6.93, \mathrm{P}<0.01)$. The rate of complication was low.

Conclusion: Even 'mild' DCM is associated with significant impairment in quality of life. Surgery results in significant gains in functional status, level of disability, and quality of life. It is perhaps time we recalibrate the way we think of the goals of surgery in this population to extend beyond preventing neurological deterioration, to improving function and quality of life, as stability may not be a "good" or acceptable outcome to patients.

477 Is Fixation Necessary After Laminectomy in Ossification of the Posterior Longitudinal Ligament? A Comparative Study Between Laminectomy Only and Laminectomy and Lateral Mass Screw

\section{Gwangsoo Lee}

Introduction: The effect of laminectomy in OPLL patients is known, but there is concern about subsequent complications such as the occurrence of kyphotic changes. Some of the papers suggested that occlusion foci of OPLL would stabilize and reduce postoperative kyphotic change. In this study, we would like to see the results of this opinion by comparing laminectomy only group with laminectomy and LMS group in OPLL patients.

Methods: One hundred forty nine patients who underwent cervical laminectomy with or without lateral mass screw fixation for OPLL from 2011 to 2016 were enrolled. The clinical outcomes were assessed using neck disability index, visual analogue scale, and orthopedic association scoring systems. The radiologic evaluation measured sagittal alignment on plain cervical lateral radiographs.

Results: There was no difference between the two groups in the perioperative clinical outcome. Changes in kyphotic angle occurred in 3 out of 32 patients in the laminectomy only group and 11 out of 117 patients in the laminectomy and LMS group. There was a decrease in cervical lordosis after surgery, but the difference between the two groups was not statistically significant.

Conclusion: There was no statistically significant difference between two groups in OPLL patients. If stabilization is achieved due to OPLL, we think it is possible to obtain favorable clinical outcome without LMS after laminectomy. We will then conduct a study to clarify the criteria for the separation of fixation and nonfixation in OPLL patients.

478 The Utility of Intra-operative Neurophysiological Monitoring for Routine Anterior Cervical Discectomy and Fusion Surgery

Jetan H. Badhiwala, MD; Farshad Nassiri, MD; Christopher D. Witiw, MD; Alireza Mansouri, MD; Saleh A. Almenawer, MD; Leodante da Costa; Michael G. Fehlings, MD, PhD, FRCS(C), FACS; Jefferson R. Wilson, MD, PhD

Introduction: The utility of intra-operative neurophysiological monitoring (IONM) for ACDF surgery is debated $(1,2)$. SSEPs may be insufficiently sensitive and specific(3-7). Proponents of IONM advocate for multimodal monitoring, including tceMEPs $(4,8)$. Still, the benefit to outcomes is unclear(2). There are few head-to-head comparisons of outcomes with and without IONM(7). Using an administrative health database, we examined neurological complications of ACDF surgery with versus without IONM.

Methods: Hospitalizations with a primary procedure code for ACDF (ICD-9-CM 81.02) were extracted from the 2009-2013 U.S. National Inpatient Sample. We identified operations that used IONM (ICD-9-CM 00.94). Data on patient- and treatment-related covariates were extracted. The primary outcome was post-operative neurological complication (ICD-9-CM 997.0, 997.00, 997.01, $997.02,997.09)$. We performed logistic regression for the primary outcome with IONM and relevant covariates as independent variables. We also performed a propensity-matched analysis evaluating post-operative complication and hospital charges with IONM use as the treatment indicator. We evaluated the impact of IONM on neurological complication in relevant subgroups of patients.

Results: 141,007 ACDF operations were identified, with IONM used in 9,540 (6.8\%). Neurological complication occurred in $291(0.21 \%)$ cases. IONM did not impact the rate of complication on univariate $(\mathrm{p}=0.39)$ or multivariate $(\mathrm{p}=0.45)$ regression. The propensity-matched cohort consisted of 9,380 patients who underwent ACDF with, and 9,380 without, IONM. The two groups did not differ with respect to important covariates. Neurological complication was comparable between IONM and non-IONM groups $(0.17 \%$ vs. $0.22 \%$; $p=0.41)$. Use of IONM led to an additional $\$ 6,843(\mathrm{p}<0.01)$ in hospital charges. We did not identify a subgroup of patients defined by age, sex, surgical indication, number of levels fused, Charlson Comorbidity Index, or admission type where IONM was associated with a significant difference in neurological complication.

Conclusion: The routine use of IONM for ACDF appears to provide no benefit to patient safety at additional cost.

480 Magnetic Resonance Imaging Findings Predict Overall Survival and Local Recurrence in Patients Undergoing En Bloc Resection of Sacral Chordomas

Scott L. Zuckerman, MD; Behrang Amini, MD, PhD; Sun-Ho Lee, MD; Claudio Esteves Tatsui, MD; Laurence D. Rhines, MD

Introduction: Few studies have assessed the importance of preoperative magnetic resonance imaging (MRI) scan findings in patients undergoing en bloc resection of sacral chordomas. The current objectives were to: 1) report imaging characteristics of sacral chordomas; 2) describe the patterns of local and distant recurrence, and 3) determine imaging predictors of overall survival (OS) and local recurrence (LR). 
Methods: This retrospective case series was obtained from a prospectively maintained spine database from 1995-2016. All patients underwent en bloc resection of sacral chordomas. Detailed demographic, outcome, and MRI scan data were collected. A survival analysis was completed with multivariable cox regression to assess the impact of MRI findings on OS and LR.

Results: A total of 41 patients underwent en bloc resection of their sacral chordoma. Tumor characteristics were mostly lobulated $(93 \%)$ with a soft tissue tail $(54 \%)$ in over half. The following areas had tumor invasion: SI joint (15\%), ilium $(5 \%)$, piriformis $(61 \%)$, gluteus $(46 \%)$, subcutaneous fat $(32 \%)$ and epidural venous plexus (22\%). Only subcutaneous fat extension was an independent predictor of decreased OS after multivariable analysis (HR 5.30, 95\% CI 1.47-19.19, $\mathrm{p}=0.011$ ). The following factors were significant predictors of LR after log-rank testing: above the L5/S1 disc space ( $\mathrm{p}=0.004)$, SI joint invasion $(\mathrm{p}=0.036)$, and piriformis extension $(\mathrm{p}=0.022)$, but were not significance after multivariable analysis.

Conclusion: The presence subcutaneous fat extension was an independent predictor of decreased OS. Other MRI findings with potential for future evaluation include size, presence of soft tissue tail, extension above L5/S1, and SI joint and piriformis invasion.

\section{Brachytherapy in Spinal Tumors: A Systematic Review}

Scott L. Zuckerman, MD; Mark H. Bilsky, MD; Yoshiya Josh Yamada, MD, FRCP; Ilya Laufer, MD

Introduction: While external beam radiation is commonly used to treat spinal tumors, intraoperative brachytherapy is an alternative option for radiation delivery. We review the use of brachytherapy in spine tumor surgery with regards to: 1) presentation, 2) indications, 3) delivery, and 4) outcomes.

Methods: Pubmed and Embase databases were systematically queried for literature reporting the use of brachytherapy for the treatment of spinal tumors. Data extraction for each study was performed. Standard descriptive statistics were calculated.

Results: Of 204 studies, 15 met inclusion criteria. PRESENTATION. A total of 379 patients underwent spinal brachytherapy, with mean age of 57.8 years (SD 7.1, range 6-89). Metastatic disease was treated in 329 patients $(87 \%)$ and primary tumors were treated in 50 patients $(13 \%)$. INDICATIONS. Brachytherapy indications included: previous treatment (6), intractable pain (6), could not tolerate open surgery (4) or chemotherapy/ radiation (1), or unresectable tumor (1). DELIVERY. Commonly used elements were: I125 (9), Ir192 (4), P32 (2), Y90 (1), Sm153 (1). Seeds were used most often (10) followed by plaque/implant (5). Open surgery was performed in 8 studies and percutaneous placement in 6 (one study reported both), with cement in 7 studies and hardware in 6. OUTCOMES. Pain was measured in 10 studies and performance/neurologic status measured in 7 . Local recurrence data (range 0-49\%) was reported in 9 studies. All studies that reported pain and performance measures reported a statistically significant improvement.

Conclusion: The use of brachytherapy for the treatment of spinal tumors is limited. Tumor recurrence after radiotherapy serves as the most common brachytherapy indication. Significant improvements in pain, performance, and neurologic status were commonly reported. Brachytherapy serves as a viable treatment option for patients with recurrent tumors or tumors at high risk for recurrence. Delineation of optimal brachytherapy indications and methods requires comparative efficacy analyses in larger patient populations.
482 Spine Metastases Predominantly Localize to the Posterior Aspect of the Vertebral Body

Jason Kessler; Jonah Yousif, BS; Eleanor Smith, BS; Marissa Guo; Kristen Kolberg; Joseph Raynor Linzey BS; Greg Clines, MD, PhD; Alexandra Calinescu, MD, PhD; Spratt Daniel, MD; Brandon W. Smith, MD, MSCR; Paul Park, MD; Nicholas J. Szerlip, MD, FACS

Introduction: Spine metastases pose significant morbidity for patients. For many histologies, the spine is a frequent site for bone metastases. This predilection is not fully understood and there are conflicting reports regarding the distribution within the vertebral body itself. Knowing this distribution will give us clues as to the underlying biologic reason for this increased incidence in the spine and lead to a better understanding of tumor dispersion and growth.

Methods: We retrospectively examined MRIs of patients undergoing radiation to the spine from 2015 to 2017 for spinal metastases. The anatomical distribution of lesions was categorized. Lesions were sorted along the sagittal plane into five groups: Anterior only (A), Anterior + Middle (AM), Middle only (M), Posterior + Middle (PM), and Posterior only (P). Lesions that covered all groups were discarded. Chi-square and post-hoc analyses were used for statistical analysis.

Results: Eighty-nine patients with a total of 300 metastatic lesions were examined. Two hundred and three lesions were used for analysis. Sixty-five percent of all lesions were found in $\mathrm{P}$ and PM aspects of the vertebral body $(\mathrm{p}<0.0001)$. This localization was significant regardless of histology: Lung $(67 \%, \mathrm{p}<0.0001)$, Kidney $(66 \%, \mathrm{p}<0.0001)$, Sarcoma (67\%, $\mathrm{p}<0.0001)$, Prostate $(63 \%, \mathrm{p}=0.01)$, and Breast $(63 \%, \mathrm{p}=0.01)$. This was consistent across thoracic $(n=96)$ and lumbar $(n=63)$ regions $(72 \%$ and $64 \%$ respectively, $\mathrm{p}<0.0001)$. The cervical region $(\mathrm{n}=27)$ did not show a significant posterior distribution.

Conclusion: Metastatic lesions of the thoracolumbar spine have a greater propensity to localize to the posterior aspect of the vertebral body. These data support the hypothesis that there may be differences within the vertebral body leading to differential tumor dispersion and growth. In the future we will examine the local micro-environmental changes across the vertebral body that can contribute to this propensity for posterior permissive growth of metastases.

483 Adverse Events Profile in En Bloc Resection for Metastasis and Surgery for Primary Bone Tumor

Shreya Srinivas, FRCS; Raphaele Charest-Morin; Charles Fisher, MD; Heidi Marie Britton; Tamir T. Ailon, MD, MPH; Michael C. Boyd, MD; Marcel Dvorak, MD; Brian K Kwon, MD, PhD, FRCSC; Scott J. Paquette, MD, FRCS(C); John Street; Nicolas Dea, MD, MSc, FRCSC

Introduction: En bloc resection is uncommonly performed in the spine and this study aims to determine adverse event (AE) profile in those undergoing en bloc resection for spinal metastases or surgery for primary bone tumor of spine.

Methods: This is a prospective cohort study in a single quaternary care referral center of consecutive patients (Jan 2009 to July 2017) who underwent en bloc resection for spinal metastases or surgery for primary bone tumor of the spine. AE were collected on a standardized form (Spine AdVerse Events Severity System, version 2 [SAVES V2] forms) at weekly-dedicated morbidity and mortality rounds. Data collected included patient demographics, primary tumor histology, neurological status, surgical intervention details, marginal status, Enneking appropriateness and all AEs (peri \& post operative) 
Results: We treated 112 patients (64 female, 40 male, median age 51 years, 116 procedures) with primary bone tumor (96) and metastatic lesions (16). In the primary tumors, surgical resection was Enneking appropriate (EA) resection in $73 \%$ and Enneking inappropriate (EI) in $27 \%$ of procedures. At least $1 \mathrm{AE}$ occurred in $70.6 \%$ of procedures and there was 1 death seen. Intraoperative AEs occurred in $27.7 \%$ due to massive blood loss $(23 \%)$ dural tear $(19.5 \%)$, visceral or neuro vascular injury $(20.7 \%)$.Post operative AE incidence was $65.5 \%$ and due to systemic infection (39.5\%); cardiac event (35.5\%); delirium (23.6\%) and thromboembolic events $(10.5 \%)$. Overall implant related complications was low (12\%). AE with EI resection (75\%) was higher than if a EA resection was performed $(66 \%)$ but similar irrespective of tumour pathology (69\% in metastatic lesions and $71 \%$ for primary bone tumor). Wound related complications $(22.4 \%)$ was higher in lesions around occiput or sacrum (64\%) than mobile spine (10\%).

Conclusion: Enbloc resection for metastatic lesions and surgery for primary bone tumors is associated with high incidence of $\mathrm{AE}$.

484 Steroid Use Associated with Increased Odds of 30-Day Mortality in Surgical Patients with Spinal Tumors in the Setting of Disseminated Cancer: A NSQIP Database Analysis

Jonathan Hobbs, MD; Prateek Kumar; Edwin Ramos, MD; Ankit Indravadan Mehta, MD

Introduction: Metastatic spinal cord compression (MSCC) develops in 5-10\% of all cancer patients.[5] Steroid administration is part of a standard treatment regimen, along with radiation or surgical interventions.[6] The appropriate dose, duration, efficacy and risks of steroid administration remain controversial.[1-4,7-9] Our study uses big data systems to analyze the risk of perioperative steroid use in a specific surgical cohort.

Methods: We conducted a retrospective, cohort study, evaluating surgical spine tumor cases from the American College of Surgeons (ACS) National Surgical Quality Improvement Program (NSQIP) database. CPT codes designated for surgical intervention on spine tumors identified cases for inclusion. Steroid use is defined as chronic (>10 days) use of steroids within 30 days prior to or at the time of consideration for operation. Steroid boluses and short durations or tapers ( $<10$ days) are not included. The association between steroid administration and 30-day mortality was analyzed using chi-squared analysis and multivariate logistical regression. Additionally, chi-squared analysis and univariate logistic regression provided an assessment for confounding factors that might influence this association. Statistical software constructed a final logistical model to assess 30-day mortality and significant variables in the NSQIP dataset.

Results: In this cohort of 1262 surgical spinal tumor patients, $132(10.4 \%)$ received pre-operative steroids. The incidence of 30-Day mortality was significantly greater in patients on steroids (chi-squared $\mathrm{p}<0.001$ ). Univariate logistical regression identified statistically significant variables associated with mortality. Forward and reverse stepwise model selection utilizing a significance level of $\mathrm{p}<0.05$ to build a final model with these variables. In this multivariate model, steroid use was associated with a 2.57 times greater odds of death within 30 days.

Conclusion: Steroid use is associated with a dramatically increased odds of 30-day mortality in surgical spine tumor patients with disseminated disease. This study may support localized steroid therapy to reduce detrimental systemic effects
485 Intrathoracic Schwannomas and it's Surgical Considerations and Approaches.

Line G. Jacques, MD, MSc, FRCS(C), DABNS

Introduction: The most common peripheral nerve tumors are often benign, and consists of schwannomas, or neurofibromas with or without NF1. Most often the localization of these tumors will be in the upper or lower extremities. The intrathoracic localization is usually incidental and requires in some instances, specific surgical approaches. The optimal approach depends on the location of the tumor (posterior-middle-anterior compartments of the thoracic inlet), the depth and the extent of the tumor.

Methods: This is a retrospective study looking at 12 patients with intrathoracic schwannomas. Their clinical and radiological presentation will be reviewed; an intercostal schwannoma, phrenic nerve schwannoma in the pulmonary aortic window, T1-T2, T1-T4, and T4-T6, and their respective intraoperative surgical approaches.

Results: Twelve patients with peripheral nerve sheath tumor in the lower plexus with intra-thoracic component are being presented with the preferred surgical approach for each case. The location of the mass, along with the surgeon's experience will determine the best surgical approach to achieve a complete resection.

Conclusion: Intrathoracic tumors tend to present incidentally. When they are symptomatic or show significant growth, a surgical intervention may be necessary to provide a good outcome, especially if the pathology demonstrates the schwannoma's pathognomonic features.

486 Retrospective Analysis of Multimodal Treatment of Spine Tumors in Patients with Neurofibromatosis Type 2

Richard L Price, MD, PhD; Travis BS CreveCoeur; Brian Chu; Michael R. Chicoine; Wilson Zachary Ray, MD

Introduction: Neurofibromatosis type II (NF2) is an autosomal dominant disease that results in multiple tumors of the brain and spine. Many patients have a high burden of disease making optimal disease management challenging. We report our experience treating spine tumors of NF2 patients.

Methods: A retrospective review of 61 patients with NF2 identified via ICD-9 code was performed using our brain tumor registry at Washington University. Data collection included demographics, age at diagnosis, imaging data of spinal disease, physical exam, surgical treatments, chemotherapy treatments, and outcomes. Statistical analyses were performed with SAS 9.4.

Results: Of the 61 NF2 patients treated at our institution, 54 patients had MRI evidence of tumors involving the spine. Patients had an average of 6.5 (range: 1-innumerable) tumors. Patients most commonly had lesions in the cervical spine $(81.3 \%)$, followed by lumbar $(81.3 \%)$ and thoracic $(72.9 \%)$. In the majority of patients $(60.4 \%)$, tumors were widespread involving all regions of the spine. Despite high tumor burden, most patients were managed conservatively, with only $42.6 \%$ (23/54) requiring surgery. Cervical spine tumors were the most common site requiring surgery $(42 \%, 13 / 31)$. Tumor burden did not correlate with the need for surgery. Pain and ataxia were the most common indications for surgery. Schwannomas were the most common types of tumors resected $(62.9 \%, 17 / 27)$. Additionally, 16 patients have been treated with Bevacuzimab for tumor management. None of the patients treated with Bevacuzimab experienced decrease in tumor size and only $12.5 \%$ experienced a decrease in symptoms. Several patients still underwent surgery despite Bevacuzimab.

Conclusion: Spine tumor burden of NF2 patients does not 
correlate with needing surgery. Cervical spine tumors are the most common tumors needing resection for ataxia or pain. Our experience shows that Bevacuzimab administration does not decrease size of NF2 spine tumors. More data is necessary to validate use of Bevacuzimab for spine tumors.

\section{Adult Intramedullary Astrocytomas in Adult Patients}

Owoicho Adogwa, M.D., M.P.H; Syed Ibad Khalid; Ryan Kelly, BS; Victoria D Vuong; Ankit Indravadan Mehta, MD; Carlos Antonio Bagley, MD; Joseph S. Cheng, MD, MS

Introduction: In adults, spinal astrocytomas constitute only a small fraction central nervous system tumors. Due to their rarity and poor prognosis, large population-based studies are needed to assess the epidemiology and survival risk factors associated with these tumors in the hope of improving outcome. The authors undertook this retrospective study to explore factors that may influence survival in adult patients with spinal astrocytomas.

Methods: Utilizing the Surveillance, Epidemiology, and End Results (SEER) database, a prospective cancer registry, the authors retrospectively assessed survival in histologically confirmed spinal astrocytomas in patients 18 years of age and older. Survival was described with Kaplan-Meyer curves, and a multivariate regression analysis was used to assess the association of several variables with survival while controlling for confounding variables.

Results: Patient age at diagnosis (hazard ratio [HR] 1.52, 95\% CI $1.17-1.99, \mathrm{p}<0.001$ ), tumor grades of IV (HR 8.85, 95\% CI $2.83-27.69, \mathrm{p}<0.001$ ), and the utilization of biopsy (HR 6.26, CI $1.41-27.69, \mathrm{p}=0.016$ ) or partial resection (HR 5.80, 95\% CI 1.20 $28.03, \mathrm{p}=0.029$ ) without the performance of a complete resection were each associated with decreased survival.

Conclusion: High-grade ependymomas occurring in the spine have a much worse prognosis. Early diagnosis and surgery appear to be associated with improved survival and outcomes, while radiation therapy has an unclear role. Study

488 Pediatric Spinal Ependymomas: An Epidemiological

Owoicho Adogwa, M.D., M.P.H; Syed Ibad Khalid; Ryan Kelly, BS; Victoria D Vuong; Josha Woodward, BS; Shaheen Ahmed, PhD; Ryan Khanna, MD; Carlos Antonio Bagley, MD; Joseph S. Cheng, MD, MS; Sanjit Shah; Ankit Indravadan Mehta, MD

Introduction: Pediatric intramedullary spinal cord ependymomas represent a rare central nervous system neoplasm with little available data regarding incidence and outcomes. To this end, large population-based studies are needed to assess the epidemiology and survival risk factors associated with these tumors in the hope of better understanding these tumors as well as improving outcomes. The authors undertook this retrospective study to explore factors that may influence survival in pediatric patients with intramedullary spinal cord ependymomas.

Methods: Utilizing the Surveillance, Epidemiology, and End Results (SEER) database, a prospective cancer registry, the authors retrospectively assessed survival in histologically confirmed spinal ependymomas in patients 17 years of age and younger. Survival was described with Kaplan-Meier curves, and a multivariate regression analysis was used to assess the association of several variables with survival while controlling for confounding variables.

Results: Invasive tumor extension $(\mathrm{p}<0.001)$ was associated with decreased survival while gross total resection $(\mathrm{p}=0.028)$ correlated with better rates of survival. Age, gender, tumor size, tumor extension, the utilization and sequence of radiation therapy, or utilization of chemotherapy were not found to have a statistically significant association with survival outcomes

Conclusion: Invasive ependymomas occurring in the spine have a much worse prognosis, while higher tumor grades do not clearly demonstrate worse rates of survival. Early diagnosis and surgery appear to be associated with improved survival and outcomes, while radiation therapy and chemotherapy has an unclear role.

489 Surgical Outcomes and 30-Day Complication Rates Associated with Primary Spinal Intramedullary Ependymoma Resection

Aladine A. Elsamadicy, BE; Owoicho Adogwa, M.D., M.P.H; Sohrab Vatsia; Victoria D Vuong; Daniel Lilly, B.S.; Shyam Desai; Joseph S. Cheng, MD, MS; Carlos Antonio Bagley, MD; Isaac O. Karikari, MD

Introduction: Intramedullary spinal cord tumors (IMSCT) are relatively uncommon lesions, with ependymomas being the most common of them. While previous studies have identified complication rates associated with extrmedullary spinal cord tumors, there is a paucity of data identifying post-operative and 30-day complication rates associated with IMSCT- specifically ependymomas.

Methods: The medical records of 72 adult (=18 years old) cases of elective spine surgery for primary resection of IMSCT at a major academic institution from 2005 to 2015 were reviewed. Patient demographics, comorbidities, tumor size and location were collected for each patient. Tumor pathology was analyzed and diagnosed by a board-certified pathologist. The primary outcomes were intra- and 30-day post-operative complication and readmission rates.

Results: Of the 72 IMSCT resection cases, 24 (33.3\%) cases were identified as ependymomas by pathology. The majority of the cohort were female $(54.2 \%)$ and White $(87 \%)$, with a mean age of $45.8 \pm 14.2$ years old. The mean tumor size was $4.2 \pm 4.7 \mathrm{~cm} 2$ with cervical $(62.5 \%)$ and thoracic $(33.3 \%)$ as the most common locations. The majority of patients had an open surgery $(83.3 \%)$, with a median number of laminectomy levels of 3 [2.25-4] and mean operative time of $290.7 \pm 112.0$ mins. There was no spinal cord or nerve root injuries. Post-operatively, $91.7 \%$ of patients were transferred to the intensive care unit. The most common post-operative complications were sensory deficits $(8.3 \%)$, new weakness $(4.2 \%)$, and hypertension (4.2\%). The mean length of stay was $4.5 \pm 1.7$ days, with the majority of patients discharged home $(95.8 \%)$. The 30-day readmission rate was $12.5 \%$, with the most common 30 -day complications being uncontrolled pain $(12.5 \%)$, urinary tract infections $(8.3 \%)$, and wound bleeding $(4.2 \%)$.

Conclusion: Our study suggests there is a low complication rate associated with elective surgical resection of intramedullary ependymomas. However, there is a relatively high 30-day readmission rate with uncontrolled pain being the most common 30-day complication.

490 Management of Sacral and Vertebral Chordomas: Survival Analysis and Comparison of Treatment Modalities

Mohamad Bydon, MD; Yagiz Ugur Yolcu; Waseem Wahood, MS; Panagiotis Kerezoudis; Mohammed Ali Alvi, MD; Sandy Goncalves; Mohamed Elminawy, MBBCh; Mohammed Adeeb Sebai; Redab Alkhataybeh; Anshit Goyal, MBBS

Introduction: Chordomas are slow-growing but invasive tumors that arise from remnants of notochord. Standard of care 
consists of surgical resection and radiotherapy when complete resection isn't possible. Current literature has reached equivocal results with regards to the effect of adding radiotherapy on patient survival.

Methods: The National Cancer Database (NCDB) was queried for patients diagnosed with vertebral chordoma between 2004 and 2010. Overall, survival comprised of the primary outcome of interest and was assessed using Kaplan-Meier plots. Multivariable proportional hazards Cox regression was subsequently performed after adjusting for an array of patient demographics, facility type and tumor characteristics.

Results: A total of 500 chordomas (292 in sacrum, 208 in vertebral column) were identified. Mean age (SD) for sacral and vertebral chordoma patients was $60.6( \pm 14.2)$ and $58.0( \pm 15.8)$, respectively $(\mathrm{p}=0.026)$. Median follow-up periods (interquartile range) for the two groups were 65.7 (41.7) and 62.3 (37.5) months. Three hundred thirty-three patients $(66.6 \%)$ were treated with surgery alone, and one hundred and sixty-seven (33.3\%) received radiotherapy in addition to surgery. Median overall survival was 10.2 and 10.4 years for sacral and vertebral chordomas, respectively $(\mathrm{p}=0.20)$. Median survival was 8.6 and 10.4 years for patients receiving adjuvant radiotherapy and surgery alone, respectively. On multivariable analysis, adjuvant radiotherapy was not associated with decreased risk of death compared to surgery alone (HR:0.88, 95\% C.I:0.5 to $1.6, \mathrm{p}=0.69$ ). Similarly, no difference was found between sacral and vertebral chordomas (HR: 1.45, 95\% CI: 0.79 to $2.7, \mathrm{p}=0.23$ ).

Conclusion: Based on data from a large national cancer registry, we found that there is not enough evidence to suggest that adjuvant radiotherapy offers survival benefit in patients with newly diagnosed vertebral chordomas. Given the non-negligible complications associated with pelvic and vertebral radiation, balance of benefits and risks needs to be taken into consideration during preoperative tailoring of decision making.

491 Implication of Biomarker Mutations for Predicting Survival in Patients with Metastatic Lung Cancer to the Spine

Bryan D. Choi, MD; Ganesh Shankar, MD, PhD; Ahilan Sivaganesan, MD; Vijay Yanamadala, MD, MBA; Laura Van Beaver; Kevin, MD Oh; John H. Shin, MD

Introduction: Spinal metastases are a major cause of pain and neurologic impairment in patients with cancer. Molecular profiling strategies to characterize lung cancer have identified several biomarkers and gene expression signatures that may lead to more effective prognostication and personalized treatment. The aim of this study was to ascertain whether molecular phenotype in patients with advanced metastatic disease is associated with survival, when the disease has progressed to the spine.

Methods: We queried our institutional database for patients with metastatic lung cancer who underwent treatment for spinal metastases between 2011 and 2017. Reports were reviewed for molecular phenotype. Survival time was the duration between treatment for spinal metastases and death. Kaplan-Meier methods and the log-rank test were used to characterize and compare survival data.

Results: Twenty-six patients met criteria for inclusion in this study. Median survival following surgery was 0.67 years. Median overall survival (OS) following diagnosis was 2.7 years. Genetic mutations in ALK, MET, ROS1, EGFR, and KRAS were chosen for study based on their availability and significance in oncologic disease. When considered together, the presence of these molecular abnormalities in patients with spinal metastases was significantly associated with increased OS (HR $0.38,95 \%$ CI $0.12-1.22$, $\mathrm{P}=0.03$ ).
Conclusion: Molecular phenotyping may provide prognostic insight in patients undergoing surgery for spinal metastases. The results presented here suggest that the presence of a mutation in one of five genes is associated with improved survival. This is the first study to demonstrate a significant association between genetic mutational data and overall survival in this patient population. It also represents the largest published series of patients $(n=26)$ with metastatic lung cancer to the spine for which genetic mutational data are reported. Future models estimating survival for patients with spinal metastases may be enhanced by the incorporation of molecular phenotype criteria.

492 Perineural Spread of Prostate Cancer to the Lumbosacral Plexus

Jonathan Jay Stone, MD; Ann T. Packard; Stephen M. Broski; Mark A. Nathan; Benjamin M. Howe; Robert J. Spinner, MD

Introduction: Perineural spread of prostate cancer into the lumbosacral plexus and pelvic peripheral nerves is a rare but increasingly recognized entity (1-3). This form of metastasis infiltrates any layer of the nerve sheath and is thought to reach the lumbosacral plexus via retrograde travel through the splanchnic and hypogastric nerves innervating the prostate. The prevalence of perineural spread is likely underappreciated and further imaging based studies are needed to elucidate its true frequency.

Methods: A retrospective review was performed utilizing an institutional radiology database. Dictations from prostate cancer patients undergoing PET/CT were queried for the following search terms: perineural, sciatic, femoral, obturator, or lumbosacral plexus. PET and MR imaging from identified patients were blindly reviewed for peripheral nerve involvement by two nuclear medicine and two musculoskeletal radiologists.

Results: A total of 16 patients with evidence of perineural metastasis on C-11 choline and/or F-18 FDG PET/CT were identified (Figure 1). Fourteen of these patients also underwent MRI, which confirmed neural abnormality, including enlargement, increased T2 signal, and post-gadolinium enhancement (Figure 2). Findings were frequently multifocal and included S1-S4 nerve roots, lumbosacral trunk, obturator nerve, or intrapelvic and extrapelvic sciatic nerve. Denervation changes were also found involving the piriformis, gluteal, quadratus femoris, hamstring, and obturator internus muscles. Five cases were proven by nerve biopsy. There was very good concordance between histopathological diagnosis and imaging modalities with high confidence among radiologists.

Conclusion: Perineural spread of prostate cancer appears to be more common than previously thought. While MRI remains the gold standard modality for imaging perineural metastatic disease, many of these patients undergo PET/CT to evaluate for systemic disease, which may identify incidental perineural metastasis. It is important for practitioners to be aware of the potential for neural involvement. Targeted fascicular biopsy may be indicated for cases with progressive neurological deficit and unclear diagnosis.

493 Visceral Fat Volume from Standard Preoperative CT is an Independent Predictor of Survival in Patients Undergoing Surgery for Metastatic Spine Disease

Zachary Pennington; A. Karim Ahmed; Nancy A AbuBonsrah MD; Eric W. Sankey MD; Bart Pielkenrood; C. Rory Goodwin MD, PhD; J.J. Verlaan; Daniel M. Sciubba, MD

Introduction: Life expectancy is a strong determinant of surgical candidacy in patients with spinal metastases. In this study, we aimed to demonstrate whether pre-operative body fat and 
muscle volumes are independent predictors of survival for patients operated on for spinal metastases.

Methods: We retrospectively reviewed patients surgically treated for spinal metastases between 8/1/2004 7/1/2013. Patients who had standard pre-operative abdominal CT scans, acquired within 3 months of surgery, were included in this study. Preoperative and post-operative treatment, pre-operative neurological status and body composition, Charlson Comorbidity Index, primary tumor type, extent of progression, and Tomita and Tokuhashi scores were collected. Multivariable logistic regressions were used to determine independent predictors of $3 \mathrm{mo}, 6 \mathrm{mo}$, and $12 \mathrm{mo}$ survival and the models were assessed with receiver-operating characteristic (ROC) curves. An ROC value greater than 0.7 was considered sufficiently accurate.

Results: We examined 99 patients. The most common primary lesions were lung (16.2\%) and prostate (14.1\%). Independent predictors of 3 mo survival were visceral fat area (VFA) (95\% CI: $1.00-1.18$ per $1000 \mathrm{~mm} 2 ; \mathrm{p}=0.04)$, post-operative chemotherapy $([1.01-3.41] ; \mathrm{p}=0.04)$, and a Tomita score < 3 ([1.17-19.93]; $\mathrm{p}=0.03)$. The only independent predictor of $6 \mathrm{mo}$ survival was VFA ([1.01-1.21] per $1000 \mathrm{~mm} 2 ; \mathrm{p}=0.04)$. Independent predictors of $12 \mathrm{mo}$ survival were subcutaneous fat area ([1.00-1.07] per $1000 \mathrm{~mm} 2 ; \mathrm{p}=0.05)$, breast primary tumor type $([1.17-18.81] ; \mathrm{p}=$ $0.03)$, the absence of visceral metastases ([1.25-4.36]; $\mathrm{p}=0.008)$, and being ambulatory pre-operatively $([1.07-4.95] ; p=0.03)$. The ROC curve AUC values for the $3 \mathrm{mo}, 6 \mathrm{mo}$, and $12 \mathrm{mo}$ models were $0.8196,0.7795$, and 0.8027 , respectively.

Conclusion: Visceral fat mass is a positive independent predictor of post-operative survival in patients treated surgically for spinal metastases. The accuracy of current prognostic tools could be potentially improved via incorporation of visceral fat volume as a risk factor.

494 Efficacy of Routine Biopsy at Vertebral Augmentation for Compression Fracture Repair in the Early Detection of Malignancy in Presumed Benign Vertebral Compression Fractures

\section{Avery M. Jackson, MD; Kimberly R Barber, PhD}

Introduction: Retrospective study assesses the utility of routine biopsy at time of vertebral augmentation, as a tool in the early detection of malignancy in presumed benign VCF.

Methods: Retrospective study conducted on a cohort of consecutive patients undergoing vertebral augmentation between January 2009 to December 2013. 410-bed teaching community hospital. Polymethylmethacrylate cement injection used in every procedure. Biopsy routinely performed at every level of VCF. Outcome measures: SF-36, Visual Analog Scale (VAS), Oswestry Disability Index (ODI), analgesic use, and complications recorded pre- and post-operatively up to 3 years.

Results: Three different levels were augmented in 327 procedures ( 256 patients, mean age $=75.2 \mathrm{yrs}$ ). The most common levels augmented were L1 (64.5\%) and L2 (31.2\%). Analysis of 271 routine biopsies in 256 patients revealed 25 (9.2\%) abnormal biopsy findings. There was an overall cancer diagnosis rate of $1.1 \%$ (3 of 256) when combining patients with no prior history of cancer or cancer thought to be in remission. In these 3 patients, history, examination, laboratory tests, and pre-procedure imaging all failed to suggest malignancy. There were $92(28.4 \%)$ patients with fractures of adjacent vertebra ( $13.0 \%$ inferior, $15.4 \%$ superior). VAS and ODI scores significantly improved; was evident at week 12 and was sustained at up to 1 year post-operatively $(\mathrm{p}<0.01)$. This was not dependent on the levels treated ( 1 vs $>1$ level, $p>0.05$ ), or etiology of VCF ( $\mathrm{p}>0.05) .12$ patients $(4.7 \%)$ had persistent pain not associated with the treated VCF. Complication rate was $1.3 \%$
( 4 of 304). There were 37 deaths, none of which were related to surgery.

Conclusion: Routine biopsy performed during vertebral augmentation does not demonstrate cancer-related VCF's in patients with no previous cancer diagnosis or active malignancy. There was significant improvement in disability at 12 weeks and one year. Adjacent level fractures more likely to be superior to the treated level. 12 patients with persistent pain may be due to other symptomatic osteoporotic levels.

495 Clinical Presentation and Natural History of Intramedullary Spinal Cord Cavernous Malformations

Anshit Goyal, MBBS; Lorenzo Rinaldo, MD, PhD; Redab Alkhataybeh; Kelly D. Flemming, M.D.; Lindsy N. Williams, M.D.; Panagiotis Kerezoudis; Sandy Goncalves, MSc; Mohammed Ali Alvi, MD; Jenna Meyer; Fredric B. Meyer, MD; Mohamad Bydon, MD

Introduction: In comparison to cerebral cavernous malformations, there is a paucity of literature investigating the clinical course of patients with spinal intramedullary cavernous malformations (SCMs). While surgical resection of SCMs is generally recommended after multiple symptomatic hemorrhages, surgical indications are not well-defined due to a limited understanding of the natural history of these lesions. Herein, we present a large, single-institution retrospective case series of SCMs.

Methods: We reviewed records of patients diagnosed with SCMs between 1995-2016. Patients were considered to have a SCM on the basis of radiographic criteria/pathologic confirmation after surgical resection. Hemorrhage was defined as clinical worsening in tandem with radiographic changes visualized on follow-up MRI. Neurologic status was assessed by the Modified McCormick score. Risk factors for hemorrhage were determined using a Cox proportional hazards model.

Results: There were 107 patients meeting our inclusion criteria. The mean age of our cohort was $49.6(+/-17.3)$ years with 48 females $(45.0 \%)$. Follow-up data was available for 85 patients. While $21(24.7 \%)$ patients underwent immediate surgical resection, $64(75.3 \%)$ were initially managed conservatively. Among this group, $16(25 \%)$ suffered a hemorrhage during follow-up and $11(17.2 \%)$ required surgical resection due to interval bleeding or neurologic worsening. The overall annual risk of hemorrhage was $6.2 \%$ per person year. The rate among patients who were symptomatic and asymptomatic on presentation was $9.5 \%$ and $0.8 \%$, respectively. Median time to hemorrhage was 2.3 years (0.1-12.3). Univariate analysis identified increasing SCM size $(p=0.028)$, history of prior hemorrhage $(p=0.013)$ and presence of symptoms $(\mathrm{p}=0.002)$ as risk factors for subsequent hemorrhage. Multivariable proportional hazards analysis revealed large SCM size (Unit RR 1.22, 1.01-1.49; p=0.042) and presence of symptoms to be independently associated with hemorrhage during follow up (RR 9.87, 1.96-179.64, p=0.003)

Conclusion: Surgery may be considered in large, symptomatic SCMs to prevent rebleeding and prevent neurologic worsening.

496 Intramedullary Spinal Cord Metastases: A Single Institutional Review of Survival and Outcomes

Anshit Goyal, MBBS; Yagiz Ugur Yolcu; Panagiotis Kerezoudis; Mohammed Ali Alvi, M.D.; Sandy Goncalves, MSc; Mohamed Elminawy, MBBCh; Mohammed Adeeb Sebai; Mohamad Bydon, MD

Introduction: Intramedullary metastases are rare lesions affecting the spinal cord in patients with disseminated malignancy. 
However, due to increased survival, these lesions are being more frequently diagnosed. Herein, we describe the largest retrospective, single institutional case series on intramedullary spinal cord metastases reported to date in the English-language literature.

Methods: We retrospectively reviewed the clinical course of patients diagnosed with intramedullary metastases at our institution between 1997 and 2016. Patients were considered to have an intramedullary metastasis based on radiographic criteria or pathologic confirmation after surgical resection. Neurologic status was assessed by the Modified McCormick score (MMCS). We analyzed the different approaches to management and factors influencing survival.

Results: We included a total of 67 patients in the final analysis with a total of 85 lesions. Most lesions were found in the thoracic cord $(47 \%)$ followed by cervical $(34 \%)$ and lumbar (18.8\%). Mean age at diagnosis was $59.2 \pm 10.6$ years with $60 \%(n=41)$ being females. Median survival was 107 days (range $1-888$ days). $38 \%$ patients $(n=26)$ received conservative management, 53\% $(n=36)$ received palliative radiotherapy and only $8.8 \%(n=6)$ of patients underwent surgical resection. Age, sex, presence of concomitant brain and other systemic metastasis did not influence survival. However, there was a significant difference in survival in patients with single (median survival -109 days) vs multiple (median survival- 66 days) intramedullary lesions $(\log$ rank $\mathrm{p}=0.0274)$. Although very few patients(n=6) underwent surgical resection, they were found to have better median survival than their conservatively/palliatively treated counterparts(14 months vs 6.6 months, $\mathrm{p}=0.02)$.

Conclusion: The overall survival in patients with intramedullary metastasis remains poor. Surgical management may contribute to improved survival and neurologic outcomes in selected patients. Systemic metastatic burden may not play an important role in survival.

497 Complications Associated with Surgery for Spinal Metastases: A Multivariable Analysis

Jay I. Kumar, BS; Vijay Yanamadala, MD, MBA; Ganesh Shankar, MD, PhD; Bryan D. Choi, MD; John H. Shin, MD

Introduction: Metastases to the spine occur from a variety of primary malignancies. Surgery on these patients can be challenging with a substantial risk of complications. We present a singlecenter experience of 189 consecutive patients who underwent surgery for spinal metastases, and share our insights regarding complications.

Methods: Charts of 189 patients who underwent surgery for spinal metastases over 5 years from October 2011 through February 2017 were reviewed for complications and possible contributing factors. Multivariate logistic regression was performed for patient demographic and surgical parameters that predict complications.

Results: Complications were identified in $20 \%$ of all patients who underwent surgery for spine metastases. Medical complications included: urinary tract infection, $10 \%$; deep vein thrombosis/ pulmonary embolism, 5\%; pneumonia, $3 \%$ and myocardial infarction, $1 \%$. Surgical complications included: wound infection, $3 \%$; and new neurologic deficit, $2 \%$. Average thirty-day survival was $87 \%$. Average ninety-day survival was $65 \%$.

Conclusion: Surgery for spinal metastases is associated with a relatively high complication rate. Medical complications are more common than surgical complications. Optimization of co-morbid conditions by a multidisciplinary team may help reduce medical complications associated with surgery for spinal metastases.

\section{Spinal Astrocytoma: Descriptive Epidemiology from the Central Brain Tumor Registry of the United States}

Jaes C Jones, BS; Samantha Colby; Benjamin Kring; Alex M Witek, MD; Ghaith Habboub, MD; Paramita Das, MS, MD; Min Lang; Josephine Volovetz; Shahed Tish; Quinn T. Ostrom; Carol Kruchko; Jill Barnholtz-Sloan; Varun Ranjan Kshettry, MD; Pablo F. Recinos, MD

Introduction: Spinal astrocytoma is a rare tumor whose epidemiology has not been well defined. We utilized the Central Brain Tumor Registry of the United States (CBTRUS) to provide up-to-date epidemiological data for this disease.

Methods: CBTRUS was queried for astrocytoma and spinal cord based on ICD-O-3 histological and topographical codes, respectively. Age-adjusted incidence per 100,000 persons was calculated and stratified by race, sex, age, and ethnicity. Joinpoint was used to calculate annual percentage change (APC) in incidence.

Results: 2296 spinal astrocytomas were diagnosed in the United States between 1995 and 2014, resulting in an average of approximately 115 spinal astrocytomas annually., The overall age-adjusted incidence was 0.040 (95\% CI, 0.0390 .042$)$, and there was a statistically significant decrease from 0.045 in 1995 , to 0.037 in 2014, which represented an APC of $-1.0 \%$ (95\% CI, $-1.6-0.3$ ) (Figure 1). The peak incidence of 0.057 (95\% CI, 0.053 0.061 ) was found in the under 20 years age group (Figure 2 ). The incidence in males was 0.045 (95\% CI, 0.043-0.048), which was significantly greater than the incidence in females 0.035 (95\% CI, $0.033-0.038)$. No variation by race/ethnicity was seen.

Conclusion: The overall age-adjusted incidence of spinal astrocytoma from 1995 to 2014 was 0.040 per 100,000 , and the rate was trending downward over this period. Perhaps this trend is due to increased accuracy of pathological diagnosis over the study period. The incidence peaks early in life, reaches a nadir in the 20s, and then slowly increases throughout adulthood. The incidence is higher in males than females. This study presents the most comprehensive epidemiology of spinal astrocytoma in the United States to date.

499 Utility of Fenestrated, Cement Augmented, Pedicle Screws for Stabilization of Cancer Related Spinal Instability.

Ori Barzilai, MD; Lily McLaughlin; Eric Lis; Mark H. Bilsky, MD; Ilya Laufer, MD

Introduction: Bone quality in cancer patients is typically poor due to the osteolytic metastases, chemotherapy, radiation and other co-morbidities. Cement augmentation of instrumentation improves osseous purchase of instrumentation in osteoporotic bone and fenestrated screws recently gained FDA approval. . The object of this study was to evaluate the safety and efficacy of cement augmentation via fenestrated screws.

Methods: A retrospective chart review between April 2016 and August 2017. Patients with cancer related spinal instability who underwent spinal stabilization with cement augmentation using fenestrated pedicle screws and were followed up at least 6 weeks post operatively at time of data collection were included. Patient data including demographic, pathologic and treatment variables were collected. Intra operative and post-operative complications were evaluated.

Results: Fifty six patients were included (30 male, 53\%). Cement augmentation was performed in 228 fenestrated pedicle screws. Forty operations $(71 \%)$ included percutaneous stabilization without decompression while 16 (39\%) entailed a central of foraminal decompression. Two patients required re-operation; one 
for revision of instrumentation secondary to a fracture of an instrumented pedicle and one for wound revision. One patient had asymptomatic radiologic proof of a broken pedicle screw 6 months after surgery. No cases of screw pull out, lucency or rod fractures were found. There were no cases of cement extravasation into the spinal canal, yet suspected extravasation into blood vessels was documented in 20 cases and radiologically apparent in 7 . One patient had a-symptomatic evidence of cement in the pulmonary artery, that did not require treatment and without long-term sequelae. One case of in-house mortality was documented secondary to pulmonary failure from widespread metastatic infiltration of the lungs.

Conclusion: Cement augmentation of pedicle screws is an effective method of significantly enhancing durability of spinal constructs in the cancer population. We experienced only one case of pulmonary cement embolization without long-term sequelae and no cases of neurologic deficits.

500 Nine Commonly Cited Scoring Systems in Spinal Oncology: Which is Most Accurate for Your Patient?

A. Karim Ahmed; C. Rory Goodwin, MD, PhD; Amir Heravi; Rachel C. Kim, BS; Nancy A Abu-Bonsrah, MD; Eric W. Sankey, MD; Daniel Kerekes; Camilo A. Molina, MD; Rafael De la GarzaRamos, BA; Joseph H. Schwab; Daniel M. Sciubba, MD

Introduction: Despite advances in spinal oncology, research into patient-based prognostic calculators for metastatic spine disease is lacking. The aim of the current study was to compare the ability of widespread scoring systems to estimate both overall survival at various time points and tumor-specific survival for patients undergoing surgical treatment for metastatic spine disease in order to provide surgeons with information to determine the most appropriate scoring system for a specific patient and timeline.

Methods: A pre-operative score for all 176 patients was calculated utilizing the SORG Classic Scoring algorithm, SORG Nomogram, Original Tokuhashi, Revised Tokuhashi, Tomita, Original Bauer, Modified Bauer, Katagiri, and van der Linden scoring systems. Univariate and multivariate cox proportional hazard models were constructed to assess the association of patient variables with survival. Receiver operating characteristic analysis (ROC) modeling was utilized to quantify the accuracy of each test at different end-points and for different primary tumor subgroups. No funds were received in support of this work. The authors have no conflicts of interest to disclose.

Results: The SORG Nomogram demonstrated the highest accuracy at predicting 30-day (AUC 0.81), and 90-day (AUC 0.70) survival following surgery. The Original Tokuhashi was the most accurate at predicting 365-day survival (AUC 0.78).

Conclusion: Among the nine scoring systems assessed, this study determined the most accurate scoring system for short-term (30-day), intermediate (90-day), and long-term (365-day) survival, relative to primary tumor etiology. This is one of the largest studies comparing the accuracy of multiple scoring systems, the only comprehensive study to include nine scoring systems, and the only study to compare primary tumor-specific prognostic accuracy for this many scoring systems. The findings of this study may be utilized by surgeons in a personalized effort to select the most appropriate scoring system for a given patient.

501 Safety and Efficacy of Fenestrated Pedicle Screws with Cement Augmentation for Spinal Metastases: Early Experience with 202 Screws

Myron Rolle, MD; Bryan D. Choi, MD; Ganesh Shankar, MD, $\mathrm{PhD}$; Laura Van Beaver; Vijay Yanamadala, MD, MBA; John H. Shin, MD
Introduction: Pedicle screw fixation can be a major challenge in patients with spinal metastases.The osteopenic nature of the vertebrae along with factors like previous radiation,immunosuppression, and marrow replacement by cancer affect the bone quality for fixation.Complications related to cement augmentation are known.We share our initial experience with the fenestrated screws.

Methods: IRB approval was obtained and a retrospective analysis was performed on a prospective spine oncology outcomes database.Fenestrated screws became available at our institution in November 2016. We identified 28 consecutive patients who underwent posterior thoracic, lumbar, or sacral decompression and stabilization for pathologic fracture or cord compression(T1-sacrum). Pre-operative SINS (spine instability neoplastic score),ASIA grade,cancer histology,radiation history,ECOG,and patient demographics were recorded and analyzed in a multivariable regression analysis. Post-operative CT was performed in all cases. CT was reviewed and cement extravasation was recorded at each level of pedicle screw placement in 1)the epidural space, 2)neural foramen, 3)paravertebral space, 4)lung, and 5)nearby vasculature including the segmental vessels and aorta.Complications and instrumentation/implant failure were recorded.

Results: Twenty eight patients were identified and a total of 202 fenestrated screws were placed with an mean 7.21 screws implanted per case.The most common construct was 2 levels above and 2 levels below the site of worst vertebral fracture or cord compression.Cement extravasated into the 1)epidural space at 6 levels $(2.97 \%), 2)$ neural foramen at 3 levels $(1.49 \%), 3$ )paravertebral space at 37 levels $(18.3 \%)$, 4)lung - 0,5$)$ nearby vasculature at 15 levels $(7.42 \%)$. There was no cement spread to the aorta or vena cava. No patients developed a symptomatic radiculopathy from foraminal extent. No patient required re-exploration, cement removal, or hardware revision. Follow-up ranged from 1 month to 11 months with no significant change in vertebral body height at the instrumented levels by $\mathrm{CT}$ and xray.

Conclusion: Fenestrated screws with cement provide a safe and effective method for stabilization in the cancer patient.

502 Initial Experience with Intraoperative Phosphorous-32 Brachytherapy During Resection of Malignant Spinal Tumors

Darryl Lau, MD; Stephen T Magill, MD, PhD; Cecilia L. Dalle Ore, B.A.; Igor J. Barani, MD; Vedat Deviren, MD; Manish Kumar Aghi, MD, PhD; Christopher P. Ames, MD

Introduction: Brachytherapy is an adjuvant therapies for neoplastic processes, but few have trialed its use for spinal tumors. This study examines the perioperative and long-term outcomes of malignant spinal tumor patients who underwent resection and intraoperative phosphorous-32 (P32) brachytherapy.

Methods: A consecutive series of adult patients who underwent intraoperative P32 brachytherapy during malignant spinal tumor resection were retrospectively identified from 2014 to 2015 . Complications, perioperative outcomes, tumor recurrence, and survival were reviewed.

Results: A total of 8 patients were included. Average age was 54.3 years and $25.0 \%$ males. Tumor types included: leiomyosarcoma, chordoma, multifocal recurrent ependymomas, breast metastasis, malignant meningioma, and myxofibrosarcoma. Half $(50.0 \%)$ underwent en bloc tumor resection. P32 plaques were applied to 2 sites per patient for a mean of 13.1 minutes with goal penetration of $10 \mathrm{~Gy}$ to $1 \mathrm{~mm}$ depth. Length of hospital stay was 7.1 days. Perioperative complications occurred in 3 of 8 patients $(37.5 \%)$, including wound healing issues in 1 patient who had a persistent cerebral spinal fluid leak following intradural resec- 
tion of a malignant meningioma and 1 patient with an infection $(12.5 \%)$. Ninety-day readmission was $25.0 \%$. Mean length of follow-up was 25.6 months. At most recent follow-up, local recurrence rate was $25.0 \%$, and overall survival was $75.0 \%$. Mean time to recurrence was 14.4 months. Progression free survival (PFS) at 6-, 12-, 18-, and 24-months $100.0 \%, 87.5 \%, 75.0 \%$, and $71.4 \%$, respectively. Survival at 6-, 12-, 18-, and 24-months was $100.0 \%$, $100.0 \%, 85.7 \%$, and $71.4 \%$, respectively.

Conclusion: P32 is safe, practical, and feasible. P32 intraoperative brachytherapy does not increase the risk of wound healing issues or infections compared to external beam radiation. The sample size of this series is small and there is heterogeneity in tumor type, but recurrence and survival outcomes seem promising compared to prior reports. Appropriately empowered clinical trials are needed.

503 Lumbar En Bloc Spondylectomy for Primary Spinal Tumors: A Systematic Review of the Literature

Cecilia L. Dalle Ore, B.A.; Darryl Lau, MD; Christopher P. Ames, MD

Introduction: En bloc spondylectomy (EBS) is an effective but potentially morbid treatment for neoplastic lesions of the spine. Surgical approach and outcomes in lumbar EBS may be distinct from those associated with spinal EBS generally. Objective: To perform an English language systematic literature review of EBS for primary tumors of the lumbar spine, concentrating on surgical approach, complications, local recurrence, and survival.

Methods: The phrase en bloc spondylectomy was used as a search term in PubMed for all years through 2017.

Results: The search identified 196 articles, 63 of which described at least one patient with a primary lumbar lesion. Fortythree articles provided data specifically regarding patients with primary lumbar lesions, describing 113 patients. Of these patients, 73 underwent EBS for malignant lesions and 40 for aggressive benign lesions. The most common lesions were 30 patients with chordoma, 26 with giant cell tumor, 12 with osteosarcoma, 8 with chondrosarcoma, and 6 with Ewing's sarcoma. Surgical approach was available for 79 patients: 31 underwent a combined posterior/anterior ( $\mathrm{p} / \mathrm{a})$ approach, 16 an a/p approach, 13 a posterior-only approach, $10 \mathrm{p} / \mathrm{a} / \mathrm{p}$ approach, and 8 a lateral approach. Complications were available for 68 patients, 35 (51.5\%) of whom experienced at least one complication. Local recurrence data was available for 79 patients, $11(13.9 \%)$ of whom experienced local recurrence. Follow-up was available for 105 patients. At mean 46.3 months follow-up, $13(12.4 \%)$ had died of their disease and 3 $(2.9 \%)$ had died of other causes.

Conclusion: EBS are used in the treatment of primary malignant and aggressive benign lumbar tumors. Combined approaches are frequently used for lumbar EBS. While many patients present with malignant lesions or experience perioperative complications, survival at a mean 46.3 months follow up is approximately $85 \%$.

\section{MIS and Body Habitus}

\section{Matthew R. Quigley, MD}

Introduction: Use of MIS/tubular retraction systems is becoming more widespread, especially as it is perceived to facilitate surgery in the obese. We reviewed our clinical material of MIS lumbar microdiscectomy and decompressions for stenosis to characterize who is a candidate for this approach and whether obesity affects outcome/complications

Methods: A single surgeon/institution practice 1/2015 to 12/2016 using the Medtronic Metrx ${ }^{\circledR}$ system.
Results: There were 110 patients of which 73 were male and the median age was 59. The BMI range was 19.8-48.9 (median 30.2 ), and $75 \%$ were $>25$. The procedures were characterized as following: 53 microdiscectomies, 28 single level decompressions, 15 two level decompressions and 1 three level decompression. All procedures were done through a single $3 \mathrm{~cm}$ transverse incision. Median EBL was $10 \mathrm{cc}$ and length of stay 1 day in $82 / 110$ $(74.5 \%)$ and same day in 22/110 (20\%). There were four incidental durotomies, all treated with fibrin sealant. All of these patients were discharged the next day without CSF leak. Another patient manifested a superficial wound infection responsive to oral antibiotics. Satisfactory outcome (at median follow-up of 19.7 months) was achieved in 93/110 (84.6\%) which did not differ among procedures or by BMI. The only detrimental effect of increasing BMI was greater blood loss $(\mathrm{p}=.02)$. The strongest factor to predict length of tubular retractor required was facet to skin surface measurement on the sagittal MR or CT $(\mathrm{R} 2=0.65, \mathrm{p}=.0001)$, not axial measurement $(\mathrm{R} 2=.56)$ nor $\mathrm{BMI}(\mathrm{R} 2=.39)$. The vast majority of tube length selections were within $1 \mathrm{~cm}$ of the sagittal measurement $(85 \%)$ and all MR measurements up to $10.5 \mathrm{~cm}$ were able to utilize a $9 \mathrm{~cm}$ tube.

Conclusion: MIS procedures can be performed in normal and obese patients with similar outcomes. Given the limits of tube length $(9 \mathrm{~cm})$ procedures can be offered to those with sagittal facet to skin measurements of $=10 \mathrm{~cm}$.

505 The Unreimbursed Costs of Preventing Revision Surgery in Adult Spinal Deformity: Analysis of Cost Effectiveness of Proximal Junctional Failure Prevention Strategies in 195 Patients

Michael Safaee, MD; Cecilia L. Dalle Ore, B.A.; Corinna Clio Zygourakis, MD; Vedat Deviren, MD; Christopher P. Ames, MD

Introduction: Surgical adjuncts for prevention of proximal junctional failure (PJF) have the potential for significant cost savings by preventing readmission and reoperation, yet are often unreimbursed. Hospitals often bear this additional cost despite potential significant benefit to patients.

Methods: Single center, retrospective review of consecutive adult spinal deformity (ASD) patients. PJF was defined as proximal junctional kyphosis requiring revision surgery.

Results: 195 patients were identified with a mean age of 64 years including 135 women and 60 men. An average of 10 levels were fused (range 2-18) with upper instrumented vertebrae (UIV) as follows: 2 cervical, 73 upper thoracic, 108 lower thoracic, and 12 lumbar. PJF prevention strategies included ligament augmentation in 99 cases, hook fixation at the UIV in 60 cases, and vertebroplasty in 71 cases. Average cost of index surgery was $\$ 129,953$. Eighteen patients $(9 \%)$ underwent surgical revision for PJF with a mean follow-up of 22 months. UIV, ligament augmentation, and hook fixation were associated with reduced rates of PJF. In a multivariate model, ligament augmentation had the strongest association with PJF prevention. The average cost of surgical adjuncts was $\$ 1,184$ for ligament augmentation and $\$ 1,176$ for vertebroplasty $(0.9 \%$ cost of index surgery each). Use of hook fixation saves $\$ 560$ per case. The average cost of reoperation for PJF was $\$ 193,277$. Use of ligament augmentation was associated with an absolute risk reduction of PJF of $11 \%$ ( $4 \%$ vs. $15 \%, p=0.011$ ), with a number needed to treat of 9.1 patients.

Conclusion: Ligament augmentation is associated with a significant reduction in revision surgery for PJF in ASD. In a population of 1,000 ASD patients, ligament augmentation adds a total cost of $\$ 1,184,000$ with the potential to prevent 90 revision surgeries at a cost of $\$ 17,394,930$. This dramatic savings warrants consideration for reimbursing surgical adjuncts for PJF prevention. 
506 Cost-effectiveness of a Radiofrequency Hemostatic Sealer to Reduce Need for Transfusion in Adult Spinal Deformity Surgery

Gurpreet Surinder Gandhoke, MD; Kenneth J Smith; Yash Kalpesh Pandya; Nima Alan, MD; David Salvetti, MD; David O. Okonkwo, MD, PhD; Adam S. Kanter, MD

Introduction: The radiofrequency bipolar uses saline-cooled delivery of energy sealing blood vessels instead of burning them. We present a cost-effectiveness analysis when using this device to perform open access adult spinal deformity correction surgery.

Methods: In an observational cohort study, we compared transfusion requirements in 34 patients undergoing surgery for correction of adult spinal deformity utilizing the Aquamantys ${ }^{\circledR}$ hemostatic vessel sealer, (Medtronic, Minneapolis, MN, USA) to that of a control group of 10 patients in which traditional hemostasis was obtained with bipolar electrocautery, and matched them for blood loss related variables. Total expense to the hospital for the index surgery duration of stay was used for cost calculations. The incremental cost effectiveness ratio was calculated using two effectiveness payoffs.

Results: The two groups were similar in age, sex, ASA class, number of levels fused, preoperative hemoglobin, duration of surgery, revision surgery and BMI. The intra-operative blood loss was similar between the 2 groups, (1517 ml Aquamantys ${ }^{\circledR}$ group and $1542 \mathrm{ml}$ in control group, $\mathrm{p}=0.944)$. The peri-operative decrease in hemoglobin between the 2 groups was also similar, (4.06 in the Aquamantys ${ }^{\circledR}$ group and 4.05 in control group, $\mathrm{p}=0.961$ ). The mean total number of blood products transfused in the Aquamantys ${ }^{\circledR}$ group was 5.9 while in the control group it was $4.8, p=0.520$. There was no statistically significant change in the mean Eq5D scores at the 6-week mark between the 2 groups ( 0.0514 vs. 0.0496$), p=0.975$. For both effectiveness payoffs, the cost-effectiveness analysis revealed Aquamantys ${ }^{\circledR}$ to be dominated, costing $\$ 12,360$ more than its non-use, and less effective in terms of quantity of blood products received and quality of life utilities.

Conclusion: The use of the Aquamantys ${ }^{\circledR}$ hemostatic sealer proved to be costlier and did not confer a benefit in reducing intraoperative blood loss or transfusion requirements in patients undergoing open access surgical correction for adult spinal deformity.

507 Diffusion and Cerebrospinal Fluid Flow Magnetic Resonance Imaging in the Evaluation of Cervical Stenosis and Myelopathy: A Prospective Study

Darryl Lau, MD; Cynthia Chin, MD; Philip R. Weinstein, MD; John Engstrom, MD; Christopher P. Ames, MD

Introduction: Diffusion-weighted imaging (DWI) may be more sensitive in detecting early cervical cord injury than abnormal T2-signal. Cerebrospinal fluid (CSF) flow studies may demonstrate degree of stenosis based on cervical cord motion. This study evaluates tests correlation of DWI and cord motion to myelopathy severity and degree of stenosis.

Methods: Prospectively, adult patients with concern for cervical stenosis underwent cervical magnetic resonance imaging (MRI) (T2, Cine CSF Flow, and DWI). Images were reviewed neuroradiologists blinded to the patient's clinical condition. Correlation of MRI findings to neurological status and outcomes following surgery were evaluated.

Results: Twenty patients were enrolled. Mean age was 66 years, and $40 \%$ were male. Eleven presented with myelopathy and 9 had pain, weakness, and/or sensory changes. All patients had radiographic cervical stenosis (14 severe, 5 moderate, and 1 mild). In the presence of cervical stenosis, paradoxical cord motion (moving opposite to the rest of cord) was observed in $79 \%$ of severe and $50 \%$ of mild/moderate stenosis. The sensitivities of MRI findings to detect clinical myelopathy were $63 \%$ for $\mathrm{T} 2$ signal, $73 \%$ for abnormal DWI, 73\% for abnormal cord motion, $91 \%$ for abnormal DWI/cord motion, and $100 \%$ for abnormal T2/DWI/cord motion. Fourteen patients underwent surgical decompression (4 anterior and 10 posterior). Ten of those 14 patients had improved Nurick myelopathy score. Of the 4 patients who did not improve, their MRI profile revealed that all preoperative MRI had abnormal cord motion ( 2 with normal T2-signal and DWI) $(\mathrm{p}=0.126)$. Among the 6 patients ( 1 moderate and 5 severe stenosis) who did not undergo surgery, 1 worsened (abnormal T2-signal/DWI/cord motion) while 5 others had no change in neurological status.

Conclusion: DWI and CSF flow studies are sensitive modalities in detecting myelopathy and evaluating cervical stenosis severity. Abnormal DWI and cord motion are present in myelopathic patients without T2-signal abnormality and maybe useful prognostic indicators.

508 Best Versus Worst Neurological Outcomes after Surgery for Degenerative Cervical Myelopathy: An Ambispective Analysis of A Global AOSpine Cohort of Patients

Rafael De la Garza Ramos, MD; Aria Nouri, MD, MSc; Jonathan P Nakhla, MD; Smruti K. Patel, MD; Merritt Drew Kinon, MD; Joseph S. Cheng, MD, MS; Reza Yassari, MD; Michael G. Fehlings, MD, PhD, FRCS(C), FACS

Introduction: Multiple studies have proven the benefits of surgical intervention for degenerative cervical myelopathy (DCM). Nevertheless, surgical outcomes vary, and there is a subset of patients that unfortunately experience limited benefit from surgery. The purpose of this study is to compare patients with best versus worst functional outcomes after surgery for DCM.

Methods: This is a retrospective review of two prospective multicenter studies (the AOSpine CSM-North America and CSM-International studies) conducted between 2005-2011. Adult patients with DCM who underwent surgical intervention were included. For patients with 2-year follow-up, characteristics were compared between those with best versus worst outcomes based on the modified Japanese Orthopedic Association (mJOA) scale. Only patients with baseline $\mathrm{mJOA}=14$ were included to minimize ceiling effects. Based on the top and bottom $20 \%$ scores, best and worst outcomes were defined as mJOA 18 and $=13$, respectively.

Results: A total of 380 patients with complete mJOA data at 24 months were identified and selected for this study. Out of these 380 patients, 254 had baseline mJOA score of $=14$, corresponding to moderate or severe myelopathy. Based on "best" and "worst" definitions for outcome at 2 years, 107 and 80 patients were respectively identified. On univariate analysis, patients with worse outcomes were older $(\mathrm{p}<0.001)$, more likely to have diabetes $(\mathrm{p}=0.055)$, ossification of the ligamentum flavum $(\mathrm{p}<0.001)$, spondylolisthesis $(\mathrm{p}=0.007)$, circumferential compression $(\mathrm{p}=0.010)$, longer duration of symptoms $(\mathrm{p}=0.023), \mathrm{T} 2$-signal change $(\mathrm{p}=0.004)$, and worse baseline mJOA scores $(\mathrm{p}<0.001)$. On multiple logistic regression analysis, diabetes mellitus $(\mathrm{p}=0.041)$, spondylolisthesis $(\mathrm{p}=0.026)$, duration of symptoms $(\mathrm{p}=0.022)$, T2-signal change $(\mathrm{p}=0.039)$, and baseline mJOA $(\mathrm{p}=0.019)$ were all independent significant predictors of outcome.

Conclusion: In this study, several baseline patient factors were found to significantly differ between those who experienced the best versus worst functional outcome after surgery for DCM. These findings may provide useful for patient counseling, perioperative expectations, and surgical decision-making. 
509 Quantitative MRI of the Cervical Spinal Cord ( to Measure Microstructure and Tissue Injury

\section{Muhammad Akbar, MD; Allan R. Martin, BASc, MD}

Introduction: Conventional MRI imaging only provides limited information about the structure and integrity of the spinal cord. We describe a mutliparametric quantitative MRI protocol for microstructure analysis of the spinal cord to determine the precise degree of injury to the spine in the setting of degenerative cervical myelopathy (DCM) as well as traumatic spinal cord injury.

Methods: 40 healthy controls and 58 DCM patients have so far been studied. Each patient underwent a battery of clinical assessments including mJOA, ISNCSCI, QuickDASH, GRASSP-M and GaitRITE followed by MRI acquisitions using our protocol in a 3T GE clinical scanner. The multi-parametric protocol combines MRI techniques including conventional MRI, Diffusion tensor imaging (DTI), fractional anisotropy (FA), Magnetization transfer (MTR), T2*WI. Image analysis is done using the Spinal Cord Toolbox (SCT) v.3.0.

Results: Study of healthy subjects identifies an alarming rate of asympomatic spinal cord compression. 10 measures of tissue injury were identified that provides diagnosis (AUC=95.4\%) and correlates with disability (mJOA) in a linear regression model ( $\mathrm{R} 2=0.55$ ) Longitudinal study (ongoing) of DCM patients correlates qMRI findings with clinical assessment and suggest mJOA underestimates progression.

Conclusion: Established a reliable, clinically feasible qMRI protocol that can be used for diagnosis, detection of subclinical tissue injury and can be potentially be use for prediction of outcomes in DCM.

510 Are we Altering the Cervical Spine Biomechanics for the Better or Worse?: A Biomechanical Study.

Hoon Choi, MD, MS; Jobin D. John; Narayan Yoganandan, $\mathrm{PhD}$; Mike Arun, PhD; Jamie Baisden, MD

Introduction: Anterior cervical arthrodesis and arthroplasty are the most commonly performed operations in the cervical spine. Disc height and lordosis are the most commonly surgically modified morphometric parameters. This study aims to examine seven major morphometric parameters independently for their effects on the spine biomechanics.

Methods: An experimentally validated parametric finite element model of the entire cervical spinal column was used to examine the biomechanics of the C5-C6 osteoligamentous spinal segment. Seven morphometric parameters were selected for testing: vertebral body size, spinous process-lamina complex size, facet parameters (height, length and slope), disc height, and cervical lordosis. A Latin Hypercube sampling method was used to generate models with randomized variations of the parameters. Four output responses were analyzed for each morphometric variation: range of motion (ROM), anterior longitudinal ligament (ALL) stretch, anterior capsular ligament stretch, and facet compression/ distraction.

Results: Fifty three-dimensional finite element models were generated and complex loading was simulated. Lordosis was the most significant morphometric parameter, resulting in reduced ALL stretch, anterior facet stretch and posterior facet compression, in response to complex loading. Disc height increase had a secondary effect on increasing the range of motion. Resultant ROM in models varied by 4 degrees, and was most affected by the changes in the AP diameter of vertebral body-facet complex, followed by disc height. ALL stretch ranged from 10 to $30 \%$, and it was most affected by lordosis, followed by disc height. Anterior facet capsule stretch was influenced by lordosis, facet height and facet angle. Posterior facet compression was influenced by lordosis and facet angle.

Conclusion: Surgeons should be mindful of the impact of changing morphometric parameters on the spine biomechanics. Cervical lordosis and disc height significantly impact the biomechanics. Facet morphology can significantly alter biomechanics.

511 Radiographic Outcome after Cervical Disc Arthroplasty: Effect of Implant Sizing on Heterotopic Ossification

W. H. Andrew Ryu, BSc, MSc, MTM, MD; Michael M.H. Yang, MD, M.Biotech; R. John Hurlbert, MD, PhD, FACS, FRCS(C)

Introduction: Cervical disc arthroplasty(CDA) has been widely utilized for select patients with degenerative disc disease. However, there are ongoing challenges to CDA including longterm durability to prevent adjacent segment disease(ASD), and development of heterotopic ossification(HO). The aim of this study was to examine radiographic characteristics of CDA in relation to the development of $\mathrm{HO}$ and the association between $\mathrm{HO}$ and ASD.

Methods: Retrospective chart review was performed on patients who underwent 1 to 2-level CDA. For each patient, minimum of 1 year follow-up with serial cervical radiography were analyzed. Radiographs immediately after surgery and at follow-up were examined for $\mathrm{HO}(\mathrm{McAfee}$ classification) and $\mathrm{ASD}$ (Hilibrand classification). Other measurements included global Cobb angle, global range-of-motion, adjacent level disc height, index level angular motion, and overhang of endplate in relation to artificial disc.

Results: Thirty-five patients underwent CDA (mean age 47 years; 20M: $15 \mathrm{~F}$ ) with $74 \%$ receiving 1-level procedure. Mean follow-up duration was 3.3 years (range 18 years). Fifteen patients (43\%) had grade 3 or $4 \mathrm{HO}$ and 9 patients $(26 \%)$ had grade 2 or 3 ASD. Comparing the cohort of patients with high versus low-grade $\mathrm{HO}$, there was a significant difference in the length of endplate overhang (Figure 1; $1.31 \mathrm{~mm}+/-1.76$ vs. $2.59 \mathrm{~mm}+/-1.44, \mathrm{t}=3.25$; $\mathrm{p}=002$ ). Both groups of patients maintained their global alignment, global range-of-motion, and angular motion at the index level up to 8 years. There was no significant association between development of $\mathrm{HO}$ and ASD (chi2 $=2.8 ; \mathrm{p}=0.094$ ). Comparing patients with and without ASD, patients with ASD had significant kyphotic changes in their alignment (5degrees) unlike those without ASD (-2degrees; $t=-2.33 ; \mathrm{p}=0.03)$.

Conclusion: Patients with greater than $2 \mathrm{~mm}$ of overhang of endplate in relation to the artificial disc may be at higher risk of developing HO. Relationship between development of $\mathrm{HO}$ and ASD remains unclear. However, change in sagittal alignment may predict radiographic ASD.

512 Analysis and Temporal Evolution of Extubation Parameters for Patients Undergoing Single Stage Circumferential Cervical Spine Surgery

Ricardo B.V. Fontes, MD, PhD; Mena G Kerolus, MD; Ryan Benjamin Kochanski, MD; Rajeev K. Garg, MD; Anthony DelaCruz; Vincent C. Traynelis, MD

Introduction: Airway obstruction after post-operative extubation is a dreaded but uncommon complication in patients undergoing circumferential cervical spine surgery(CCSS). The cuff leak test(CLT)has been utilized to assess air leak around the 
endotracheal tube(ETT) which may reflect airway swelling.Its PPV is very low in the general ICU population and there is concern that it could promote prolonged intubations.In this prospective observational study,we analyze the temporal evolution of CLT and perioperative factors that may influence it.

Methods: Twenty patients undergoing single-stage $\operatorname{CCSS}(17$ anterior-posterior,3 posterior-anterior-posterior)were followed during their hospital course and managed according to our extubation protocol.Patients were maintained intubated overnight following surgery.They were extubated if a CLT $>200 \mathrm{~mL}$ and both ICU and Neurosurgery teams agreed that it was safe.Patients extubated in the first postoperative day (9/20)comprised the Early group, and the remaining patients (11/20)the Delayed group.Patient and operative data were analyzed as a single group and comparing both groups.

Results: The main indication for surgery was cervical deformity. Median number of levels fused was 5 anteriorly(range,1 6) and 6(1 13) posteriorly.Patients were kept intubated for an average of 73.6(range, 26 222)hours and stayed in the ICU for 119.1 (range, 36 360)hours. There were four failed extubations and three patients(15\%)required a tracheostomy.Patient profiles between both groups were very similar across most patient variables but differed significantly regarding height and infraglottic luminal area $(\mathrm{p}<.05)$.All three patients who required tracheostomy had been decannulated by postoperative day 50

Conclusion: This study objectively demonstrates the difficulties in airway management following CCSS and provides useful insight for preoperative planning and counseling.Our results suggest that local anatomic factors influence airway outcome more than operative factors.The study format does not allow for testing of interventions but we suggest that patients with favorable anatomy(taller,larger infraglottic luminal area)may benefit from a less strict airway management protocol,such as a flexible CLT value as long as an audible leak is present.

513 Incidence of Re-operation for Posterior Cervical Fusion: A Large Scale Retrospective Analysis

Royce Woodroffe, MD; Logan Helland, MD; Patrick W. Hitchon, MD

Introduction: Posterior cervical fusions have been increasingly used to treat a variety of spinal pathologies. With this, there has been an increase in re-operations and construct failures. While it is generally accepted that $\mathrm{C} 2$ provides a rostral anchoring point due to the ability to place a more robust screw, and fusion across the cervico-thoracic junction (CTJ) reduces adjacent segment disease, there are currently no guidelines with regards to extent of fusion required for different scenarios.

Methods: This is a retrospective review of 370 patients who underwent posterior cervical fusions over a 12 year period at a single institution. This study looks at the re-operations, from all causes, of posterior cervical fusions at a single institution and compares different fusion techniques, including the failure rate for subaxial fusions with those that include $\mathrm{C} 2$, as well as those that cross the cervico-thoracic junction.

Results: Of the 370 patients reviewed 44 patients $(11.9 \%)$ that required a revision of any kind. 11 patients $(3.0 \%)$ had failure related to ASD and $5(1.4 \%)$ related to hardware failure. There was not a higher revision rate (for any cause) for patients who had a subaxial fusion and compared with those that included $\mathrm{C} 2$. When looking at biomechanical failures only (adjacent segment disease and screw pullout), patients were less likely to require a revision procedure if their fusion crossed the CTJ $(\mathrm{p}=0.038)$. Of patients requiring revision there were a higher percentage of patients with previous ACDF $(\mathrm{p}<0.001)$
Conclusion: This study did not show an increased risk of failure based on the decision to incorporate $\mathrm{C} 2$; however, there was a significantly increased re-operation rate in patients who did not have fusions crossing the CTJ. When indicated, posterior cervical fusion constructs should cross the CTJ, however there is no evidence to support inclusion of $\mathrm{C} 2$ for prevention of biomechanical failures.

514 Risk Factors Associated with Hardware Failure in Patients Undergoing Surgery for Metastatic Spine Disease

A. Karim Ahmed; Rachel Pedreira; Nancy A Abu-Bonsrah, MD; Rafael De la Garza-Ramos, BA; Eric W. Sankey, MD; C. Rory Goodwin, MD, PhD; Ziya L. Gokaslan MD; Justin Sacks, MD; Daniel M. Sciubba, MD

Introduction: Identifying factors that may contribute to hardware failure is an essential component in treating individuals with metastatic spine disease. The aim of this study was to identify preoperative risk factors associated with hardware failure in patients undergoing surgical treatment for metastatic spine disease.

Methods: A retrospective cohort study was conducted to include patients surgically treated for metastatic spine tumors between 2003 and 2013, at a single institution. A univariate analysis was initially performed to identify associated factors.

Results: 3 patients (1.9\%), of the 159 patients included in the study, had failure of the spine instrumentation. 1 patient had metastatic prostate cancer, and 2 had metastatic breast cancer. Patient demographics, co-morbidities, tumor location, and primary tumor etiology were not found to be statistically significant, with respect to hardware failure. Predictive factors included in the multivariate model were other bone metastasis, visceral metastasis, brain metastasis, Modified Rankin scale, previous systemic chemotherapy, previous radiation to the spine, and mean survival. Previous radiation to the spine was the only factor to be significantly associated $(p=0.029)$, present in all three patients with hardware failure.

Conclusion: Hardware failure is a risk for all patients who undergo instrumentation following resection for metastatic spine tumors. This study identified that pre-operative radiation may increase the risk for hardware failure in this population.

515 Kypho-intraoperative Radiation Therapy: A New Treatment Paradigm for Spine Metastasis. A Phase I/II Clinical Trial

Shashank V Gandhi, MD; Ahmad Latefi, DO; Ferney Diaz Molina, MS; Yu Chen; Maged Ghaly, MD

Introduction: Open surgery followed by SRS is standard of care for patients with unstable spine metastasis. Post-radiation vertebral-compression-fractures (VCF) range from 11-39\%. As radiation is palliative due to systemic tumor burden, improvement in pain and quality of life is paramount. Kyphoplasty intraoperative radiation therapy (Kypho-iORT) is a minimally invasive procedure to radiate metastasis from within vertebral bodies, preventing VCF while providing immediate pain relief in potentially unstable spines.

Methods: This is a prospective phase I/II clinical trial to assess safety and efficacy of Kypho-iORT for potentially unstable spinal metastasis in improving pain and functional status and maintaining local control. Pain scores were assessed with the numerical rating pain scale (NRPS), functional status with brief pain inventory (BPI), local control and VCF with CT/MRI. Patients with symptomatic metastasis underwent intraoperative electron-accelerated radiationbfollowed by kyphoplasty. Tumors were limited to vertebral body with SINS scores 7-12. Intraoperative CT was used to 
delineate tumor volume, confirm transpedicular access to tumor, and conform 10Gy from source to tumor periphery.

Results: 13 levels in 9 patients were treated. Mean age 51.3years. Mean SINS score 9.18 \pm 1.99 . No patients experience neurological deterioration. Mean local-progression-freesurvival was 8.3 months. After 3 months, one patient with metastatic colon adenocarcinoma cancer suffered local progression. Mean VCF-free-survival 8.4 months, with asymptomatic VCF in 1 patient at 6 months. NPRS scores decreased from preoperative $(6.55 \pm 2.54)$ to 1 week $(3.00 \pm 2.57 ; \mathrm{p}=0.002)$, 3months $(3.25 \pm 2.87 ; \mathrm{p}=0.007), 6$ months $(2.00 \pm 2.12 ; \mathrm{p}=0.002)$ and trend toward improvement at 9 months $(2.00 \pm 1.73 ; \mathrm{p}=0.083)$. Functional status improved with reduced mean BPI at 3months $(54.90 \pm 9.29$ vs $37.6 \pm 19.98 ; \mathrm{p}=0.037)$, 6months $(32.0 \pm 18.49 ; \mathrm{p}=0.073)$, and 9 months $(25.67 \pm 5.77 ; \mathrm{p}=0.034)$. Narcotic reduced in $5 / 7$ patients and $3 / 7$ were medication free.

Conclusion: Kypho-IORT is a safe option for potentially unstable spinal metastases. Pain and function significantly improve, enhancing quality of life. Local control and reduction in VCF is maintained to 9 months. Long-term follow-up is necessary to further evaluate efficacy.

516 Adequacy of California Health Insurance for Patient Access to Neurosurgeons

Catherine Miller, MD; Luis Manuel Tumialan, MD; Kenneth Blumenfeld; Praveen V. Mummaneni, MD

Introduction: Since inception of the Affordable Care Act (ACA) in 2010, almost $90 \%$ of all Americans have health insurance. To lower prices and make their plans more affordable, many insurance plans have narrowed their provider networks; thus limiting patient access to specialists such as neurosurgeons. We studied access to neurosurgeons in the state of California for health insurance plans offered through the Covered California network.

Methods: Using California's health insurance marketplace, we identified the currently available health insurance plans. Through each company's website, we generated a list of neurosurgeons included in by the plan's network. Physician information was verified through the California Medical Board website. Locations of neurosurgeons were divided into California counties and narrowed to those with greater than 100,000 populations. This was performed with contributions from the AANS/ CNS Washington Committee and the California Association of Neurological Surgeons.

Results: In California, eleven health insurance companies offer ACA marketplace healthcare plans. Two companies offer plans throughout the entire state, with variable coverage provided by the remainder of the insurance plans. There are 409 neurosurgeons per the California Medical Board. Thirty-five counties have a population of greater than 100,000 people with a variable number of available health care plans (range 2-10). Within these counties, there is also variation in the number of available neurosurgeons, ranging from $1 / 34,121$ to $1 / 228,438$. Two counties with a total population of 456,642 that have no registered neurosurgeon.

Conclusion: Continuing to provide affordable health insurance to the public is the major goal of the ACA. However, narrow networks threaten this aim by limiting access to neurosurgeons. Through this brief overview of California's health insurance offered pursuant to the ACA, we found existing network inadequacies related to patient access to neurosurgeons. With further research, other shortcomings will likely be uncovered; thus highlighting the importance of this public policy issue.
517 Is Patient Satisfaction Associated with 2-year Outcome After Cervical Spine Surgery for Myelopathy?

Saman Shabani, BS, MD; Shekar N. Kurpad, MD, PhD; Matthew Budde, PhD; Marjorie C. Wang MD, MPH

Introduction: Patient satisfaction is often used as a metric for quality of care. However, prior research has not shown a clear association between satisfaction and outcome, especially in cervical spondylotic myelopathy (CSM). The purpose of this study was to analyze the association between mJOA and patient satisfaction after cervical spine surgery for myelopathy.

Methods: We prospectively and continuously enrolled patients undergoing elective cervical spine surgery for CSM at a single center from 2010-2015. MJOA was measured before surgery and up to 2-years post-surgery. Patient satisfaction was measured using a 5-point Likert scale, then dichotomized into "extremely" satisfied versus the other categories. We used Student's t-test to study the association between satisfaction and mJOA, and with achievement of the mJOA minimal clinically important difference (MCID), defined as 2 points. Statistical significance was set at $\mathrm{p}=0.05$.

Results: During the study period, 65 patients were enrolled, and 11 were lost to follow-up at 2-years ( $83 \%$ follow-up). The cohort mean age was 54.6 years, $51 \%$ were female, and mean preop mJOA was 13.17 (SD 2.2). The majority of patients $(80 \%)$ had at least a high school education. $21(39 \%)$ of patients underwent anterior cervical fusion; $27(42 \%)$ posterior cervical fusion; $6(0.1 \%)$ combined approaches. 35 (64.9\%) achieved MCID. We found a significant association between change in mJOA and satisfaction $(\mathrm{p}=0.03)$ and between achievement of MCID for mJOA and satisfaction $(\mathrm{p}=0.04)$.

Conclusion: Patient satisfaction was significantly associated with outcome as measured by mJOA and achievement of MCID in mJOA at 2-years after surgery. Further study should address patient expectations and satisfaction since the goal of CSM surgery is often to preserve function or prevent deterioration.

Disclaimer: The Journal of Neurosurgery Publishing Group (JNSPG) acknowledges that the preceding abstracts are published verbatim as submitted and did not go through either the JNSPG's peer-review or editing process. 